Forschungsberichte aus dem Institut für Kolbenmaschinen Karlsruher Institut für Technologie (KIT) Hrsg.: Prof. Dr. sc. techn. Thomas Koch

Claudius Schück

\title{
Untersuchung der innermotorischen Einflussgrößen auf die Partikelemission eines Ottomotors mit Saugrohreinspritzung
}





\title{
Untersuchung der innermotorischen Einflussgrößen auf die Partikelemission eines Ottomotors mit Saugrohreinspritzung
}

\author{
Zur Erlangung des akademischen Grades eines \\ Doktors der Ingenieurwissenschaften (Dr.-Ing.) \\ von der KIT-Fakultät für Maschinenbau des \\ Karlsruher Instituts für Technologie (KIT) \\ angenommene
}

\section{Dissertation}

von

Dipl.-Ing. Claudius Schück aus Pforzheim 
Forschungsberichte aus dem

Institut für Kolbenmaschinen

Karlsruher Institut für Technologie (KIT)

Hrsg.: Prof. Dr. sc. techn. Thomas Koch

Bibliografische Information der Deutschen Nationalbibliothek

Die Deutsche Nationalbibliothek verzeichnet diese Publikation in der Deutschen Nationalbibliografie; detaillierte bibliografische Daten sind im Internet über http://dnb.d-nb.de abrufbar.

(C) Copyright Logos Verlag Berlin GmbH 2021

Alle Rechte vorbehalten.

ISBN 978-3-8325-5258-9

ISSN 1615-2980

\section{(1) (1) $\Theta \Theta$}

Logos Verlag Berlin GmbH

Comeniushof, Gubener Str. 47, 10243 Berlin

Tel.: +4903042851090

Fax: +4903042851092

INTERNET: http://www.logos-verlag.de 


\section{Vorwort des Herausgebers}

Die Komplexität des verbrennungsmotorischen Antriebes ist seit über 100 Jahren Antrieb für kontinuierliche Aktivitäten im Bereich der Grundlagenforschung sowie der anwendungsorientierten Entwicklung. Die Kombination eines instationären, thermodynamischen Prozesses mit einem chemisch reaktiven und hochturbulenten Gemisch, welches in intensiver Wechselwirkung mit einer Mehrphasenströmung steht, stellt den technologisch anspruchsvollsten Anwendungsfall dar. Gleichzeitig ist das Produkt des Verbrennungsmotors aufgrund seiner vielseitigen Einsetzbarkeit und zahlreicher Produktvorteile für sehr viele Anwendungen annähernd konkurrenzlos. Nun steht der Verbrennungsmotor insbesondere aufgrund der Abgasemissionen im Blickpunkt des öffentlichen Interesses. Vor diesem Hintergrund ist eine weitere und kontinuierliche Verbesserung der Produkteigenschaften des Verbrennungsmotors unabdingbar.

Am Institut für Kolbenmaschinen am Karlsruher Institut für Technologie wird deshalb intensiv an der Weiterentwicklung des Verbrennungsmotors geforscht. Übergeordnetes Ziel dieser Forschungsaktivitäten ist die Konzentration auf drei Entwicklungsschwerpunkte. Zum einen ist die weitere Reduzierung der Emissionen des Verbrennungsmotors, die bereits im Verlauf der letzten beiden Dekaden um circa zwei Größenordnungen reduziert werden konnten aufzuführen. Zum zweiten ist die langfristige Umstellung der Kraftstoffe auf eine nachhaltige Basis Ziel der verbrennungsmotorischen Forschungsaktivitäten. Diese Aktivitäten fokussieren gleichzeitig auf eine weitere Wirkungsgradsteigerung des Verbrennungsmotors. Der dritte Entwicklungsschwerpunkt zielt auf eine Systemverbesserung. Motivation ist beispielsweise eine Kostenreduzierung, Systemvereinfachung oder Robustheitssteigerung von technischen Lösungen. Bei den meisten Fragestellungen wird aus dem Dreiklang aus Grundlagenexperiment, Prüfstandversuch und Simulation eine technische Lösung erarbeitet.

Die Arbeit an diesen Entwicklungsschwerpunkten bestimmt die Forschungs- und Entwicklungsaktivitäten des Instituts. Hierbei ist eine gesunde Mischung aus grundlagenorientierter Forschung und anwendungsorientierter Entwicklungsarbeit der Schlüssel für ein erfolgreiches Wirken. In nationalen als auch internationalen Vorhaben sind wir bestrebt, einen wissenschaftlich wertvollen Beitrag zur erfolgreichen Weiterentwicklung des Verbrennungsmotors beizusteuern. Sowohl Industriekooperationen als auch öffentlich geförderte Forschungsaktivitäten sind hierbei die Grundlage guter universitärer Forschung.

Zur Diskussion der erarbeiteten Ergebnisse und Erkenntnisse dient diese Schriftenreihe, in der die Dissertationen des Instituts für Kolbenmaschinen verfasst sind. In 
dieser Sammlung sind somit die wesentlichen Ausarbeitungen des Instituts niedergeschrieben. Natürlich werden darüber hinaus auch Publikationen auf Konferenzen und in Fachzeitschriften veröffentlicht. Präsenz in der Fachwelt erarbeiten wir uns zudem durch die Einreichung von Erfindungsmeldungen und dem damit verknüpften Streben nach Patenten. Diese Aktivitäten sind jedoch erst das Resultat von vorgelagerter und erfolgreicher Grundlagenforschung.

Jeder Doktorand am Institut beschäftigt sich mit Fragestellungen von ausgeprägter gesellschaftlicher Relevanz. Insbesondere Nachhaltigkeit und Umweltschutz als Triebfedern des ingenieurwissenschaftlichen Handelns sind die Motivation unserer Aktivität. Gleichzeitig kann er nach Beendigung seiner Promotion mit einer sehr guten Ausbildung in der Industrie oder Forschungslandschaft wichtige Beiträge leisten.

Dieses Exemplar der Schriftenreihe umfasst experimentelle Untersuchungen zu den Ursachen der Partikelentstehung in Ottomotoren mit Saugrohreinspritzung sowie die Ableitung möglicher Maßnahmen zur Optimierung der Gemischbildung und somit zur Verringerung der Partikelemission.

Karlsruhe im Februar 2021

Prof. Dr. sc.-techn. Thomas Koch 


\section{Vorwort des Autors}

Die vorliegende Arbeit entstand während meiner Tätigkeit als Doktorand bei der Bosch Engineering GmbH (BEG) in Abstatt in enger, fachlicher Kooperation mit dem Bereich Powertrain Solutions (PS) der Robert Bosch GmbH in Schwieberdingen.

Mein besonderer Dank gilt an erster Stelle Herrn Prof. Dr. sc. techn. Thomas Koch für das mir entgegengebrachte Vertrauen und die mir gebotene Freiheit bei der Gestaltung und Durchführung dieser Arbeit. Die vielen, ausführlichen Fachgespräche, sowie die Ermunterung komplexen Sachverhalten auf den Grund zu gehen, haben mir sehr geholfen und waren eine wertvolle Motivation in den vergangenen Jahren. Für die Übernahme des Korreferats sowie für das Interesse an meiner Arbeit danke ich Herrn Prof. Dr.-Ing. Michael Bargende.

Für die Übernahme der fachlichen Betreuung seitens der Robert Bosch GmbH möchte ich Dr. Wolfgang Samenfink ganz herzlich danken. Die unzähligen Gespräche im Themenfeld der Gemischbildung, deine freundliche und behutsame Art mich stets auf den richtigen Weg zu leiten und deine Geduld mit mir weiß ich wirklich sehr zu schätzen. Besonderer Dank gebührt auch Herrn Dr. Andreas Kufferath. Dank seines Weitblicks und seines Geschicks kam die fruchtbare Kooperation zwischen dem Institut für Kolbenmaschinen (IFKM), der BEG sowie den Abteilungen für ottomotorische Brennverfahrensentwicklung (PS/EPG) und dem Produktbereich (PS-PI/ENI) der Robert Bosch GmbH zustande. Für die Ermöglichung meiner Arbeit sowie für die Finanzierung einiger meiner kostspieligen Messkampagnen gilt mein Dank den Herren Dr. Friedrich Wirbeleit, Dr. Stephan Tafel, Nico Kappel sowie Oliver Towae bei der Bosch Engineering GmbH. Für das große fachliche Interesse an meiner Arbeit, für die Finanzierung des Großteils meiner Untersuchungen sowie für die Ermutigung und Unterstützung bei den Veröffentlichungen meiner Arbeit möchte ich Herrn Dr. Erik Schünemann und Daniel Scherrer herzlich danken.

Bei allen Kollegen der Abteilungen BEG/EAV, PS/EPG, PS/ETS, PS-FI/ENI und PS-PI/ENI sowie den Kollegen am IFKM möchte ich mich für die angenehme Zusammenarbeit, den fachlichen Austausch und die hervorragende Arbeitsatmosphäre bedanken. Die namentliche Erwähnung aller, die mich bei meiner Arbeit unterstützt haben, würde den Rahmen dieses Vorwortes übersteigen. Dennoch möchte ich einigen Kollegen, die über längere Zeit wesentlich zum Gelingen dieser Arbeit beigetragen haben, persönlich danken. Ein großer Dank gilt Herrn Nevzat Acat für seinen unermüdlichen Einsatz am Motorprüfstand auch zu unchristlichen Uhrzeiten. Bei meinem Kollegen Thorsten Klinkhammer möchte ich mich für die wertvolle Unterstützung 
bei der Inbetriebnahme verschiedener optischer Messaufbauten sowie für die angenehmen Gespräche bedanken, die mich auch bei technischen Rückschlägen immer wieder motivierten. Meinem Sitznachbarn Arthur Kiefer danke ich für die sorgsame Behandlung unseres gemeinsamen Versuchsträgers, für das sukzessive Perfektionieren unseres Messaufbaus und für die vielen Gespräche im Büro, die den Arbeitstag sowohl fachlich als auch zwischenmenschlich bereicherten. Ein großer Dank gilt auch den Studenten Wang Le, Moritz Schöck, Armin Hoffmann, Miguel Pacavita, Manuel Lang und Daniel Puscher mit denen ich in Form von Abschlussarbeiten oder Praktika zusammenarbeiten durfte und die mit vorbildlicher Motivation und ihrem engagierten Einsatz zum Gelingen dieser Arbeit beigetragen haben.

Ein kaum in Worte zu fassender Dank gilt meinen Eltern Martina und Joachim Schück, für die Ermöglichung meines Studiums, für den Glauben an meinen Weg und für all ihre bedingungslose Unterstützung in jeglichen Lebenslagen. Meiner gesamten Familie, im Besonderen meinen Schwiegereltern, bin ich unendlich dankbar für den Rückhalt, den ich nicht nur in den vergangenen Jahren der Doktorandenzeit erfahren durfte. Mein größter Dank gebührt meinem Sohn Frieder und meiner Frau Vanessa. Ihr musstet unzählige Stunden auf mich verzichten. Du, liebe Vanessa, hast auch in den Endzügen dieser Arbeit nicht die Geduld mit mir verloren und hast mir immer wieder gezeigt, was wirklich wichtig ist. Worte vermögen nicht zu beschreiben, was du mir bedeutest. 


\section{Abstract}

This research work comprises experiments intending to understand the particulate formation mechanisms of spark ignition (SI) engines with port fuel injection (PFI) as well as the analysis of measures to enhance the mixture formation process in order to reduce particulate number emissions $(\mathrm{PN})$. Basic investigations were performed in an evaporation box, which was designed in the shape of a real intake port. This way not only the evaporation characteristics of different sprays but also the spay-wallinteraction could be evaluated. It is known that fuel wall films are a major source for particulate formation. High-speed images were acquired to analyse and minimize wall fuel films within the combustion chamber of a motored transparent engine using a glass cylinder. This way wall fuel films could be visualized and quantified by means of their surface area. A number of different parameters influencing the emission of PN was investigated on a four-cylinder research engine, which is optically accessible through endoscopes in both the combustion chamber as well as the intake manifold.

Three major sources for particulate formation within the combustion chamber of PFI SI engines could be identified. Wall fuel films at the intake valves and the intake valve seats respectively, wall films at the cylinder liner and inhomogeneities within the gas phase. The profound influence of the gas exchange process on the mixture formation could be visualized by means of high-speed imaging during experiments of manifold pressure variations. A back-flow of hot exhaust gas from the combustion chamber into the intake port facilitates the mixture formation process decisively. Since this supporting effect diminishes for raising manifold pressures, high engine loads present a challenge with regard to PN emission. Therefore the analysis and optimization of further parameters to reduce $\mathrm{PN}$, as for example the injection strategy, the charge motion, the spray design or the fuel pressure, were performed at an operating point with higher engine load and moderate boost pressure. 


\section{Kurzfassung}

Die vorliegende Arbeit umfasst experimentelle Untersuchungen zu den Ursachen der Partikelentstehung in Ottomotoren mit Saugrohreinspritzung sowie die Ableitung möglicher Maßnahmen zur Optimierung der Gemischbildung und somit zur Verringerung der Partikelemission. Grundlegende Untersuchungen zur Gemischbildung wurden an einer Verdunstungskammer in der Gestalt eines Einlasskanals durchgeführt. Die Verdunstungsneigung sowie die Spray-Wand-Interaktion verschiedener Sprayauslegungen konnten so bewertet werden. Kraftstoffwandfilme im Brennraum stellen bekanntlich eine wesentliche Ursache für die Partikelentstehung bei ottomotorischen Brennverfahren dar. Zur Analyse und Minimierung dieser Wandfilme wurden Aufnahmen an einem geschleppten Transparentmotor mit Glaszylinder mit Hilfe von High-Speed-Kameras realisiert. Hierdurch konnten Wandfilme innerhalb des Brennraums visualisiert und anhand ihrer Fläche quantifiziert werden. Eine Vielzahl von innermotorischen Einflussparametern auf die Partikelemission wurde schließlich an einem mit Endoskopen sowohl im Brennraum als auch im Saugrohr optisch zugänglichen Vollmotor untersucht.

Drei dominierende Quellen für die Partikelentstehung im Brennraum von Ottomotoren mit Saugrohreinspritzung konnten dabei identifiziert werden. Kraftstoffwandfilme an den Einlassventilen beziehungsweise den Einlassventilsitzen und an der Zylinderlaufbuchse sowie Inhomogenitäten in der Gasphase. Bei der Untersuchung des Einflussparameters Saugrohrdruck konnte mit Hilfe der bildgebenden Verfahren die herausragende Bedeutung des Ladungswechsels für die Gemischbildung verdeutlicht werden. Beim Öffnen der Einlassventile unterstützt eine Rückströmung vom Brennraum in den Einlasskanal die Aufbereitung des Luft-Kraftstoff-Gemisches in wesentlichem Ausmaß. Da diese unterstützende Wirkung der Einlasskanalrückströmung mit steigendem Saugrohrdruck abnimmt, stellen hohe Motorlasten eine große Herausforderung im Zusammenhang mit der Partikelemission dar. Aus diesem Grund wurde die Analyse und Optimierung weiterer Parameter zur Partikelreduktion, wie zum Beispiel der Einspritzstrategie, der Ladungsbewegung, der Spray-Auslegung oder des Kraftstoffdrucks, vorwiegend bei einem Betriebspunkt oberhalb der saugmotorischen Volllast ausgeführt. 


\section{Inhaltsverzeichnis}

Abstract vii

Kurzfassung viii

Inhaltsverzeichnis \

Nomenklatur

1. Einleitung 1

2. Grundlagen und Stand der Technik 4

2.1. Brennverfahren Saugrohreinspritzung (SRE) . . . . . . . . . . 4

2.2. Gemischbildungsmechanismen . . . . . . . . . . . . . 8

2.2.1. Primärzerstäubung / Strahlzerfall . . . . . . . . . . . 8 8

2.2.2. Sekundärzerstäubung / Tropfenzerfall . . . . . . . . . . . . . . 10

2.2.3. Wandfilmspeicher \& Wandfilmdynamik . . . . . . . . . . . . 11

2.2.4. Verdunstung . . . . . . . . . . . . . . . . . 113

2.3. Partikel - Eigenschaften und Bildungsmechanismen . . . . . . . . 18

2.4. Stand der Technik . . . . . . . . . . . . . . . . . . . 21

2.4.1. Analyse der Gemischbildung im Saugrohr . . . . . . . . . . . . . 21

2.4.2. Analyse der Gemischbildung im Brennraum . . . . . . . . . . . 21

2.4.3. Gemischbildungsoptimierung hinsichtlich gasförmiger Schadstoffemissionen $\left(H C, C O, N O_{x}\right) \ldots \ldots \ldots \ldots$

2.4.4. Partikelemissionen bei Saugrohreinspritzung . . . . . . . . . . 24

2.5. Zielsetzung der Arbeit . . . . . . . . . . . . . . . . . . . 27

3. Versuchsträger und Methoden 28

3.1. SRE Verdunstungskammer . . . . . . . . . . . . . . . . . . . . . . 29

3.1.1. Versuchsaufbau SRE Verdunstungskammer . . . . . . . . . . . 29

3.1.2. Vorgehensweise für Verdunstungsmessungen . . . . . . . . . . . 31

3.1.3. Druckverlaufsanalyse zur Bewertung der Verdunstungsneigung von SRE-Sprays . . . . . . . . . . . . . . . 31

3.2. Geschleppter Transparentmotor . . . . . . . . . . . . . . . . . 37

3.2.1. Versuchsaufbau Transparentmotor . . . . . . . . . . . . . 37

3.2.2. Quantitative Analyse von Kraftstoffwandfilmen im Brennraum . 39

3.3. Vollmotor mit endoskopischen Zugängen . . . . . . . . . . . . . . . . 42

3.3.1. Versuchsträger und Optischer Aufbau . . . . . . . . . . . . 42 
3.3.2. Methodik zur Lokalisierung von Partikel-Quellen . . . . . . . . 45

3.3.3. Partikelmesstechnik . . . . . . . . . . . . . . . . . . 46

3.4. Injektor-Varianten . . . . . . . . . . . . . . . . . . . 48

4. Ergebnisse 49

4.1. Grundlegende Untersuchungen der Gemischbildung im Einlasskanal . . 49

4.1.1. Bewertung von SRE-Injektorsprays anhand ihres Verdunstungsverhaltens . . . . . . . . . . . . . . . . 49 49

4.1.2. Analyse der Strömungsverhältnisse am Einlassventil mittels Ladungswechselrechnung . . . . . . . . . . . 553

4.2. Analyse und Optimierung der Gemischbildung im Brennraum . . . . . 57

4.2.1. Motorlast - gekennzeichnet durch den Saugrohrdruck . . . . . . 57

4.2.2. Steuerzeit Einlassnockenwelle . . . . . . . . . . . . . . . . 60

4.2.3. Einspritztiming . . . . . . . . . . . . . . . . 62 62

4.2.4. Motortemperatur und Transientverhalten . . . . . . . . . . 6 67

4.2.5. Ladungsbewegung . . . . . . . . . . . . . . . . . . . . . . . 77

4.2.6. Spraywinkel bei Single-Konfiguration . . . . . . . . . . . 77

4.2.7. Spraywinkel bei Twin-Konfiguration . . . . . . . . . . . . . . . 855

4.2.8. Kraftstoffdruck . . . . . . . . . . . . . . . 92

4.2.9. Potenzial einer SRE-Mehrfacheinspritzung . . . . . . . . . . 94

5. Zusammenfassung 96

A. Anhang 101

A.1. Exkurs - Kalt-Start \& Katalysatorheizen . . . . . . . . . . . . . . . . . 101

A.2. Veröffentlichte Erfindungsmeldung . . . . . . . . . . . . . . . . . . . . 104

A.3. Ergänzendes Informationsmaterial . . . . . . . . . . . . . . . 109

Abbildungsverzeichnis $\quad \mathbf{1 2 5}$

Tabellenverzeichnis $\quad$ 126

Literaturverzeichnis $\quad 127$

Veröffentlichungen 


\section{Nomenklatur}

\section{Physikalische Größen}

\begin{tabular}{|c|c|c|}
\hline Formelzeichen & Einheit & Bezeichnung \\
\hline$\alpha$ & ${ }^{\circ} \mathrm{KW}$ & Kurbelwinkel \\
\hline$\alpha_{50}$ & $\circ$ & $\begin{array}{l}\text { Winkel zwischen den beiden Strahlen eines E-Typ } \\
\text { Injektors }\end{array}$ \\
\hline$\alpha_{80}$ & $\circ$ & $\begin{array}{l}\text { Öffnungswinkel des Spraykegels eines } \\
\text { Einzelstrahl-Injektors (C-Typ, } 80 \% \text { der } \\
\text { Tropfenmasse umfassend) }\end{array}$ \\
\hline$\alpha_{k}$ & - & $\begin{array}{l}\text { dimensionslose Durchströmzahl der } \\
\text { Ladungswechselorgane mit Bezug zur Kolbenfläche }\end{array}$ \\
\hline$\beta_{80}$ & $\circ$ & $\begin{array}{l}\text { Öffnungswinkel der Spraykegel eines } \\
\text { Zweistrahl-Injektors (E-Typ, } 80 \% \text { der Tropfenmasse } \\
\text { umfassend) }\end{array}$ \\
\hline$\chi_{i}$ & - & Druckverhältnisse im Rahmen der DVA \\
\hline$\gamma$ & $\circ$ & Verkippungswinkel zwischen Spray und Injektorachse \\
\hline$\kappa$ & - & Isentropenexponent \\
\hline$\lambda$ & - & Luft-Kraftstoff-Verhältnis \\
\hline$\Psi$ & - & Druckverhältnis bzgl. $p_{\min }$ im Rahmen der DVA \\
\hline$\rho$ & $\mathrm{kg} / \mathrm{m}^{3}$ & Dichte \\
\hline$\sigma$ & $N / m$ & Oberflächenspannung \\
\hline$\mu$ & $P a \cdot s$ & dynamische Viskosität \\
\hline$A_{e f f}$ & $m^{2}$ & effektive Strömungsquerschnittsfläche \\
\hline$A_{t v}$ & bar $\cdot s$ & Flächenintegral der Tropfenverdunstung aus DVA \\
\hline$A_{V K}$ & $m^{2}$ & Oberfläche der Verdunstungskammer \\
\hline$A_{k}$ & $m^{2}$ & Kolbenfläche \\
\hline$c_{v}$ & $J /(k g \cdot K)$ & spezifische Wärmekapazität bei konstantem Volumen \\
\hline$d_{S l}$ & $\mu m$ & Spritzlochdurchmesser \\
\hline$d_{23}$ & $\mu m$ & Sauterdurchmesser (SMD) \\
\hline$d_{P, a e r}$ & $\mu m$ & Aerodynamischer Partikeldurchmesser \\
\hline fst & - & Anreicherungsfaktor im Kaltstart \\
\hline$h_{V}$ & $J / k g$ & Verdampfungsenthalpie \\
\hline$K_{D V A}$ & - & Bewertungszahl anhand Druckverlaufsanalyse \\
\hline$m_{i n j}$ & $m g$ & Einspritzmasse \\
\hline$N$ & $\min ^{-1}$ & Motordrehzahl \\
\hline Oh & - & Ohnesorge-Zahl \\
\hline$p$ & bar & Druck \\
\hline
\end{tabular}




\begin{tabular}{|c|c|c|}
\hline Formelzeichen & Einheit & Bezeichnung \\
\hline$p_{\text {Kammer }}$ & bar & Kammerdruck \\
\hline$p_{\text {sat }}$ & mbar & Sättigungsdampfdruck \\
\hline$p_{0}$ & mbar & Initialer Druck bei $\mathrm{t}=0 \mathrm{~s}$ \\
\hline$q_{d y n}$ & $m g / H u b$ & $\begin{array}{l}\text { dynamischer Massendurchfluss eines Injektors pro } \\
\text { Nadelhub als Funktion der Zeit }\end{array}$ \\
\hline$Q_{\text {stat }}$ & $g / \min$ & stationärer Injektor-Durchfluss \\
\hline$Q_{v}$ & $J / s$ & Verdunstungswärmestrom \\
\hline$Q_{w}$ & $J / s$ & Wandwärmestrom \\
\hline$R$ & $J /(k g \cdot K)$ & spezifische Gaskonstante \\
\hline$R e$ & - & Reynolds-Zahl \\
\hline$T$ & $K$ & Temperatur \\
\hline$t_{\text {Flug }}$ & $m s$ & Flugdauer der Spraytropfen \\
\hline$t_{o f f}$ & $m s$ & $\begin{array}{l}\text { Ventilverzugszeit zwischen elektrischer Ansteuerung } \\
\text { und Nadelschließen }\end{array}$ \\
\hline$t_{o n}$ & $m s$ & $\begin{array}{l}\text { Ventilverzugszeit zwischen elektrischer Ansteuerung } \\
\text { und Nadelöffnen }\end{array}$ \\
\hline$T_{0}$ & ${ }^{\circ} \mathrm{C}$ & Initiale Temperatur bei $\mathrm{t}=0 \mathrm{~s}$ \\
\hline$T_{a}$ & ${ }^{\circ} \mathrm{C}$ & Temperatur außen \\
\hline$t_{e}$ & $m s$ & elektrische Ansteuerdauer eines Injektors \\
\hline$T_{i}$ & ${ }^{\circ} \mathrm{C}$ & Temperatur innen \\
\hline$t_{i}$ & $m s$ & Einspritzdauer (hydraulisch) \\
\hline$U$ & $J$ & Innere Energie \\
\hline$v$ & $\mathrm{~m} / \mathrm{s}$ & Geschwindigkeit \\
\hline$V$ & $m^{3}$ & Volumen \\
\hline$W$ & $J$ & Arbeit \\
\hline$W e$ & - & Weber-Zahl \\
\hline
\end{tabular}




\section{Abkürzungen und Indizes}

\section{Abkürzung \\ Bezeichnung}

Abg.

aer.

AGR

AV

BDE

BMEP

$\mathrm{CF}$

CMF

cVI

DI

DVA

EOI

ES

EV

EV14

EVö

EVs

fl

g

GW

HSVE

IGN

IMEP

Inj.

isfc

$\mathrm{Kr}$

krit.

L

LBK

LW-OT

Mach

MAPi

MLV

NEDC

NTE

NW

oVI

$\mathrm{P}$

P-DI

Auslassventil

Einspritzung

Einlassventil

Flüssigkeit

Gas

Grenzwert consumption)

Kraftstoff

kritisch

Luft

Mehrlochventil

Nockenwelle

Partikel
Abgas
aerodynamisch

Abgasrückführung

Benzindirekteinspritzung

Effektiver Mitteldruck (Break Mean Effective Pressure)

Conformity Factor

Ladungsbewegungsklappe (Charge Motion Flap)

Vorgelagerte Einspritzung (closed Valve Injection)

Benzindirekteinspritzung (Direct Injection)

Druckverlaufsanalyse

Einspritzende (End of Injection)

Einspritzventil vom Typ Bosch EV14

Einlassventil öffnet

Einlassventil schließt

High-Speed Video-Endoskopie

Zündzeitpunkt (Ignition)

indizierter Mitteldruck (Indicated Mean Effective Pressure)

Einspritzung (Injection)

Indizierter spezifischer Kraftstoffverbrauch (indicated specific fuel

Ladungsbewegungsklappe

Oberer Totpunkt im Ladungswechsel

Schallgeschwindigkeit

indizierter Druck im Einlasskanal (indicated Manifold Air Pressure)

New European Drive Cycle

Not to exceed (NTE-Limit $=$ CF x EU6-Limit)

Saugsynchrone Einspritzung (open Valve Injection)

Kombiniertes System aus Saugrohr- und Direkteinspritzung (Port and Direct Injection) 


$\begin{array}{ll}\text { Abkürzung } & \text { Bezeichnung } \\ & \\ \text { PEMS } & \text { Portable Emissions Measurement System } \\ \text { PFI } & \text { Saugrohreinspritzung (Port Fuel Injection) } \\ \text { PM } & \text { Partikelmasse } \\ \text { PN } & \text { Partikelanzahl (Particulate Number) } \\ \text { Rail } & \text { Kraftstoffdruckspeicher (Fuel Rail) } \\ \text { RDE } & \text { Real Driving Emissions } \\ \text { rel } & \text { relativ } \\ \text { ROI } & \text { Region of Interest } \\ \text { Sl } & \text { Spritzloch } \\ \text { SMD } & \text { Mittlerer Sauter-Durchmesser (Sauter Mean Diameter) } \\ \text { SOI } & \text { Einspritzbeginn (Start of Injection) } \\ \text { SR } & \text { Saugrohr } \\ \text { SRE } & \text { Saugrohreinspritzung } \\ \text { Tr } & \text { Tropfen } \\ \text { UV } & \text { Ultra-Violett } \\ \text { WLTC } & \text { Worldwide harmonized Light vehicles Test Cycle } \\ \text { Z-OT } & \text { Oberer Totpunkt nahe dem Zündzeitpunkt } \\ \text { Zyl } & \text { Zylinder }\end{array}$




\section{Einleitung}

Trotz der zunehmenden Marktdurchdringung der Benzindirekteinspritzung (BDE) ist ein Großteil der Ottomotoren in Kraftfahrzeugen aktuell noch mit konventioneller Saugrohreinspritzung (SRE) ausgestattet. Den Recherchen anhand von Verkaufszahlen des ICCT zufolge betrug der Marktanteil der Saugrohreinspritzung unter den Ottomotoren in Europa im Jahr 2018 noch 43\% [78], in China im Jahr 2017 gar $68 \%$ 124. In den USA kommt die EPA für das Jahr 2018 auf einen Marktanteil von $47 \%$ [32]. Die technischen Vorteile der Benzindirekteinspritzung, wie etwa die reduzierte Klopfneigung und somit ein besserer thermodynamischer Wirkungsgrad, oder eine höhere, erzielbare spezifische Leistung, insbesondere in Kombination mit einer Aufladung des Motors, liegen auf der Hand. Dennoch stellt die Saugrohreinspritzung, dank der überschaubaren technischen Komplexität, sowohl hardware- als auch softwareseitig, insbesondere für kostensensible Marktsegmente ein attraktives Konzept dar. Auch in Märkten mit minderer Kraftstoffqualität ist das Brennverfahren SRE aufgrund seiner Robustheit im Vorteil. Es ist zu erwarten, dass im Zusammenhang mit der Hybridisierung der Antriebsstränge, die SRE auch langfristig eine wesentliche Rolle spielen kann [92].

Mit Schlagzeilen wie „Ottomotoren, dreckiger als Diesel“ und „Klein, aber Schwein“ kritisierte die Süddeutsche Zeitung im März 2015 auf ihrer Onlinepräsenz die hohen Rußemissionen des Renault Twingo und des Smart Forfour. Die beiden Fahrzeuge mit baugleichem Dreizylindermotor mit 1.0 l Hubraum waren im ADAC EcoTest aufgefallen, konkret im ADAC Autobahnzyklus („BAB-130“), weil es sich um einen Motor mit Saugrohreinspritzung handelt. Vorwiegend bei Ottomotoren mit Benzindirekteinspritzung galt die Reduktion der Rußemissionen, bzw. der Partikelemissionen, als technisch anspruchsvoll. Diesbezüglich gab es in den vergangen Jahren erhebliche Anstrengungen in Forschung und Entwicklung. Nicht zuletzt weil sich die Gesetzgebung in der EU für die Schadstoffgrenzwerte der Partikelemissionen auf Ottomotoren mit Direkteinspritzung beschränkt. Offenbar bedarf es aber auch für Ottomotoren mit Saugrohreinspritzung Maßnahmen, die die Partikelemission reduzieren. Der aktuell angestrebte Ansatz, auch für Ottomotoren geeignete Partikelfilter zu etablieren, erscheint naheliegend, nachdem beim Dieselmotor die Rußthematik, dank des Dieselpartikelfilters, als technisch gelöst angesehen werden darf. Dennoch gilt es auch beim Einsatz eines Partikelfilters, die Rohemissionen zu minimieren. Sind die Partikelrohemissionen gering, fällt es leichter einen Filter hinsichtlich geringem Abgasgegendruck und somit besserem Motorwirkungsgrad auszulegen. 
Die Gesetzgebung, welche für die Europäische Union die Schadstoffemissionen für Personenkraftwagen mit Verbrennungsmotoren reglementiert, befindet sich zur Zeit im Wandel. Hauptverantwortlich für diesen Wandel ist die Einführung des Messverfahrens für Emissionsmessungen im Straßenverkehr (EU6-RDE) mit einem „portablen Emissionsmesssystem" (PEMS). Zum 01.09.2017 trat die Verordnung 2017/1151 der EU Kommission in Kraft [26] und somit die Emissionsgesetzgebung für die Abgasnorm EU6d-temp, welche am 01.01.2020 von der Abgasnorm EU6d abgelöst wurde. Der weltweit harmonisierte Prüfzyklus für leichte Nutzfahrzeuge (Worldwide harmonized Light vehicles Test Cycle, WLTC) wurde dabei eingeführt und ersetzte das Fahrgeschwindigkeitsprofil des bisher gültigen „Neuen europäischen Fahrzyklus“ (New European Drive Cycle, NEDC). Ein wichtiger Schritt hin zu einem realistischeren Fahrprofil auf dem Rollenprüfstand, mit deutlich weniger Konstantfahrten, kürzeren Standzeiten, höherer Höchstgeschwindigkeit und stärkeren Beschleunigungen. Die Einführung des EU6-RDE-Testverfahrens stellte jedoch die weitaus größere Veränderung dar.

Aus der Perspektive der Gesetzgeber galt es Rahmenbedingungen zu schaffen, welche die Ermittlung von realistischen, fairen und vergleichbaren Emissionsmesswerten ermöglichen. Bei Fahrten im Straßenverkehr mit dem Einfluss des individuellen Fahrstils des einzelnen Testfahrers ist das, im Vergleich zu einer vorgegebenen und gut reproduzierbaren Fahrkurve auf dem Rollenprüfstand, eine Herausforderung. Aus Sicht der Fahrzeughersteller galt es nun sicherzustellen, dass die Schadstoffemissionen unter allen im EU6-RDE-Test möglichen Fahrzuständen die Grenzwerte unterschreiten, also unter Umständen auch bei Volllast. Für die Details zu den Anforderungen an eine gültige EU6-RDE-Fahrt, zu den vorgeschriebenen Auswertungsmethoden und zur portablen Emissionsmesstechnik [5, 49, 50] sei an dieser Stelle auf die Literatur verwiesen [21 24, 26, 27]. Die Quintessenz aus der neuen EU6-RDE-Gesetzgebung für die vorliegende Arbeit liegt in der Tatsache, dass deutlich höhere Motorlasten relevant geworden sind, und dass neben Stickstoffmonoxid und Stickstoffdioxid (NOx) insbesondere die Partikelanzahlemission (Particulate Number, PN) im realen Fahrbetrieb $\mathrm{zu}$ optimieren ist.

Unter der Annahme, dass die, sich gerade etablierende, portable Messtechnik aktuell noch mit einer erhöhten Messunsicherheit behaftet ist, gewährt der Gesetzgeber bei RDE-Messungen die Multiplikation der EU6-Schadstoffgrenzwerte mit einem "Conformity factor $(\mathrm{CF})$ “, welcher langfristig entfallen soll. Die aktuell geltenden Grenzwerte sind in Tabelle 1.1 zusammengestellt. Die Kohlenwasserstoffemissionen (THC) unterliegen derzeit noch keinem NTE Limit („not to exceed“) für RDE-Messungen, wohl aber dem EU6 Grenzwert für die Überprüfung im WLTC. Ähnliches gilt für die Kohlenstoffmonoxidemissionen (CO), mit dem Unterschied, dass das Resultat der RDE-Messung zwar dokumentiert werden muss, jedoch noch nicht durch ein NTELimit reglementiert ist. Im Kontext der vorliegenden Arbeit ist hervorzuheben, dass speziell für Ottomotoren mit Saugrohreinspritzung in Europa aktuell noch kein PN Grenzwert existiert. Der in Tabelle 1.1 aufgeführte Grenzwert gilt explizit nur für Ottomotoren mit Direkteinspritzung. Es zeichnet sich jedoch ab, dass in einer künf- 


\begin{tabular}{l|c|c|c|c} 
& NOx & PN $^{(*)}$ & CO & THC \\
\hline Emissions Limit EU6 & $60 \mathrm{mg} / \mathrm{km}$ & $6 \cdot 10^{11} \# / \mathrm{km}$ & $1000 \mathrm{mg} / \mathrm{km}$ & $100 \mathrm{mg} / \mathrm{km}$ \\
Aktuelle CF & $1+0.43$ & $1+0.5$ & - & - \\
NTE Limit (RDE) & $85,8 \mathrm{mg} / \mathrm{km}$ & $9 \cdot 10^{11} \# / \mathrm{km}$ & - & -
\end{tabular}

Tabelle 1.1.: EU6 Schadstoffemissionsgrenzwerte für Ottomotoren 22] sowie NTE Limits (Not to exceed) für RDE [25-27], wobei NTE Limit = CF x EU6 Limit.

(*): PN Grenzwert gilt nur für Ottomotoren mit Direkteinspritzung

tigen Abgasnorm EU7 diese Unterscheidung in der ottomotorischen Gemischbildung bezüglich des PN-Grenzwerts entfallen wird [34].

Voraussichtlich noch vor der europäischen Union werden in China mit der Abgasnorm China 6a Emissionsgrenzwerte eingeführt, die unabhängig vom Kraftstoff und auch unabhängig von der Art der Einspritztechnik zur Anwendung kommen [77]. Ab dem 01.07.2020 existiert dann weltweit erstmalig, auch für Ottomotoren mit Saugrohreinspritzung, ein PN-Grenzwert von $6 \cdot 10^{11} \# / \mathrm{km}$. Dies unterstreicht die dringende Notwendigkeit die Partikelentstehung bei ottomotorischen Brennverfahren mit Saugrohreinspritzung grundlegend zu analysieren, um Optimierungsmaßnahmen zur Reduktion der PN-Emissionen ableiten zu können.

Zur Partikelentstehung bei Ottomotoren mit Benzindirekteinspritzung gibt es zahlreiche wissenschaftliche Arbeiten. Zu den Mechanismen der Partikelentstehung bei Ottomotoren mit Saugrohreinspritzung hingegen ist noch relativ wenig bekannt. Die vorliegende Arbeit befasst sich daher mit der Untersuchung von innermotorischen Einflussgrößen auf die Partikelemission von Ottomotoren mit Saugrohreinspritzung. Die angestrebten Ziele dieser Arbeit sind, die für die Partikelemission kritischen Betriebsmodi zu ermitteln, die Mechanismen zur Partikelentstehung speziell für SRE zu analysieren und die Einflussparameter auf die Partikelentstehung zu untersuchen, um letztendlich Optimierungsansätze ableiten zu können, insbesondere auch für die Spray-Auslegung des Injektors. 


\section{Grundlagen und Stand der Technik}

\subsection{Brennverfahren Saugrohreinspritzung (SRE)}

Die elektronisch geregelte, intermittierende Saugrohreinspritzung ist seit vielen Jahren Stand der Technik für Ottomotoren mit äußerer Gemischbildung. Historische Vorgänger wie Vergaser oder mechanisch gesteuerte Einspritzsysteme (z.B.: K-Jetronic) werden den heutigen Anforderungen hinsichtlich der Zumessgenauigkeit des Kraftstoffes nicht gerecht. Für eine effektiv arbeitende Abgasnachbehandlung in Form eines DreiWege-Katalysators ist es erforderlich, die jeweils für eine stöchiometrische Verbrennung benötigte Kraftstoffmasse für jeden Arbeitstakt möglichst exakt und zylinderindividuell zu dosieren. Die Komplexität der Gemischbildung für ein Brennverfahren mittels Saugrohreinspritzung wird anhand der sich überlagernden und gegenseitig beeinflussenden Vorgänge deutlich, die im Folgenden näher beschrieben werden. Abbildung 2.1

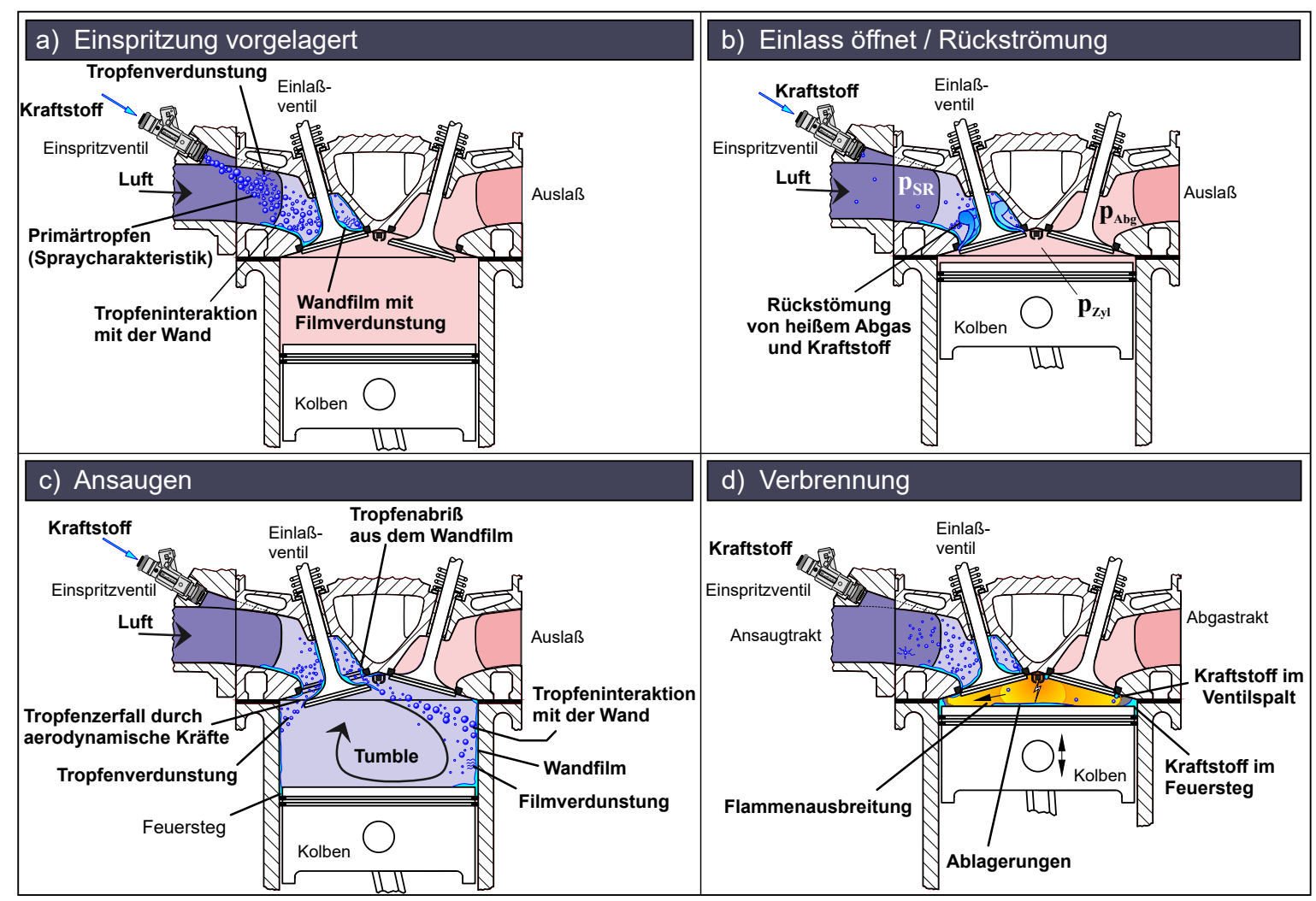

Abbildung 2.1.: Gemischbildungsprozess nach Samenfink [102] 
zeigt die für die Gemischbildung wesentlichen Phasen eines Arbeitszyklus. Im Bildbereich a) ist eine vorgelagerte Einspritzung vor die noch geschlossenen Einlassventile dargestellt. Ausgehend von der Primärzerstäubung (siehe auch Abschnitt 2.2.1) des Kraftstoffes durch das Einspritzventil eröffnen sich mehrere Pfade für den Phasenübergang der flüssigen Kraftstofftröpfchen zu einem gasförmigen und möglichst homogenen Luft-Kraftstoff-Gemisch [62]. Neben der angestrebten Tropfenverdunstung ist auch mit einer Interaktion des Kraftstoffsprays mit den Kanalwänden und dem Einlassventil zu rechnen. Die Eigenschaften der resultierenden Tropfen, nach einer Interaktion mit einer trockenen oder bereits benetzten Wand, wurden detailliert in der Dissertation von Samenfink [101] untersucht. Kraftstoffwandfilme können wiederum ebenfalls verdunsten. Dies ist insbesondere auf der heißen Rückseite der Einlassventile zu erwarten, wie anhand von Temperaturmessungen von Rottenkolber et al. [95] und Alkidas [1] gezeigt werden konnte. Einen entscheidenden Mechanismus der Gemischbildung mit SRE zeigt Abbildung 2.1 b). Für Saugrohrdrücke unterhalb der saugmotorischen Vollast liegt zum Zeitpunkt des Einlassventilöffnens ein negatives Druckgefälle zwischen Brennraum und Einlasskanal vor.

$$
p_{S R}<p_{Z y l} \quad \text { mit } \quad p_{Z y l} \approx p_{A b g}
$$

Dies führt zu einer vorübergehenden Rückströmung von heißem Abgas in den Einlasskanal. Dadurch werden an den Einlassventilen befindliche Kraftstoffwandfilme in den Einlasskanal zurückgedrängt und die Kraftstoffverdunstung erheblich begünstigt. Mit Fortschritt des Ansaugvorgangs kommt die Rückströmung zum Erliegen. Es kommt zur Umkehr in eine Vorwärtsströmung und somit zum Gemischtransport in den Zylinder, wie Abbildung 2.1 c) zeigt. Noch nicht verdunstete Kraftstofftröpfchen gelangen in den Brennraum, wobei einige dort mit Wänden wie Zylinderlaufbahn, Einlassventilsitzen, Kolben und Brennraumdach interagieren und Kraftstoffwandfilme bilden. Im Fall von instabilen, sogenannten überkritischen Filmströmungen, entstehen nach Elsässer [31] neue Kraftstoff-Ligamente oder Tröpfchen durch Herausreißen aus schubspannungsgetriebenen Wandfilmen. In Abhängigkeit von Tropfengröße und Relativgeschwindigkeit zwischen Luft und Tropfen stellt die Sekundärzerstäubung aufgrund von aerodynamischen Kräften einen weiteren Gemischbildungsmechanismus dar, wie Samenfink et al. [104] ermitteln konnten. Dieser Effekt wird aufgrund seiner wesentlichen Bedeutung für diese Arbeit in Abschnitt 2.2.2 ausführlicher beschrieben. Hinsichtlich Wirkungsgrad und Schadstoffemissionen ist es entscheidend, wie viel der flüssigen Kraftstoffanteile in Form von Tröpfchen und Wandfilmen bis zum Zeitpunkt der Entflammung noch vollständig verdunstet werden können (siehe Abbildung 2.1d).

Das Einspritzventil ist in der Regel so angeordnet, dass die Distanz des Abspritzpunktes zu den Einlassventilen möglichst gering ausfällt, um die nahezu unvermeidliche Benetzung des Saugrohrs und der Kanalwände minimal zu halten. Hintergrund dafür ist die unerwünschte Speicherung von Kraftstoff in Wandfilmen im Einlasskanal, welche bei transientem Motorbetrieb zu Abweichungen in der Kraftstoffdosierung führt [37]. Der Kraftstoffdruck beträgt je nach Systemauslegung üblicherweise zwischen drei und 
fünf bar, in Sonderfällen auch bis zu zehn bar [68]. Jedem Zylinder stehen ein oder gar zwei Einspritzventile zur Verfügung („Single“- oder „Twin“-Konfiguration). Mit zwei Einspritzventilen pro Zylinder ergeben sich weitere Freiheitsgrade bei der Sprayauslegung. Neben dem höheren darstellbaren Kraftstoffmassenstrom besteht nach Posselt et al. [86] ein Potenzial für ein optimiertes Tropfengrößenspektrum. In Abhängigkeit der Einbauposition des Einspritzventils („Injektor“) sowie der Einlasskanalgeometrie des Motors ist die Sprayauslegung („Spray-Targeting“) für jeden Motor spezifisch zu gestalten. Die bei Bosch Injektoren vom Typ EV14 zur Verfügung stehenden Strahlformen, sowie die entsprechenden Strahlwinkeldefinitionen sind in Abbildung 2.2b dargestellt. Sprays in der Form eines Kegelstrahls kommen in der Regel

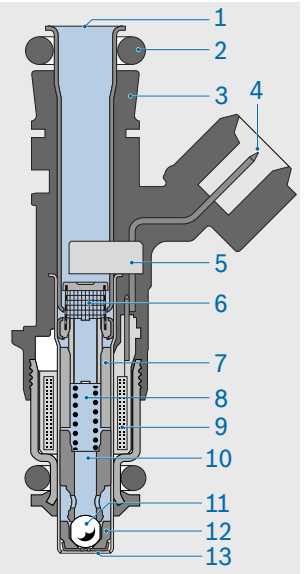

(a) EV14

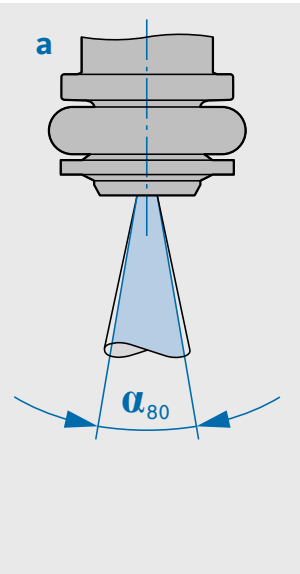

(b) Strahlformen: a Kegelstrahl; b Zweistrahl; c Geneigtes Strahlbild

Abbildung 2.2.: Elektromagnetisches Einspritzventil vom Typ Bosch EV14 sowie Spraywinkeldefinitionen [89]

bei bei Ausrichtung des Injektors auf ein einzelnes Einlassventil zum Einsatz, also bei Twin-Konfigurationen oder bei Motoren mit nur einem Einlassventil pro Zylinder. Am häufigsten ist heutzutage die Strahlform Zweistrahl, wobei jeder Strahl auf die Rückseite eines Einlassventils ausgerichtet wird. Sofern es die Einbauposition des Injektors erfordert, kann das Strahlbild um einen $\gamma$-Winkel verkippt werden. Die für die Gemischbildung wesentlichen Bestandteile des EV14 sind Ventilnadel (10), Ventilkugel (11), Ventilsitz (12) und Spritzlochscheibe (13) (siehe Abbildung 2.2a). Insbesondere das Design der Spritzlochscheibe wurde in der Vergangenheit weiterentwickelt, um ein homogenes Tropfengrößenspektrum mit möglichst kleinen Tröpfchen zu erzielen [45, 103]. Ziele bei der Optimierung von Spritzlochscheibe und Spray-Targeting sind möglichst hohe Verdampfungsraten sowie eine möglichst geringe Wandbenetzung unter Berücksichtigung von Kanalgeometrie und Strömungsverhalten der Ansaugluft. Die physikalischen Mechanismen, welche für die Ausbildung des Primärtropfensprays verantwortlich sind, werden nachfolgend in Abschnitt 2.2.1 ausführlicher beschrieben.

Der große Einfluss des Einspritzzeitpunktes auf die Gemischbildung bei Saugrohreinspritzung - insbesondere im Hinblick auf gasförmige Emissionen und Verbrennungs- 
stabilität - wurde in zahlreichen Arbeiten untersucht. Dazu gehören unter anderen die Dissertation von Lang [59], Werke von McGee et al. [67], Meyer und Heywood [75, 176], Rottenkolber et al. [95] und Schünemann et al. [108]. Das Motorsteuergerät ermöglicht die Vorgabe eines optimalen Einspritzzeitpunktes in Abhängigkeit von Motordrehzahl, Motortemperatur, Saugrohrdruck bzw. Motorlast und Betriebsstrategie. Bei der Saugrohreinspritzung wird zwischen vorgelagerter und saugsynchroner Einspritzung unterschieden (siehe Abbildung 2.3). Bei früher Einspritzung vor die noch geschlossenen

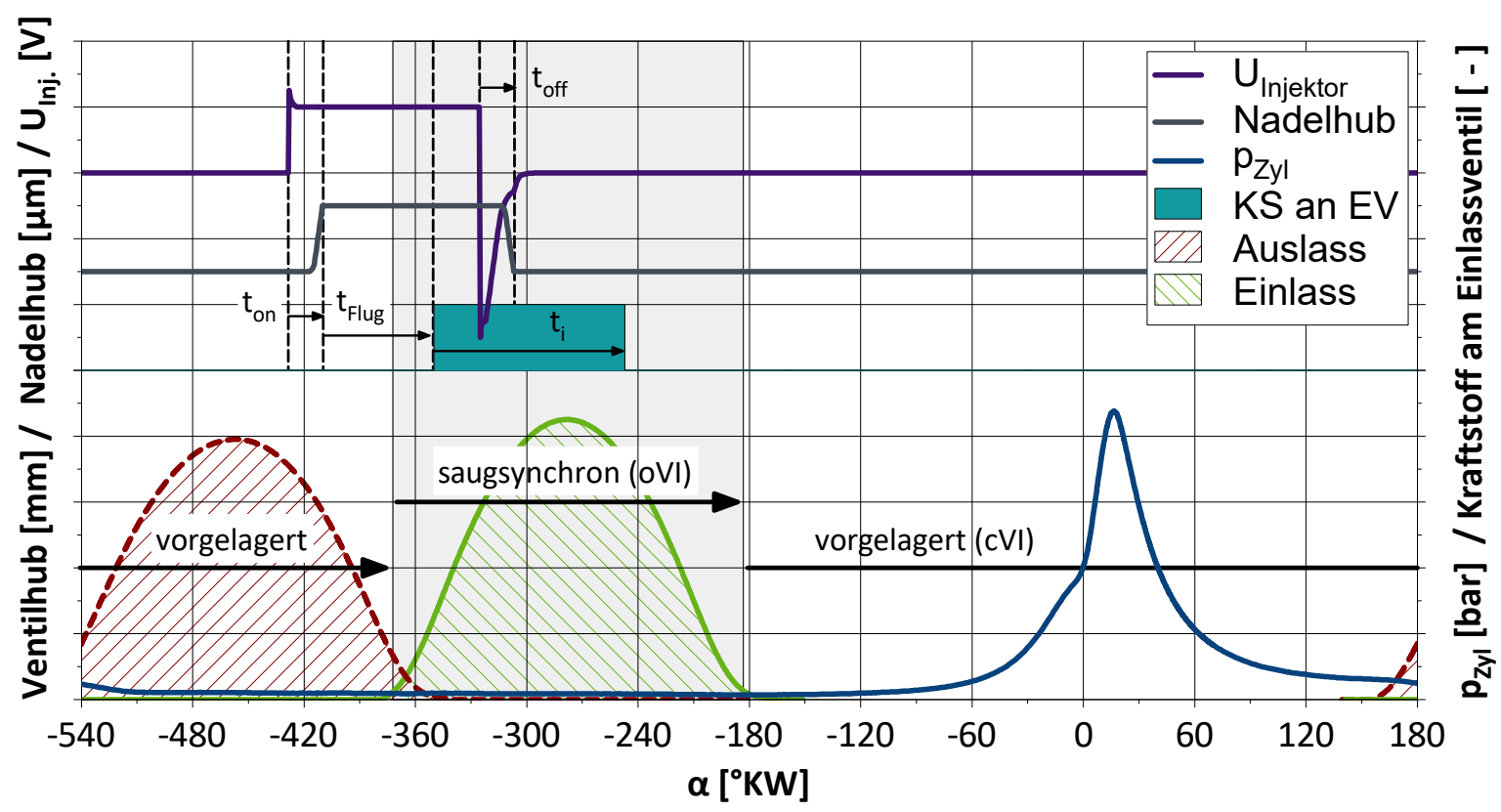

Abbildung 2.3.: Einspritzstrategien mit beispielhafter Darstellung der elektrischen Ansteuerung für eine saugsynchrone Einspritzung

Einlassventile („closed Valve Injection“, cVI) steht verhältnismäßig viel Zeit für die Kraftstoffverdunstung zur Verfügung. Bei später Einspritzung, in die bereits geöffneten Einlassventile (open Valve Injection, oVI), wird in den Saughub des Zylinders eingespritzt, sodass für die Kraftstoffverdampfung bis zur Zündung deutlich weniger Zeit bleibt. Dennoch kann es zweckmäßig sein eine saugsynchrone Einspritzstrategie zu wählen. Zum einen kann die erforderliche Verdampfungsenthalpie des Kraftstoffes eine Kühlung der Zylinderladung und somit eine Reduktion der Klopfneigung herbeiführen. Zum anderen kann bei entsprechend hohen Strömungsgeschwindigkeiten der Ansaugluft Sekundärzerstäubung eintreten [108], was das Tröpfchengrößenspektrum gegebenenfalls günstig beeinflusst [104]. Bei der Parametrierung des Einspritzbeginns muss die Flugzeit $t_{\text {Flug }}$ der Kraftstofftropfen von der Injektorspitze bis in den Brennraum, beziehungsweise zu den Einlassventilen, berücksichtigt werden. Das Erreichen der Einlassventile durch die Kraftstofftropfen ist in Abbildung 2.3 mit einem grünen Balken („KS an EV“) dargestellt. Den Ventilverzugszeiten, also die Dauer zwischen elektrischer Ansteuerung und Nadelöffnen $t_{\text {on }}$ beziehungsweise Nadelschließen $t_{\text {off }}$, müssen ebenfalls im Motorsteuergerät Rechnung getragen werden. 


\subsection{Gemischbildungsmechanismen}

\subsubsection{Primärzerstäubung / Strahlzerfall}

Für den Aufbruch eines Flüssigkeitsstrahls in viele, kleine Tropfen ist Energie notwendig, bei nieder-viskosen Medien wie Kraftstoffen im Wesentlichen zur Überwindung der Oberflächenspannung. Das vorherrschende Zerstäubungsprinzip bei Einspritzdüsen ist die Geschwindigkeitszerstäubung. Die in Form von Kraftstoffdruck gespeicherte Energie wird vom Injektor bei der Einspritzung in das umgebende Medium Luft in kinetische Energie des austretenden Kraftstoffs umgewandelt. Das Prinzip der Lamellenzerstäubung (oder auch Fliehkraftzerstäubung), bei welchem Massenträgheitskräfte für die Zerkleinerung der Flüssigphase verantwortlich sind, ist für die meisten Kraftstoffinjektoren von untergeordneter Bedeutung [30]. Lediglich bei Drall-Injektoren wird dieser Mechanismus neben der Geschwindigeitszerstäubung unterstützend genutzt. Zur mathematischen Beschreibung von Einspritzvorgängen sind unter anderem die dimensionslosen Kennzahlen Reynolds-Zahl Re, Weber-Zahl We und Ohnesorge-Zahl Oh zweckdienlich.

$$
R e=\frac{v d_{S l} \rho_{K r}}{\mu_{K r}} \quad W e_{f l}=\frac{v^{2} d_{S l} \rho_{K r}}{\sigma_{K r}} \quad Z=O h=\frac{\sqrt{W e_{f l}}}{R e}=\frac{\mu_{K r}}{\sqrt{d_{S l} \rho_{K r} \sigma_{K r}}}
$$

Für die Berechnungen herangezogen werden die Austrittsgeschwindigkeit $v$, der Spritzlochdurchmesser $d_{S l}$, die Kraftstoffdichte $\rho_{K r}$, die dynamische Viskosität des Kraftstoffs $\mu_{K r}$ sowie die Oberflächenspannung des Kraftstoffs $\sigma_{K r}$. Die Reynolds-Zahl gibt das Verhältnis von Trägheitskräften und Zähigkeitskräften wieder und eignet sich zur Beschreibung der Strömung im Spritzloch des Injektors. Durch Messungen der intakten Strahllänge ermittelte Ohnesorge [80], dass sich die Zerfallsprozesse mit Hilfe der Weber-Zahl der Flüssigphase beschreiben lässt [7]. Ohnesorge konnte zeigen, dass sich die Primärzerstäubung unter Zuhilfenahme einer neuen Kennzahl $Z$ (heute geläufiger bekannt als die Ohnesorge-Zahl Oh) in charakteristische Bereiche aufteilen lässt.

Diese Erkenntnisse wurden von Reitz und Bracco [91] erweitert, sodass im sogenannten Ohnesorge-Diagramm (siehe Abbildung 2.4a zwischen vier Zerfallsbereichen unterschieden wird. Dem für Kraftstoffinjektoren irrelevanten Bereich des Zertropfens (Rayleigh-Zerfall), den beiden windinduzierten Zerfallsbereichen sowie dem Zerstäuben, oder auch Atomisieren. Hierbei wird allerdings der Einfluss der Gasdichte zunächst vernachlässigt. Nach dem von Reitz in seiner Dissertation [90] erarbeiteten Vorschlag, ist bei Erhöhung der Gasdichte eine Verschiebung der Bereiche nach links unten zu rechnen (siehe Abbildung A.8 im Anhang). Schematische Darstellungen der Zerfallsbereiche aus der Dissertation von Stahl [113] zum Primärzerfall von Druckzerstäubern sind auf der rechten Seite in Abbildung 2.4b dargestellt. Der vorherrschende 


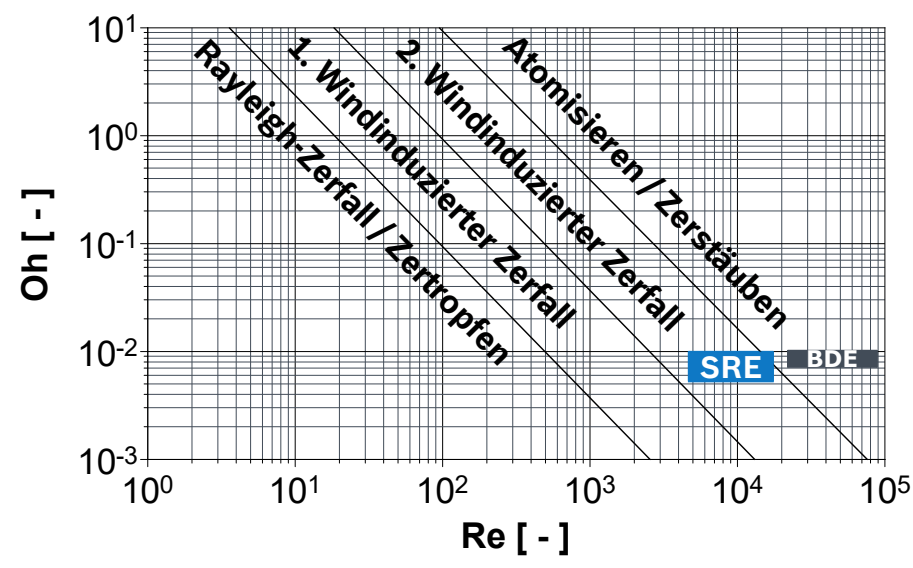

(a)
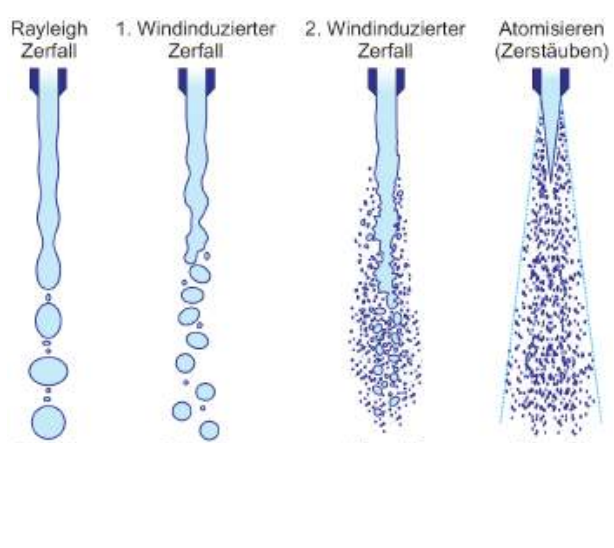

(b)

Abbildung 2.4.: (a) Ohnesorge-Diagramm [80, 91] mit dem für diese Arbeit relevanten Bereich für SRE (blau) und zum Vergleich für BDE (grau) 1 .

(b) Zerfallsmechanismen nach Stahl [113]

Mechanismus für die Tropfenbildung beim Rayleigh-Zerfall sind Schwingungen der Strahloberfläche verursacht durch die Oberflächenspannung. Die resultierenden Tropfen sind größer als der Spritzlochdurchmesser. Beim ersten windinduzierten Zerfall verstärken aerodynamische Kräfte aus der Wechselwirkung mit der umgebenden Luft die Schwingungen, welche zum Strahlzerfall führen. Die Länge des intakten Strahlkerns nimmt ab und die Tropfen sind in etwa so groß wie der Spritzlochdurchmesser. Bei noch höherer Austrittsgeschwindigkeit - im Bereich des zweiten windinduzierten Zerfalls - verkürzt sich der intakte Strahlkern weiter, die aerodynamischen Kräfte werden größer und die Turbulenz der Strömung im Strahl gewinnt an Bedeutung. Dies führt zu Tropfen, die kleiner sind als der Düsendurchmesser. Für noch höhere Strömungsgeschwindigkeiten zerfällt der Strahl direkt am Düsenaustritt in Tropfen, die viel kleiner sind als der Spritzlochdurchmesser. Man spricht vom Zerstäuben oder Atomisieren [61]. Während bei der Benzindirekteinspritzung aufgrund des hohen Kraftstoffdrucks und der somit hohen Austrittsgeschwindigkeit ausschließlich Atomisierung stattfindet, ist bei der Saugrohreinspritzung hauptsächlich der zweite windinduzierte Zerfallsbereich zu erwarten.

Zur Beschreibung eines Tropfengrößenspektrums anhand eines repräsentativen Tropfendurchmessers gibt es verschiedene Ansätze. Für die Bewertung von Kraftstoffsprays aus Injektoren hat sich der Sauter-Durchmesser (SMD) mit $d_{32}=\sum n d_{T r}^{3} / \sum n d_{T r}^{2}$ etabliert, da er das für die Verdunstung relevante Verhältnis von Volumen zur Oberfläche wiedergibt. Der SMD ist stets größer als der geometrische Mittelwert der Durchmesser aller Tropfen $\left(d_{10}\right)$.

\footnotetext{
${ }^{1}$ Annahmen für die überschlägige Berechnung der Bereiche im Ohnesorge-Diagramm: $\sigma_{K r}=0.0197 \mathrm{~N} / \mathrm{m} ; \mu_{K r}=0.41 \mathrm{mPas} ; \rho_{K r}=684-752 \mathrm{~kg} / \mathrm{m}^{3} ; d_{S l, S R E}=125-400 \mu \mathrm{m} ;$ $d_{S l, B D E}=120-220 \mu \mathrm{m} ; p_{K r, S R E}=3-10 \mathrm{bar} ; p_{K r, B D E}=50-200 \mathrm{bar} ; v_{S R E}=20-38 \mathrm{~m} / \mathrm{s}$; $v_{B D E}=110-220 \mathrm{~m} / \mathrm{s}$
} 


\subsubsection{Sekundärzerstäubung / Tropfenzerfall}

In Abhängigkeit der Relativgeschwindigkeit zwischen Luftströmung und Tropfen kann es zu weiterem Tropfenzerfall kommen. Man spricht von Sekundärzerstäubung. Für diesen Fall ist es zielführend nicht die Weber-Zahl des Spritzlochstrahls mit Kraftstoffdichte $\rho_{K r}$, Spritzlochdurchmesser $d_{S l}$ und Austrittsgeschwindigkeit $u$ zu berechnen, sondern die Weber-Zahl des Tropfens im umgebenden Medium Luft mit Luftdichte $\rho_{\text {Luft }}$, Tropfendurchmesser $d_{T r}$ und Relativgeschwindigkeit $v_{r e l}$ aufzustellen.

$$
W e_{T r}=\frac{v_{r e l}^{2} d_{T r} \rho_{L u f t}}{\sigma_{K r}}
$$

Die Weber-Zahl für Tropfen beschreibt das Kräfteverhältnis bestehend aus den aerodynamischen Kräften, welche den Tropfen verformen und den Kräften der Oberflächenspannung, die der Verformung entgegenwirken. Ab einer kritischen Weberzahl $W e_{k r i t .}$ tritt Sekundärzerstäubung auf. Nach Brodkey [10] besteht eine Abhängigkeit ${ }^{2}$ von der Ohnesorge-Zahl. Für sehr kleine $O h<0.1$ - dies ist für Sprays aus Ottokraftstoff typischerweise gegeben - kann der Einfluss der Ohnesorge-Zahl aber vernachlässigt werden. Ab einer Weber-Zahl von 12 kann also mit Sekundärzerstäubung in Form von Schwingungszerfall gerechnet werden, wie auch die Arbeiten von Wierzba [122] und Arcoumanis [3] zeigen. Eine besonders anschauliche Darstellung der Zerfallsmechanismen für steigende Weber-Zahlen liefert die Arbeit von Pilch und Erdmann [84, welche in Abbildung 2.5 zu sehen ist. Für größere Weberzahlen $18<W e<45$ kommt es zum Blasenzerfall. Weitere Zerfallsmechanismen sind Keulen- und Blasenzerfall für $45<W e<100$, Grenzschichtabstreifung für $100<W e<350$ und Wellenabstreifung für $350<W e<2670$. Schließlich kommt es für $W e>2670$ zum Zerfall durch Raleigh-Taylor Instablilitäten.

Allerdings sind die Übergangs-Weber-Zahlen in der Literatur, insbesondere für große $W e>1000$, nicht konsistent. Laut Eichlseder [30] sind für die Gemischbildung in Verbrennungsmotoren in aller Regel aber nur $W e<900$ relevant. Für die Gemischbildung mittels Saugrohreinspritzung sind ohnehin eher die ersten beiden Zerfallsmechanismen von Interesse, verknüpft mit der Frage, ob Sekundärzerstäubung auf dem Weg vom Injektor in den Brennraum überhaupt eintreten kann. Während eines Einspritzvorgangs mit gegebenen Strömungsverhältnissen $\left(v_{r e l} ; \rho_{L u f t}\right)$ und Kraftstoffeigenschaften $\left(\sigma_{K r}\right)$ hängt die Weber-Zahl letztendlich vom einzelnen Tropfendurchmesser ab.

$$
d_{k r i t .}=\frac{12 \sigma_{K r}}{v_{r e l}^{2} \rho_{L u f t}}
$$

Sehr große Tropfen des Tropfenspektrums können nach Schünemann et al. [108] und Samenfink et al. [104] so lange zerfallen, bis ein kritischer Tropfendurchmesser $d_{k r i t .}$ erreicht ist.

\footnotetext{
${ }^{2}$ Kritische Weber-Zahl nach Brodkey [10] We $e_{\text {krit. }}=12\left(1+1.077 \mathrm{Oh}^{1.6}\right)$
} 


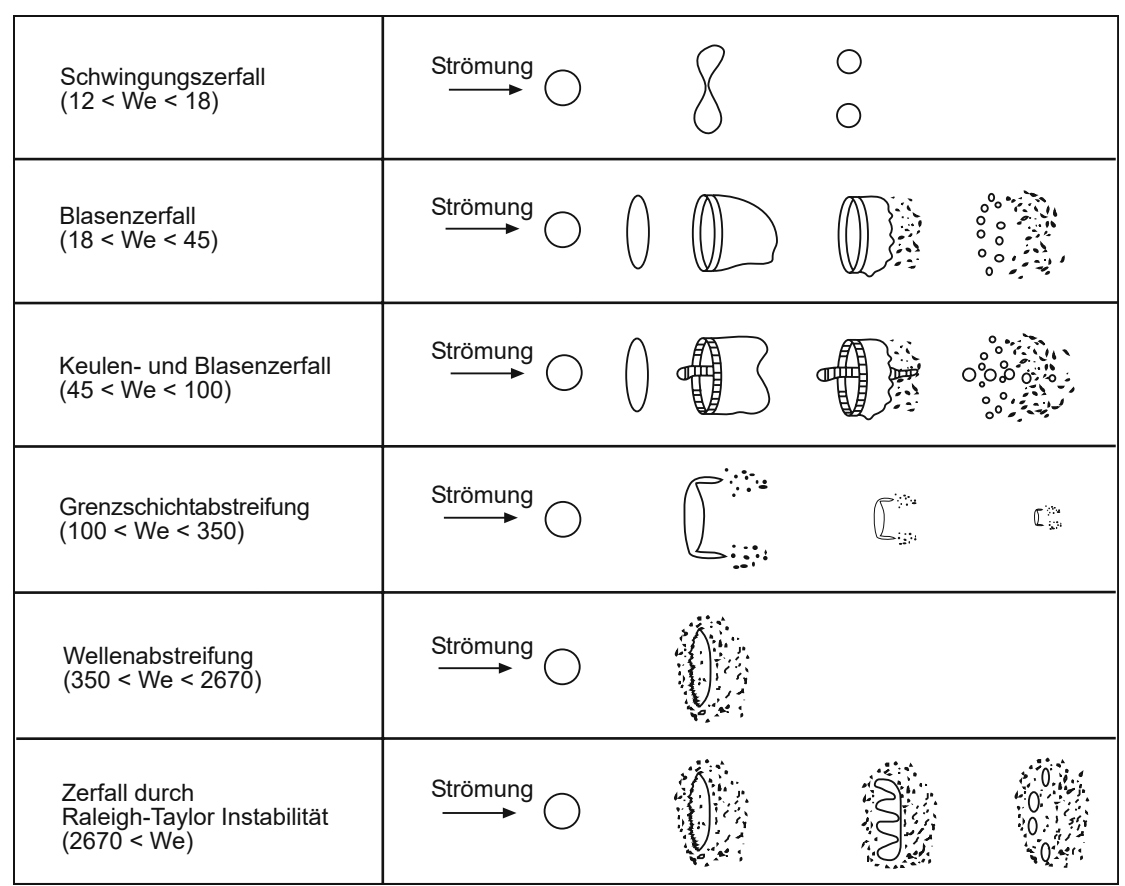

Abbildung 2.5.: Einteilung der Sekundär-Zerfallsmechanismen nach Pilch u. Erdmann 84]

\subsubsection{Wandfilmspeicher \& Wandfilmdynamik}

Hinsichtlich der Gemischbildung stellt der flüssige Kraftstoffwandfilm den ungünstigsten Phasenzustand dar. Ein Anteil des eingespritzten Kraftstoffs gelangt in flüssiger Form an die Saugrohr- und Einlasskanalwände, die Einlassventile sowie in den Brennraum. Im Saugrohr und an den Einlasskanälen bilden sich Kraftstoffwandfilme, die einen unerwünschten Pufferspeicher darstellen. Die in Form von Wandfilmen zwischengespeicherte Kraftstoffmasse ist abhängig von der Saugrohrgeometrie, der Sprayform, dem Tropfengrößenspektrum, dem Kraftstoff- und Saugrohrdruck, der Motordrehzahl und den Temperaturen von Saugrohr, Kraftstoff und Ansaugluft [8, 17, 46].

Bei stationärem Motorbetrieb stellt sich für die Wandfilmmasse im Saugrohr ein Gleichgewicht aus Kraftstoffmasseneintrag aus der Einspritzung (Wandfilm-Aufbau) und Abdampfung oder Filmabriss in die Ansaugströmung zum Zylinder (WandfilmAbbau) ein. Insbesondere bei transienten Lastwechseln kommt es jedoch zur Änderung der Wandfilmmasse und des Kraftstoffmassenstroms aus dem Wandfilm in den Brennraum. Dies führt, ohne geeignete Kompensation, zu Fehlern in der Kraftstoffzumessung in den Zylinder. Bei höherer Motorlast und somit größerer eingespritzter Kraftstoffmasse ergibt sich im Gleichgewichtszustand eine größere, im Saugrohr gespeicherte, Kraftstoffmasse. Folglich muss bei einer Erhöhung der Motorlast nicht nur die größere, im Brennraum benötigte, Kraftstoffmasse eingespritzt werden, sondern auch Kraftstoff für die Erhöhung des Pufferspeichers in Form des Saugrohrwandfilms bereitgestellt werden. Ein einfaches, lineares Modell erster Ordnung zur Berechnung der gespeicherten Wandfilmmasse im Saugrohr stellt das $X-\tau-$ Modell von Aquino 
2] dar. Weitere Autoren wie Bellmann [8] und Russ [97] konnten in ihren Arbeiten zeigen, dass das Modell zur Vermeidung von Gemischabweichungen prinzipiell geeignet ist. Nachteilig ist die Tatsache, dass die beiden Modellparameter für viele verschiedene Motorbetriebszustände experimentell bestimmt werden müssen. Ein deutlich komplexeres Modell wurde von Curtis et al. [17] entwickelt. Auf die Beschreibung dieses und weiterer Wandfilmmodelle wird an dieser Stelle verzichtet und stattdessen auf die Arbeiten von Chevalier [14], Gnanam [38], Maloney [64], Onder [81] und Ye [125] verwiesen.

Zwar sind Wandfilme im Einlasskanal und Saugrohr praktisch nicht vermeidbar, doch kann die Gestalt und Masse des Wandfilms durch die Auslegung des Kraftstoffsprays („Spray-Targeting“) beeinflusst werden. Die Eigenschaften der Gasströmung und der Kraftstofftropfen des Primärsprays sind die wesentlichen Einflussparameter der Wandfilmdynamik [30]. In Abbildung 2.6 sind treibenden Kräfte für eine Wandfilmströmung dargestellt. Die mittlere Strömungsgeschwindigkeit eines Wandfilms ergibt sich aus

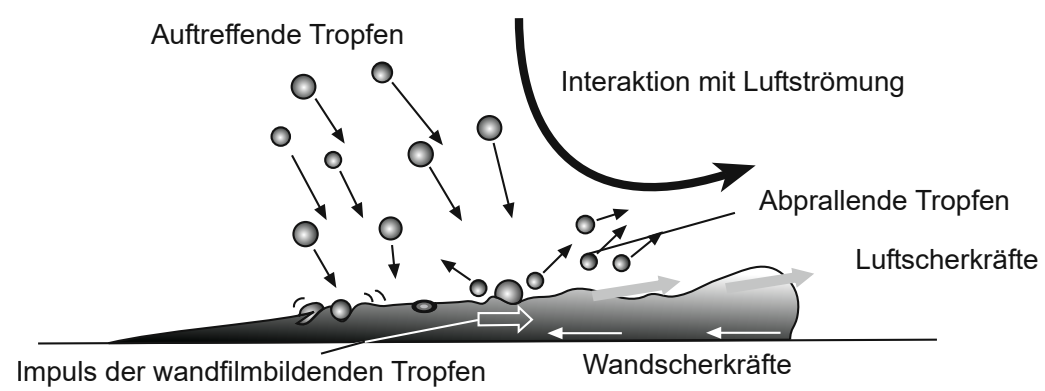

Abbildung 2.6.: Phänomenologisches Modell einer Wandfilmströmung nach [110]

den parallel zur Wand auftretenden Kräften, bestehend aus Luftscherkräften und dem Impulsaustausch der auftreffenden und abprallenden Tropfen an der Phasengrenze, sowie aus der Schubspannung infolge der Wandscherkräfte an der Wandfilmunterseite. In Abhängigkeit vom Verhältnis Tropfengröße zu Wandfilmhöhe, der Tropfengeschwindigkeit, dem Aufprallwinkel und den temperaturabhängigen Stoffeigenschaften des Fluids sind unterschiedliche Tropfen-Wand-Interaktionen zu erwarten. Im Hinblick auf die Aufprallprodukte wird zwischen Tropfenreflexion, Tropfenkoaleszenz und Tropfendestruktion unterschieden. Bei der Spray-Wand-Interaktion, wie sie im Kontext der Saugrohreinspritzung auftritt, ist aufgrund der hohen Tropfengeschwindigkeiten vor allem letzteres, die Tropfendestruktion, relevant. Im Allgemeinen sind dabei die resultierenden Tropfen kleiner als die Originaltropfen des Sprays und der Austrittswinkel ist in der Regel flacher als der Eintrittswinkel [101].

Die Geschwindigkeit der angrenzenden Gasströmung ist in vielerlei Hinsicht von besonderer Bedeutung. So nimmt nach Himmelsbach [47, Elsässer [31] und Ebner [29] die Wandfilmhöhe bei steigender Gasgeschwindigkeit aufgrund der größeren induzierten Schubspannungen ab. Außerdem wird die Fließrichtung des Kraftstoffwandfilms in Saugrohr und Einlasskanal maßgeblich durch die Gasströmung beeinflusst. Ist die Gasströmungsgeschwindigkeit sehr hoch, kann dies nach Elsäßer [31] zu sogenannten 
überkritischen Filmströmungen führen, also zu Tropfenablösungen aus den Wellenkämmen des Wandfilms, insbesondere bei kleinen Strömungsquerschnitten, wie zum Beispiel im Einlassventilspalt bei kleinem Ventilhub. Dieser und weitere, aus Sicht der Gemischbildung kritisch zu bewertende, Filmtransportvorgänge wurden von Costanzo und Heywood beobachtet [16] und sind auszugsweise in Abbildung A.9 im Anhang dargestellt. An einem optisch zugänglichen Einzylindermotor mit quadratischer Kolbenfläche konnte dokumentiert werden, dass schubspannungsgetriebene Wandfilme in den Einlassventilspalt, an das Brennraumdach und an die Unterseite der Einlassventile gelangen können. Dies unterstreicht die Notwendigkeit, die Wandfilmbildung mittels einer gezielten Auslegung der Spraygeometrie („Spray-Targeting“) unter Berücksichtigung von Saugrohr- und Kanalgeometrie und der Darstellung von möglichst kleinen Tropfen durch den Injektor auf ein Minimum zu begrenzen.

\subsubsection{Verdunstung}

Um das Ziel einer idealen, vorgemischten Verbrennung zu erreichen, ist es erforderlich den flüssig eingespritzten Kraftstoff bis zum Zeitpunkt der Entflammung vollständig in den gasförmigen Phasenzustand zu überführen. Dieser Phasenübergang ist durch zwei gleichzeitig ablaufende Vorgänge bestimmt [30].

- Verdunstung - findet statt, sofern die Kraftstofftemperatur unterhalb der Siedetemperatur des Kraftstoffes liegt;

- Verdampfung - ist die Kraftstofftemperatur gleich oder höher als die Siedetemperatur des Kraftstoffes, kommt es zu diesem schnelleren Phasenübergang.

Bei der Saugrohreinspritzung ist bei betriebswarmem Motor aus zweierlei Gründen mit beiden Phasenübergangsarten zu rechnen. Zum einen stellen sich unterschiedliche Kraftstofftemperaturen ein, je nachdem ob ein Tropfen auf die heißen Einlassventile gelangt $\left(T_{E V}<250^{\circ} \mathrm{C}[1]\right)$, auf die Kanalwände mit Motortemperatur trifft $\left(T_{\text {Mot. }} \approx 90^{\circ} \mathrm{C}\right)$ oder sich in der Gasphase der Ansaugluft befindet $\left(T_{L, \text { ans }}<60^{\circ} \mathrm{C}\right)$. Zum anderen erstreckt sich die Siedelinie von Ottokraftstoff über einen Temperaturbereich von etwa $40-200{ }^{\circ} \mathrm{C}$, sodass hochsiedende Anteile verdunsten während niedrigsiedende Anteile bereits verdampfen. Zum Zwecke einer besseren Lesbarkeit wird im folgenden jedoch überwiegend der Begriff Verdunstung verwendet, auch wenn in Abhängigkeit von der Kraftstofftemperatur auch Verdampfung möglich wäre. Der für gewisse Temperatur- und Druckverhältnisse erreichbare Gleichgewichtszustand für das Verhältnis von Gasphase zu Flüssigkeitsphase kann im Motorbetrieb mangels der zur Verfügung stehenden Zeit nicht erreicht werden. Entscheidend für den Phasenübergang ist die Differenz zwischen Sättigungspartialdruck in unmittelbarer Nähe der Flüssigkeitsoberfläche und dem Partialdruck in der Luft [30]. Die wichtigsten Parameter in Bezug auf die Kraftstoffverdunstung sind die Temperaturen von Ansaugluft und Kraftstoff, die Temperaturen der Saugrohr- und Brennraumwände, die Einlassventiltemperatur sowie die zur Verfügung stehende Zeit. Neben der Relativgeschwindigkeit 
zwischen Tropfen und Gasphase spielen die Oberflächen der Zweiphasengrenze, also die Tropfen- und Wandfilmoberflächen, eine entscheidende Rolle [94]. Somit ist ersichtlich, dass kleine Kraftstofftropfen durch die Erhöhung der spezifischen Oberfläche die Verdunstung begünstigen. Die vollständige Verdunstung des Kraftstoffs stellt jedoch, insbesondere bei kaltem Motor - also im Kaltstart sowie dem Motorwarmlauf - eine Herausforderung dar. Eine unvollständige Verdunstung führt somit, aufgrund der Gegenwart von flüssigem Kraftstoff im Brennraum, zu unerwünschten, diffusionsgesteuerten Verbrennungen mit deutlich erhöhten Schadstoffemissionen, wie unter anderem die Arbeit von Rottenkolber et al. zeigt [95].

\section{Tropfenverdunstung}

Beim Verdunstungsvorgang einzelner, umströmter Tropfen, handelt es sich um ein gekoppeltes Wärme- und Stoffübergangsproblem in flüssiger Phase und Gasphase, bei welchem dreidimensional zu beschreibende Strömungen sowohl im Tropfen, als auch der umgebenden Gasphase auftreten. Aufgrund der Komplexität existieren verschiedene Modelle, die sich in ihrer Modellierungstiefe und somit auch im Berechnungsaufwand stark unterscheiden. Für detaillierte Informationen zu den verschiedenen Modellansätzen wird an dieser Stelle auf die Dissertationen von Stengele [114], Gartung [36] und Hermann [44] verwiesen.

Die Simulation der Verdunstung von vielkomponentigen Mischungen wie Ottokraftstoff, welcher aus über 200 verschiedenen Kohlenwasserstoffen besteht, war das Ziel der Dissertation von Gartung [36]. Durch die Formulierung von Verteilungsfunktionen auf Basis der kontinuierlichen Thermodynamik, konnte das Verdunstungsverhalten von Mehrkomponentenmischungen mit hoher Genauigkeit berechnet werden und anhand von Versuchen mit Einzeltropfen und Sprays verifiziert werden. In Abbildung 2.7 ist der Einfluss von Umgebungstemperatur und -druck für Tropfen aus Super Plus Kraftstoff in ruhender Umgebung aufgetragen. Da sich die Verdunstungsdauer nach dem $d^{2}$ Gesetz grundsätzlich reziprok zum Quadrat des Tropfendurchmessers verhält, kommt eine bezüglich des Durchmesserquadrats normierte Ordinate zum Einsatz. Das $d^{2}$ Gesetz beschreibt die lineare Abnahme des Quadrats des Tropfendurchmessers mit der Zeit für eine konstante Tropfentemperatur [39]. Für einen Kraftstofftropfen mit einem für SRE realistischen Anfangsdurchmesser von $d_{T r, 0}=60 \mu \mathrm{m}$ und atmosphärischem Umgebungsdruck ergibt sich beispielsweise bei einer Erhöhung der Umgebungstemperatur von $T_{g}=300 \mathrm{~K}$ auf $400 \mathrm{~K}$ eine Reduktion der Verdunstungsdauer von über $800 \mathrm{~ms}$ auf etwa $72 \mathrm{~ms}$. Neben dem erheblichen Einfluss der Umgebungstemperatur ist auch zu erkennen, dass der Einfluss des Umgebungsdrucks mit steigender Temperatur nahezu verschwindet. Außerdem aus der Dissertation von Gartung hervorzuheben ist die Erkenntnis, dass die Verdunstungsneigung eines Einzeltropfens nicht direkt auf die eines dichten Sprays aus Injektoren für Direkteinspritzung übertragbar ist. Die lokale Anreicherung von Kraftstoffdampf sowie die Abkühlung der Gasphase und der Tropfen hemmen die Verdunstung. Daher ist nicht nur die Zerstäubungsgüte, sondern auch 


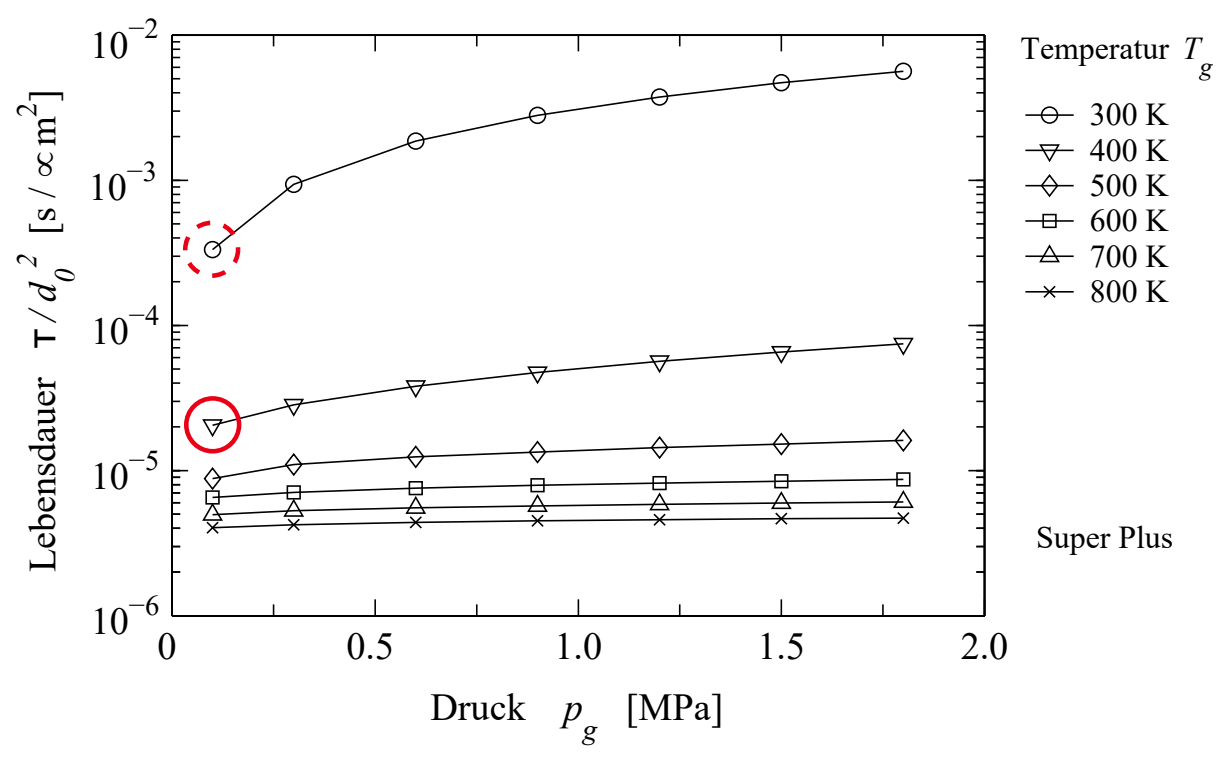

Abbildung 2.7.: Normierte Tropfenlebensdauer als Funktion des Umgebungsdrucks und der Umgebungstemperatur für Tropfen aus Super Plus Kraftstoff; $\left(T_{T r, 0}=\right.$ $293 K ; v_{r e l}=0 \mathrm{~m} / \mathrm{s}$ ) nach Gartung [36]

die geometrische Form des Sprays sowie der Lufteintrag ins Spray („entrainment“) relevant. Vergleichbare Effekte sind auch bei niedrigerem Kraftstoffdruck bei Sprays aus SRE-Injektoren zu erwarten. Wie sich die Spraygeometrie in einem realen Ansaugkanal auf die Kraftstoffverdunstung bei Saugrohreinspritzung auswirkt, ist Bestandteil der vorliegenden Arbeit und wird im Ergebniskapitel 4.1.1 beschrieben.

\section{Wandfilmverdunstung}

Da bei Saugrohreinspritzung die Bildung von Kraftstoffwandfilmen im Einlasskanal häufig nicht vermeidbar ist, wird im folgenden Abschnitt auf die Wandfilmverdunstung eingegangen. Die bereits erwähnte (massen-)spezifische Oberfläche der Phasengrenze eines Wandfilms ist deutlich geringer als die eines Kraftstoffsprays. Während die Phasengrenzfläche eines Sprays von der Tropfengröße und der Tropfenanzahl abhängt, ist für Wandfilme deren Höhe maßgeblich. Die Verdunstungsdauer einer definierten Flüssigkeitsmasse in Form eines Wandfilms ist neben den Stoffeigenschaften im Wesentlichen abhängig von der Wand- und Umgebungstemperatur, dem Umgebungsdruck sowie von der Relativgeschwindigkeit der angrenzenden Gasphase. Die Konzentration des bereits verdunsteten Kraftstoffs in unmittelbarer Nähe zum Wandfilm wirkt sich ebenfalls auf die Verdunstungsrate aus.

Das Verdunstungsverhalten von Kraftstoffwandfilmen, wie sie in Ottomotoren zu erwarten sind, hat Schulz [109] in seiner Dissertation detailliert anhand optischer Messverfahren analysiert. Auch wenn der Fokus der Arbeit auf Wandfilmen im Kontext der Benzindirekteinspritzung liegt, sind die Ergebnisse auch für die Saugrohreinspritzung 
aufschlussreich, da die Untersuchungen von grundlegender Natur sind. Die untersuchten Wandfilme werden in einer konditionierten Spraykammer durch den Wandkontakt eines BDE-Injektorsprays erzeugt. Eine von mehreren optischen Messmethoden, die in der Dissertation von Schulz zur Anwendung kommen ist in Abbildung 2.8 zu sehen. Hierbei wurde mittels Hochgeschwindigkeitsvisualisierung die Verdunstung des Wandfilms auf einer eloxierten Aluminiumplatte erfasst. Die Stoffeigenschaften des Mediums wirken sich einerseits auf die Verdunstungsdauer, andererseits aber auch auf das Fließverhalten des Wandfilms aus. In Abbildung 2.9 sind experimentell ermittelte

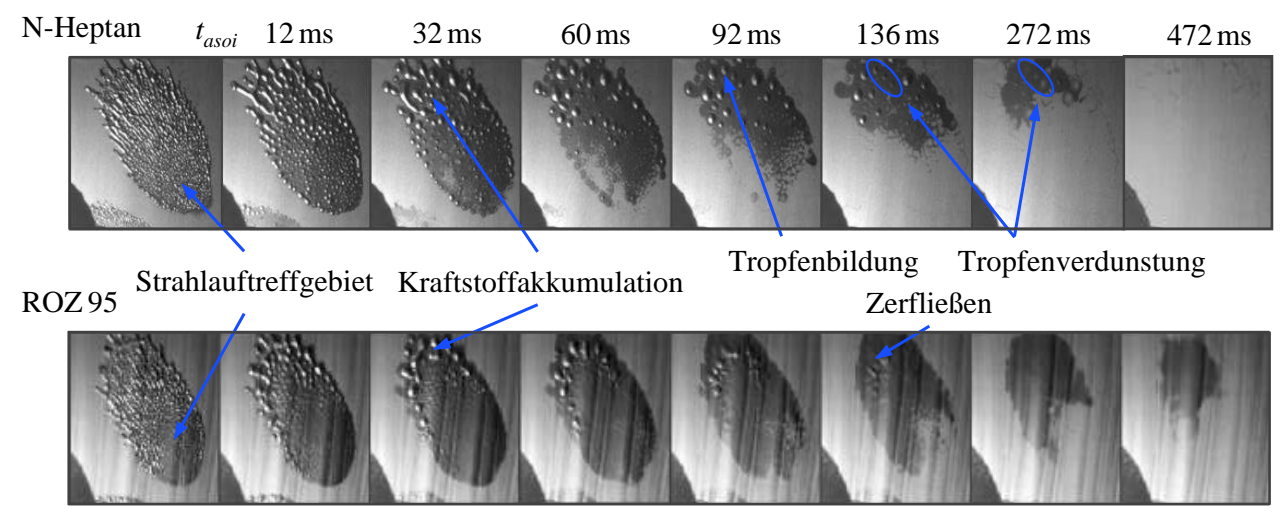

Abbildung 2.8.: Verdunstungsvorgang eines n-Heptanwandfilms mit Tropfenbildung (oben) und eines Benzinwandfilms mit zerfließenden Tropfen (unten) bei $p_{\text {Rail }}=50 \mathrm{bar}, T_{K}=20^{\circ} \mathrm{C}, T_{\text {Wand }}=80^{\circ} \mathrm{C}, v_{\text {rel }}=0 \mathrm{~m} / \mathrm{s}$ [109]

Verdunstungszeiten bei Variation von Spraykammerdruck $p_{\text {Kammer }}$, Kammertemperatur $T_{K}$, Kraftstoffdruck im Rail $p_{\text {Rail }}$ und Kraftstoffmedium auf einer logarithmischen Ordinate dargestellt. Im linken Diagramm wird der erhebliche Einfluss der Kammertemperatur deutlich $\left(T_{K}=T_{\text {Wand }}=T_{0, \text { Gas }}=T_{0, \text { Kraftstoff }}\right)$. Eine Erhöhung von $T_{K}$ um $60 \mathrm{~K}$ führt beim Einsatz von iso-Oktan zu einer Reduktion der Verdunstungsdauer um mehr als Faktor zehn. Eine Erhöhung des Raildrucks ermöglicht unter diesen Randbedingungen eine Verkürzung der Verdunstungszeit, da eine reduzierte Kraftstoffmasse auf der Wand abgeschieden wird. Die Verdunstungsdauer erhöht sich mit steigendem Kammerdruck für iso-Oktan (links), wie auch für einen dreikomponentigen Ersatzkraftstoff und RON95 Ottokraftstoff (rechts) degressiv. Dies ist auf die bei steigendem Druck größere Häufigkeit der zwischenmolekularen Stöße und der damit verbundenen reduzierten Diffusionsgeschwindigkeit in der Gasphase zurückzuführen [36]. Der Abtransport des Kraftstoffdampfes wird somit behindert. Wird bei hoher Kammertemperatur $T_{K}=140{ }^{\circ} \mathrm{C}$ und mittlerem Raildruck $p_{\text {Rail }}=150$ bar handelsüblicher Ottokraftstoff (RON95) eingespritzt, ergibt sich bei atmosphärischem Kammerdruck eine Verdunstungszeit von etwa $150 \mathrm{~ms}$, wie im rechten Diagramm von Abbildung 2.9 $\mathrm{zu}$ sehen ist (schwarzer Kreis). Auch wenn kein direkter Vergleich zur berechneten Tropfenlebensdauer in Abbildung 2.7 aus der Arbeit von Gartung [36] zulässig ist? unterstreicht die mehr als doppelt so lange Verdunstungszeit des Wandfilms unter

${ }^{3}$ RON95 vs. RON98; Umgebungstemperatur von $400 \mathrm{~K}$ vs. $413 \mathrm{~K}$; 

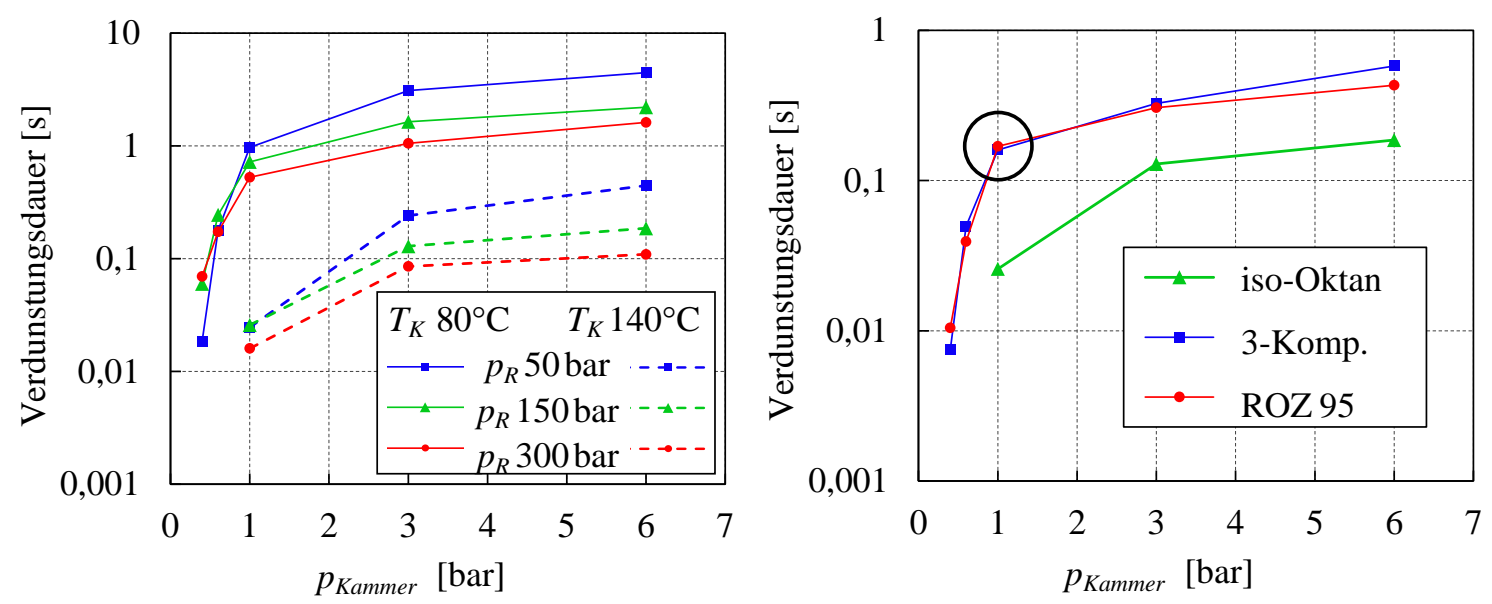

Abbildung 2.9.: Experimentell bestimmte Verdunstungszeiten der Wandfilme bei $v_{\text {rel }}=0 \mathrm{~m} / \mathrm{s}$ aus iso-Oktan (links) und aus iso-Oktan, 3-KomponentenErsatzkraftstoffkraftstoff und ROZ 95 bei $p_{\text {Rail }}=150 \mathrm{bar}$ und $T_{K}=140^{\circ} \mathrm{C}$ (rechts) [109]

ähnlichen Bedingungen die Schwierigkeit der Wandfilmverdunstung im Kontext der ottomotorischen Gemischbildung.

Ein weiterer entscheidender Aspekt der Wandfilmverdunstung ist nach Lenz [62] neben der Umgebungstemperatur die Wandtemperatur. Demnach tritt die schnellste Verdampfung des im Wandfilm gespeicherten Kraftstoffes dann auf, wenn die Wandtemperatur etwa $40-50{ }^{\circ} \mathrm{C}$ über der Siedetemperatur der jeweiligen Kraftstoffkomponente liegt. Ist die Gas- oder Wandtemperatur im Saugrohr indes zu gering, kann bereits verdampfter Kraftstoff wieder kondensieren. Dieser Zusammenhang wurde in der Arbeit von Scherenberg [107] untersucht und anhand einer sogenannten Gemischsättigungstemperatur dargestellt Abbildung A.7). Hervorzuheben ist die Erkenntnis, dass für saugmotorische Volllast und stöchiometrisches Gemisch mit (damals) handelsüblichem Ottokraftstoff die Temperatur von Ansaugluft und Kanalwand nicht unter $33^{\circ} \mathrm{C}$ sein darf, wenn Kondensation vermieden werden soll. Für Motoren mit Aufladung liegt diese Temperatur entsprechend höher. Im Gegensatz zur Kondensation an Wänden ist Kondensation in der Gasphase jedoch als unkritisch einzustufen, da hierbei typischerweise sehr kleine Tröpfchen entstehen $\left(d_{T r}<1 \mu m\right)$. Auch Messungen am Vollmotor stützen den wesentlichen Einfluss der Temperaturen. Messungen von Simon [112] an Motoren mit Zentraleinspritzung und kaltem Motor haben eine im Saugrohr-Wandfilm gespeicherte Kraftstoffmasse von bis zu 10 Arbeitsspielen ergeben. Bei betriebswarmem Motor reduzierte sich dieser Wert auf etwa 3 Arbeitsspiele. Abschließend sei an dieser Stelle noch angemerkt, dass die turbulente Strömung in Saugrohr und Einlasskanal die Verdunstungsneigung von flüssigen Kraftstoffwandfilmen durch Konvektion zusätzlich begünstigt [30]. 


\subsection{Partikel - Eigenschaften und Bildungsmechanismen}

Die für Abgase aus Verbrennungsmotoren relevante Definition für Partikel geht auf die „Richtlinie 70/220/EWG“ des Rates der europäischen Gemeinschaft von 1970 zurück ${ }^{4}$ Danach sind Partikel „Abgasbestandteile, die bei einer Temperatur von max. $52{ }^{\circ} \mathrm{C}$ im verdünnten Abgas mit Filtern [...] abgeschieden werden“ [88. Die wesentlichen Bestandteile von Partikel gliedern sich nach Eastwoods Untersuchungen [28], die hauptsächlich an Dieselmotoren durchgeführt wurden, in die folgenden Kategorien.

- Organischer Anteil (flüchtig / löslich). Mehr als 100 verschiedene Spezies

- Sulfate (flüchtig / löslich). Im Wesentlichen Schwefelsäure und $\mathrm{SO}_{4}^{-2}$ Ionen

- Nitrate (flüchtig / löslich). Wasserlösliche Nitrate, $\mathrm{NO}_{3}^{-}$Ionen und $\mathrm{HNO}_{3}$

- Kohlenstoffhaltige Verbindungen (Feststoff / unlöslich) „Ruß“, aber kein reiner Kohlenstoff

- Asche (Feststoff / unlöslich). Anorganische Elemente und Verbindungen, z.B. aus Additivpaketen des Kraftstoffs oder Öls, sowie metallischer Abrieb

Der jeweilige Anteil der Bestandteile variiert je nach Kraftstoff, Brennverfahren und Motorbetriebspunkt. So sind mit heutigem Ottokraftstoff in Deutschland, der nach DIN EN 228 praktisch frei von Schwefel ist $(<0.001 \%)$, keine nennenswerten Mengen an Sulfaten zu erwarten. Es handelt sich bei den partikelförmigen Emissionen also nicht nur um Feststoffe, sonder auch um flüchtige Bestandteile. Bei der Messung der Partikelanzahl (PN) schreibt der Gesetzgeber in Europa jedoch vor, möglichst alle flüchtigen Anteile in einer geeigneten Vorkonditionierung mit $T_{W a n d}>573 \mathrm{~K}$ zu verdampfen, bevor das Messgas in den Partikelzähler gelangt [27]. Eastwood vermeidet mit der Kategorie „Kohlenstoffhaltige Verbindungen“ bewusst den auch in der Fachwelt geläufigen Begriff „Ruß“, da dadurch oft fälschlicherweise impliziert wird, dass es sich ausschließlich um elementaren Kohlenstoff handelt.

Neben der chemischen Zusammensetzung ist die Partikelgröße ein wesentliches Merkmal der Partikelemission, insbesondere aus Immissionssicht im Sinne der gesundheitlichen Bedeutung. Für die Überprüfung des Partikelanzahlgrenzwerts von $6 \cdot 10^{11} \mathrm{\#} / \mathrm{km}$ ist es erforderlich Nanopartikel ab einer Größe von $d_{P} \geq 23 \mathrm{~nm}$ zu erfassen. Die Gestalt des emittierten Partikelgrößenspektrums ist abhängig von Kraftstoff, Brennverfahren und Motorbetriebsparametern.

Für die Beschreibung der Parikelentstehung bei Verbrennungen haben sich zwei Ansätze etabliert. Zum einen die Elementarkohlenstoffhypothese, welche auch diffusionskontrollierte Rußbildung oder Radikal-Hypothese genannt wird. Zum anderen die Polyzyklenhypothese und dabei im Speziellen die Acetylentheorie. Detaillierte Informationen zu den Prozessschritten der beiden Partikelentstehungshypothesen können

${ }^{4}$ Die Richtlinie 70/220/EWG ist jedoch nicht mehr in Kraft. Die Begriffsdefinition wurde in die aktuellere und gültige „Verordnung (EG) Nr. 715/2007“ 21] aufgenommen 
den Ausführungen von Bockhorn [9], Mansurov [65], Rothe [93], Knauer [55] und Ruschel [96] entnommen werden.

Abbildung 2.10 fasst die wesentlichen Prozessschritte der Rußbildung sowie die notwendigen Bedingungen zu Luftmangel und Temperatur anschaulich zusammen. Neben

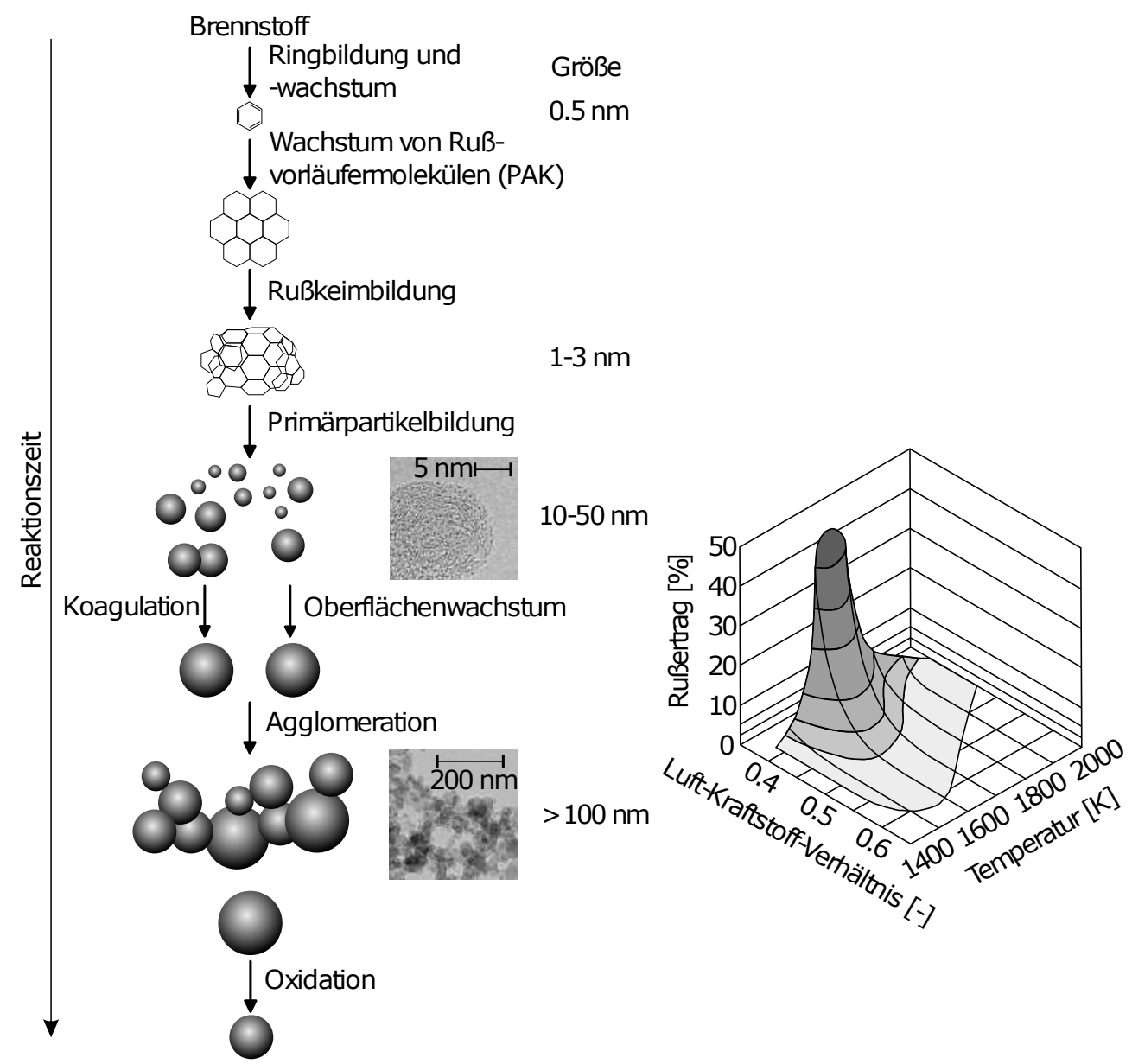

Abbildung 2.10.: Partikelentstehung nach Bockhorn [9] und Mayer [66]

den beiden erwähnten Pfaden der Rußentstehung stellt die Bildung von Sekundärpartikel durch Kondensation von unverbranntem Kraftstoff im Abgas von Verbrennungsmotoren eine weitere Partikelquelle dar. Im Besonderen die hochsiedenden polyzyklischen aromatischen Kohlenwasserstoffe können bei Abkühlung des Abgases kondensieren. Insofern hat die Kraftstoffzusammensetzung nicht nur einen direkten Einfluss auf die Entstehung von Primärpartikel [20, 53], sondern auch auf die Sekundärpartikelbildung. Gerade beim Ottomotor, der auch bei stöchiometrischem Betrieb typischerweise höhere HC-Emissionen aufweist als ein mit Luftüberschuss betriebener Dieselmotor, ist die Möglichkeit der Sekundärpartikelbildung gegeben. Durch die gesetzlich vorgeschriebene Konditionierung der zu analysierenden Abgasprobe, die - wie oben erwähnt - die 
Verdampfung flüchtiger Komponenten vorsieht, spielen die Sekundärpartikel bei der Messung des Partikelanzahlwertes (PN) derzeit keine Rolle.

Zum Abschluss dieses Abschnitts soll auf die Partikelentstehung speziell in Ottomotoren eingegangen werden. Beim Dieselbrennverfahren ist Ruß aufgrund der diffussionskontrollierten Verbrennung als Zwischenprodukt unvermeidbar und, je nach dem, wie vollständig die Rußoxidation noch ablaufen kann, auch als Endprodukt der dieselmotorischen Verbrennung präsent. Bei ottomotorischen Brennverfahren mit LuftKraftstoff-Verhältnis von $\lambda=1$ und homogenem Gemisch, wie bei Saugohreinspritzung oder Direkteinspritzung in den Saughub, sollte es bei warmem Motor im Idealfall keine Verbrennung unter starkem Luftmangel und somit eigentlich keine Partikelemissionen geben. Insbesondere das Brennverfahren Saugrohreinspritzung ist bislang für besonders geringe Partikelemissionen bekannt. Tatsächlich sind aber Stellen im Brennraum mit lokal sehr fettem Gemisch nicht immer vermeidbar. Dies gilt bei der Direkteinspritzung beispielsweise für Kraftstoffwandfilme auf dem Kolben oder an der Injektorspitze, wo der flüssige Kraftstoff bis zum Kontakt mit der Flammenfront nicht vollständig verdunstet und somit lokal, aufgrund der fortschreitenden Kraftstoffverdunstung, eine Zone mit starkem Luftmangel entsteht. An solchen Stellen sind die Bedingungen für diffussionkontrollierte Verbrennung und Rußbildung auch im Ottomotor gegeben. Der Mechanismus für die Partikelentstehnung in einem Ottomotor mit Direkteinspritzung ist beispielhaft in der Arbeit von Kufferath et al. [57] dokumentiert, wie Abbildung 2.11 zeigt. Inwiefern solche Partikelquellen auch bei Ottomotoren mit Saugrohreinspritzung existieren, ist Hauptbestandteil der vorliegenden Arbeit und wird in Kapitel 4.2 ausführlich diskutiert.

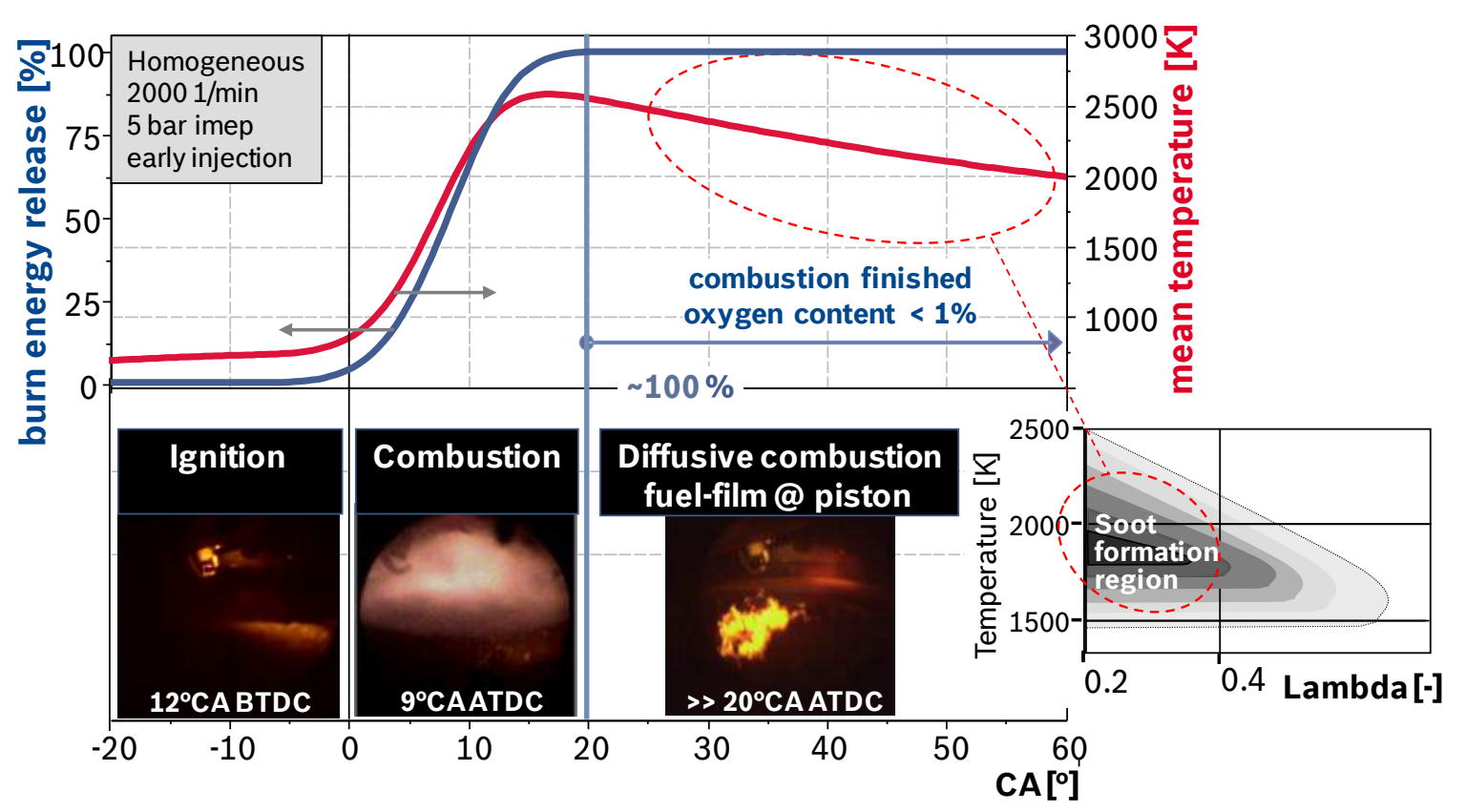

Abbildung 2.11.: Beispielhafte Rußbildung im ottomorischen Prozess mit Benzindirekteinspritzung nach Kufferath et al. [57] 


\subsection{Stand der Technik}

\subsubsection{Analyse der Gemischbildung im Saugrohr}

Bereits im Jahr 1995 dokumentierten Shin et al. [111] die wesentliche Bedeutung der in Abschnitt 2.1 beschriebenen Rückströmung anhand von endoskopisch erstellten HighSpeed Videos im Einlasskanal. Im Rahmen von Untersuchungen zu Kaltstart und Motorwarmlauf wurde erkannt, dass die Rückströmung in den Einlasskanal beim Öffnen der Einlassventile flüssige Kraftstoffwandfilme zurückdrängt und teilweise zerstäubt. Außerdem wird berichtet, dass das heiße, zurückströmende Abgas die Einlasskanäle erwärmt und somit die Verdunstungsbedingungen für den Kraftstoff positiv beeinflusst. Whelen et al. [121] gelang es mittels Laser-Doppler-Anemometrie (LDA) die Tröpfchendurchmesser im Einlasskanal zu messen. Durch den Vergleich der ursprünglichen Tropfengrößen des Injektorsprays $(S M D=200-400 \mu m)$ mit den Tropfendurchmessern infolge der Rückströmung $(S M D=20-80 \mu m)$ konnte eine signifikante Zerkleinerung der Tropfen durch die Rückströmung nachgewiesen werden. Für ein Injektor-Spray mit bereits sehr kleinen Tropfen $(S M D=39 \mu m)$ konnte allerdings keine zerkleinernde Wirkung der Rückströmung mehr festgestellt werden. Diese Feststellung erscheint plausibel, da die Weber-Zahl eines Tropfens für gegebene Strömungsverhältnisse mit dem Tropfendurchmesser abnimmt (siehe Gleichung 2.3) und Sekundärzerstäubung somit weniger wahrscheinlich wird.

\subsubsection{Analyse der Gemischbildung im Brennraum}

Die geläufigste Methode zur Bewertung der Gemischbildung ist die Abgasanalyse. Anhand der Konzentrationen der Abgasbestandteile können Rückschlüsse auf die Qualität der Verbrennung und somit indirekt auch auf die Qualität der Gemischbildung angestellt werden. Konkrete Ursachen, beispielsweise für eine unvollkommene Gemischbildung im Brennraum, können auf diese Art jedoch nicht ermittelt werden. Mit optischen Messverfahren eröffnen sich mehrere Möglichkeiten zur Analyse des Gemischbildungsprozesses, sofern die optische Zugänglichkeit des Brennraumes gewährleistet werden kann. Aufgrund der Vielzahl an Veröffentlichungen in diesem Bereich wird im Folgenden lediglich auf eine Auswahl für das Brennverfahren Saugrohreinspritzung relevanter Arbeiten eingegangen. Eines dieser Messverfahren stellt die Laser induzierte Fluoreszenz (LIF) dar. So hat zum Beispiel Gansert [35] in seiner Dissertation mittels TracerLIF die Kraftstoffverteilung in einer Lichtschnitt-Ebene im Brennraum ermittelt und daraus zweidimensionale Karten des lokalen Luft-Kraftstoffverhältnis berechnet. Daraus konnten Aussagen zur lokalen Gemischhomogenisierung abgeleitet werden. Bei Wensing et al. [120] und Gold et al. [40] war LIF ebenfalls Bestandteil der Untersuchungsmethoden und diente auch in diesen Arbeiten zur Bestimmung der Kraftstoffdampfkonzentration. Darüber hinaus wurden in den Untersuchungen von Gold et al. die Geschwindigkeit der Luftströmung mittels Laser Doppler Velocimetry (LDV) 
an Silikonöltröpfchen und die Größenverteilung und Geschwindigkeiten der Kraftstofftröpfchen mittels Phasen Doppler Anemometrie (PDA) in horizontalen und vertikalen Laserlichtschnittebenen bestimmt. Bei Variationen des Einspritztimings stellte sich heraus, dass an diesem Motor eine frühe saugsynchrone Einspritzung („early oVI“) zu einer guten Kraftstoffdampfverteilung führte. Die PDA kam auch bei den umfangreichen Messungen von Meyer und Heywood zum Einsatz [74 [76]. Mithilfe eines Phasen Doppler Particle Anemometers (PDPA) wurden flüssige Kraftstofftropfen im Brennraum eines Einzylindermotors mit quadratischem Kolben lokalisiert und Rückschlüsse auf Gemischbildungsmechanismen sowohl bei vorgelagerter als auch bei saugsynchroner Einspritzung formuliert.

Die vorgestellten optischen Messverfahren bieten die Möglichkeit für ausgewählte Orte (z.B.: Lichtschnittebene) sehr detaillierte, quantitative Informationen zu gewinnen. Eine ganzheitliche Betrachtung der Vorgänge im Brennraumvolumen, aber auch gleichzeitig an diversen Wänden wie Zylinderlaufbahn und Kolbenoberfläche und den Einlassventilen lässt sich nur schwer realisieren. Für eine solch globale Perspektive in einem thermodynamischen Motor eignet sich, mit der Einschränkung durch das darstellbare Sichtfeld, die High-Speed Video Endoskopie. Mit endoskopischen Zugängen für sichtbares Licht in Saugrohr und Brennraum haben beispielsweise Yumoto et al. [126] die Wechselwirkung zwischen Gemischbildung im Saugrohr und Verbrennung im Brennraum an einem Motorradmotor untersucht. Insbesondere bei saugmotorischer Volllast wurden große Wandfilme im Einlasskanal festgestellt. Verhältnismäßig große, leuchtende Bereiche wurden im Brennraum beobachtet, wenn Teile dieser flüssigen Wandfilme direkt in den Brennraum gelangten. Dieser methodische Ansatz, endoskopische Zugänge sowohl im Saugrohr, als auch im Brennraum zu integrieren, wurde in der vorliegenden Arbeit für eine detaillierte Analyse der Partikelentstehung angepasst und implementiert. Eine weitere Möglichkeit mit üblicherweise hervorragender optischer Zugänglichkeit des Brennraumes bieten Untersuchungen an Transparentmotoren. Beispielhaft dafür sei die Arbeit von Kim et al. [54] erwähnt. Mit einer verhältnismäBig einfachen Papier-Technik konnten Kraftstoff-Wandfilme auf der Zylinderlaufbahn und der Kolbenoberfläche visualisiert und die Wandfilmflächen bestimmt werden. Dadurch war es an diesem ungefeuerten Transparentmotor möglich eine SpraytargetingAuswahl zu treffen, Einspritztimings zu bewerten und Vorteile durch eine Ladungsbewegungsklappe aufzuzeigen. Nachteilig ist die Papierschirmtechnik insofern, als dass sie Wandfilme durch ihre aufsaugende Eigenschaft kumuliert, ein Phänomen welches in einem gefeurten Motor nicht auftreten kann. Es besteht also das Risiko Wandfilme zu überschätzen. Daher wurden lediglich zwei aufeinander folgende Einspritzungen bewertet. Ein ähnlicher Ansatz zur Visualisierung und quantitativen Erfassung von Kraftstoffwandfilmen an einem geschleppten Transparentmotor wird in der vorliegenden Arbeit verfolgt und in Abschnitt 3.2 ausführlich beschrieben. 


\subsubsection{Gemischbildungsoptimierung hinsichtlich gasförmiger Schadstoffemissionen $\left(\mathrm{HC}, \mathrm{CO}, \mathrm{NO}_{x}\right)$}

Die große Herausforderung für die Einhaltung der gesetzlichen Emissionsgrenzwerte für Ottomotoren mit Saugrohreinspritzung waren und sind die gasförmigen Rohemissionen beim Kaltstart und beim anschließenden Warmlauf des Motors bis zur Erreichung der „Light-Off“-Temperatur des Katalsysators. Erst wenn der Katalysator $T_{\text {Kat }} \geq T_{\text {Light-off }}=250-300^{\circ} \mathrm{C}$ erreicht hat [87, werden die Schadstoffe Kohlenmonoxid $(C O)$, Stickoxide $\left(N O_{x}\right)$ und unverbrannte Kohlenwasserstoffe („hydro-carbon“, $\mathrm{HC}$ ) zu $\mathrm{CO}_{2}$ und $\mathrm{H}_{2} \mathrm{O}$ oxodiert, beziehungsweise zu $\mathrm{N}_{2}$ reduziert.

Samenfink et al. [100] gelang eine Reduktion der kumulierten HC-Emissionen um $32 \%$ im Kaltstart und Warmlauf durch eine gezielte Optimierung der Gemischbildung. Neben einer veränderten Spray-Auslegung, unterstützt durch Strömungssimulationen und verifiziert an einem optisch zugänglichen Versuchsmotor, wurden durch Motorprüftstands- und Fahrzeugmessungen Anpassungen von Software und Applikation erarbeitet, um die Startanreicherung zu reduzieren. Die softwareseitige Neuerung sowohl die Einspritzmasse als auch den Einspritzzeitpunkt für mehrere Arbeitsspiele im Kaltstart gezielt vorgeben zu können, trug maßgeblich zum Erfolg bei. In einer späteren Publikation wurden von Samenfink et al. [103] verschiedene Konzepte zur Weiterentwicklung des SRE Injektors untersucht. Das Konzept eines Luft-unterstützten Injektors schied zwar mangels der nötigen Druckdifferenz während des Kaltstarts und wegen Einschränkungen bezüglich des Targetings aus, ein Injektor mit reduzierter Tropfengröße und Heizelement für den Kraftstoff ermöglichte jedoch eine Reduktion der HC-Emissionen um $30 \%$. Stünde ein Kraftstoffdruck von 20 bar zur Verfügung, wären sogar $55 \%$ möglich, allerdings zu erheblich höheren Kosten. In der Arbeit von Frank et al. [37] werden die ersten drei Verbrennungszyklen im Kaltstart detailliert analysiert und hinsichtlich Verbrennungsstabilität und HC-Emissionen optimiert. Es konnte gezeigt werden, dass sich der Injektor mit dem größten $\beta$-Winkel in Verbindung mit dem kleinsten Tropfengrößenspektrum am besten dafür eignet. In der Dissertation von Lang [59] steht neben der ersten Verbrennung des Kaltstart auch die Nachstartphase im Fokus. Ein interessanter Ansatz drei von vier Zylindern fett zu betreiben, durch den vierten Zylinder lediglich Luft zu fördern, um eine stark exotherme Reaktion im Krümmer zur schnellen Aufheizung des Katalysators zu nutzen, führte leider nicht zum Ziel. Zwar konnte die „Light-Off“-Zeit deutlich verkürzt werden, doch die hohen HC-Rohemissionen der drei unterstöchiometrisch betriebenen Zylinder egalisierten diesen Vorteil nahezu vollständig.

Während sich die bislang vorgestellten Arbeiten mit dem Kaltstart befassten, widmete sich Raab [87] in seiner Dissertation der Optimierung des Katalysator-Heizens hinsichtlich HC-Emissionen während des Motorwarmlaufs. Es wurden ausführliche Parameterstudien mit einem variablen Ventiltrieb angestellt und dabei Steuerzeiten, Öffnungsdauer und Ventilhub optimiert. Für diese Arbeit interessant sind die Versuche mit verschiedenen Blenden („Flow Control Valve“, FCV) im Einlasskanal, welche die 
Gemischbildung auf kostengünstige Art und Weise positiv beeinflussen sollen. Zwar wurden durch den Einsatz eines bestimmten FCV Vorteile ermittelt, doch lagen diese bezüglich HC-Emissionen auf vergleichbarem und bezüglich Katalysator Light-Off Zeit auf leicht schlechterem Niveau als das Optimum des variablen Ventiltriebs. In der Arbeit von McGee et al. [67] werden Einspritztiming und Spray-Targeting hinsichtlich HC-Emissionen und Motorlaufruhe untersucht. Als Referenz wird ein vollständig vorgemischtes Luft-Kraftstoff-Gemisch herangezogen. Außerdem werden die anhand des $X-\tau-$ Modells ermittelten Wandfilmmassen in die Bewertung mit einbezogen. So zeigt sich, dass eine saugsynchrone Einspritzstrategie die Wandfilme im Saugrohr deutlich reduzieren kann, die HC-Emissionen mit vorgelagerter Einspritzung aber dennoch geringer ausfallen.

\subsubsection{Partikelemissionen bei Saugrohreinspritzung}

\section{Vergleiche zwischen SRE und BDE}

Auch wenn die Darstellung von Sabathil [98] in Abbildung A.10 im Anhang aus dem Jahr 2011 sicherlich nicht mehr den aktuellen Stand der Motorentechnik wiedergibt, so eignet sie sich dennoch gut, einen Überblick zu erlangen, welche Motoren hinsichtlich Partikelemissionen besondere Herausforderungen darstellen. Interessant für diese Arbeit ist die Tatsache, dass besonders SRE Motoren mit Turboaufladung auffällig hohe Partikelanzahlemissionen aufweisen. Etwa um eine Größenordnung mehr als SRE Saugmotoren ohne Aufladung. Nicht zuletzt deshalb wurden die Untersuchungen der vorliegenden Arbeit an einem Forschungsmotor mit Turboaufladung vorgenommen. Die Aussage, dass Motoren mit Saugrohreinspritzung tendenziell geringere Partikelemissionen aufweisen als Motoren mit Direkteinspritzung, ist in zahlreichen Veröffentlichungen zu finden.

Bei Emissionsmessungen auf Fahrzeugrollenprüfständen mit SRE- und BDE-Fahrzeugen bei $20^{\circ} \mathrm{C}$ kommen unter anderem Ericsson und Samson [33], wie auch Chen et al. [13] in NEDC Zyklen und Chan et al. [12] im FTP75 und US06 Zyklus zu dieser Schlussfolgerung. Wird ausschließlich der Kaltstart des Motors bei sehr niedrigen Temperaturen von etwa $-7^{\circ} \mathrm{C}$ bewertet, werden in den Untersuchungen von Badshah [6] allerdings vergleichbar hohe PN-Emissionen von SRE und BDE-Fahrzeugen gemessen. Bei Fahrzeugmessungen von Zhu et al. [128] im WLTP Zyklus und $-7{ }^{\circ} \mathrm{C}$ liegen die PN-Emissionen des SRE-Fahrzeugs während des Motorwarmlaufs gar über denen des DI-Fahrzeugs. Es gibt also Hinweise auf eine besondere Sensitivität bezüglich kalter Motortemperaturen für die PN-Emissionen bei SRE. 


\section{P-DI - die Kombination von SRE und BDE}

Kommen sowohl Saugrohreinspritzung (SRE bzw. PFI) als auch Direkteinspritzung (BDE bzw. DI) am selben Motor zum Einsatz, spricht man bei diesem Dual-System von „P-DI“. Ziel ist es dabei, die jeweiligen Vorteile der beiden Brennverfahren geschickt miteinander zu kombinieren. So werden beispielsweise in der Arbeit von Ikoma et al. [52] an einem V6-Motor mit Direkteinspritzung die Vorteile durch SRE hinsichtlich der Gemischhomogenisierung bei geringer Ladungsbewegung genutzt, um nicht auf eine Einlasskanalvariante verzichten zu müssen, die hinsichtlich Zylinderfüllung und Strömungswiderstand optimiert ist. Untersuchungen an P-DI Motoren sind für diese Arbeit relevant, da vergleichende Bewertungen der beiden Brennverfahren hinsichtlich der Partikelemissionen am selben Motor besonders aussagekräftig sind. In der Dissertation von Hemminger [43] konnten die erwarteten Vorteile von sehr geringen Partikelemissionen mit SRE bei kleiner Motorlast gegenüber dem Betrieb mit Direkteinspritzung, durch Messungen an einem V8 Motor mit Turboaufladung, aufgezeigt werden. Mit einer kombinierten Einspritzstrategie durch beide Systeme im selben Arbeitsspiel konnten jedoch keine weiteren Vorteile dargestellt werden. Auch bei den Untersuchungen von Su et al. [116] an einem 2.01 Vierzylindermotor mit Direkt- und Saugrohreinspritzung wurde ein erheblicher Vorteil hinsichtlich Partikelanzahlemissionen mittels Saugrohreinspritzung dokumentiert. Bei effektiven Mitteldrücken von 6 bis 8 bar lag die Partikelanzahlkonzentration, bei betriebswarmem Motor mit SRE, um etwa eine Größenordnung (Faktor 10) unter der mit BDE. In der Arbeit von Graf et al. [41] gelang es, die PN Emissionen bei kaltem Motor und transienten Lastsprüngen, durch eine kombinierte Einspritzstrategie mit beiden Einspritzsystemen, erheblich zu senken. Ein detailliertes Verständnis der Partikelentstehung, speziell im Hinblick auf den Beitrag durch die Saugrohreinspritzung, konnte aber noch nicht erarbeitet werden. Hierzu liefert die vorliegende Arbeit neue Erkenntnisse.

\section{Partikelentstehung bei Saugrohreinspritzung}

Das Brennverfahren Saugrohreinspritzung ist allgemein dafür bekannt, im Vergleich zur Direkteinspritzung besonders geringe Partikelemissionen zu ermöglichen. Dennoch sind in der Literatur einige Untersuchungen zu Partikelemissionen zu finden. Gupta et al. [42] zeigen in ihrer Arbeit den Einfluss von Drehzahl und Motorlast auf PN anhand von Messungen an einem SRE-Motor mit 1.01 Hubraum ohne Aufladung. Wie zu erwarten ist, steigen die PN-Emissionen sowohl mit höherer Motorlast als auch mit zunehmender Drehzahl an. In einer Parameterstudie von Arsie et al. [4] werden die Auswirkungen von Abgasrückführungsrate (AGR), Luft-Kraftstoffverhältnis $(\lambda)$ und Zündwinkel auf die Partikelemissionen untersucht. Während eine Gemischabmagerung auf $\lambda=1.13$ erheblich geringere PN-Emissionen ermöglicht, führt eine Anfettung bis zu einem Luftkraftstoffverhältnis von $\lambda=0.93$ nur zu geringfügiger Erhöhung der PN. Eine AGR-Rate von $20 \%$ steigerte die Partikelemissionen gegenüber dem Betrieb ohne 
AGR merklich. Dies wurde den durch die Ladungsverdünnung reduzierten Temperaturen im Brennraum sowie dem geringeren Sauerstoffgehalt im Zylinder zugeschrieben. Der Einfluss des Zündwinkels auf PN war für verschiedene Motordrehzahlen nicht konsistent und verhältnismäßig gering.

Um den Zusammenhängen der Partikelentstehung bei Saugrohreinspritzung näherzukommen, ist neben der Abgasanalyse die Anwendung von optischer Messtechnik ein naheliegender Schritt. Witze und Green [123] stellen in Ihrer Arbeit Untersuchungen an einem durch einen Glaskolben optisch zugänglichen Einzylindermotor vor und werten neben dem LIF-Signal von Kraftstoffwandfilmen auch das Rußeigenleuchten aus. Bei Messungen am kalten Motor, unmittelbar nach dem Kaltstart, wurden stark ausgeprägte Diffusionsflammen („Pool-fires“) erfasst. Es wurde sowohl der Einfluss einer Injektorsprayvariation als auch der des Einspritztimings bewertet. Während eine vorgelagerte Einspritzstrategie (cVI) vorwiegend Wandfilme und Rußleuchten im Bereich der Einlassventile aufwies, waren bei saugsynchroner Einspritzung (oVI) Bereiche im gesamten Brennraum betroffen. Die Ergebnisse des Fluoreszenzsignals aus Kraftstoffwandfilmen spiegelte sich nicht immer in Form von Rußleuchten wieder. Da auf die notwendige Gemischanfettung in der Startphase des Motors verzichtet wurde, ist mehr Sauerstoff im Brennraum als typischerweise bei einem seriennahen Motorstart und Warmlauf zu erwarten wäre. Daher sind die erfassten Diffusionsflammen vermutlich deutlich ausgeprägter, intensiver und langlebiger als in der Realität. Eine Korrelation der optischen Analysen mit Partikelemissionen fand nicht statt, da weder Partikelmasse noch Partikelanzahl im Abgas gemessen wurden.

Weitere, für die vorliegende Arbeit wertvolle Hinweise auf die Partikelentstehung mit SRE liefern die Untersuchungen von Merola et al. 69 73]. Auch hier kommt ein Einzylindermotor mit transparentem Kolbenboden zum Einsatz, welcher allerdings auch für Licht im UV Bereich durchlässig ist. Dadurch ist neben dem Rußleuchten auch die Flammenfront erfassbar. Wie schon bei Witze und Green treten auch in den Studien von Merola et al. Diffusionsflammen häufig im Bereich der Einlassventile auf. Hinsichtlich der Einspritzstrategien erweisen sich hier allerdings saugsynchrone Einspritzzeitpunkte (oVI) als vorteilhaft. Auch die Tatsache, dass eine Erhöhung des Ladedrucks zu scheinbar weniger stark ausgeprägten Diffusionsflammen führt, steht im Widerspruch zu den Erwartungen, da sich die Verdunstungsbedingungen mit steigendem Druck verschlechtern (siehe 2.2.4). Die in [69] und [70] ermittelten Vorteile durch die Anwendung einer Dreifacheinspritzung im saugsynchronen Zeitfenster gegenüber einer konventionellen, vorgelagerten Einfacheinspritzung, verdeutlichen das Potenzial einer sorgsamen Abstimmung der Einspritzstrategie. In Kapitel 4.2.9 wird das Potenzial einer Mehrfacheinspritzung mit SRE als Bestandteil der vorliegenden Arbeit mit einem anderen Ansatz analysiert. Beim Vergleich zweier Injektorvarianten konnte ein deutlicher Vorteil eines Injektors mit 10 Spritzlöchern gegenüber einer Variante mit drei Löchern ermittelt werden [72]. Die Möglichkeit über die Auslegung von Injektor- und Sprayeigenschaften die Partikelbildung innermotorisch zu reduzieren, wird ebenfalls in der vorliegenden Arbeit verfolgt und in Kapitel 4.2.6 ausführlich diskutiert. 


\subsection{Zielsetzung der Arbeit}

Die teils widersprüchlichen Informationen aus der Literatur zum Thema Partikelemissionen mit Saugrohreinspritzung müssen aufgearbeitet und eingeordnet werden. Einerseits wird von erheblichen Vorteilen durch die Saugrohreinspritzung gegenüber der BDE im Hinblick auf die PN-Emission berichtet, andererseits sind auch Hinweise auf nennenswert hohe Partikelemissionen dokumentiert. Im Rahmen der vorliegenden Arbeit soll daher ein umfassendes Verständnis zur Partikelentstehung bei Ottomotoren mit Saugrohreinspritzung aufgebaut werden. Es gilt unter anderem zu verstehen, unter welchen Randbedingungen und bei welchen Motorbetriebspunkten problematisch hohe Partikelemissionen vorliegen, und unter welchen Umständen besonders niedrige PN-Emissionen erzielbar sind.

Des weiteren soll mittels optischer Messtechnik eine Methode entwickelt werden, mit deren Hilfe die Partikelentstehung im Brennraum erforscht werden kann. Da Kraftstoffwandfilme im Brennraum auch bei SRE als typische Ursache für die Partikelentstehung zu erwarten sind, werden darüber hinaus Untersuchungen zur Visualisierung und Quantifizierung von Wandfilmen angestrebt. Erkenntnisse aus Experimenten mit optischer Messtechnik sollen stets mit Partikelanzahlmessungen an einem thermodynamischen Motor verifiziert werden.

Im Sinne eines ganzheitlichen Verständnisaufbaus zur Partikelentstehung mit Saugrohreinspritzung soll sowohl die Gemischbildung im Einlasskanal als auch die Gemischbildung im Brennraum analysiert und optimiert werden. Hierfür gilt es die zur Verfügung stehenden innermotorischen Variationsparameter wie beispielsweise die Auslegung des Injektorsprays, die Einspritzstrategie sowie die Ladungsbewegung hinsichtlich ihres Einflusses auf die Partikelemission zu charakterisieren, um Optimierungsansätze ableiten zu können.

Bei der Analyse von Fahrzyklusmessungen, wie beispielsweise von Samenfink et al [103] veröffentlicht (siehe Abbildung A.11 im Anhang), ist erkennbar, dass gasförmige Schadstoffemissionen (z.B. HC) wirkungsvoll vom Katalysator konvertiert werden können, sobald dieser seine „Light-Off“-Temperatur erreicht hat. Die Erarbeitung von Maßnahmen zur Reduzierung der gasförmigen Schadstoffemissionen ist daher vorwiegend im Kaltstart und während des Katalysatorheizens sinnvoll. Bei Partikelanzahlmessungen, wie beispielsweise in Abbildung A.12 im Anhang aus der Veröffentlichung von Zhu et. al [128], treten hohe PN-Emissionen hingegen nicht nur im Kaltstart und Motorwarmlauf auf, sondern auch bei warmem Motor. Aus diesem Grund soll die Partikelentstehung im Rahmen dieser Arbeit sowohl bei warmem Motor als auch bei kaltem Motor analysiert werden. Bei heutigen Fahrzyklen und EU6-RDE-Messungen erfolgt die Abfahrt innerhalb kurzer Zeit nach dem Motorstart. Daher ist der Emissionsbeitrag aus den Betriebsarten Kaltstart und Katalysatorheizen im Leerlauf, bezogen auf den gesamten Zyklus, eher gering. Aus diesem Grund werden diese beiden Sonderbetriebsarten lediglich in einem Exkurs im Anhang hinsichtlich der PN-Emission, aber auch bezüglich der gasförmigen Rohemissionen untersucht. 


\section{Versuchsträger und Methoden}

Bei der Planung und auch im Verlauf der Untersuchungen zeigt sich, dass je nach zu beantwortender Fragestellung spezifische Anforderungen an einen Versuchsaufbau zu stellen sind. Für die Untersuchung von grundlegenden Phänomenen der Kraftstoffverdunstung im Einlasskanal beispielsweise ist ein gefeuerter Vollmotor als Versuchsträger ungeeignet. Die komplexe Gasdynamik beim Ladungswechsel und die sehr schwer messbare Temperatur der Einlassventile sind nur zwei Beispiele für wesentliche, aber schlecht beherrschbare Einflussgrößen auf die Kraftstoffverdunstung.

Daher werden die Untersuchungen zur Analyse der Kraftstoffverdunstung in einer eigens dafür konzipierten Verdunstungskammer durchgeführt, die in Kapitel 3.1 vorgestellt wird. Auch die Fragestellung nach Ort, Ausdehnung und Verhalten von Kraftstoffwandfilmen innerhalb des Brennraums ist mit Untersuchungen an einem Vollmotor nur sehr eingeschränkt zu beantworten.

Eine deutlich bessere optische Zugänglichkeit für Experimente zu diesem Thema bietet zum Beispiel ein geschleppter Transparentmotor mit Glaszylinder, wie in Kapitel 3.2 beschrieben. Hierbei müssen im Gegenzug anderweitige Einschränkungen, wie beispielsweise unrealistische Wandtemperaturen an den Einlassventilen und im Brennraum, in Kauf genommen werden.

Die tatsächliche Wirksamkeit von Verbesserungsmaßnahmen im Kontext der Gemischbildung mit Saugrohreinspritzung, sei es eine verbesserte Spray-Auslegung, optimierte Einspritz-Timings oder eine veränderte Ladungsbewegung, können wiederum an einem gefeuerten Vollmotor mit entsprechender Messtechnik zur Analyse der Schadstoffemissionen am besten bewertet werden. Nur am gefeuerten, Serien-nahen Vollmotor sind alle wesentlichen Randbedingungen gegeben, wie beispielsweise die realen Temperaturverhältnisse in Einlasskanal und Brennraum, um belastbare Aussagen hinsichtlich der Schadstoffemissionen treffen zu können. Der Versuchsaufbau hierzu wird in Kapitel 3.3 erläutert.

An dieser Stelle sei hervorgehoben, dass alle drei Versuchsträger auf demselben Vollmotor basieren. Sowohl die Geometrie des Einlasskanals der Verdunstungskammer, als auch Zylinderkopf und Kolben des Transparentmotors stammen vom gleichen Vollmotor, welcher, mit endoskopischen Zugängen ausgestattet, am Motorprüfstand zum Einsatz kommt. Dadurch sind sehr gute Voraussetzungen für eine Übertragbarkeit der Erkenntnisse der jeweiligen Experimente geschaffen. 


\subsection{SRE Verdunstungskammer}

\subsubsection{Versuchsaufbau SRE Verdunstungskammer}

Für erste, grundlegende Untersuchungen der Gemischbildung im Einlasskanal wird eine sogenannte Verdunstungskammer konzipiert. Zur Bewertung der Verdunstungsneigung eines SRE-Sprays kommt, im Unterschied zu üblicherweise großvolumigen Spraykammern, eine exakte Nachbildung eines Einlasskanals zum Einsatz. Dadurch soll der signifikante Einfluss von Spray-Wand-Interaktionen auf die Gemischbildung berücksichtigt werden. Ziel ist es, anhand von Druck- und Temperaturmessungen in der Gasphase des abgeschlossenen Volumens der Kammer, Rückschlüsse auf das Verdunstungsverhalten unterschiedlicher Primärsprays verschiedener Injektoren zu erlangen.

Folglich wird die Geometrie des Einlasskanals eines Zylinders, des in dieser Arbeit verwendeten Vollmotors, mittels 3D-Druck aus Aluminium gefertigt. Der Verlauf der Systemgrenze sowie die Anordnung von Injektor und Sensoren ist in Abbildung 3.1 dargestellt. Der Einlasskanal wird stromaufwärts des Injektors (e) durch eine Platte

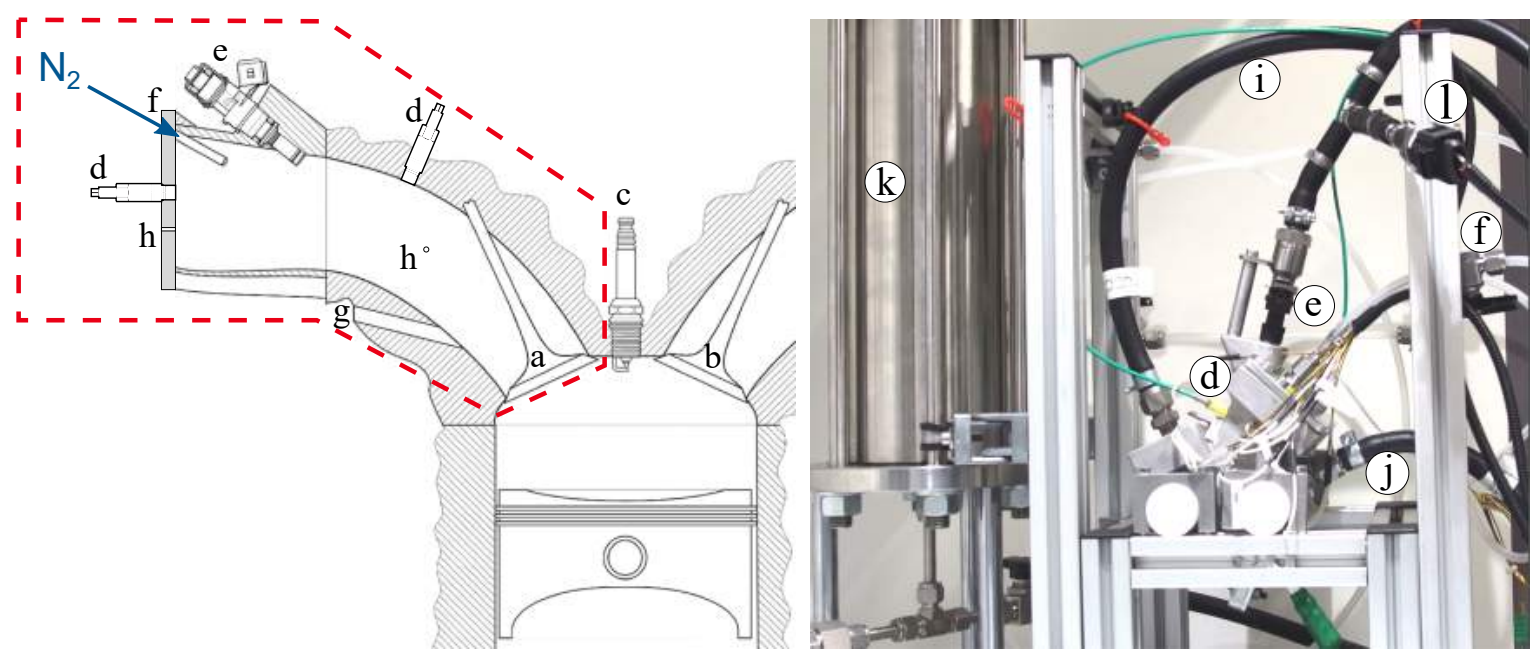

Abbildung 3.1.: Schema der Verdunstungskammer mit Systemgrenze in rot (links); Foto des Versuchsaufbaus (rechts)

verschlossen, in welche, neben Drucksensor (d) und Temperatursensor (h), auch ein verschließbarer Zugang (f) für die Einbringung von Stickstoff $\left(N_{2}\right)$ integriert ist. Um die Bildung eines explosionsfähigen Gemisches zu vermeiden, wird die Kammer stets mit Stickstoff anstelle von Luft befüllt. Stromabwärts ist die Kammer durch die Einlassventile (a) verschlossen. Die Zündkerze (c) und die Auslassventile (b) sind kein Bestandteil der Verdunstungskammer, da sie außerhalb der Systemgrenze liegen. 
Zur Spülung der Kammer mit Stickstoff vor und nach einer Messung kann ein weiterer Zugang (g) geöffnet werden. Zur Einstellung einer definierten Temperatur für Kammerwände und Gasphase kann der Einlasskanal mit einer Konditionierflüssigkeit umströmt werden. Die Leitungen und Anschlüsse für den Vorlauf (j) und Rücklauf (i) der Konditionierung sind auf der rechten Seite von Abbildung 3.1 zu sehen. Der Kraftstoffdruck wird mit Hilfe eines Druckspeichers (k) bereitgestellt. Dazu wird die im unteren Teil des Speichers befindliche Kraftstoffsäule von oben durch Stickstoff mit 3, 0 bar beaufschlagt. Der gewünschte Kraftstoffdruck wird mit einem vorgeschalteten Manometer kontrolliert und mittels eines Kraftstoffdrucksensors (l) zusammen mit allen weiteren Messdaten elektronisch protokolliert.

Zur Erfassung des Kammerdrucks kommen zwei piezoelektrische Absolutdrucksensoren von Kistler zum Einsatz, da die in der Motorentwicklung etablierte Indiziermesstechnik den Anforderungen hinsichtlich der zeitlichen Auflösung gerecht wird. In der Verschlussplatte ist ein Quarz vom Typ 4011A und in der Oberseite des Einlasskanals ein Quarz vom Typ 4007C installiert. Beide Sensoren erfassen Drücke im Bereich von $0-5$ bar. Zur Auswertung der Drucksignale bedarf es eines piezoresistiven Verstärkers. Hierzu wird ein Verstärker vom Typ 4665 verwendet, der ebenfalls von Kistler stammt. Die begleitende Temperaturmessung in der Gasphase erfolgt durch Thermoelemente vom Typ K, welche durch eine Paarung an der Messspitze von Nickel-Chrom mit Nickel-Aluminium gekennzeichnet sind (NiCr-NiAl). Für ein möglichst spontanes Ansprechverhalten der Thermoelemente soll die thermische Trägheit möglichst gering gestaltet werden. Daher wird die Ummantelung der Messspitze der, mit einem Durchmesser von $0,35 \mathrm{~mm}$ bereits recht dünn ausgeführten, Thermoelemente durch Schleifen entfernt. Dabei gilt es besondere Vorsicht walten zu lassen, um den darunter befindlichen Thermofühler nicht zu beschädigen. Wie in einer im Rahmen dieser Arbeit betreuten Masterarbeit von Hoffmann [48] ermittelt wurde, kann dadurch die Ansprechzeit, gekennzeichnet durch $t_{90}$, um $42 \%$ auf $20 \mathrm{~ms}$ reduziert werden. Zur Verstärkung der Thermospannung und zur Kaltstellenkompensation kommt das Modell EGT-K der Firma Reveltronics zum Einsatz. Die Datenerfassung, die Steuerung der Messung sowie die Ansteuerung des Injektors erfolgt durch die Software LabVIEW von National Instruments in Kombination mit einem Messsystem vom Typ cDAQ9188, welches mit einem A/D-Wandler vom Typ NI 9920 und einer Karte für DigitalAusgänge vom Typ NI 9474 bestückt ist.

Obwohl es sich bei diesem Versuchsaufbau um ein verhältnismäßig einfaches Experiment handelt, ermöglicht es dennoch Aussagen zur Verdunstungsneigung von unterschiedlichen Sprayauslegungen. Mittels einer Druckverlaufsanalyse (DVA), welche in Unterabschnitt 3.1.3 noch näher beschrieben wird, kann eine abstrakte Spraybewertung erfolgen. 


\subsubsection{Vorgehensweise für Verdunstungsmessungen}

Die Verdunstungskammer wird stets mit reinem Stickstoff $\left(N_{2}\right)$ befüllt. Dies dient der Sicherheit bei der Versuchsdurchführung, da durch die Verdrängung von Luftsauerstoff kein explosionsfähiges Gasgemisch entstehen kann. Ein willkommener Nebeneffekt ist die Vereinfachung sämtlicher Berechnungen durch den Einsatz von reinem Stickstoff anstelle von Luft. Als Ersatzkraftstoff kommt n-Heptan $\left(C_{7} H_{14}\right)$ zum Einsatz. Auch hierdurch ergeben sich vielerlei Vorteile bei anschließenden Berechnungen. Ottokraftstoff ist, wie in Kapitel 2 beschrieben, ein Gemisch aus mehr als 200 verschiedenen Kohlenwasserstoffen. Die Berücksichtigung der Stoffeigenschaften und Mischungsverhältnisse aller Kraftstoffkomponenten wäre sehr aufwändig und erscheint für die angestrebten Grundlagenversuche nicht verhältnismäßig. Der Kraftstoffdruck wird für alle Versuche an der Verdunstungskammer auf 3,0 bar eingestellt. Die im Vorfeld ermittelten Durchflusskennlinien der untersuchten Injektoren beschreiben die Einspritzmasse pro Nadelhub $\left(q_{d y n}\right)$ als Funktion der elektrischen Ansteuerdauer $\left(t_{e}\right)$ und wurden ebenfalls bei einem Kraftstoffdruck von 3,0 bar und mit n-Heptan ermittelt. Somit ist für eine definierte Ansteuerdauer die in die Kammer eingespritzte Masse bekannt. Der Kammerduck wird zu Beginn einer Messung auf $p_{0}=1000$ mbar eingestellt.

Für eine möglichst gute Reproduzierbarkeit der Messergebnisse wird eine Vorgehensweise angewandt, wie sie in den studentischen Abschlussarbeiten von Hoffmann [48] und Lang [60] erarbeitet wurde:

1. Konditionierung der Kammer auf Zieltemperaturen $35^{\circ} \mathrm{C}$ oder $93.5^{\circ} \mathrm{C}$

2. Spülen der Kammer für mindestens fünf Minuten mit $N_{2}$, zur Abtrocknung von Kraftstoffrückständen

3. Leckagemessung ohne Einspritzung bei $p_{\text {Kammer }}=1500$ mbar und 300 mbar zur Prüfung, ob $\dot{p}_{\text {Leckage }}<0.75 \mathrm{mbar} / \mathrm{min}$

4. Messdatenerfassung starten, Einspritzung von $36 \mathrm{mg}$ n-Heptan ${ }^{1}$

Da nicht alle Injektormuster denselben statischen Durchfluss $\left(q_{\text {stat }}\right)$ aufweisen, und sich somit auch hinsichtlich $q_{d y n}\left(t_{e}\right)$ unterscheiden, wird die Ansteuerdauer entsprechend angepasst, um stets dieselbe Masse einzuspritzen.

\subsubsection{Druckverlaufsanalyse zur Bewertung der Verdunstungsneigung von SRE-Sprays}

Das mit einer Abtastrate von $100 \mathrm{kHz}$ zeitlich hoch aufgelöste Signal des Kammerdrucks weist einen charakteristischen Kurvenverlauf auf und ist beispielhaft in Abbildung 3.2 dargestellt. Der Druckverlauf ist gekennzeichnet durch einen kurzfristigen Druckabfall unter das Ausgangsniveau, unmittelbar nach dem Start der Einspritzung,

\footnotetext{
${ }^{1} \mathrm{Am}$ Vollmotor für $\lambda=1$ erforderliche Einspritzmasse bei $p_{S R}=1000$ mbar ist $m_{i n j} \approx 36 \mathrm{mg}$
} 
gefolgt von einer degressiven Annäherung an eine Grenzkurve für isotherme Verdunstung. Einen weiteren thermodynamischen Grenzfall stellt die adiabate Verdunstung dar. Bei beiden Grenzfällen handelt es sich um berechnete, theoretische Druckverläufe, die in der Praxis nicht auftreten können. Der Temperaturausgleich zwischen Kammervolumen und Umgebung kann weder unendlich schnell ablaufen (isotherm), noch kann er vollständig durch Wärmeisolierung unterbunden werden (adiabat). Dennoch spielen die beiden Grenzfälle bei der Bewertung der Injektorsprays anhand der Druckverlaufsanalyse eine entscheidende Rolle. Zunächst sei jedoch auf die physikalischen Ursachen

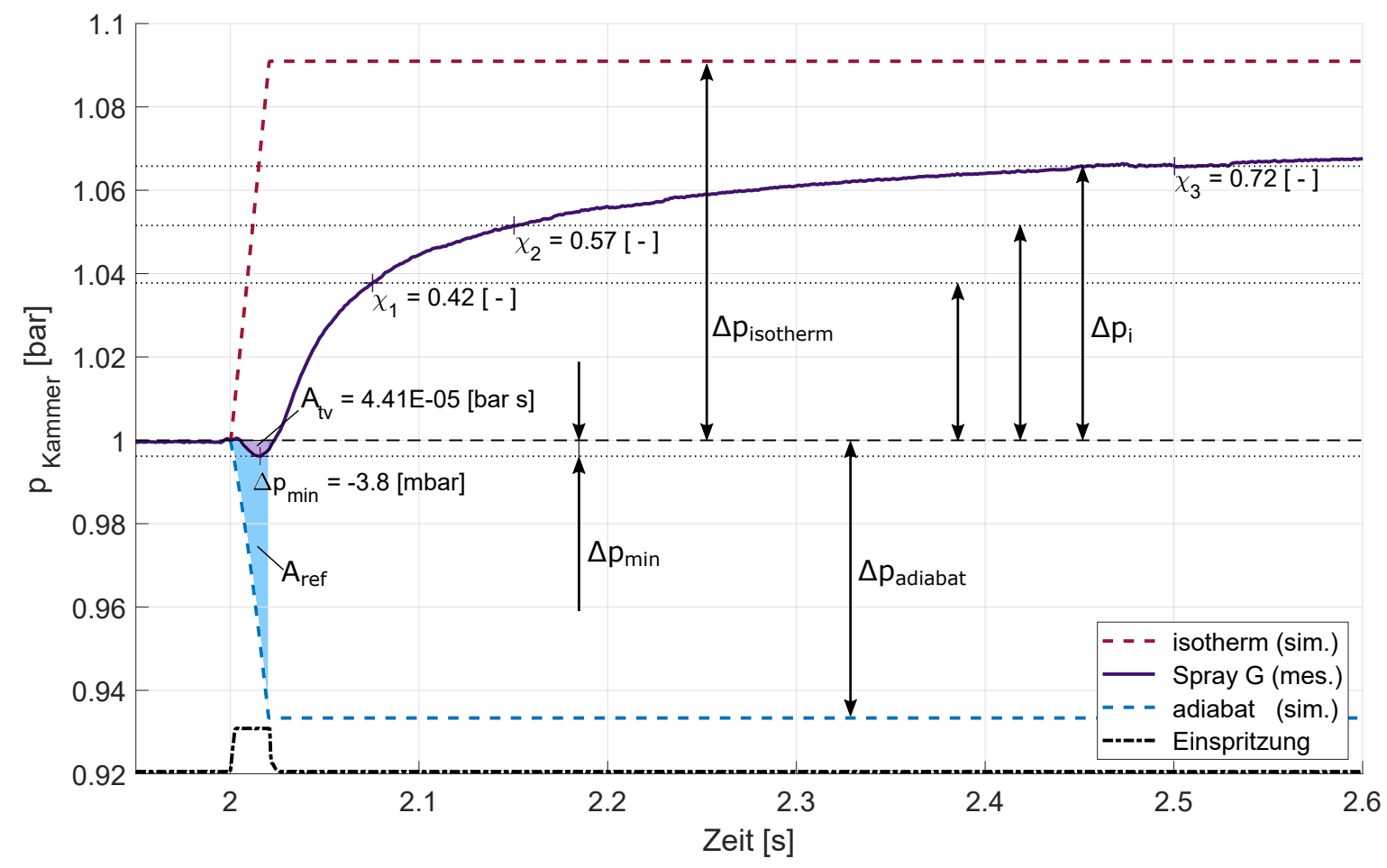

Abbildung 3.2.: Gemessener Kammerdruckverlauf für $T_{0}=93.5^{\circ} \mathrm{C}, p_{0}=1000 \mathrm{mbar}$ mit Injektor $\mathrm{G}$ sowie berechneten, thermodynamischen Grenzkurven

eingegangen, die für den gemessenen Druckverlauf bei konstantem Kammervolumen verantwortlich sind. Sobald die ersten Tropfen des Sprays die Injektorspitze verlassen, laufen mehrere konkurrierende Prozesse gleichzeitig ab, die in Tabelle 3.1 beschrieben sind.

Masseeintrag flüssiges n-Heptan

Temperaturabfall durch Verdunstungsenthalpie

Wärmeeintrag / Temperaturausgleich mit Umgebung

Erhöhung der Gasdichte durch Phasenübergang flüssig $\rightarrow$ gasförmig

$\begin{array}{ll}d m_{f l .} & p \uparrow \\ h_{V} & p \downarrow \\ d Q_{w} & p \uparrow \\ d \rho & p \uparrow\end{array}$

Tabelle 3.1.: Überlagerte Prozesse während der Spray-Verdunstung 
Durch die Einbringung von flüssigem n-Heptan reduziert sich das verbleibende Volumen für die Gasphase geringförmig. Der dadurch theoretisch resultierende Druckanstieg ist bei einer Einspritzmasse von $36.2 \mathrm{mg}$ mit weniger als 0.5 mbar jedoch sehr gering. Für den Phasenübergang von flüssig nach gasförmig muss die Verdunstungsenthalpie eines Stoffes aufgewendet werden. Bei der Tropfenverdunstung von Sprays geschieht dies durch Wärmetransport aus dem Inneren der Tropfen und aus der unmittelbar umgebenden Gasphase in die Phasengrenzschicht. Folglich fällt die Temperatur im Tropfen, wie auch die der umgebenden Gasphase, was wiederum zu einem Druckabfall im abgeschlossenen Volumen der Verdunstungskammer führt. Der somit entstandene Temperaturgradient zwischen Gasphase und der umgebenden Kammerwände sorgt für einen Wärmestrom von außen in die Verdunstungskammer. Mit steigender Kammertemperatur nimmt auch der Kammerdruck wieder zu. Zusätzlich sorgt der fortschreitende Phasenübergang des n-Heptans von flüssig nach gasförmig für eine höhere Dichte des Stickstoff-/n-Heptan-Gasgemisches und trägt dadurch ebenfalls zu einem Druckanstieg in der Kammer bei.

Der resultierende Druckverlauf ist also davon abhängig, wie stark die parallel ablaufenden Prozesse jeweils ausgeprägt sind. Der Grundgedanke der Verdunstungskammer liegt, wie eingangs beschrieben, darin, dass anhand des charakteristischen Druckverlaufs Rückschlüsse auf die Verdunstungsneigung eines Injektorsprays angestellt werden können. Aufgrund des deutlich größeren Oberflächen/Volumen-Verhältnisses wird angenommen, dass Tropfenverdunstung im Vergleich zu Wandfilmverdunstung deutlich schneller abläuft. Die Arbeiten von Gartung [36] und Schulz [109] untermauern diese Annahme (siehe Kapitel 2.2.4). Je nach Spray-Targeting ist die Spray-WandInteraktion unterschiedlich stark ausgeprägt. Es wird erwartet, dass sich diese Sprayunterschiede im Druckverlauf der Verdunstungskammer widerspiegeln. Zur quantitativen Beurteilung der Verdunstungsneigung verschiedener Sprays wird anhand des Druckverlaufs eine Bewertungszahl berechnet. Dafür ist der zeitliche Verlauf der thermodynamischen Grenzfälle der isothermen und adiabaten Verdunstung erforderlich. Im folgenden soll die Berechnung dieser beiden Grenzkurven erläutert werden.

Da neben dem Kammerdruck $p_{\text {Kammer }}(T)$ weitere Größen, wie der Sättigungsdampfdruck $p_{\text {sat }}(T)$, die Verdunstungsenthalpie $h_{v}(T)$ und die kalorischen Stoffdaten wie $c_{v}(T), c_{p}(T), h(T)$ und $u(T)$ von der Temperatur abhängig sind, ist eine iterative Berechnung in angemessenen Zeitschritten erforderlich. In Anlehnung an die Ausführungen von Pischinger et al. zur null-dimensionalen Modellierung eines Brennraums [85] (S.157ff) wird auf die Erhaltungssätze für Masse und Energie sowie auf die thermodynamische Zustandsgleichung idealer Gase zurückgegriffen.

$$
\begin{aligned}
d W+d Q+\sum d m_{i}\left(h_{i}+e_{a i}\right) & =d U+d E_{a} \\
p V & =m R T \\
\frac{d m}{d t} & =0
\end{aligned}
$$


Außerdem werden einige vereinfachende Annahmen getroffen.

- Die Verdunstungskammer wird als perfekt dicht betrachtet, sodass es keinen Leckagemassenstrom gibt.

- Ein null-dimensionaler Ansatz impliziert ein räumlich homogenes Verhalten aller Zustandsgrößen innerhalb der Kammer.

- Das Gas innerhalb der Verdunstungskammer verhält sich wie ein ideales Gas. Reibung innerhalb des Gases wird vernachlässigt.

- Die Volumenarbeit $d W$ ist aufgrund des isochoren Systems null.

- Die äußere Energie des Systems $E_{a}$, sowie die über die Systemgrenze fließende spezifische Energie $e_{a i}$, ist null.

Die Änderung der Masse $d m / d t$, welche ausschließlich durch die Einspritzung von nHeptan bestimmt ist, kann anhand der Injektorkennlinie abgeleitet werden. Für die Berechnung der Änderung der Zustandsgrößen Druck und Temperatur sind einige Umformulierungen von Gleichung 3.1 und 3.2 erforderlich. So gilt

$$
d U=d m u(T)+d u m
$$

wobei die Änderung der inneren Energie $d u$ für isochore Systeme mit

$$
d u=c_{v}(T) d T
$$

berechnet werden kann. Im Zusammenhang mit dem Wärmetransports $d Q$ sind zwei Wärmeströme relevant: Der Wärmeaustausch mit den Kammerwänden $Q_{w}$ und somit mit der Umgebung bzw. dem Konditionierungsmedium, sowie die Wärmemenge $Q_{v}$, die aufgrund der Verdunstungsenthalpie $h_{v}(T)$ benötigt wird. Folglich gilt:

$$
\begin{aligned}
d Q & =d Q_{w}+d Q_{v} \\
d Q & =\alpha A_{V K}\left(T_{a}-T_{i}\right)-d m h_{v}(T)
\end{aligned}
$$

Die Temperaturabhängigkeit von $h_{v}(T)$ für n-Heptan wird anhand einer Korrelation aus dem VDI-Wärmeatlas [117] berechnet. Unter Berücksichtigung der erwähnten Annahmen, sowie der beschriebenen Umformungen, kann der erste Hauptsatz der Thermodynamik in Anwendung auf die Verdunstungskammer wie folgt formuliert werden:

$$
\begin{aligned}
d Q+\sum d m_{i} h_{i} & =m c_{v} d T+d m u \\
d Q_{w}+d Q_{v} & =m c_{v} d T+d m(u-h)
\end{aligned}
$$


Als zweite Gleichung wird die thermodynamische Zustandsgleichung für ideale Gase in differentieller Form herangezogen und aufgrund von $p d V=0$ entsprechend vereinfacht:

$$
V d p=m R d T+R T d m+m T d R
$$

An dieser Stelle sei hervorgehoben, dass sich neben der Temperatur, der Masse und dem Druck auch die spezifische Gaskonstante im Zusammenhang mit der Gasgemischzusammensetzung ändert und daher für jeden Zeitschritt entsprechend dem aktuellen Massenverhältnis aus $N_{2} / C_{7} H_{16}$ berechnet werden muss. Für eine effiziente Berechnung der Zielgrößen $d p, d T$ und $d m$ in Matlab wird das Gleichungssystem bestehend aus Gleichung 3.9, Gleichung 3.10 und Gleichung 3.3 in der Form $A \cdot X=Y$ aufgestellt:

$$
\left[\begin{array}{ccc}
m c_{v} & 0 & u-h \\
-m R & V & -R T \\
0 & 0 & 1
\end{array}\right]\left[\begin{array}{c}
d T \\
d p \\
d m
\end{array}\right]=\left[\begin{array}{c}
d Q_{w}+d Q_{v} \\
m T d R \\
d m
\end{array}\right]
$$

Die Lösung für jeden Zeitschritt kann dann mittels $X=A^{-1} \cdot Y$ erfolgen, und liefert dadurch den theoretischen, zeitlichen Verlauf des Kammerdrucks, der Temperatur und der Einspritzmasse. Um schließlich die gesuchten Grenzkurven zu erhalten, wird der Wärmeübergangskoeffizient $\alpha$ entsprechend parametriert. Für eine idealisierte, adiabate Verdunstung wird der Wärmeübergangskoeffizient $\alpha$ zu null gesetzt. Ein solcher Druckverlauf einer theoretischen, adiabaten Verdunstung ist beispielhaft in Abbildung 3.2 als blaue, gestrichelte Kurve dargestellt. Für eine idealisierte, isotherme Verdunstung genügt bereits ein Wärmeübergangskoeffizient von $\alpha \geq 3.0\left[\mathrm{~W} / \mathrm{m}^{2} \mathrm{~K}\right]$. Das Resultat ist als rote, gestrichelte Kurve in Abbildung 3.2 erkennbar.

Neben der Lösung des Gleichungssystems 3.11, muss im Hintergrund für jeden Zeitschritt überprüft werden, ob der Partialdruck des n-Heptans unterhalb des temperaturabhängigen Sättigungsdampfdrucks $p_{\text {sat }}(T)$ liegt. Die Berechnung des Sättigungsdampfdrucks erfolgt anhand der Antoine-Gleichung 3.12 und wird mit den stoffspezifischen Parametern für n-Heptan aus der Datenbank des NIST [79] durchgeführt.

$$
\left.\log _{10}\left(p_{\text {sat }}\right)=A-(B / T+C)\right)
$$

So zeigt sich beispielsweise, dass bei einer Kammertemperatur von $37^{\circ} \mathrm{C}$ und adiabater Rechnung nur $12.5 \mathrm{mg}$ verdunsten können, da im Laufe einer adiabaten Verdunstung die Kammertemperatur stark fällt und somit auch der Sättigungsdampfdruck deutlich abnimmt.

Die Bewertungszahl $K_{D V A}$ zum direkten Vergleich verschiedener Injektorsprays hinsichtlich ihres Verdunstungsverhaltens berücksichtigt den minimalen Druck $p_{\text {min }}$, den Flächeninhalt $A_{t v}$ unterhalb des Initialdrucks $p_{0}$ und den Abstand $\Delta p_{i}$ zur theoretischen, isothermen Grenzkurve an drei Zeitpunkten $\left(t_{i}=75,150\right.$ und $\left.500 \mathrm{~ms}\right)$, und ist wie folgt definiert: 


$$
\begin{aligned}
K_{D V A} & =A_{t v} a+\Psi b+\left(\chi_{1}+\chi_{2}+\chi_{3}\right) c \\
A_{t v} & =\int_{t_{0}}^{t_{p=0}}\left(p-p_{0}\right)(-1) d t \\
\Psi & =\frac{\Delta p_{\text {min }}}{\Delta p_{\text {adiabat }}} \\
\chi_{i} & =\frac{\Delta p_{i}}{\Delta p_{\text {isotherm }}} \\
a & =\frac{1}{A_{\text {ref }}} ; \quad b=1 ; \quad c=1 / 3 \\
A_{\text {ref }} & =\int_{t_{0}}^{t_{e}} \Delta p_{\text {adiabat }} d t
\end{aligned}
$$

Die Bestandteile der einzelnen Terme sind in Abbildung 3.2 eingezeichnet. Eine große Bewertungszahl $K_{D V A}$ deutet auf eine hohe Verdunstungsneigung und somit auf eine gute Sprayauslegung für die vorliegende Ansaugkanalgeometrie hin. Die ersten beiden Terme der Bewertungszahl $\left(A_{t v}, \Psi\right)$ nehmen dann große Werte an, wenn ein starker initialer Druckabfall aufgrund von ausgeprägter, lang anhaltender Tropfenverdunstung und spätem Wandkontakt des Sprays vorliegt. Der dritte Term $\left(\chi_{i}\right)$ wird groß, sofern eine rasche Annäherung an die isotherme Druckgrenzkurve, also eine zügige Wandfilmverdunstung gegeben ist.

Um Überlagerungen mit den ersten beiden Termen zur Tropfenverdunstung zu vermeiden, und um sicher die nachgelagerte Wandfilmverdunstung zu bewerten, wurden die erwähnten Bewertungszeitpunkte $t_{i}$ des dritten Terms $\left(\chi_{i}\right)$ bewusst spät gewählt, trotz der Kenntnis, dass die am Vollmotor erforderlichen Verdunstungszeiten bei beispielsweise $\mathrm{N}=2000 \mathrm{~min}^{-1}$ mit einer Zyklusdauer von $60 \mathrm{~ms}$ deutlich kürzer sind. Die Ergebnisse zu Sprayvergleichen anhand der beschriebenen Bewertungszahl werden in Kapitel 4.1.1 vorgestellt. 


\subsection{Geschleppter Transparentmotor}

\subsubsection{Versuchsaufbau Transparentmotor}

Zur quantitativen Analyse von Kraftstoffwandfilmen im Brennraum werden optische Untersuchungen an einem Einzylindermotor mit transparenter Laufbuchse durchgeführt. Zu diesem Zweck wird der Zylinderkopf des am Vollmotorprüfstand verwendeten Forschungsmotors (siehe Tabelle 3.4) in leicht modifizierter Form auf dem Rumpf eines Transparentmotors installiert. Dazu wurde eine Adapterplatte konstruiert, welche zum einen die Brennraumdächer von Zylinder eins bis drei von unten verschließt und zum anderen über die Zylinderkopfschrauben die Montage mit dem Rumpf des Schleppaggregats ermöglicht. Somit kann das Brennraumdach von Zylinder vier über einem transparenten Glasring angeordnet werden. Ventiltrieb und Einlasskanäle von Zylinder eins bis drei sind stillgelegt. Der Rumpf des Schleppaggregats stammt von einem Hatz 1D60Z Dieselmotor und wurde in vorangegangenen Arbeiten aufgrund seiner Einfachheit und Robustheit gewählt. Die technischen Daten der in dieser Arbeit aufgebauten Kombination aus Zylinderkopf und Rumpfmotor sind in Tabelle 3.2 aufgeführt. Der Aufbau des Experiments ist in einer schematischen Darstellung in

\begin{tabular}{ll}
\hline Bohrung & $82 \mathrm{~mm}$ (wie Vollmotor) \\
Hub & $85 \mathrm{~mm}$ \\
Hubraum & $449 \mathrm{~cm}^{3}$ \\
Anzahl Ventile pro Zylinder & 4 \\
Auslass-NW schließt $(\mathrm{h}=1,0 \mathrm{~mm})$ & $-9{ }^{\circ} \mathrm{KW} \mathrm{n.} \mathrm{LW-OT}$ \\
Einlass-NW öffnet $(\mathrm{h}=1,0 \mathrm{~mm})$ & $-2^{\circ} \mathrm{KW} \mathrm{n.} \mathrm{LW-OT}$ \\
Einspritzung & $\mathrm{SRE}$ \\
Systemdruck & 4 bar \\
Ersatzkraftstoff & iso-Oktan \\
Ladungsbewegung & 2-stufige Tumbleklappe \\
\hline
\end{tabular}

Tabelle 3.2.: Technische Daten des Einzylinder-Transparentmotors

Abbildung 3.3 zu sehen. Da für die regelmäßige Reinigung des Glaszylinders (g) eine Anhebung des Zylinderkopfs notwendig ist, werden die Nockenwellen nicht mehr über die Steuerkette angetrieben. Stattdessen erfolgt der Antrieb von Außen über eine eigens für dieses Experiment konstruierte, schnell lösbare Verbindung (d), welche mit einem Riemen mit der Kurbelwelle verbunden ist. Die Öldruck-gesteuerte Phasenverstellung der Nockenwellen muss dadurch allerdings entfallen. Die Steuerzeiten werden daher manuell über die Klemmung der Antriebswellen eingestellt. Das Saugrohr (c) kann in der Originalform des Vollmotors verwendet werden. Darin ist zum einen das Einspritzventil (e) vom Typ Bosch EV14 als auch eine bewegliche Ladungsbewegungsklappe (f), welche eine Verstärkung der Tumbleströmung im Brennraum ermöglicht, integriert. Der Originalkolben des Vollmotors wurde so modifiziert, dass 
er auf eine Kolbenverlängerung (h) montiert werden kann, welche wiederum auf dem Kolben des Rumpfmotors befestigt ist. Für die optische Analyse von Kraftstoffwandfilmen kommen zwei Dimax-S High-Speed Kameras der Firma PCO zum Einsatz, welche zeitgleich Aufnahmen aus zwei Richtungen erzeugen. Die erste High-Speed Kamera (a) ist seitlich und angewinkelt angebracht, um ein Sichtfeld auf das Brennraumdach aufzuspannen. Der Bereich um die Einlassventile ist dadurch besonders gut sichtbar, aber auch Wandfilme, die unterhalb der Auslassventile an der Zylinderlaufbahn auftreten, können erfasst werden. Die zweite, waagerecht montierte, stirnseitige Kamera (b) er-
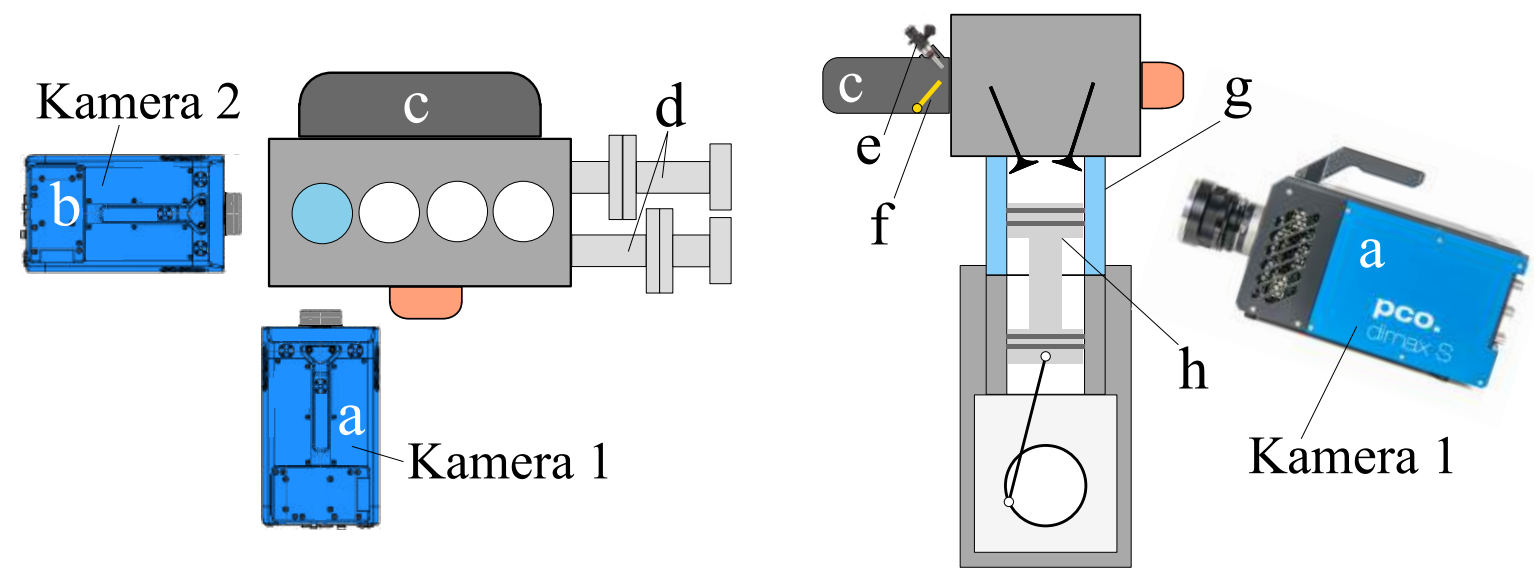

Abbildung 3.3.: Schematische Darstellung des optischen Setups am Transparentmotor von oben (linke Bildhälte) und von vorne (rechte Bildhälfte)

möglicht den Blick quer durch den Brennraum und insbesondere auf Wandfilme, welche seitlich des rechten (äußersten) Einlassventils an der Glaszylinderlaufbahn auftreten. An beiden Kameras kommen Nikon Objektive mit 35 mm Brennweite zum Einsatz. Für eine möglichst homogene Ausleuchtung von Brennraumdach und Brennraum werden vier LED-Lichtquellen verwendet, die außen um den Glaszylinder herum angeordnet sind. Zur Gleichstellung der Betriebsparameter mit dem Vollmotor für ausgewählte Betriebspunkte werden neben der Motordrehzahl weitere Messgrößen erfasst. Dazu gehören unter anderem der Saugrohrdruck, der Zylinderdruck, die Stellung der Ladungsbewegungsklappe, der Kraftstoffdruck und der Öldruck sowie Einspritzwinkel (EOI) und Einspritzdauer. Ein Foto des Versuchsaufbaus mit Transparentmotor und den beiden High-Speed Kameras ist in Abbildung 3.4 zu sehen.

Die Steuerung des Motors, die Messdatenerfassung sowie die synchrone Triggerung der High-Speed-Kameras erfolgt durch eine LabVIEW-basierte Software der Firma SoHaTex. Die Software ermöglicht außerdem eine sehr gute Reproduzierbarkeit der Versuche, indem Mess-Sequenzen vorab auf $0,1^{\circ} \mathrm{KW}$ genau programmiert werden können. Dadurch ist es möglich, sowohl die Einspritzung, als auch die Aufnahme eines einzelnen Bilds („Frame“) eines High-Speed-Videos zeitlich präzise an einer bestimmten Motorposition vorzugeben. Die Bildwiederholfrequenz („Frame-Rate“) der beiden 

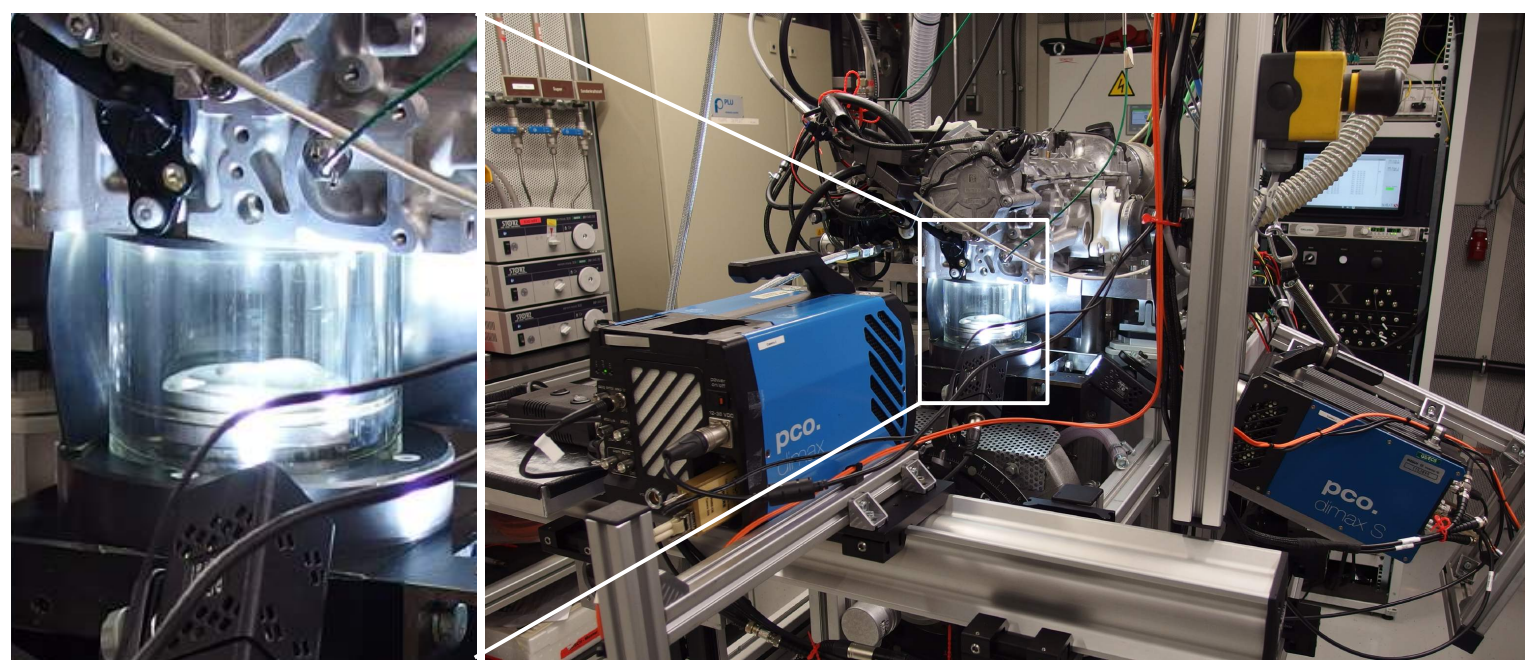

Abbildung 3.4.: Aufbau des Transparentmotors mit zwei PCO Dimax High-Speed Kameras

High-Speed-Kameras liegt bei $3000 \mathrm{fps}$, also einem Bildabstand von $4,0^{\circ} \mathrm{KW}$ bei der für alle Messungen eingestellten Motordrehzahl von $2000 \mathrm{~min}^{-1}$.

\subsubsection{Quantitative Analyse von Kraftstoffwandfilmen im Brennraum}

Das angestrebte Ziel des beschriebenen Versuchsaufbaus ist es, Kraftstoffwandfilme an potentiell problematischen Stellen innerhalb des Brennraums zu visualisieren und für Vergleiche zu quantifizieren. Insbesondere an Orten im Brennraum, die mittels endoskopischer Zugänge am Vollmotor nicht oder nur schlecht einsehbar sind. Anhand erster Analysen mittels High-Speed-Video Endoskopie am Vollmotor ergeben sich drei Regionen von besonderem Interesse, die in Tabelle 3.3 beschrieben sind. Die Ansichten der drei Perspektiven sind in der ersten Spalte (a) von Abbildung 3.5 dargestellt. Die Visualisierung einer Benetzung durch ein transparentes Medium wie Kraftstoff ist allerdings nicht trivial. Um den Kontrast zwischen trockenen und benetzten Oberflächen zu erhöhen, wird die Rauheit der untersuchten Oberflächen erhöht. Für die Einlassventile und das umgebende Brennraumdach stellt sich die Oberflächenbehandlung durch Sandstrahlen als zielführend heraus. Bei den Messungen zur Wandfilmanalyse an der Zylinderlaufbahn wird ein Klebestreifen aufgebracht, der durch Bestrahlung mit Glasperlen präpariert wurde. Es gilt sicherzustellen, dass der Bereich des Klebestreifens beim Betrieb des Motors nicht von den Kolbenringen überstrichen wird. Eine weitere Maßnahme zur Verbesserung der Bildqualität ist der Einsatz von iso-Oktan anstelle von Ottokraftstoff, da die hochsiedenden Anteile von Benzin mit der Zeit zu Ablagerungen führen, die den Kontrast verschlechtern. 


\begin{tabular}{|c|c|c|}
\hline Untersuchter Bereich / Region of Interest (ROI) & Kamera & Perspektive \\
\hline Einlassventile & Kamera 1 & $\begin{array}{l}\text {,1-V" glatte } \\
\text { Oberfläche }\end{array}$ \\
\hline $\begin{array}{l}\text { Zylinderlaufbahn („Liner“) unterhalb der } \\
\text { Auslassventile }\end{array}$ & Kamera 1 & $\begin{array}{l}\text { „1-L“ raue } \\
\text { Oberfläche }\end{array}$ \\
\hline $\begin{array}{l}\text { Zylinderlaufbahn („Liner“) seitlich, insbesondere } \\
\text { unmittelbar neben den Einlassventilen }\end{array}$ & Kamera 2 & $\begin{array}{l}\text {,2-L" raue } \\
\text { Oberfläche }\end{array}$ \\
\hline
\end{tabular}

Tabelle 3.3.: Am Transparentmotor untersuchte Bereiche (ROI) innerhalb des Brennraums

Die Methodik, wie anhand der Rohbilder aus den High-Speed Videos mittels eines Matlab-Skripts Wandfilmflächen ermittelt und bewertet werden, ist in den Spalten (a) bis (d) in Abbildung 3.5 und in einer vergrößerten Darstellung in Abbildung A.13 im Anhang visualisiert. Nachdem aus den Rohvideos die Bilder bei jeweils $78^{\circ} \mathrm{KW}$. Z-OT

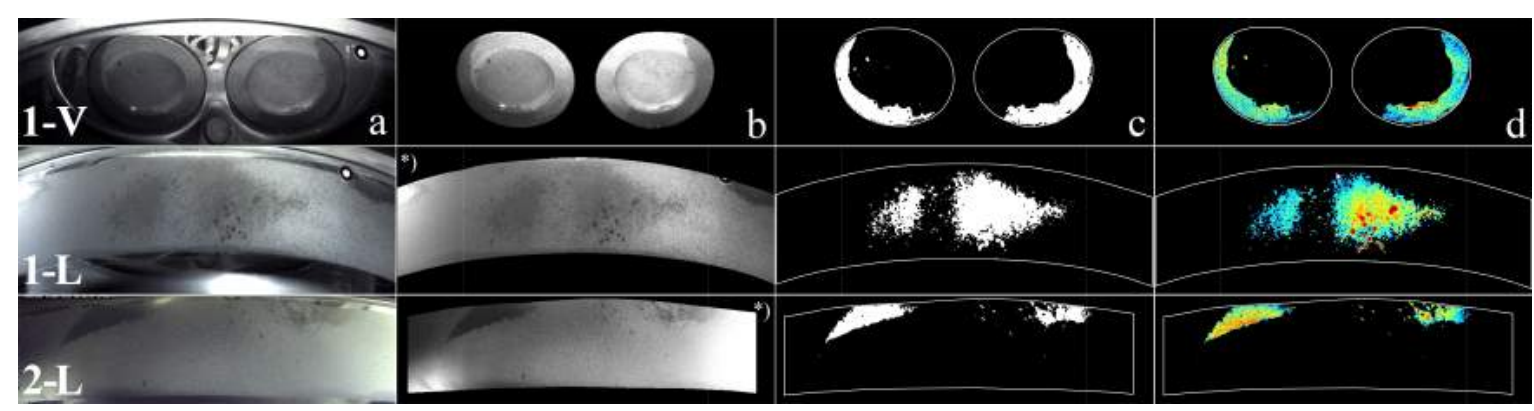

Abbildung 3.5.: Beispiele zur Methodik der Wandfilmquantifizierung am Transparentmotor für alle drei Perspektiven (1-V, 1-L, 2-L) in vier Schritten (a,b,c,d)

für die Perspektive $1-\mathrm{V}$ und bei $-62^{\circ} \mathrm{KWn}$. Z-OT für die Perspektiven 1-L und 2-L extrahiert wurden, erfolgt eine Korrektur der Farbtemperatur mittels Grauwertneutralisierung. Das Resultat dieser ersten Aufbereitung ist beispielhaft in Spalte (a) zu sehen. Im nächsten Schritt, dargestellt in Spalte (b), wird eine Maskierung vorgenommen, um die Bereiche von Interesse (ROI) zu isolieren, sowie eine Konvertierung der Farbaufnahme in ein schwarz-weiß Bild mit Grauabstufungen durchgeführt. Die Unterscheidung von benetzten und trockenen Pixeln innerhalb der ROI erfolgt im Schritt (c). Oberhalb eines Verdunklungsschwellwerts von $15 \%$ gegenüber einem Hintergrundbild gilt ein Pixel als benetzt. Das Hintergrundbild wird für jede Messung aus dem Mittelwert der ersten drei Zyklen ermittelt. Diese ersten drei Arbeitsspiele werden immer ohne Einspritzung erfasst. Durch den Vergleich mit diesem, unmittelbar vor der ersten Einspritzung erstellten, Hintergrundbild, können Fehler, durch zum Beispiel inhomogene Ausleuchtung oder Verschmutzungen, weitestgehend kompensiert werden. Durch die Anwendung von Sammelpixeln in Kombination mit den Matlab-Funktionen „,imdilute()“ und „imerode()“ können Einzelpixel-Effekte und Fehler durch Bewegungsunschärfe eliminiert werden. Da die geometrischen Flächeninhalte 
der ROI bekannt sind (kreisförmige Unterseite der Einlassventile, Zylindersegmente an der Laufbahn), können bereits an dieser Stelle Wandfilmflächen anhand der anteiligen Benetzung der ROI berechnet werden. In einem weiterführenden Schritt (d), kann der Einfluss der Wandfilmdicke mitberücksichtigt werden. Dazu erfolgt eine Gewichtung aller als benetzt markierten Pixel mittels der Intensität der Verdunklung. Anhand dieser Methodik ist zwar keine exakte Messung einer absoluten Wandfilmdicke möglich. Dennoch kann - optional - durch diese Gewichtung die gewonnene Bildinformation zur Wandfilmdicke in die Bewertung eines Wandfilms miteinbezogen werden.

Um belastbare Rückschlüsse auf die Auswirkungen der Injektorspray-Auslegung und Parametern wie Einspritztiming oder die Ladungsbewegung anstellen zu können, wird eine statistische Auswertung der detektierten Wandfilme von 10 Arbeitsspielen vorgenommen. Dabei wird ermittelt, wie häufig ein Pixel als benetzt erkannt wurde. Das Ergebnis dieser Auswertung ist in Abbildung 3.6 für alle drei Perspektiven beispielhaft dargestellt. Diese Analyse liefert einerseits Aufschluss darüber, an welchen Stellen innerhalb der ROI mit Kraftstoffwandfilmen zu rechnen ist, und anderseits, ob die erkannten Wandfilme statistisch relevant sind.

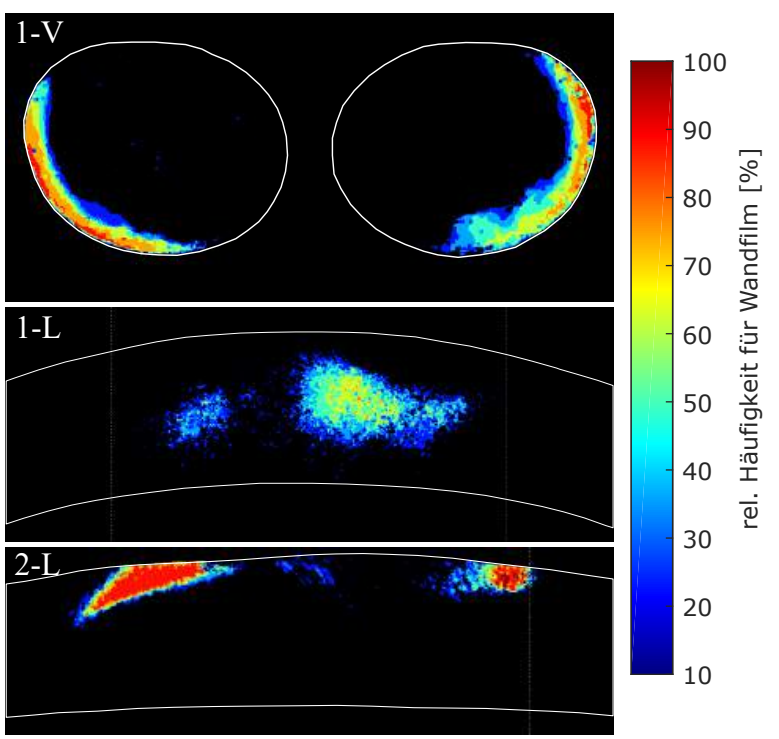

Abbildung 3.6.: Beispiele für Analysen hinsichtlich der Auftretenswahrscheinlichkeit von Kraftstoffwandfilm anhand statistischer Auswertung von 10 Zyklen

Eine weitere Methode zur Filmdickenabschätzung ist die Bewertung der Verdunstungszeit nach Abschaltung der Einspritzung bei weiterhin geschlepptem Motor. Da jedoch im Einlasskanal gespeicherter Kraftstoff nachfließen kann, ist diese Methode wenig aussagekräftig. 


\subsection{Vollmotor mit endoskopischen Zugängen}

\subsubsection{Versuchsträger und Optischer Aufbau}

Der Großteil der Untersuchungen dieser Arbeit wird an einem seriennahen Vollmotor durchgeführt, der durch Endoskopie-Bohrungen optisch zugänglich gemacht wurde. Dabei handelt es sich um ein Forschungsaggregat mit vier Zylindern, 2.01 Hubraum, Saugrohreinspritzung und Turbo-Aufladung. Die wesentlichen technischen Daten des Motors sind in Tabelle 3.4 dokumentiert. Insbesondere die Freiheitsgrade im Bereich

\begin{tabular}{ll}
\hline Bohrung & $82 \mathrm{~mm}$ \\
Hub & $94 \mathrm{~mm}$ \\
Hubraum & $1990 \mathrm{~cm}^{3}$ \\
Verdichtungsverhältnis $\epsilon$ & 11.5 \\
Anzahl Ventile / Zylinder & $4 / 4$ \\
Einspritzsystem & $\mathrm{SRE}$ \\
Systemdruck & $3-10 \mathrm{bar}$ \\
Kraftstoff & Super 95 E10 \\
Aufladung & Turbolader mit Wastegate \\
Ladungsbewegung & 2-stufige Tumbleklappe \\
Auslass- / Einlassventiltrieb & Phasenverstellung \\
Optische Zugänge & Endoskope: Brennraum \& Saugrohr \\
\hline
\end{tabular}

Tabelle 3.4.: Technische Daten des Vollmotors

der Ventilsteuerzeiten durch die Möglichkeit der Phasenverstellung auf der Einlass- und Auslassseite, sowie die verstellbare Ladungsbewegungsklappe im Saugrohr sind für die vorliegende Arbeit von Interesse, da hiermit Wechselwirkungen auf die Gemischbildung in Saugrohr und Einlasskanal untersucht werden können.

Der Zylinderkopf wurde im Vorfeld bearbeitet, um Drucksensoren (Indizierquarze) integrieren zu können. Im Brennraum aller vier Zylinder kommen zur Hochdruckindizierung wassergekühlte, piezoelektrische Druckquarze vom Typ Kistler 6041A zum Einsatz $\left(p_{C y l}\right.$ in Abbildung 3.7). Im Einlasskanal von Zylinder drei und vier werden piezoresistive Quarze vom Typ Kistler 4005B $\left(p_{i n}\right)$ und im Auslasskanal derselben Zylinder piezoresistive Quarze vom Typ 4049A $\left(p_{e x}\right)$ verwendet. Dadurch können zwar nicht alle Zylinder mit einer Niederdruckindizierung abgedeckt werden, eine Redundanz zur Ausfallsicherheit ist aber gegeben. Die Niederdruckindizierung ist vor allem für die in Kapitel 4.1.2 vorgestellten Ladungswechselrechnungen erforderlich. Die Einspritzventile sind so in das Saugrohr integriert, dass die Injektorspitzen stromabwärts unmittelbar vor der Dichtkante zum Zylinderkopf in die Ansaugströmung ragen (siehe Abbildung 3.8). Dadurch wird, trotz der Montage im Saugrohr, ein möglichst geringer Abstand zu den Einlassventilen von etwa $90 \mathrm{~mm}$ ermöglicht. Unmittelbar unterhalb 


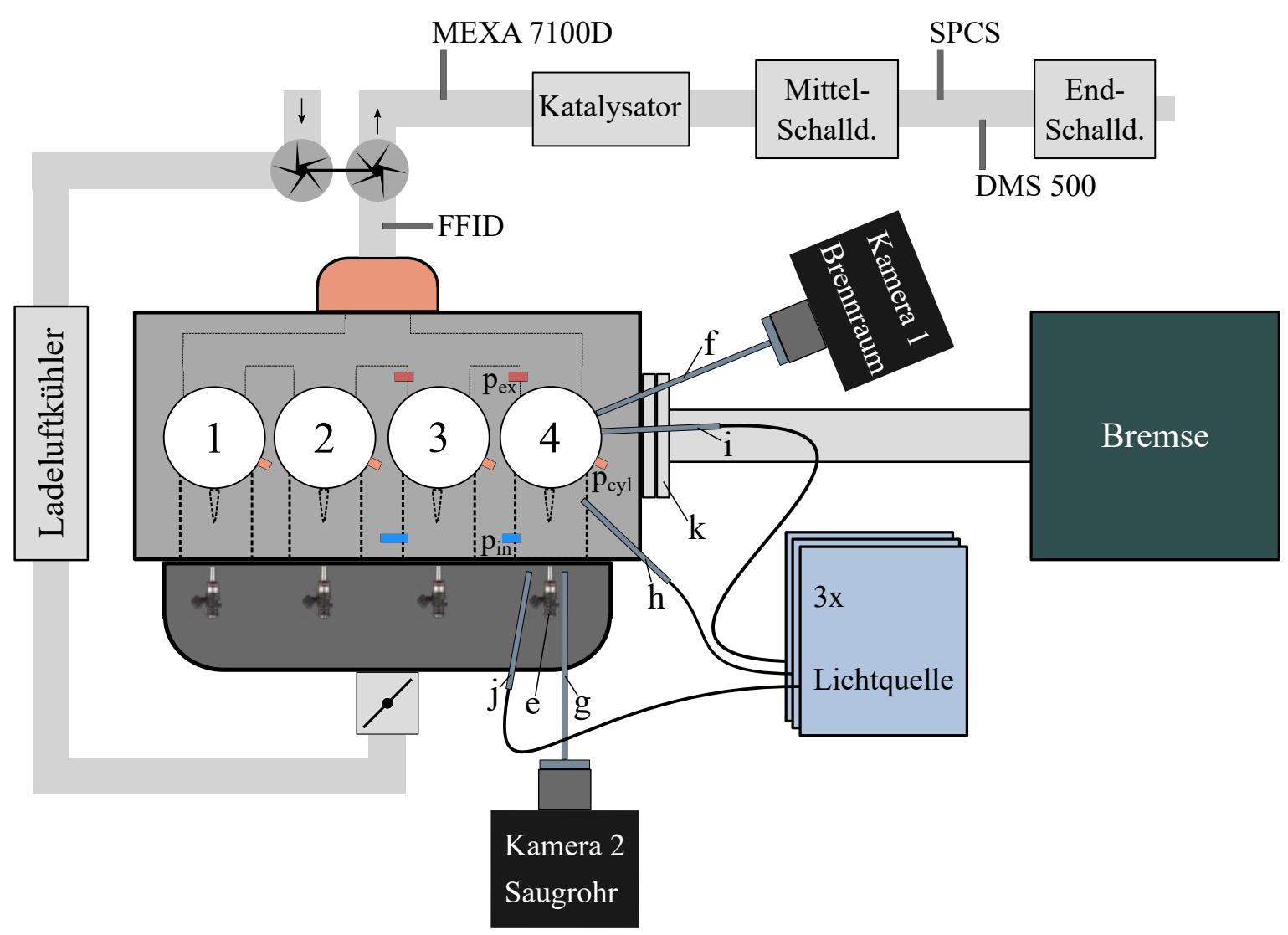

Abbildung 3.7.: Versuchsaufbau am Vollmotorprüfstand

des Abspritzpunktes befindet sich die Ladungsbewegungsklappe (LBK), sodass diese bei konventionell gestalteten Kraftstoffsprays selbst bei angestellter LBK nicht direkt vom Kraftstoff getroffen wird.

Weiterhin wurde der Zylinderkopf dahingehend bearbeitet, dass endoskopische Zugänge in den Brennraum von Zylinder vier für Lichteinkopplung (i) und Bilderfassung (f) geschaffen wurden. Ein weiterer Zugang (h) ermöglicht die Beleuchtung des Einlasskanals. Außerdem wurde das Saugrohr modifiziert, um ebenfalls einen Adapter zur Lichteinkopplung (j) und ein Endoskop zur Bilderfassung (g) zu integrieren. Eine detailliertere Ansicht dieser optischen Zugänge ist in Abbildung 3.8 dargestellt. Aufgrund der Anordnung der optischen Komponenten ist die Perspektive des BrennraumEndoskops leicht zur Seite der Einlassventile (a) geneigt, während die Beleuchtung zur Brennraummitte hin und somit zur Zündkerze (c) gerichtet ist. Am Vollmotor kommen zwei High-Speed Kameras der Firma Photron vom Typ Fastcam Mini AX-200 zum Einsatz. Bei Kamera 1, für den Blick in den Brennraum, handelt es sich um eine Farbkamera. Dies ist insbesondere bei der Unterscheidung von rötlichen Diffusionsflammen von tendenziell bläulichen Flammen der gut homogenisierten, vorgemischten Verbrennungen hilfreich. Kamera 2, welche die Vorgänge im Saugrohr und Einlasskanal dokumentiert, ist eine schwarz-weiß Kamera. Zur Analyse der Einspritzung und 

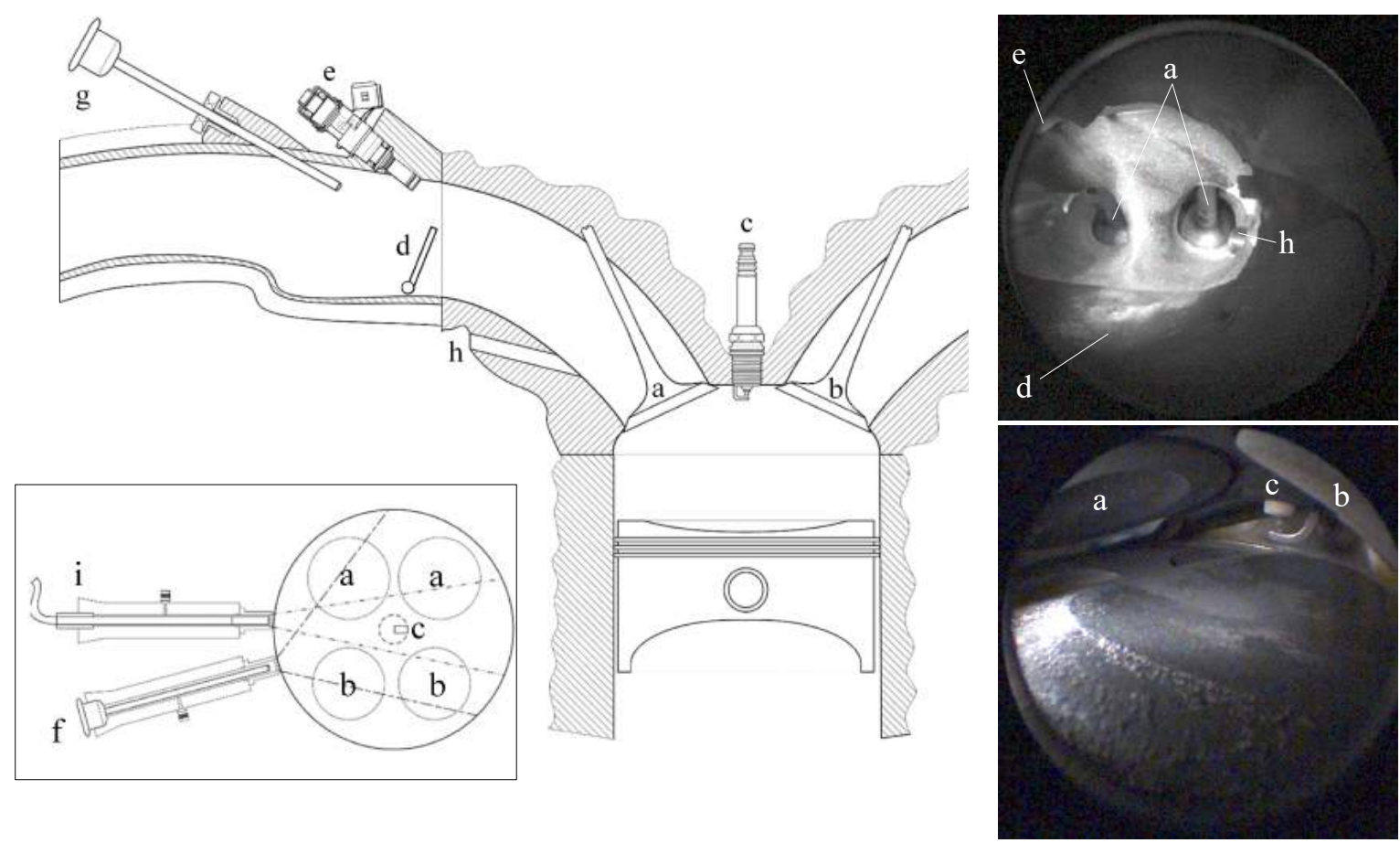

Abbildung 3.8.: Schema und Perspektiven der optischen Zugänge zur HSVE

der Wechselwirkung von Kraftstoffspray und Luftströmung ist keine Farbinformation erforderlich. Bei den verwendeten Lichtquellen handelt es sich um Kaltlichtquellen der Firma Storz vom Typ Xenon Nova 300.

Für Versuche zu Kaltstart und Katalysator-Heizen ist ein Fast-Flame-IonisationDetector (FFID) vorgesehen, dessen Entnahmesonde für eine möglichst kurze Gaslaufzeit direkt stromabwärts des Auslasskrümmers platziert ist (siehe Abbildung 3.7). Eine Kupplung (k), welche die Kurbelwelle von der Abtriebswelle zur Bremse trennen kann und elektro-pneumatisch betätigt wird, ist zusätzlich für Kaltstartversuche installiert. Zwischen der Turbine des Turboladers und dem Katalysator befindet sich die Entnahmesonde für die Abgasanalyseanlage vom Typ Horiba Mexa 7100D. Sie erfasst die Konzentration der gasförmigen Schadstoffe $\left(\mathrm{HC}, \mathrm{CO}, \mathrm{NO}_{x}\right)$ sowie des Restsauerstoffs $\mathrm{O}_{2}$ und des $\mathrm{CO}_{2}$. Die Abgasentnahmesonde für den Partikelzähler SPCS2100 der Firma Horiba befindet sich stromaufwärts des Endschalldämpfers. Die Entnahmesonde des Partikelanalysators DMS500 der Firma Cambustion befindet sich in unmittelbarer Nähe dazu. Beide Entnahmestellen wurden bewusst verhältnismäßig weit stromabwärts platziert, um ähnliche Bedingungen zu sogenannten „Tail-pipe“ Messungen zu schaffen; also einer Messung am Endrohr der Abgasanlage, wie dies beispielsweise bei PEMS-Messungen auf der Straße praktiziert wird. Eine genauere Erläuterung der Messprinzipien, sowie der Vor- und Nachteile der beiden Partikelzähler, erfolgt in Kapitel 3.3 .3 .

Die Vollmotorversuche erfolgen auf einem typischen, dem Stand der Technik entsprechenden Motorprüfstand mit elektrischer Bremse. Die Konditionierung für Motorkühl- 
wasser und Motoröl ist so ausgelegt, dass auch ein dauerhaft zwangsgekühlter Motorbetrieb möglich ist. Des Weiteren wird eine Vielzahl an Drücken und Temperaturen in Luftpfad, Kraftstoffpfad, Öl- und Wasserkreislauf erfasst. Durch die automatisierte Überwachung von parametrierbaren Grenzwerten, sowie durch programmierbare Messabläufe und Regler mittels „RBAS“ (Robert Bosch Automatisierungs-Software), können umfangreiche Messungen auch unbemannt über Nacht erfolgen. Versuche mit Endoskopie und High-Speed Kameras sind aufgrund der sensiblen Messtechnik jedoch nur bemannt zu fahren.

\subsubsection{Methodik zur Lokalisierung von Partikel-Quellen}

Neben der Analyse verschiedener Einflussparameter auf die Partikelemissionen bei Saugrohreinspritzung stellt die Lokalisierung der Partikelentstehungsorte innerhalb des Brennraums einen wesentlichen Bestandteil dieser Arbeit dar. Bei genauer Kenntnis der Partikelquellen im Brennraum fällt es leichter, erforderliche Maßnahmen zur Reduktion der Partikelbildung abzuleiten.

Wie in Abschnitt 2.3 ausführlich erläutert, stellen auch in Ottomotoren diffusionskontrollierte Verbrennungen die physikalische Ursache für die Partikelentstehung dar. Diffusionsflammen sind anhand ihrer gelblichen bis rötlichen Farbe gut von der eher bläulichen (bis ultra-violetten) Flamme einer stöchiometrischen, gut homogenisierten Verbrennung unterscheidbar [105]. Der charakteristische Farbton des sogenannten Rußleuchtens kann mit dem Planckschen Strahlungsgesetz für schwarze Körper erklärt werden. Dies trifft zu, da Ruß, aufgrund seiner Emissivität $E$ nahe 1, einen fast perfekten schwarzen Strahler darstellt. Die maximale, spektrale Energiedichte des Rußleuchtens liegt für typische Rußtemperaturen während einer ottomotorischen Verbrennung im Wellenlängenbereich der Infrarotstrahlung. Die emittierte Strahlung im angrenzenden Spektralbereich des sichtbaren Lichts, also im Bereich von rotem und gelbem Licht, ist dennoch energiereich genug, um von Kamera 1 im Brennraum erfasst zu werden. Für detailliertere Informationen zur Inkandeszenz von Rußpartikeln, sei an dieser Stelle auf die Literatur verwiesen [115, 127]. Die bläuliche Strahlung einer vorgemischten Verbrennung entsteht demgegenüber durch Chemielumineszenz, welche bei der Oxidation von organischen Verbindungen mit Sauerstoff auftreten kann [11].

Zur statistischen Auswertung der Entstehungsorte der Diffusionsflammen anhand der High-Speed Videos wurde ein Matlab-Skript erstellt. Die Vorgehensweise bei der Analyse der Bilder ist vergleichbar mit der am Transparentmotor angewandten Methode (siehe Abschnitt 3.2.2). An einer geeigneten Motorposition (z.B.: bei $15^{\circ} \mathrm{KW}$. Z-OT) werden die Bilder aller aufgezeichneten Arbeitsspiele aus dem Video der Brennraumkamera extrahiert. Die rötlichen Diffusionsflammen sind zwar mit dem Auge gut anhand ihrer Farbe zu erkennen, eine automatisierte Detektion gelingt allerdings am besten mittels einer Gradientenerkennung innerhalb eines in Graustufen überführten Bildes 
(Matlab-Funktion „edge()“). Insofern ist die Helligkeit der Diffusionsflamme ausschlaggebend für die Detektion. Auch an dieser Stelle können die Matlab-Funktionen „,imdilute()" und " "imerode()" Artefakten und Einzelpixeleffekten erfolgreich entgegenwirken. Durch Zählung der erkannten Diffusionsflammen für jedes Pixel, kann - bezüglich der Anzahl an analysierten Verbrennungszyklen - eine Häufigkeit für das Auftreten einer Diffusionsflamme an jedem sichtbaren Ort im Brennraum berechnet werden. Abbildung 3.9 zeigt ein Beispiel für die beschriebene Methode. In der linken Bildhälfte (a) ist eine Diffusionsflamme in der Nähe der Einlassventile erkennbar. Dass dies nicht nur

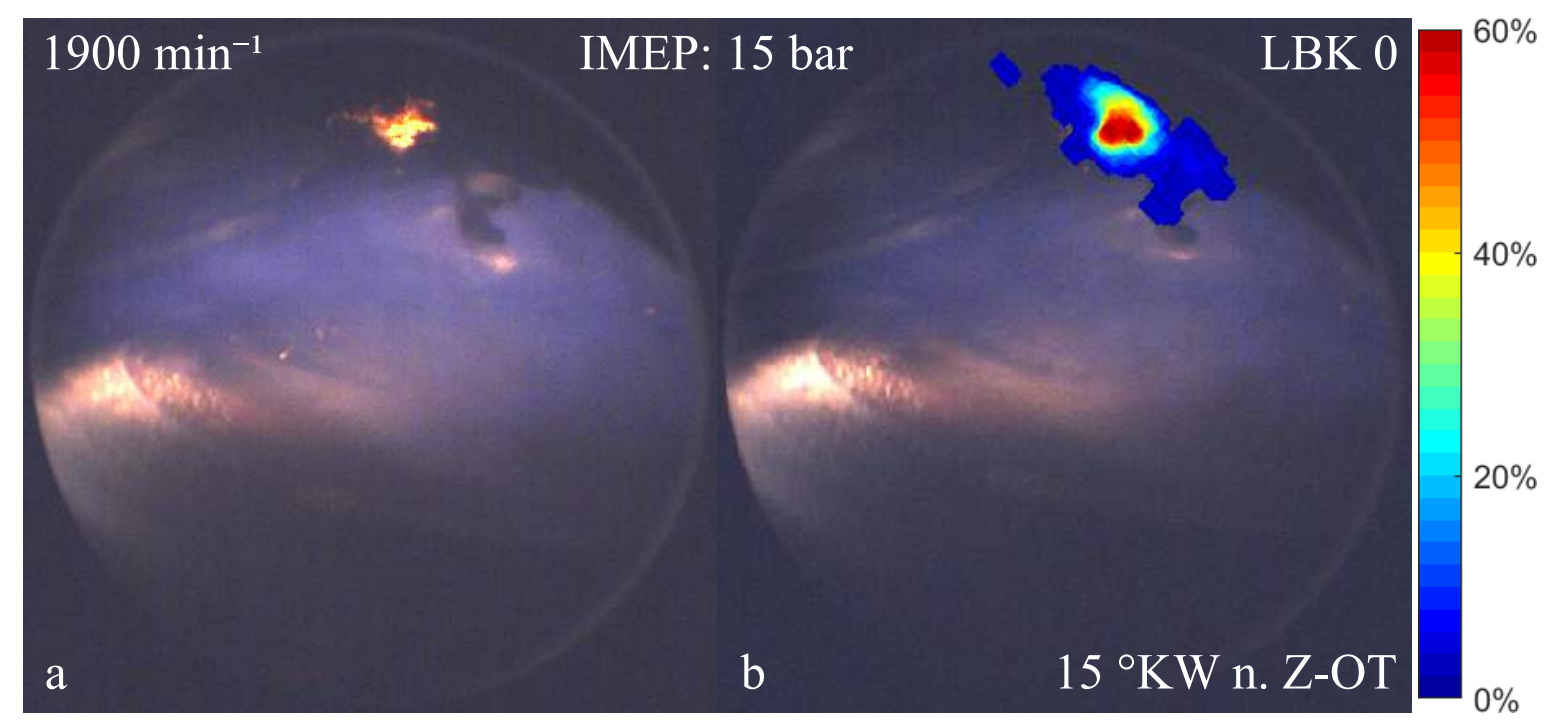

Abbildung 3.9.: Beispiel zur statistischen Analyse von Partikelquellen im Brennraum:

(a) rötliche Diffusionsflamme einer einzelnen Verbrennung links;

(b) Häufigkeit für erkannte Diffusionsflammen von 40 Zyklen rechts

für ein einziges Arbeitsspiel gilt, sondern statistisch abgesichert werden kann, zeigt die Häufigkeit der Diffusionsflammen an dieser Stelle in der rechten Bildhälfte (b). Das gezeigte Beispiel stammt aus einer Messung mit dem stationären Betriebspunkt mit $\mathrm{N}=1900 \mathrm{~min}^{-1}$, IMEP $=15$ bar und deaktivierter Ladungsbewegungsklappe $(\mathrm{LBK})$.

\subsubsection{Partikelmesstechnik}

Da die Partikelanzahlemission von zentraler Bedeutung für die vorliegende Arbeit ist, soll im Folgenden die am Vollmotorprüfstand dafür eingesetzte Messtechnik beschrieben werden. Bei ausnahmslos allen Versuchen war zur Messung der Partikelanzahlkonzentration im Abgas $\left(P N\left[\# / \mathrm{cm}^{3}\right]\right)$ stets ein Partikelzähler der Firma Horiba vom Typ SPCS2100 installiert. Als Sensor zur Partikelzählung ist in diesem Messgerät ein Kondensationspartikelzähler vom Typ TSI CPC-100 verbaut. Die zu zählenden Partikel werden in eine mit Butanoldampf gesättigte Kammer geleitet und fungieren dort als Kondensationskeime. Die dadurch entstandenen Tröpfchen sind größer 
als die ursprünglichen Partikel und führen zur Streuung eines Laserstrahls. Dies wird mit einem Photodetektor erfasst. Sowohl die gezielte Einschränkung auf Partikel, die nicht kleiner sind als $23 \mathrm{~nm}$, als auch die angewandte Technik zur Verdünnung des Abgases und zur Entfernung von flüchtigen Partikel mittels eines „Volatile Particle Remover" (VPR) entspricht den Vorgaben des Gesetzgebers sowie den Empfehlungen durch das „Particle Measurement Programme“ (PMP) der UNECE. Da der gesetzlich vorgeschriebene, untere Grenzdurchmesser von $23 \mathrm{~nm}$ im Sinne eines fixen "Cut-off" Punktes messtechnisch nicht darstellbar ist, wird der Partikelzähler so kalibriert, dass sich für Partikeldurchmesser von $23 \mathrm{~nm}$ eine Zähleffizienz von $50 \%( \pm 12 \%)$ einstellt und für Partikeldurchmesser mit $41 \mathrm{~nm}$ von mindestens $90 \%$. Es handelt sich also vielmehr um eine „Cut-off“ Kurve. Das Messgerät zeichnet sich neben der Tauglichkeit für gesetzeskonforme PN-Messungen dadurch aus, dass es durch die eingesetzte Verdampfungsröhre („Evaporation tube“), die auf $350^{\circ} \mathrm{C}$ beheizt ist, flüchtige Partikel effizient beseitigt.

Bei dem für ausgewählte Versuche parallel installierten DMS500 ist dies in Frage zu stellen. Durch die deutlich geringere Verdünnung des Abgases, sowie durch das Fehlen einer Verdampfungsröhre oder einer alternativen Technik zur Entfernung flüchtiger Partikeln, ist zu erwarten, dass auch flüssige Partikel gezählt werden. Dennoch liegen die Stärken dieses Messgerätes auf der Hand. Zum einen ist die gegenüber dem SPCS2100 deutlich kürzere Ansprechzeit für Messungen bei dynamischem Motorbetrieb vorteilhaft, zum anderen liefert das DMS500 durch die Größenklassierung der gezählten Partikel eine weitere wertvolle Information: das Größenspektrum der emittierten Partikel. Das Messprinzip des DMS500 ist grundsätzlich verschieden von dem des SPCS2100. Jedes Partikel wird mit einer positiven, elektrischen Ladung versehen, die zu dessen Oberfläche proportional ist. Die geladenen Partikel werden in einen Klassierungsabschnitt geleitet, in dem ein starkes, radiales, elektrisches Feld vorherrscht. Aufgrund des Feldes driften die Partikel durch eine Hüllströmung nach außen, in Richtung der Detektor-Ringe, die als Elektrometer fungieren. In Abhängigkeit von der elektrischen Mobilität des Partikels, also dem Verhältnis aus Strömungswiderstandskraft und elektrischer Feldkraft, trifft es auf Detektorringe bereits am Eingang des Größenklassierers oder auf Ringe, die weiter stromabwärts platziert sind. Dadurch wird gleichzeitiges Zählen und Klassieren nach Partikelgröße ermöglicht.

Eine Gegenüberstellung der beiden eingesetzten Partikelzähler ist in Tabelle A.2 im Anhang zu finden. Für tiefergehende Informationen zur Partikelmesstechnik sei auf den Abschlussbericht des FVV-Vorhabens Nr. 1073 „PN-Messverfahren“ 663] verwiesen. 


\subsection{Injektor-Varianten}

Die im Laufe der vorliegenden Arbeit ausgelegten und verwendeten Injektor-Muster sind in Tabelle 3.5 dokumentiert. Die Definition der beschriebenen Spray-Winkel ist in Abbildung 2.2b beschrieben. Injektoren mit einem (einzelnen) Kegelstrahl sind in Reihe 3 als C-Typ markiert. Injektoren mit der Sprayform „Zweistrahl“ werden als E-Typ bezeichnet. Bei der Auslegung dieser Injektor-Muster wurden die Spray-Parameter $\alpha_{50^{-}}$

\begin{tabular}{|c|c|c|c|c|c|c|c|c|c|}
\hline Injektor & $\mathbf{A}$ & B & $\mathrm{C}$ & $\mathrm{D}$ & $\mathbf{E}$ & $\mathbf{F}$ & G & $\mathbf{H}$ & $\mathbf{I}$ \\
\hline Variation & Basis & Basis & $\alpha_{50}$ & $\alpha_{50}$ & $\beta_{80}$ & $\beta_{80}$ & $\beta \& \mathrm{SMD}$ & SMD & SMD \\
\hline Тур [-] & $\mathrm{E}$ & E & $\mathrm{E}$ & $\mathrm{E}$ & $\mathrm{E}$ & $\mathrm{E}$ & $\mathrm{E}$ & $\mathrm{E}$ & $\mathrm{C}$ \\
\hline $\boldsymbol{Q}_{\text {stat }}[\mathrm{g} / \mathrm{min}]$ & 106 & 106 & 106 & 106 & 106 & 116 & 110 & 122 & 75 \\
\hline$\alpha_{50}\left[{ }^{\circ}\right]$ & 20,4 & 22,4 & 27,0 & 33,3 & 27,4 & 35,0 & 36,0 & 39,5 & 28 \\
\hline$\beta_{80}$ & 8,8 & 11,3 & 10,4 & 10,4 & 13,8 & 25,0 & 30,0 & 23,3 & - \\
\hline$\gamma$ & 11,4 & 7,4 & 4,8 & 4,9 & 3,5 & 0,0 & 0,0 & 0,0 & 0,0 \\
\hline $\boldsymbol{S} \boldsymbol{M D}[\mu m]$ & 85,7 & 70,0 & 67,8 & 68,1 & 55,9 & 57,5 & 49,6 & 52,9 & 40,0 \\
\hline
\end{tabular}

Tabelle 3.5.: Kenndaten der untersuchten Injektor-Spray Varianten bei $p_{K r}=3.0 \mathrm{bar}$

Winkel, $\beta_{80}$-Winkel und der mittlere Sauter-Durchmesser der Tropfen (SMD) gezielt variiert (siehe Reihe 2 „Variation“ in Tabelle 3.5). Eine Übersicht mit grafischen Darstellungen der beschriebenen Spray-Auslegungen ist in Abbildung A.14 im Anhang beigefügt. Weitere, speziell für eine Twin-Konfiguration ausgelegte, Injektor-Muster $\mathrm{J}, \mathrm{K}$ und $\mathrm{L}$ werden in Kapitel 4.2.7 vorgestellt und in Abbildung 4.30 visualisiert. Ausgewählte Injektor-Muster kommen an allen drei Versuchsträgern, der Verdunstungskammer, am geschleppten Transparentmotor und am gefeuerten Vollmotor zum Einsatz, um ein umfassendes Verständnis zu den Anforderungen an die Sprayauslegung hinsichtlich der Reduktion von PN-Emissionen zu erlangen.

An dieser Stelle sei in Kürze auf die angewandten Messmethoden zur Bestimmung der Spray-Eigenschaften eingegangen. Die Spray-Winkel werden anhand einer sogenannten Matrix-Messung ermittelt. Nach einer definierten Zahl von Einspritzungen können, anhand der Füllstände in den Zellen der Matrix, die Spray-Winkel berechnet werden. Die Tropfengrößenbestimmung erfolgt mit dem Messsystem Aerotrac LDSA1500A der Firma MicrotracBEL, bei welchem ein Messprinzip auf Basis der Laserbeugung, genauer der Fraunhofer-Beugung, an den Tropfen eines Sprays angewandt wird. Die Tropfen führen hierbei zu einer Streuung im Strahlengang, wodurch ein Beugungsbild erzeugt werden kann, anhand dessen auf die Tropfengröße zurückgerechnet wird. Sehr dichte Sprays stellen aufgrund von Mehrfachbeugung hierbei eine Herausforderung dar. Dieses Verfahren und die Matrix-Messung sind in der empfohlenen Vorgehensweise von Hung et. al [51] sowie im SAE Standard J2715 [99] zur Vermessung und Charakterisierung von Sprays für Ottokraftstoff-Injektoren beschrieben. 


\section{Ergebnisse}

\subsection{Grundlegende Untersuchungen der Gemischbildung im Einlasskanal}

Der Einlasskanal ist für die Saugrohreinspritzung von besonderer Bedeutung, da hier ein wesentlicher Teil der Gemischbildung stattfindet. Daher wird im folgenden Kapitel 4.1.1 zunächst die Gemischbildung anhand von Versuchen an einer Verdunstungskammer in Form eines Einlasskanals untersucht und im Anschluss in Kapitel 4.1.2 der Einfluss des Ladungswechsels auf die Gemischbildung analysiert.

\subsubsection{Bewertung von SRE-Injektorsprays anhand ihres Verdunstungsverhaltens}

\section{Einfluss des Saugrohrdrucks}

Zur isolierten Betrachtung des Einflusses des Saugrohrdrucks bzw. des Kammerdrucks $p_{0}$ zu Beginn der Messung auf die Gemischbildung wird zunächst die eingespritzte Kraftstoffmasse von $m_{i n j}=36 \mathrm{mg}$ n-Heptan konstant gehalten. Da sich die Druckdifferenz über das Einspritzventil mit dem Kammerdruck ändert, muss die Ansteuerdauer $t_{e}$ anhand der Bernoulli-Gleichung 1 angepasst werden, um stets dieselbe Masse einzuspritzen. In Abbildung 4.1 sind die zeitlichen Verläufe der Druckänderung $\Delta p_{\text {Kammer }}$ und die Kammertemperatur bei einer Initialtemperatur von $T_{0}=93^{\circ} \mathrm{C}$ dargestellt. Beim Kammerdruck von $p_{0}=300$ mbar ist eine deutlich schnellere Annäherung an die isotherme Grenzkurve zu beobachten. Dies deutet auf eine raschere Verdunstung als bei $p_{0}=1300$ mbar und 1000 mbar hin und deckt sich mit der Erwartung. Für die beiden höheren Kammerdrücke $p_{0}$ ist zwar unmittelbar nach der Einspritzung, anhand des deutlichen Druckabfalls, eine ausgeprägte Tropfenverdunstung erkennbar, die anschließende Wandfilmverdunstung ist aber langsamer als bei geringem Kammerdruck. Letzteres ist im Hinblick auf Kraftstoffwandfilme als Partikelentstehungsquelle als kritisch zu bewerten.

Hervorzuheben ist, dass trotz der günstigen Verdunstungsbedingungen bei geringem Initialdruck von $p_{0}=300$ mbar und warmer Kammer kein Druckabfall infolge des

\footnotetext{
${ }^{1}$ Vereinfachte Gleichung nach Bernoulli: $v_{T r} \approx \varphi \sqrt{\frac{2}{\rho}\left(p_{K r}-p_{K a m m e r}\right)}$ mit Durchflussbeiwert $\varphi<1$
} 
Temperaturabfalls durch die Verdunstungsenthalpie zu verzeichnen ist. Eine mögliche Erklärung für dieses Phänomen ist der bei diesen Bedingungen „überhitzte“ Kraftstoff, welcher im Injektor dieselbe Temperatur wie die Kammer von $T_{0}=93^{\circ} \mathrm{C}$ annimmt. Dadurch kommt es bereits im Spritzloch zum Sieden des Kraftstoffes, dem sogenannten „Flash Boiling ‘2 und somit zu einer sehr raschen Volumenzunahme in der Kammer durch die Verdampfung. Der daraus resultierende Druckanstieg überlagert offenbar den Druckabfall der Verdunstungskühlung. Ab einem Kammerdruck von $p_{0}=877$ mbar sind die Voraussetzungen für Flash Boiling nicht mehr gegeben. Hin-
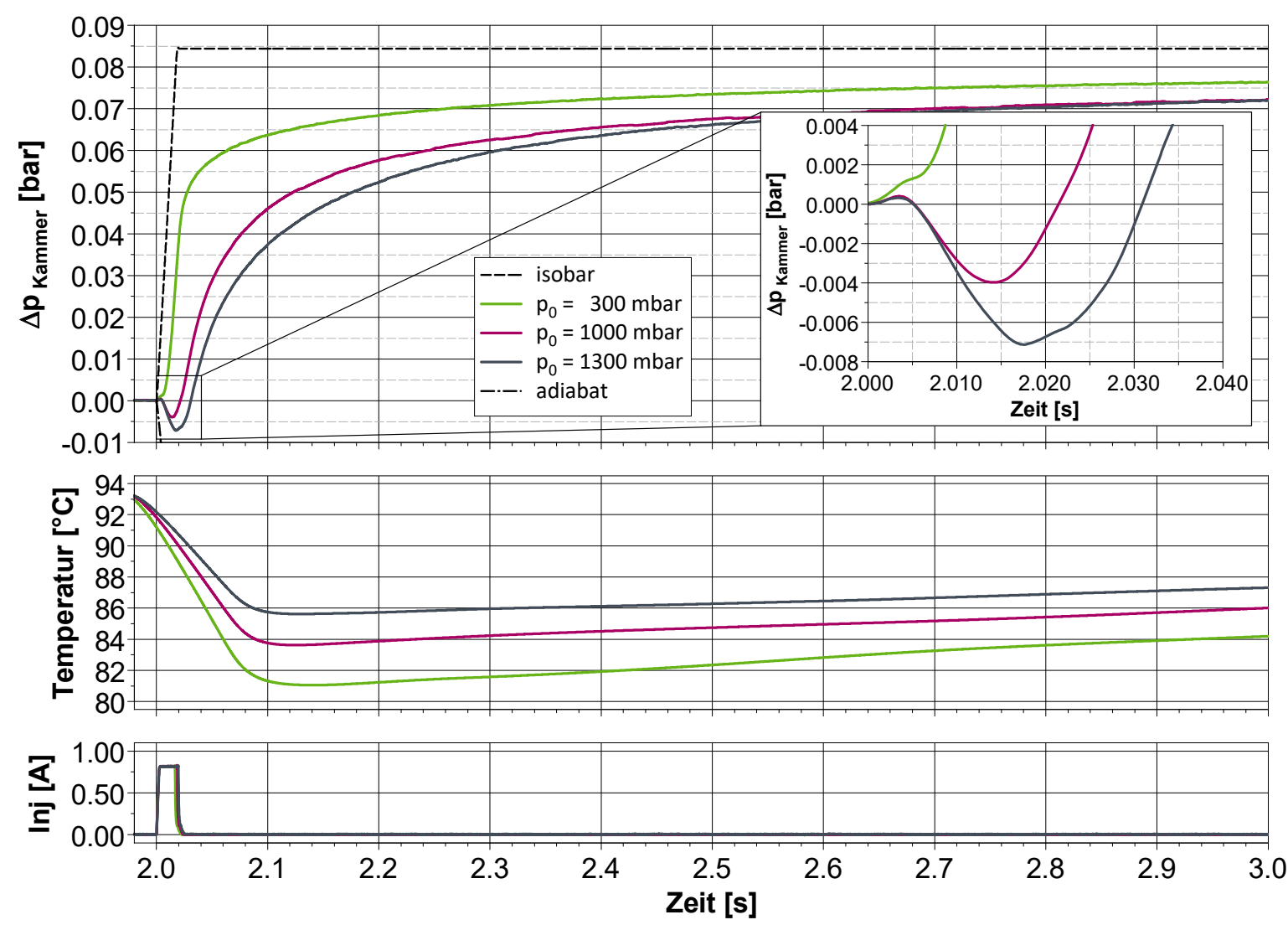

Abbildung 4.1.: Verdunstungsmessungen mit verschiedenen Kammerdrücken $\left(p_{0}\right)$ bei $T_{0}=93^{\circ} \mathrm{C}$ und konstanter Einspritzmasse $m_{i n j}=36 \mathrm{mg}$

sichtlich der Temperaturmessung muss konstatiert werden, dass die Temperaturänderungen nicht schnell genug erfasst werden können. Es gelingt nicht in der Zeitskala der Tropfenverdunstung, also innerhalb von $30 \mathrm{~ms}$, die Gastemperatur zu erfassen. Dies ist auf die im Vergleich zur umgebenden Kammerluft, trotz der angeschliffenen Messspitze, immer noch große Wärmekapazität des Thermoelements zurückzuführen. Dennoch kann die Temperaturmessung langsamere Trends wiedergeben und zur Plausibilisierung der Druckverläufe dienen. So ist beispielsweise erkennbar, dass mit geringerem Kammerdruck die Temperatur stärker abfällt. Dies ist insofern plausibel, als dass sich

${ }^{2}$ Flash Boiling nach Weber [119] bei $R_{p}=\frac{p_{\text {sat }}\left(T_{T r}\right)}{p_{\text {Kammer }}}>1$, hier $\frac{877 \mathrm{mbar}}{300 \mathrm{mbar}}=2,92$ 
bei geringerem Ausgangsdruck entsprechend weniger Stickstoffmasse in der Kammer befindet. Bei näherungsweise gleicher Wärmekapazität $c_{v}$ und gleicher notwendigen Verdunstungsenthalpie führt dies zu einer größeren Temperaturabsenkung.

\section{Vergleich verschiedener Injektorsprays}

Die Bewertung verschiedener Injektorsprays hinsichtlich ihrer Verdunstungsneigung erfolgt bei einem initialen Kammerdruck von $p_{0}=1000$ mbar. Zur Simulation eines betriebswarmen Motors wird die Verdunstungskammer auf $T_{0}=93^{\circ} \mathrm{C}$ konditioniert. In einer weiteren Messkampagne soll eine Kammertemperatur von $T_{0}=35^{\circ} \mathrm{C}$ die Bedingungen eines im Warmlauf befindlichen Motors nachbilden. Die zeitlich hoch aufgelösten Kammerdruckverläufe mit verschiedenen Injektoren sind für beide Starttemperaturen in Abbildung 4.2 geplottet. An dieser Stelle sei erwähnt, dass jede Kurve einen
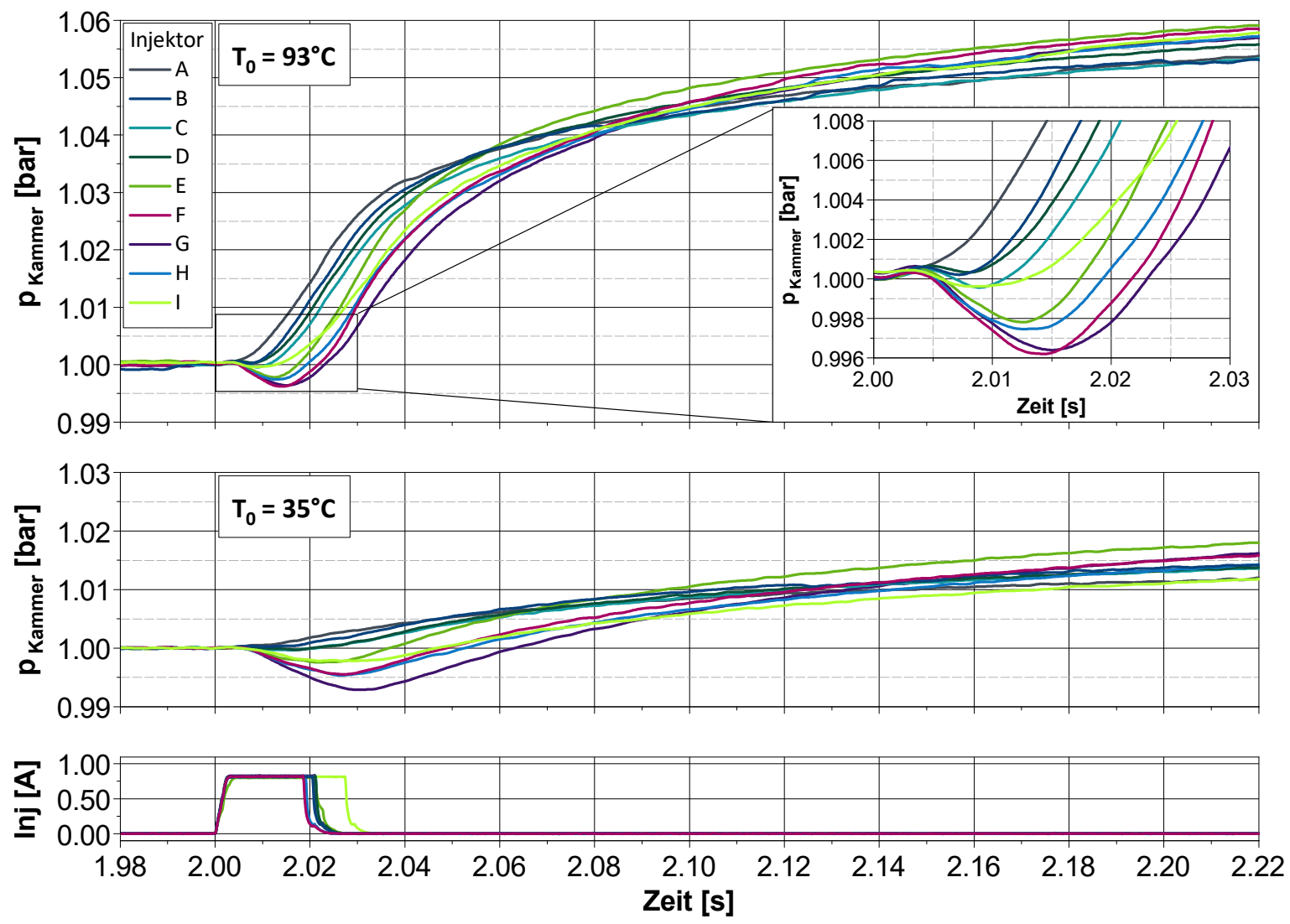

Abbildung 4.2.: Verdunstungsmessungen mit verschiedenen Injektor-Sprays bei $T_{0}=93^{\circ} \mathrm{C}$ (oben) und $T_{0}=35^{\circ} \mathrm{C}$ (mitte) bei konstanter Einspritzmasse $m_{i n j}=36 \mathrm{mg}$ bei $1000 \mathrm{mbar}$ Kammerdruck

Mittelwert aus drei Messungen darstellt. Um sicherzustellen, dass mit jedem Injektor dieselbe Einspritzmasse von $m_{i n j}=36 \mathrm{mg}$ abgesetzt wird, erfolgt eine entsprechende 
Anpassung der elektrischen Ansteuerdauer anhand vorab ermittelter Injektorkennlinien ( „, $q_{d y n}$-Kennlinie“). Die Zeitspanne für Tropfenverdunstung, gekennzeichnet durch den Druckabfall unmittelbar nach Beginn der Einspritzung, ist in Relation zur nachfolgenden Wandfilmverdunstung sehr kurz. Zum einen ist je nach Spraytargeting nach bereits $10 \mathrm{~ms}$ mit dem ersten Wandkontakt der Tropfen zu rechnen, wie aus Penetrationsmessungen und High-Speed Videos im Einlasskanal hervorgeht, zum anderen erfordert die Wandfilmverdunstung auffällig viel Zeit, sodass der Enddruck von $p_{\infty}=1091$ mbar im dargestellten Zeitbereich von $220 \mathrm{~ms}$ noch gar nicht erreicht wird. Letzteres ist unter anderem auf inhomogene Wandfilmdicken, z.B. durch Akkumulation von Kraftstoff im Ventilspalt, zurückzuführen. Dort steht im Vergleich zu dünnen, großen Wandfilmen, wenig Oberfläche zur Verdunstung zur Verfügung.

Die deutlich verschiedenen Druckverläufe der untersuchten Injektoren, insbesondere während der ersten $50 \mathrm{~ms}$, zeigen, dass es mittels der Verdunstungskammer gelingt, Rückschlüsse auf die Verdunstungsneigung verschiedener Sprays anzustellen. Je nach Sprayauslegung ist die Tropfenverdunstung stark ausgeprägt, oder so gering, dass überhaupt kein Druckabfall messbar ist. Betrachtet man die Gradienten der Druckverläufe im weiteren Verlauf, also die Annäherung an den Enddruck, sind auch hier Unterschiede zwischen den Injektorsprays erkennbar. Bei den Messungen mit $T_{0}=35^{\circ} \mathrm{C}$ sind deutlich flachere Druckverläufe zu verzeichnen. Dies lässt auf eine erheblich langsamere Verdunstung schließen und stellt einen Ausblick auf die großen Herausforderungen der Gemischbildung mittels SRE bei kaltem Motor dar. Im Folgenden wird durch Berechnung der in Kapitel 3.1.3 vorgestellten Bewertungszahl $K_{D V A}$ eine quantitative Bewertung der Verdunstungsneigung der verschiedenen Sprays angestellt.

Eine große Kennzahl aus der Druckverlaufsanalyse deutet auf eine gute Verdunstungsneigung eines Sprays hin und spricht somit für eine gelungene Spray-Auslegung für die vorliegende Einlasskanalgeometrie. Bei direktem Vergleich der ermittelten Kennzahlen in Abbildung 4.3 von warmer und kalter Kammer ist die bessere Verdunstungsneigung bei höherer Temperatur leicht ersichtlich. Bezüglich der Sprayeigenschaften fällt auf, dass Injektoren mit großem $\beta$-Winkel und somit auch kleinerem SauterTropfendurchmesser SMD vorteilhaft abschneiden. Die Injektoren E, F, G und H erscheinen bei beiden Kammertemperaturen vielversprechend. Insbesondere Injektor G, welcher auch bei kaltem Motor eine vergleichsweise hohe $K_{D V A}$ erreicht, wird daher bei allen weiteren Untersuchungen am Vollmotor und Transparentmotor berücksichtigt.

Es sei aber auch hervorgehoben, dass kleine Tropfendurchmesser, gekennzeichnet durch den SMD, eine hinreichende aber keine ausreichende Bedingung für eine gute Verdunstungsneigung darstellen. So erreicht Injektor I mit dem kleinsten aller Sauterdurchmesser nur durchschnittliche Bewertungszahlen. Das unpassende Spray-Targeting mit einer einzelnen Spraykeule mit zentraler Ausrichtung auf den Trennsteg ist dafür verantwortlich. Dies unterstreicht die Bedeutung und Notwendigkeit einer optimierten Sprayauslegung („Spray-Targeting“) im Kontext der Gemischbildung. 

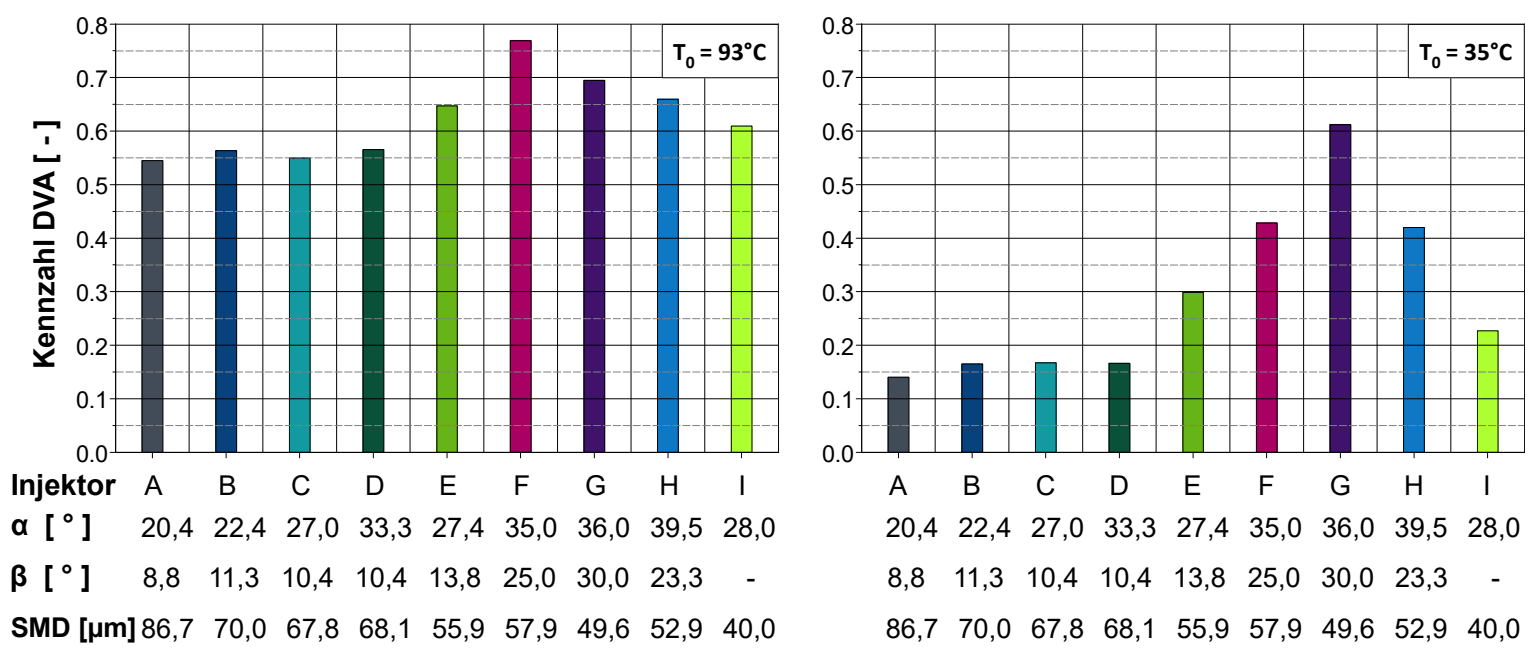

Abbildung 4.3.: Kennzahl zur Bewertung der Verdunstungsneigung verschiedener Sprays bei warmer Kammer (links) und kalter Kammer (rechts)

\subsubsection{Analyse der Strömungsverhältnisse am Einlassventil mittels Ladungswechselrechnung}

Bei Ottomotoren mit Saugrohreinspritzung stellt der Ladungswechsel einen wesentlichen Teilprozess der Gemischbildung dar. Zur Analyse der Strömungsverhältnisse an den Einlassventilen während des Ladungswechsels werden Ladungswechselrechnungen auf Basis von Druckverlaufsanalysen mit dem Software-Tool BeCat (Bosch Engine Combustion Analysis Tool) angestellt. Um Ladungswechselrechnungen durchführen zu können, ist es erforderlich sowohl den Druck im Einlasskanal als auch den Druck im Auslasskrümmer zylinderindividuell und zeitlich hoch aufgelöst zu erfassen. Außerdem müssen neben den Ventilhubkurven die effektiven Strömungsquerschnitte $A_{\text {eff }}=\alpha_{k} A_{k}$ für Einlass- und Auslassventile in Abhängigkeit des Ventilhubes bekannt sein. Unter der Annahme dass eine reibungsfreie, isentrope Strömung über die Ventile vorliegt, kann beispielsweise der Massenstrom vom Einlasskanal (mit $p_{1}=p_{\text {ein }}$ ) in den Brennraum (mit $p_{2}=p_{Z y l}$ ) bestimmt werden.

$$
\frac{d m}{d t}=A_{e f f} \frac{p_{1}}{\sqrt{R T_{1}}} \sqrt{\frac{2 \kappa}{\kappa-1}\left[\left(\frac{p_{2}}{p_{1}}\right)^{\frac{2}{\kappa}}-\left(\frac{p_{2}}{p_{1}}\right)^{\frac{\kappa+1}{\kappa}}\right]}
$$

In Abbildung 4.4 sind die Messdaten der Druckverläufe sowie die berechneten Massenströme über die Ventile für einen Teillastbetriebspunkt dargestellt. Zum besseren Verständnis sind auch die Ventilerhebungskurven, sowie die Zeitspanne einer vorgelagerten Einspritzung, eingezeichnet. Bei diesem Teillastbetriebspunkt mit einem indizierten Mitteldruck von IMEP $=2,5$ bar herrscht ein mittlerer Saugrohrdruck von $p_{S R}=600$ mbar. Da zum Zeitpunkt des Öffnens der Einlassventile („EV̈̈“) der Druck im Brennraum $p_{Z y l}$ noch auf dem Niveau des Abgasgegendrucks von etwa 

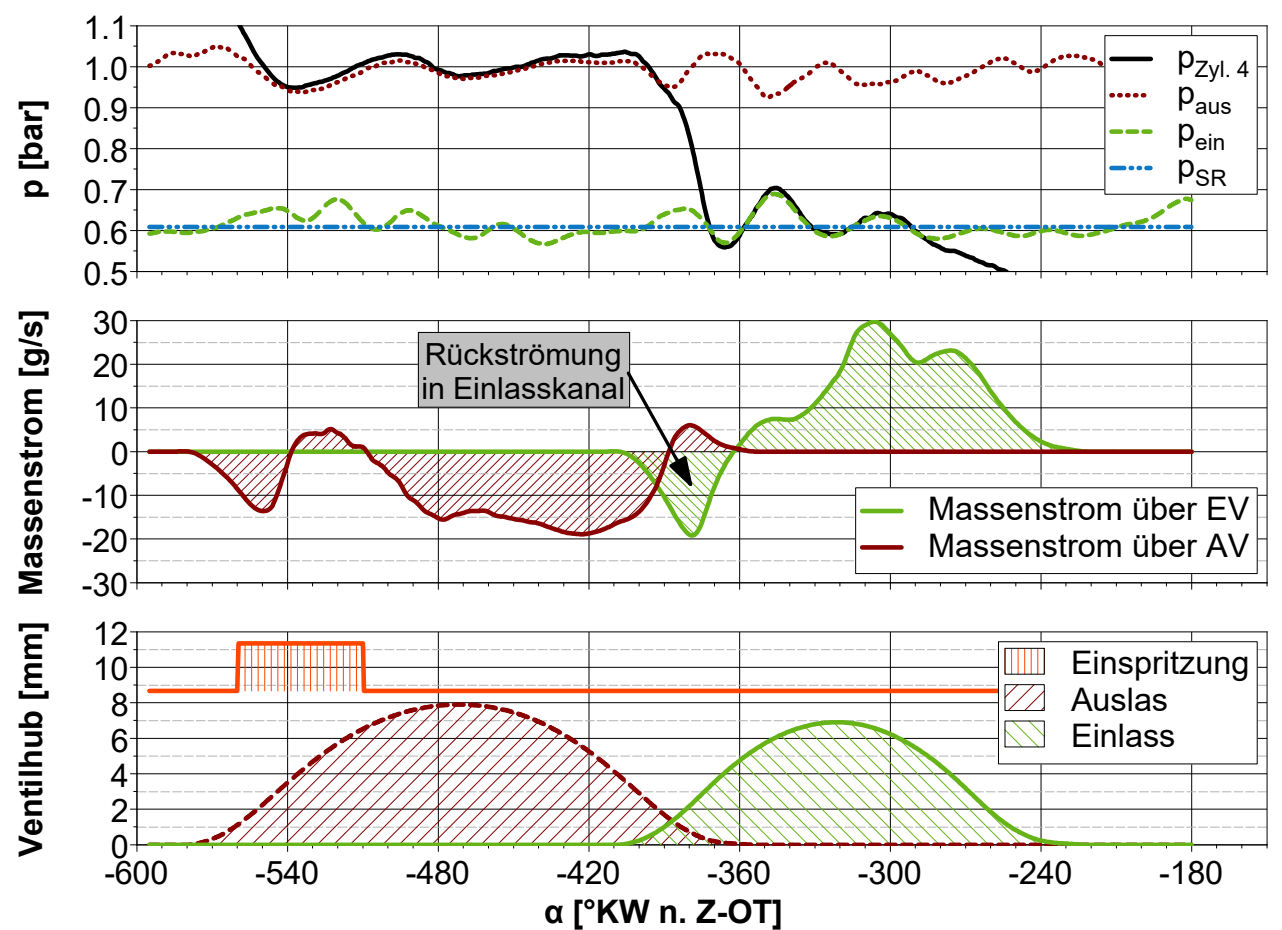

Abbildung 4.4.: Ladungswechsel-Analyse bei $N=2000 \mathrm{~min}^{-1}, I M E P=2,5 \mathrm{bar}$ und $p_{S R}=600$ mbar am Vollmotor

$p_{\text {aus }}=1000$ mbar liegt, kommt es so lange zu einer Rückströmung aus dem Brennraum in den Einlasskanal bis der Druckausgleich zwischen Brennraum und Einlasskanal bzw. Saugrohr abgeschlossen ist. Bei Betrachtung der grün schraffierten Fläche unterhalb der Massenstromkurve für das Einlassventil wird deutlich, dass die vorliegende Rückströmung in Relation zum Gesamtmassenstrom über das Einlassventil einen wesentlichen Anteil darstellt. Diese Rückströmung aus heißem Abgas leistet einen wertvollen Beitrag für die Verdunstung der flüssigen Kraftstoffwandfilme im Einlasskanal und an den Einlassventilen.

Im Folgenden soll derselbe Sachverhalt für einen Betriebspunkt mit höherer Motorlast untersucht werden. Die gemessenen Druckverläufe und die berechneten Massenströme über die Ventile für einen indizierten Mitteldruck von IMEP $=12$ bar und einem mittleren Saugrohrdruck von $p_{S R}=1100$ mbar, also einen Betriebspunkt mit leichter Aufladung, sind in Abbildung 4.5 aufgetragen. Da nun der Druck im Einlasskanal $p_{\text {ein }}$ zum Zeitpunkt „EVö“ nahezu auf demselben Druckniveau wie der Brennraumdruck $p_{Z y l}$ liegt, entwickelt sich nur noch eine kurze und schwach ausgeprägte Rückströmung. In Relation zum anschließenden Massenstrom vom Einlasskanal in den Brennraum (Vorwärtsströmung), ist diese Rückströmung sehr klein. Folglich ist kein nennenswerter Beitrag zur Gemischbildung mehr zu erwarten. Inwiefern eine Rückströmung in den Einlasskanal die Gemischbildung begünstigt, wird im nächsten Abschnitt anhand von Aufnahmen mittels High-Speed Video-Endoskopie (HSVE) im Saugrohr analysiert. 

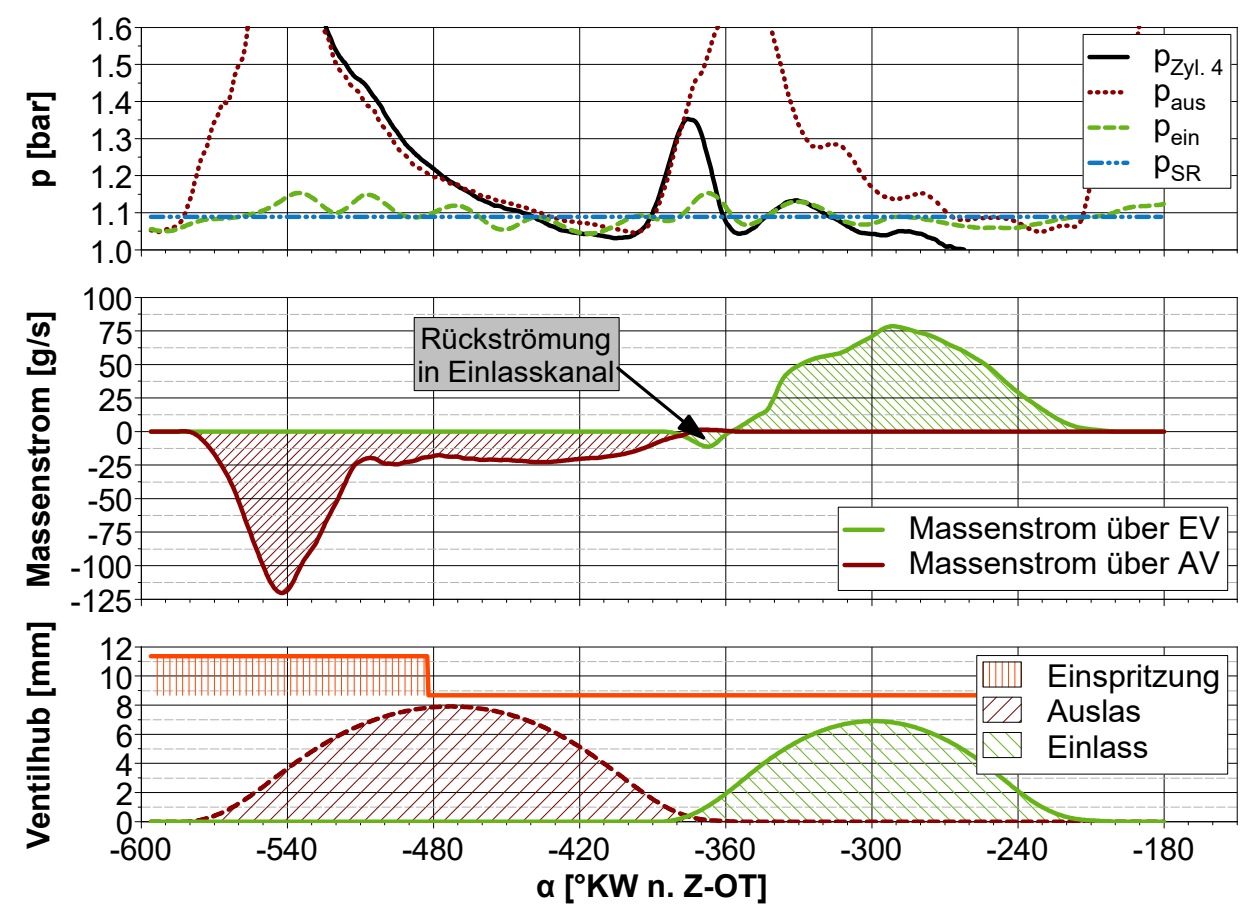

Abbildung 4.5.: Ladungswechsel-Analyse bei $N=2000 \mathrm{~min}^{-1}, \quad I M E P=12 \mathrm{bar}$ und $p_{S R}=1100$ mbar am Vollmotor

Anhand einer Vollmotormessung an einem Betriebspunkt mit Teilast bei IMEP $=7.6$ bar und $p_{S R}=889$ mbar kann die Auswirkung der beschriebenen Rückströmung auf die Gemischbildung anschaulich studiert werden, wie Abbildung 4.6 zeigt. Zu sehen sind Endoskopieaufnahmen der High-Speed Kamera 2 im Einlasskanal eines beispielhaften Ladungswechsels. Mittels der Trajektorie eines einzelnen Tropfens kann die Rückströmung veranschaulicht werden. Der mit einem weißen Kreis markierte, nahezu ruhende, einzelne Tropfen entstand gegen Ende der Einspritzung und entstammt nicht dem Hauptspray.

a) Die ersten Tropfen des Kraftstoffsprays erreichen die Einlassventile.

b) Kraftstoff sammelt sich an den geschlossenen Einlassventilen. Die Einspritzung ist beendet.

c) Die Einlassventile öffnen sich („EVö“). Die Rückströmung entwickelt sich.

d) Die Strömungsgeschwindigkeit der Rückströmung steigt weiter an.

e) Die Mehrzahl aller flüssigen Tropfen wurde durch die Rückströmung von den Einlassventilen zurück in den Einlasskanal gedrängt.

f) Übergang von Rückströmung zu Vorwärtsströmung.

g) Die Strömungsgeschwindigkeit der Vorwärtsströmung nimmt zu. Die meisten Kraftstofftropfen sind verdunstet.

h) Wenige, übrig gebliebene, flüssige Kraftstofftropfen passieren die Einlassventile.

Es wird also deutlich, dass an den Einlassventilen befindliche Wandfilme in den Einlasskanal zurückgedrängt werden können. Somit sinkt das Risiko, dass jene Wandfilme in 


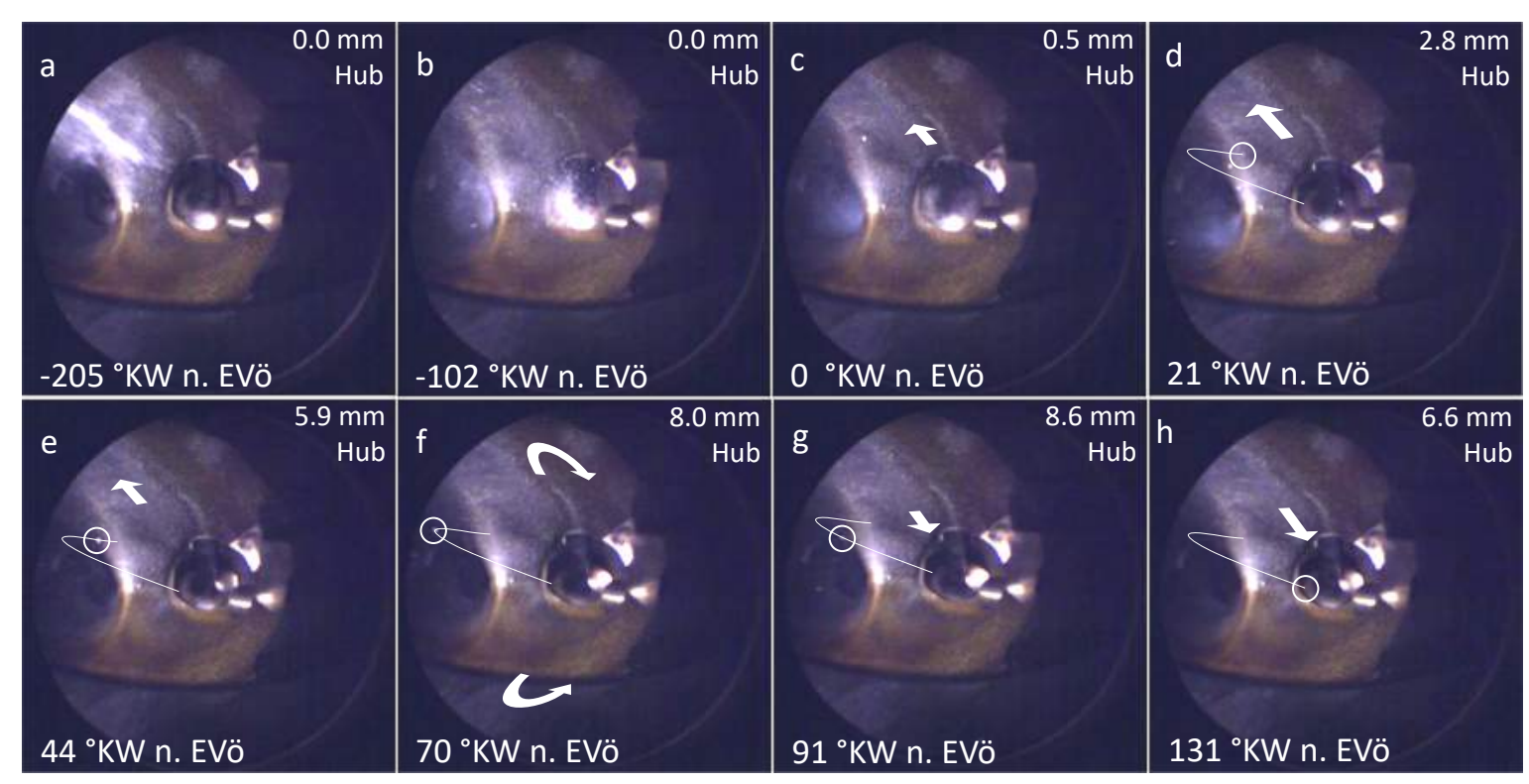

Abbildung 4.6.: High-Speed Visualisierung der Rückströmung am Vollmotor bei $\mathrm{N}=1750 \mathrm{~min}^{-1}, p_{S R}=889$ mbar und cVI mit Injektor A

flüssiger Form in den Brennraum gelangen und potentielle Quellen für PN-Emissionen darstellen. Außerdem sorgt das rückströmende, heiße Abgas für günstigere Verdunstungsbedinungen im Einlasskanal, da hierdurch die Gastemperatur steigt. Um den großen Einfluss der Temperatur auf die Verdunstungszeit von Kraftstoffen zu unterstreichen, sei an dieser Stelle nochmals auf die Ergebnisse in Kapitel 4.1.1, sowie auf die Literatur verwiesen, welche in Kapitel 2.2.4 diskutiert wird.

Inwiefern sich ein steigender Saugrohrdruck $p_{S R}$, also eine steigende Motorlast und somit eine kleiner werdende Rückströmung auf die Wandfilme an den Einlassventilen und letztlich auf die Partikelemission auswirkt, soll im folgenden Kapitel 4.2.1 erörtert werden. 


\title{
4.2. Analyse und Optimierung der Gemischbildung im Brennraum
}

\author{
Innermotorische Einflussgrößen auf PN
}

\subsubsection{Motorlast - gekennzeichnet durch den Saugrohrdruck}

Zur Analyse des Einflussparameters Motorlast wird bei stationären Messungen der Saugrohrdruck $p_{S R}$ bei verschiedenen Motordrehzahlen und konventionellem, vorgelagertem Einspritztiming sukzessive erhöht und die Partikelkonzentration im Abgas erfasst. Sowohl mit Injektor A, als auch mit Injektor B wird im linken Diagramm von Abbildung 4.7 deutlich, wie steil sich die Abhängigkeit von PN gegenüber der Motorlast darstellt. Im Vergleich zu BDE-Motoren besteht eine viel sensiblere Abhängigkeit
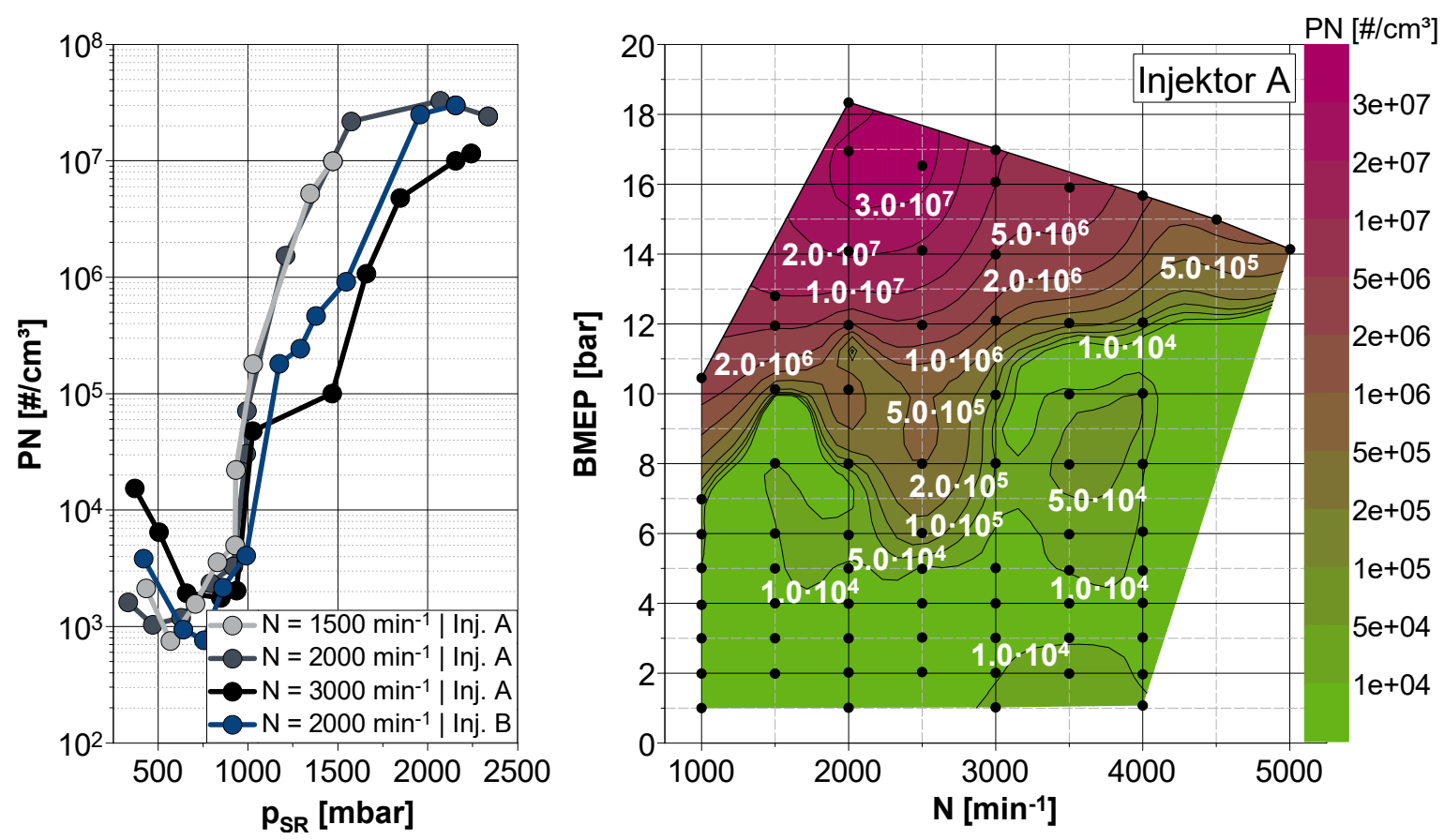

Abbildung 4.7.: PN-Emissionen bei steigender Motorlast mit vorgelagerter Einspritzung (cVI), dargestellt als Lastschnitte bei konstanten Drehzahlen mit Injektor A und B (links) und als Kennfeld mit Injektor A (rechts)

der PN-Emissionen vom Saugrohrdruck und somit von der Motorlast. Hervorzuheben ist ein besonders starker Anstieg der PN-Emissionen oberhalb von $p_{S R} \geq 1000 \mathrm{mbar}$, bzw. einem effektiven Mitteldruck von BMEP $\geq 9$ bar in der Darstellung der Messdaten mit Injektor A als Kennfeld (rechts). Dieser Schwellwert kann durch die in Kapitel 4.1.2 diskutierte Rückströmung erklärt werden, da bei Saugrohrdrücken oberhalb des Abgasgegendrucks an den Einlassventilen kein negatives Spülgefälle mehr existiert und 
die Rückströmung verschwindet. Bemerkenswert ist außerdem, dass sich das Spektrum der PN-Emissionen über fünf Größenordnungen $\left(10^{2} \ldots 10^{7}\right)$ erstreckt.

Bei geringer Motorlast sprechen PN-Konzentrationen von weniger als $10^{4} \# / \mathrm{cm}^{3}$ für eine ausgesprochen gute Gemischbildung. Trotz der Vorlagerung des Kraftstoffes an den Einlassventilen gelangen offenbar keine Wandfilme in den Brennraum. Die Auswirkungen eines hohen Saugrohrdrucks auf die Gemischbildung im Brennraum werden durch HSVE-Aufnahmen deutlich, die in Abbildung 4.8 visualisiert sind. In der linken
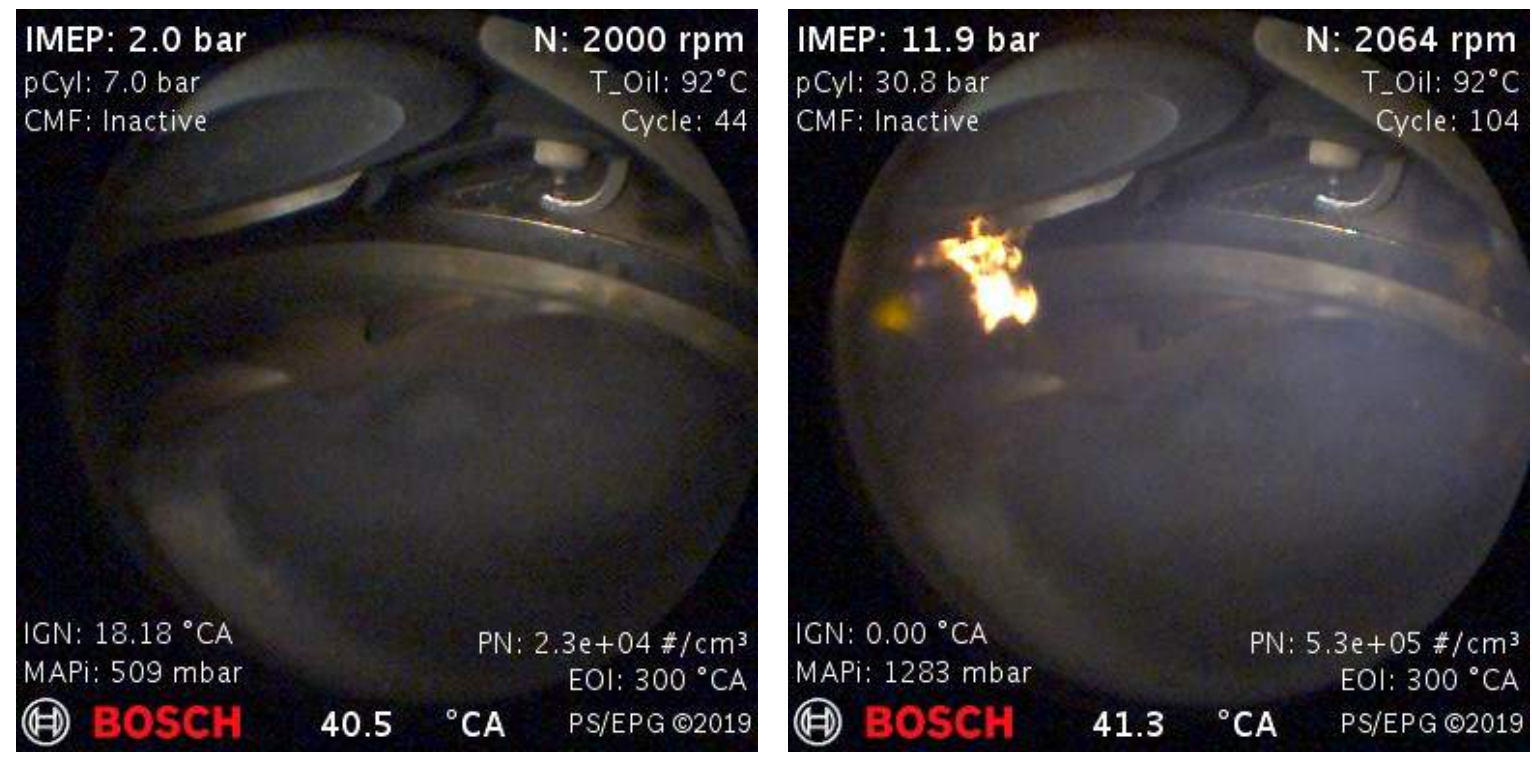

Abbildung 4.8.: Gegenüberstellung von Verbrennungen mittels HSVE aus einer Messung mit niedriger Motorlast bei $N=2000 \mathrm{~min}^{-1}$, IMEP $=2 \mathrm{bar}$ und $p_{S R}=509$ mbar (links) und hoher Motorlast bei $N=2064 \mathrm{~min}^{-1}$, $\mathrm{IMEP}=12$ bar und $p_{S R}=1283$ mbar (rechts) mit Injektor B

Bildhälfte von Abbildung 4.8 ist bei niedriger Motorlast von IMEP $=2$ bar eine gut homogenisierte, vorgemischte Verbrennung dargestellt. Da letztere hauptsächlich ultraviolettes (UV) Licht emittiert, kann die im sichtbaren Wellenlängenbereich arbeitende High-Speed Kamera nur den Brennraumhintergrund erfassen. Bei hoher Motorlast von IMEP $=12$ bar sind in der rechten Bildhälfte hingegen gelbliche Diffusionsflammen an der Unterseite des Einlassventils erkennbar. Durch den hohen Saugrohrdruck von $p_{S R}=1283$ mbar kann sich beim Ladungswechsel keine Rückströmung mehr entwickeln, sodass Kraftstoffwandfilme, entstanden durch die vorgelagerte Einspritzung, durch die Ansaugströmung vom Einlasskanal in den Brennraum transportiert werden und dort offenbar zu lokal fetten Zonen führen. Es kommt folglich zu diffusionsgesteuerten Verbrennungen, welche mit Rußemissionen einhergehen.

Für eine Analyse des Einflussparameters Saugrohrdruck $\left(p_{S R}\right)$ auf die Bildung von Kraftstoffwandfilmen im Brennraum, und im Speziellen an der Unterseite der Einlassventile, ist die gute optische Zugänglichkeit des Brennraumdaches am Transparent- 
motor mit der Perspektive „1-V" von Vorteil. Zur Erhaltung eines stöchiometrischen Gemisches mit $\lambda=1.0$ wird für Messungen mit steigendem Saugrohrdruck auch die eingespritzte Kraftstoffmasse entsprechend erhöht. Die durch Kraftstoffwandfilme benetzten Flächenanteile der Einlassventile von Zylinder vier sind auf der linken Seite von Abbildung 4.9 für 14 Arbeitsspiele dargestellt. Als Bezugsfläche dient die Gesamtfläche

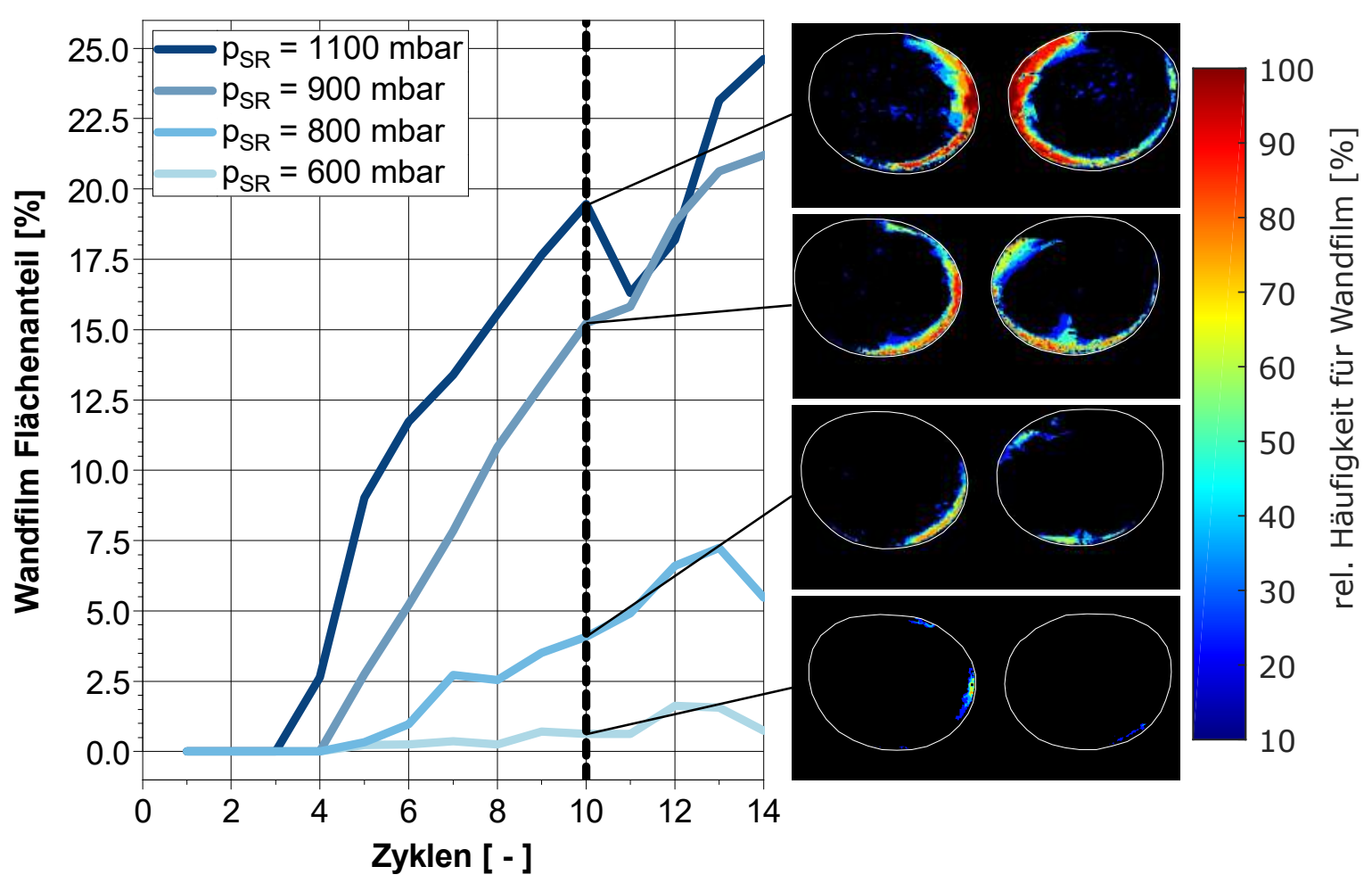

Abbildung 4.9.: Fläche der Kraftstoffwandfilme an der Unterseite der Einlassventile (Perspektive "1-V“) am Transparentmotor bei $\mathrm{N}=2000 \mathrm{~min}^{-1}$ und steigendem $p_{S R}$ mit Injektor B

der beiden Einlassventilunterseiten, welche rechts durch weiße Linien gekennzeichnet sind. Auf der rechten Bildhälfte sind statistische Auswertungen der Benetzungswahrscheinlichkeit innerhalb der ersten zehn Arbeitsspiele abgebildet. Es werden jeweils vier Saugrohrdrücke betrachtet.

Ein steigender Saugrohrdruck $p_{S R}$ führt demnach zu einer erheblichen Zunahme der brennraumseitigen Einlassventilbenetzung. Während die Wandfilme bei $p_{S R}=600 \mathrm{mbar}$ nahe an der Detektionsgrenze des Analyseverfahrens liegen, werden bei $p_{S R}=1100 \mathrm{mbar}$ nach zehn Zyklen nahzu $20 \%$ der Ventilfächen benetzt. Die Wirksamkeit der in Kapitel 4.1 .2 beschriebenen Rückströmung bei geringen Saugrohrdrücken zur Reduktion von Wandfilmen im Brennraum wird hier ersichtlich. Zum einen erfolgt ein Zurückdrängen des flüssigen Kraftstoffs in den Einlasskanal, zum anderen sorgt das heiße, rückströmende Restgas für günstigere Verdunstungsbedingungen. Letzteres kommt allerdings am ungefeuerten Transparentmotor nicht zum Tragen. Der positive Effekt 
erhöhter Temperatur auf Verdunstungsprozesse ist jedoch am Beispiel der Verdunstungskammer in Kapitel 4.1.1 zu sehen. Verschwindet die Rückströmung mit steigendem Saugrohrdruck, kommt es am inneren und unteren Rand der Einlassventile zu verstärkter Wandfilmbildung.

Die dargestellten Wandfilmflächen an den Einlassventilen des Transparentmotors werden für Fragestellungen am betriebswarmen, gefeuerten Vollmotor vermutlich überschätzt, da die Einlassventile am Transparentmotor deutlich kälter sein dürften als am gefeuerten Vollmotor. Vergleichende Rückschlüsse zum prinzipiellen Einfluss von bestimmten Parametern auf Wandfilmbildung, wie dem Saugrohrdruck, sind aber dennoch möglich. So wird deutlich, dass ein hoher Saugrohrdruck zu größeren Wandfilmen an den Einlassventilen führt und damit das Risiko für die Partikelentstehung an dieser Stelle erheblich steigt.

\subsubsection{Steuerzeit Einlassnockenwelle}

Sowohl die Untersuchungen in Kapitel 4.1.2 zu den Strömungsverhältnissen am Einlassventil als auch die Versuche zur Variation des Saugrohrdrucks in Kapitel 4.2.1 zeigen die Wirksamkeit einer Rückströmung in den Einlasskanal im Hinblick auf die Gemischbildung. Diese Rückströmung ist mit steigender Motorlast immer geringer ausgeprägt und verschwindet oberhalb der saugmotorischen Volllast nahezu vollständig. Daher stellt sich die Frage, ob es möglich ist, den Kennfeldbereich zu höheren Lasten hin auszuweiten, in welchem diese Rückströmung die Gemischbildung unterstützt. Im diesem Teilkapitel soll erörtert werden, ob die Variation des Öffnungszeitpunktes der Einlassventile einen Ansatz hierfür darstellen kann.

In Abbildung 4.10 ist die Auswirkung einer Früh-Verstellung des Öffnungszeitpunktes der Einlassventile (EVö) auf die Rückströmung in den Einlasskanal dargestellt. Das Öffnen (und Schließen) der Auslassventile wird hierbei zum frühestmöglichen Zeitpunkt festgehalten $\left(\mathrm{AVs}=-23^{\circ} \mathrm{KW}\right)$, um ein möglichst hohes Druckniveau im Brennraum anzustreben. Für mehrere Messungen mit verschiedenen Öffnungszeitpunkten der Einlassventile wird anhand von Ladungswechselrechnungen der Massenstrom über die Ein- und Auslassventile berechnet. So wird deutlich, dass durch frühes Öffnen der Einlassventile auch bei einem Ladedruck von $p_{S R}>1200$ mbar eine Rückströmung in den Einlasskanal erzeugt werden kann.

Die erwartete positive Auswirkung in Form von geringeren Partikelemissionen stellt sich jedoch nicht ein. Ein Grund hierfür stellt der bei frühem EVö und größerer Ventilüberschneidung geringere Liefergrad dar. Dies führt zu einem höheren Saugrohrdruck, welcher zur Erreichung derselben Motorlast von IMEP $=12,8$ bar erforderlich ist. Ein höherer Saugrohrdruck wirkt sich wiederum negativ auf die Verdunstungsbedingungen in Einlasskanal und Brennraum nach dem Schließen der Einlassventile (EVs) aus. Beim Vergleich der sich ähnelnden Verläufe von Saugrohrdruck $p_{S R}$ (oben) und Partikelkonzentration $P N$ (Mitte) wird die schädliche Auswirkung hoher Saugrohrdrücke 

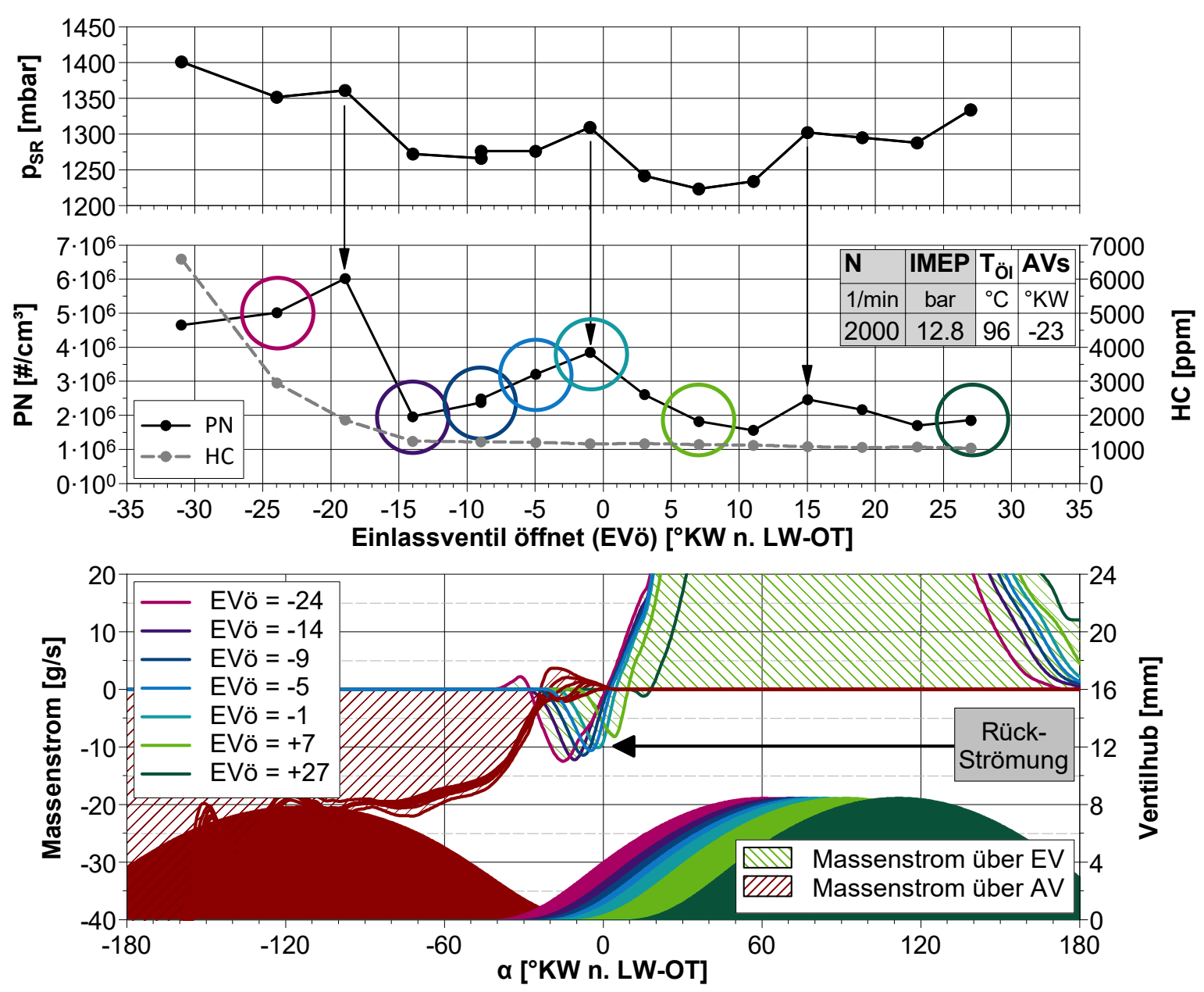

Abbildung 4.10.: Variation der Steuerzeit Einlassventil öffnet (EVö) im Hinblick auf die Rückströmung in den Einlasskanal sowie auf PN mit cVI

auf die Gemischbildung offensichtlich. Weiterhin gilt es zu bedenken, dass die Masse an rückwärts strömendem Abgas in Relation zur vorwärts strömenden Gemischmasse auch bei sehr frühem EVö klein ausfällt.

Eine weitere Einschränkung stellen die mit zunehmender Ventilüberschneidung rasch ansteigenden HC-Emissionen dar. Gerade bei vorgelagerter Einspritzung (cVI) besteht hierbei die Gefahr, dass Kraftstoff direkt die Auslassventile passiert und nicht an der Verbrennung teilnimmt. Daher sind Steuerzeiten mit zu großer Ventilüberschneidung im Kennfeldbereich mit Ladedruck $p_{S R}>p_{A b g}$. aufgrund des positiven Spülgefälles unbedingt $\mathrm{zu}$ vermeiden.

Die Möglichkeiten bei Motorlasten oberhalb der saugmotorischen Volllast mit Hilfe der Ventilsteuerzeiten die Gemischbildung durch eine erzwungene Rückströmung zu verbessern und dadurch die Partikelemission zu reduzieren, sind mit dem verwendeten Forschungsmotor aus diesem Grunde sehr eingeschränkt. Für künftige, weiterführende Untersuchungen wären jedoch spezielle Ventilerhebungskurven denkbar, welche eine 
Rückströmung gezielt ermöglichen. Für weitere Informationen zu diesem Gedankengang sei auf die veröffentlichte Erfindungsmeldung von Samenfink und Schück im Anhang A.2 verwiesen.

\subsubsection{Einspritztiming}

Im folgenden Abschnitt wird der Einfluss des Einspritztimings auf die Partikelemissionen bei betriebswarmem Motor mit Injektor B erörtert. Hierfür werden mehrere Motorbetriebspunkte herangezogen, welche in einer Abschlussarbeit von Wang [118] anhand von Fahrzeugmessungen in drei verschiedenen Fahrzyklen ermittelt wurden. Jeder Datenpunkt in Abbildung 4.11 steht für ein Ende der elektrischen Ansteuerung (EOI) bezogen auf den oberen Totpunkt im Ladungswechsel (LW-OT). Die teils

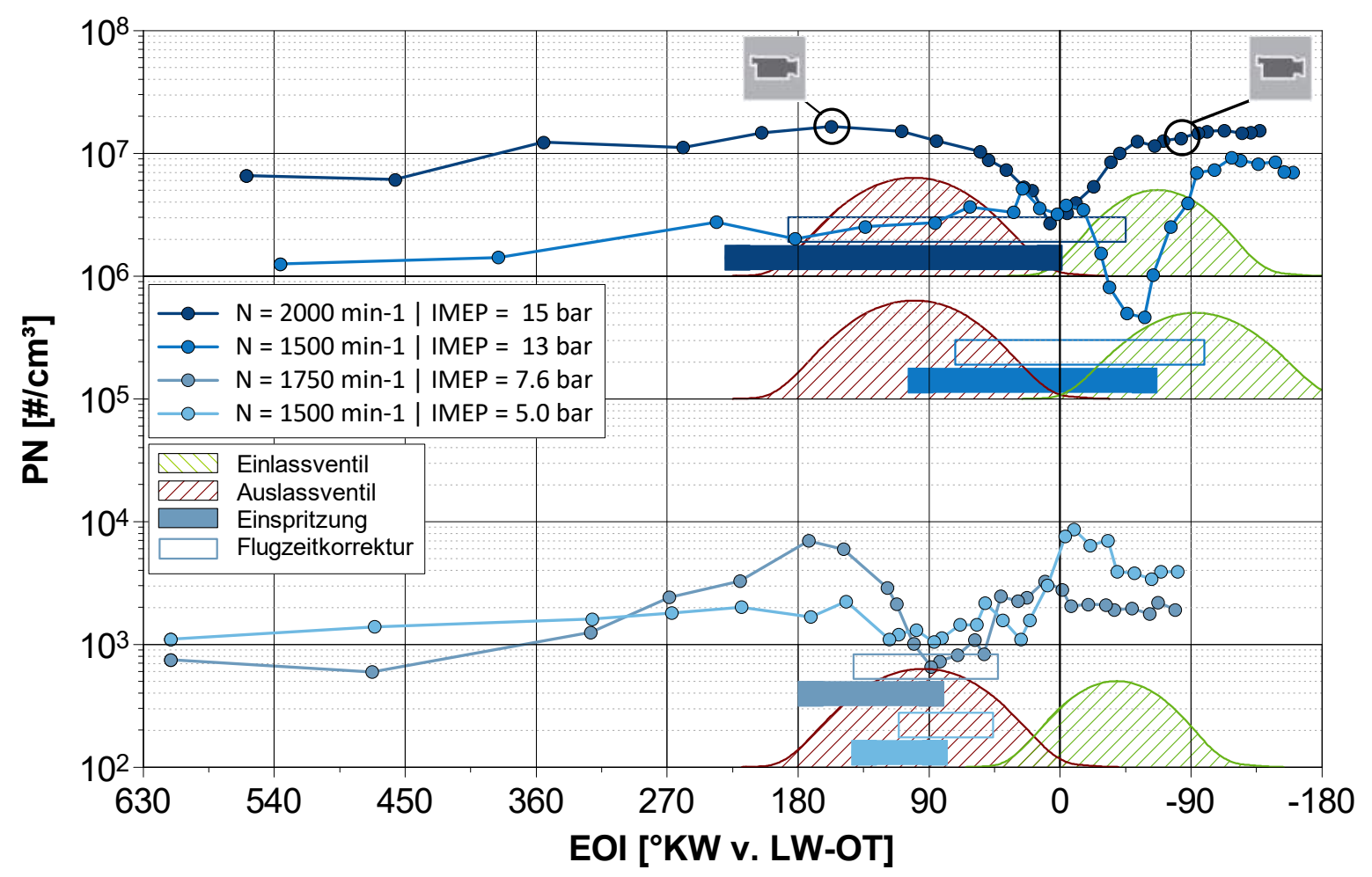

Abbildung 4.11.: Variationen des Einspritzende (EOI) bezüglich LW-OT für diverse Betriebspunkte mit Injektor B bei warmem Motor $\left(T_{\ddot{\mathrm{O}} l}=93^{\circ} \mathrm{C}\right)$

verschiedenen Steuerzeiten der jeweiligen Betriebspunkte sind anhand der Ventilerhebungskurven gekennzeichnet. Für jeden der vier Betriebspunkte ist eine beispielhafte Einspritzdauer sowie die Flugzeitkorrektur am jeweils PN-optimalen EOI dargestellt.

Zunächst ist festzustellen, dass für beide Betriebspunkte mit geringer Motorlast, also IMEP $=5,0$ bar und IMEP $=7,6$ bar sehr geringe Partikelemissionen von $P N<$ $10^{4} \mathrm{H} / \mathrm{cm}^{3}$ ermittelt werden, unabhängig vom Einspritztiming. Folglich kann das EOI 
für diese Betriebspunkte hinsichtlich PN-Emissionen beliebig gewählt und das Augenmerk auf andere Parameter wie Kraftstoffverbrauch, Motorlaufruhe oder gasförmige Schadstoffemissionen wie $\mathrm{HC}, \mathrm{CO}$ und $\mathrm{NO}_{x}$-Emissionen gerichtet werden. Ein PNoptimales Einspritztiming liegt bei niedrigen Lasten im vorgelagerten Zeitfenster. Wie bereits erwähnt jedoch auf irrelevant niedrigem Niveau. Bei höherer Motorlast von IMEP $=13$ bar und IMEP $=15$ bar sind deutlich höhere Partikelkonzentrationen (Faktor 1000) zu verzeichnen. Hier liegen die PN-Optima etwas später und somit anteilig (zu etwa 20 - 50\%) im saugsynchronen Zeitbereich. Das heißt, dass einige Tropfen an den sich gerade öffnenden Einlassventilen vorbei in den Brennraum eingespritzt werden. Noch spätere Einspritztimings mit einem höheren saugsynchronen Anteil führen entweder zu nachteiligen oder gleichbleibenden PN-Emissionen, verglichen mit einer vorgelagerten Einspritzstrategie.

Die Ausnutzung der Gasdynamik zum Zeitpunkt des Einlassventil-Öffnens stellt eine hypothetische Erklärung für die Lage der Optima dar. Auch wenn die Rückströmung bei hoher Motorlast, wie in Kapitel 4.1.2 gezeigt, nur geringfügig ausgeprägt ist, ist ein positiver Effekt auf die Gemischbildung vorstellbar. Einige Spray-Tropfen werden hierbei möglicherweise von der kurzlebigen Rückströmung erfasst, noch vor einem ersten Wandkontakt. Neben dieser Hypothese stellt sich außerdem die Frage, ob der Entstehungsort für Partikel im Brennraum für vorgelagerte und saugsynchrone Einspritzung gleich oder verschieden ist. Diesen Fragen soll im Folgenden mit Hilfe der HSVE sowie mit Wandfilm-Analysen am Transparentmotor nachgegangen werden.

In Abbildung 4.12 werden zwei beispielhafte Verbrennungen einer vorgelagerten Einspritzung (links) und einer saugsynchronen Einspritzung (rechts) für den Motorbetriebspunkt mit IMEP $=15,6$ bar gegenübergestellt. Die beiden hier dargestellten Einspritztimings sind in Abbildung 4.11 mit einem grauen Kamera-Symbol markiert. Obwohl die PN-Emissionen bei diesen beiden Messungen auf vergleichbar hohem Niveau liegen, sind die Entstehungsorte der Partikel im Brennraum völlig verschieden. Im Fall der vorgelagerten Einspritzung sind die Partikelemissionen der, bereits aus Kapitel 4.2.1 bekannten, Partikelquelle Einlassventil bzw. Einlassventilsitz zuzuordnen. Bei einer späteren, saugsynchronen Einspritzung verlagert sich die Partikelentstehung an die Zylinderlaufbahn unterhalb der Auslassventile. Wie in einer zeitlich detaillierteren Darstellung in Abbildung A.15 im Anhang ersichtlich ist, fliegen die Tropfen an den offenen Einlassventilen vorbei in Richtung Brennraummitte und somit in Richtung der gegenüberliegenden Zylinderlaufbahn (Bild b-oVI). Da im Bereich der Einlassventile in diesem Zyklus nur eine sehr schwach ausgeprägte Diffusionsflamme zu sehen ist, können zwar offenbar die Wandfilme an den Einlassventilen reduziert werden. Dafür kommt es zu einer kritischen Akkumulation des saugsynchron eingespritzten Kraftstoffes an der Laufbahn. Dieser Partikelentstehungsort liegt außerhalb des Sichtfeldes des Brennraum-Endoskops. Am Transparentaggregat ist dieser Bereich mit Perspektive "1-L" jedoch gut einsehbar, wie Abbildung 4.13 zeigt.

In Form einer Gegenüberstellung der beiden Einspritzstrategien vorgelagert (links) und saugsynchron (rechts) sind die Auftretenswahrscheinlichkeiten für Wandfilme des 

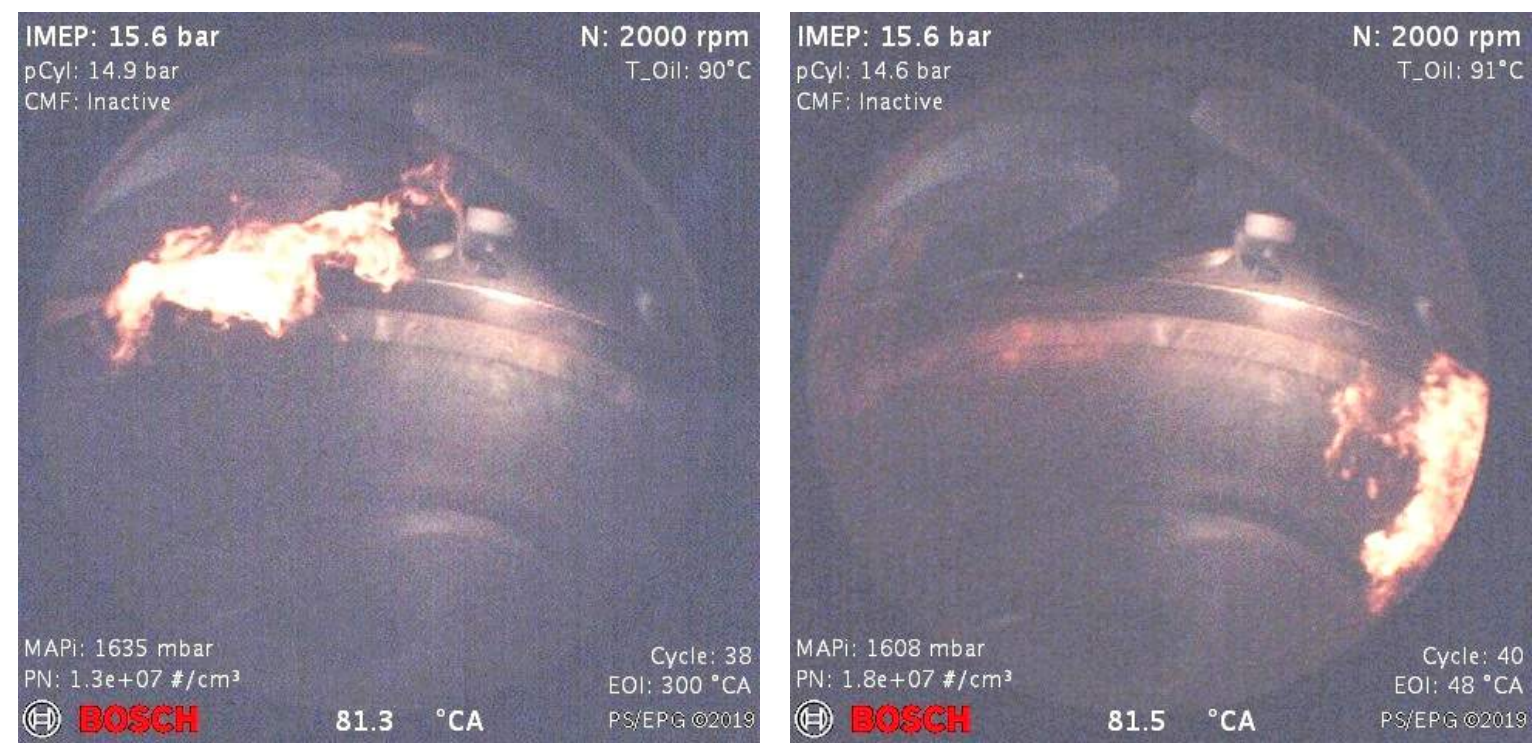

Abbildung 4.12.: Gegenüberstellung von Diffusionsflammen bei $N=2000 \mathrm{~min}^{-1}$, $\mathrm{IMEP}=15,6$ bar und vorgelagertem Timing $\mathrm{EOI}=158^{\circ} \mathrm{KW}$ v. LW-OT bzw. EOI $=300^{\circ} \mathrm{KWv}$. EVs (links) und saugsynchronem EinspritzTiming EOI $=-84^{\circ} \mathrm{KWv}$. LW-OT bzw. EOI $=48^{\circ} \mathrm{KWv}$. EVs (rechts) mit Injektor B

verwendeten Injektorsprays B dargestellt. Perspektive „1-V“ zeigt die Unterseite der Einlassventile. Die Zylinderlaufbahn unterhalb der Auslassventile ist mit Perspektive „1-L" zu sehen. Die seitliche Zylinderlaufbahn zwischen Einlass- und Auslassventilen wird mit Perspektive „2-L“ erfasst. Obwohl am Transparentmotor ein geringerer Saugrohrdruck anliegt als am oben beschriebenen Betriebspunkt der HSVE-Analyse, soll der Einfluss des Einspritztimings auf die Wandfilmbildung anhand dieser Messdaten bewertet werden. Es gilt zu berücksichtigen, dass die detektierten Wandfilmflächen am Transparentmotor aufgrund der deutlich kälteren Brennraumoberflächen ohnehin tendenziell größer ausfallen als am gefeuerten und betriebswarmen Vollmotor. Eine Erhöhung von Saugrohrdruck und eingespritzter Kraftstoffmasse würde zu einer weiteren Verstärkung der hier dargestellten Effekte führen.

Bei vorgelagerter Einspritzung (cVI) ist ein ausgeprägter Wandfilm, hauptsächlich an der Innen- und Unterseite der Einlassventile, erkennbar. Auf der Auslass-seitigen Zylinderlaufbahn sind lediglich einzelne „Spritzer" mit geringer Auftretenswahrscheinlichkeit dokumentiert. Im Fall der saugsynchronen Einspritzung (oVI) fällt sowohl eine geringere Wandfilmfläche an den Einlassventilen als auch eine Verschiebung der benetzten Fläche nach oben auf. Letzteres ist mit einer Ablenkung des Sprays durch die Ansaugströmung erklärbar, welche bei vorgelagerter Einspritzung in ruhendes Gas nicht auftritt. Perspektive „1-L“ offenbart mit oVI einen großflächigen, zusammenhängenden Kraftstoffwandfilm an der Laufbahn unterhalb der Auslassventile, welcher die wesentliche Quelle für Diffusionsflammen und folglich Partikelemissionen der saugsyn- 

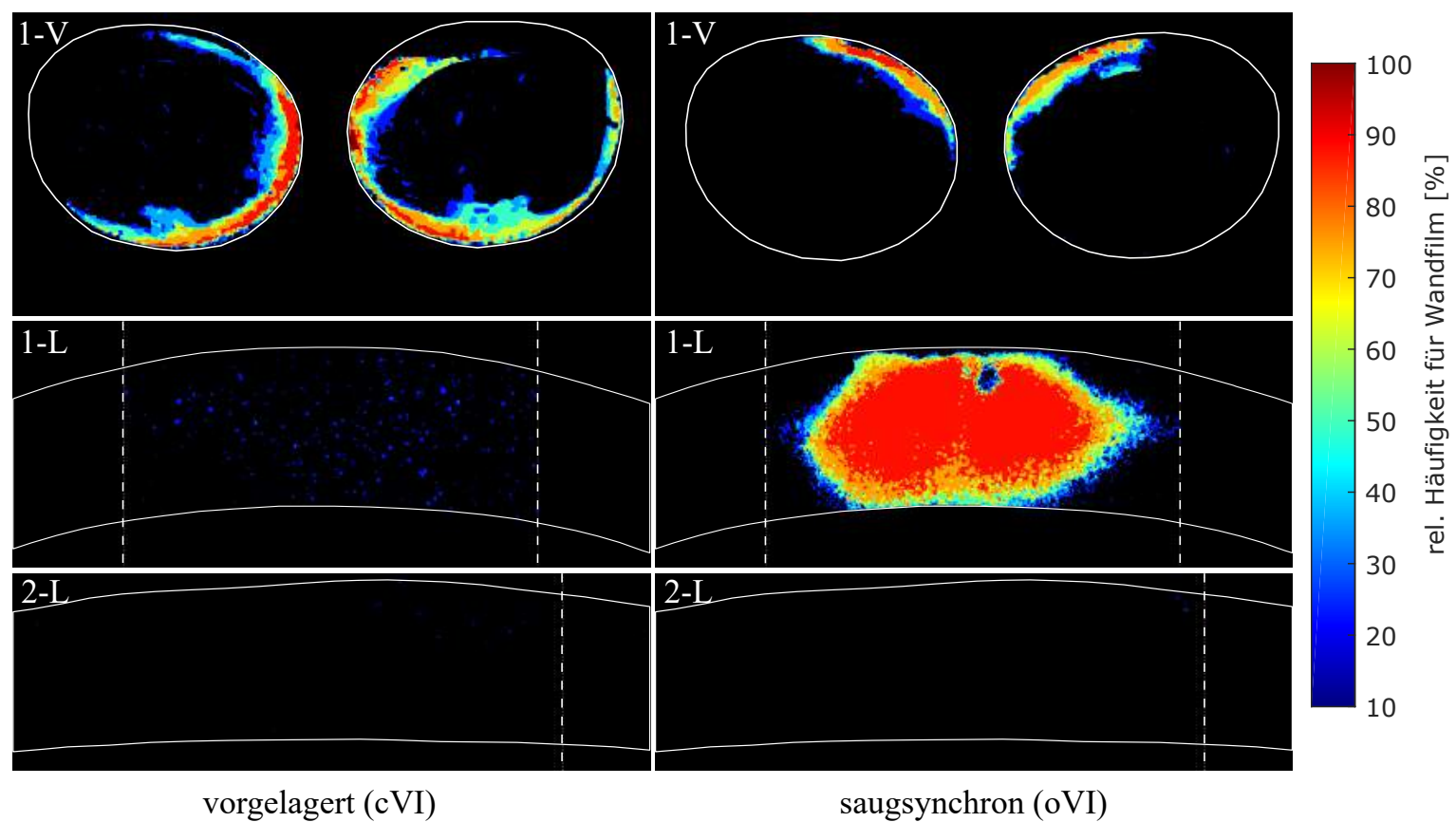

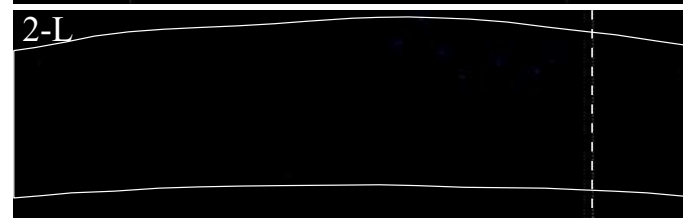

vorgelagert (cVI)
Abbildung 4.13.: Gegenüberstellung der Auftretenswahrscheinlichkeit von Wandfilm nach 10 Zyklen bei vorgelagerter $\left(\mathrm{EOI}=133^{\circ} \mathrm{KW}\right.$ v. LW-OT) und saugsynchroner Einspritzung $\left(\mathrm{EOI}=-103^{\circ} \mathrm{KW}\right.$ v. LW-OT) mit Injektor B und $p_{S R}=900$ mbar am Transparentmotor

chronen Einspritzung darstellt. Mit Injektor B sind für beide Einspritzstrategien nur unwesentliche Benetzungen auf der seitlichen Zylinderlaufbahn (Perspektive „2-L“) zu verzeichnen.

Es stellt sich nun die Frage, mit welchen Maßnahmen dieser extremen Wandfilmbildung bei saugsynchroner Einspritzung entgegengewirkt werden kann. Ein Ansatz mit erhöhter Ladungsbewegung wird in Kapitel 4.2.5 diskutiert. Eine weitere Möglichkeit eröffnet sich durch die Optimierung des Spray-Targetings, auf welche in Kapitel 4.2.6 näher eingegangen wird. Der Einfluss des Einspritztimings auf die Partikelemissionen wurde nun eingehend beleuchtet. Im Folgenden wird auf die Auswirkungen des Einspritzzeitpunktes auf weitere Parameter wie beispielsweise Kraftstoffverbrauch und gasförmige Schadstoffemissionen eingegangen.

Abbildung 4.14 zeigt eine Variation des Einspritzzeitpunktes bei IMEP = 15,6 bar. Die Konzentration der unverbrannten Kohlenwasserstoffe (HC) weist ein ähnlich gelagertes Minimum auf wie die Partikelkonzentration in Abbildung 4.11 bei derselben Last, nämlich kurz nach dem Öffnen der Einlassventile. Auch der Kurvenverlauf ähnelt sich erkennbar. Dies ist verständlich, da Kraftstoffwandfilme im Brennraum, welche zu PN-Emissionen führen, auch HC-Emissionen zur Folge haben können. Außerdem ergibt sich sowohl für die HC- als auch für die PN-Emissionen ein geringer Vorteil durch eine sehr frühe Vorlagerung. Liegt das Einspritzende beispielsweise schon bei $560^{\circ} \mathrm{KW}$ v. LW-OT anstelle von $160^{\circ} \mathrm{KW}$ v. LW-OT stehen bei $N=2000 \mathrm{~min}^{-1}$ etwa 


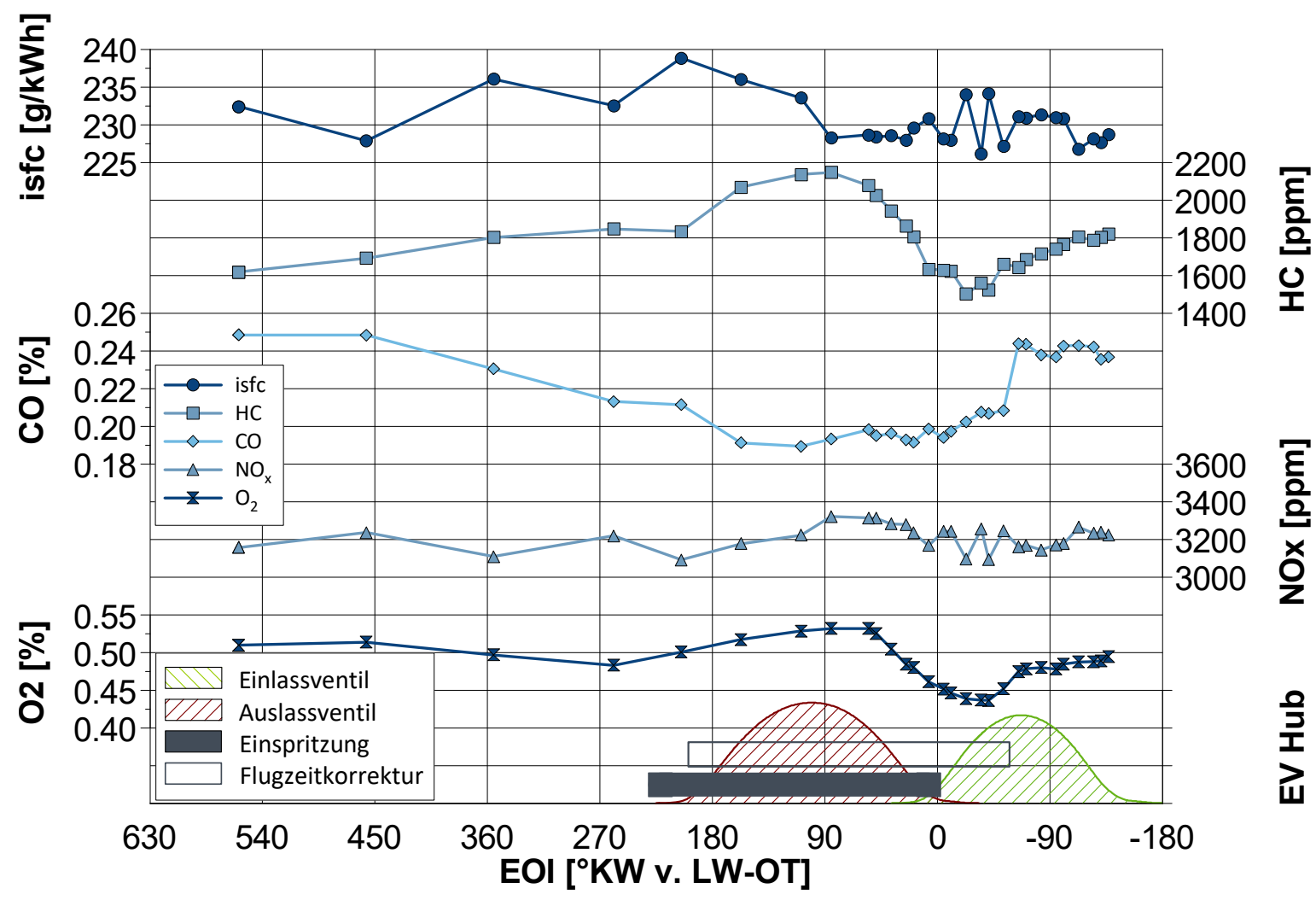

Abbildung 4.14.: Spezifischer Kraftstoffverbrauch und gasförmige Schadstoffemissionen in Abhängigkeit des Einspritztimings bei $N=2000 \mathrm{~min}^{-1}$, $\mathrm{IMEP}=15,6$ bar mit Injektor B

33 ms mehr Zeit für die Kraftstoffverdunstung zur Verfügung. Dass dies eine durchaus relevante Zeitskala für die Kraftstoffverdunstung darstellt, zeigen die Messungen an der Verdunstungskammer (siehe Kapitel 4.1.1).

Die Konzentration der Kohlenstoffmonoxid-Emissionen (CO) weist einen markanten Anstieg bei $-60^{\circ} \mathrm{KW}$ v. LW-OT auf. Für noch spätere EOI ist, wie oben beschrieben, eine starke Wandfilmbildung an der Zylinderlaufbahn zu erwarten. Da es dort lokal zu fetten Gemischzonen kommt, also Sauerstoffmangel vorherrscht, kann weniger $C O \mathrm{zu}$ $\mathrm{CO}_{2}$ reagieren. Darüber hinaus bleibt festzuhalten, dass global betrachtet die Unterschiede der CO-Emissionen recht gering ausfallen und allgemein auf niedrigem Niveau liegen $(0,19-0,25 \%)$.

Die Stickoxid-Emissionen $\left(N O_{x}\right)$ bleiben auf konstantem Niveau und sind bei dieser Untersuchung somit weitestgehend unabhängig vom Einspritztiming. Der bereits erwähnte Restsauerstoff $\left(\mathrm{O}_{2}\right)$ verhält sich ähnlich wie die HC- und PN-Emissionen und erreicht im selben Zeitfenster der öffnenden Einlassventile das Minimum. Dies legt nahe, dass auch die Homogenisierung des Luft-Kraftstoff-Gemisches von der Gasdynamik des Ventilöffnungsvorgangs profitiert. 
Der spezifische Kraftstoffverbrauch ist maßgeblich durch die Verbrennungsschwerpunktlage geprägt, da aufgrund von ottomotorischem Klopfen eine Zündwinkelspätverstellung notwendig ist. Der Motorbetrieb nahe an der Klopfgrenze an diesem Lastpunkt führt daher zu verhältnismäßig späten Schwerpunktlagen im Bereich von $22^{\circ}$ - $27^{\circ} \mathrm{KW}$ n.Z-OT, welche einer gewissen Streuung unterliegen. Weitere Einflüsse auf den Kraftstoffverbrauch, wie beispielsweise aufgrund der HC- oder CO-Emissionen, werden hiervon überlagert.

\subsubsection{Motortemperatur und Transientverhalten}

Bei kaltem Motor fällt es besonders schwer, die Voraussetzungen zu schaffen, die für eine effiziente und schadstoffarme Verbrennung notwendig sind. Da aber gerade der Warmlauf des Motors nach dem Kaltstart bei der Einhaltung von Emissionsgrenzwerten eine entscheidende Rolle spielt (siehe Abbildung A.12), soll der Einfluss der Motortemperatur auf die PN-Emissionen mit Saugrohreinspritzung im Folgenden untersucht werden. Bei ersten Versuchen hat sich gezeigt, dass die Partikelentstehung im Brennraum bei kaltem Motor mitunter so gravierend ist, dass die optischen Zugänge der Endoskope sehr stark verschmutzen. Innerhalb weniger Sekunden sind somit keine optischen Messungen mehr möglich. Daher wurde eine Messmethode entwickelt, bei der mit zwangsgekühltem Motor die Last sprungartig verändert wird.

Die Vorgehensweise dieser Messmethode ist in Abbildung 4.15 beschrieben. Bei einer konstanten Motordrehzahl von $\mathrm{N}=2000 \mathrm{~min}^{-1}$ wird eine Messung stets mit einer initialen Motorlast von $I M E P_{1}=2$ bar gestartet. Nach einer definierten Haltedauer von 180 s erfolgt ein Sprung auf $I M E P_{2}=6,10$ oder 12 bar. Diese Ziellast wird

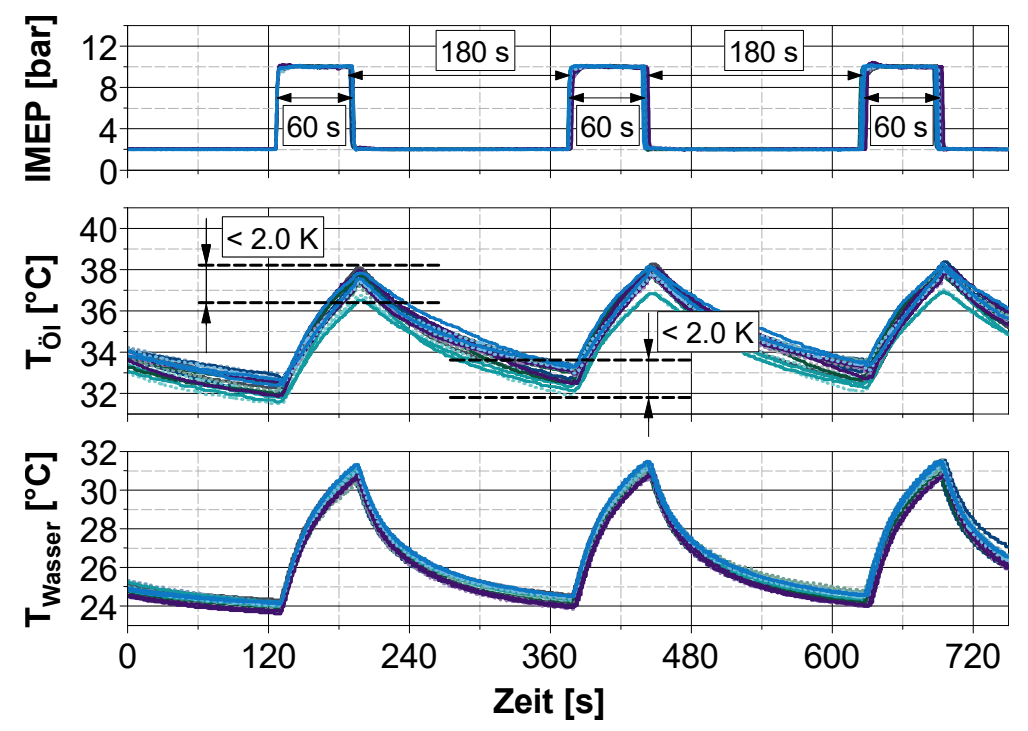

Abbildung 4.15.: Vorgehensweise zur reproduzierbaren Messung von Lastsprüngen bei zwangsgekühltem Motor 
dann für $60 \mathrm{~s}$ gehalten, bevor der negative Lastsprung zurück auf die Ausgangslast $I M E P_{1}=2$ bar eingeleitet wird. Sämtliche weitere Motorparameter wie Steuerzeiten, Stellung der Ladungsbewegungsklappe und Einspritztiming werden während der Lastsprünge konstant gehalten. Lediglich der Zündwinkel wird bei Bedarf angepasst. Die strikte Einhaltung der Wartezeiten zwischen den Lastsprüngen ist wesentlich für die Qualität der Messdaten, da sich andernfalls die Ausgangstemperaturen von Öl und Wasser nicht einstellen können. Die Programmierung eines Skripts für die Automatisierungssoftware RBAS führt zu einem Messablauf mit streng definierter zeitlicher Abfolge und ermöglicht eine sehr gute Reproduzierbarkeit der Messungen, sowohl hinsichtlich der Motortemperaturen, als auch im Hinblick auf die Betriebspunktänderungen. Jeder Lastsprung wird drei mal wiederholt und sämtliche Messdaten im Anschluss zeitlich gemittelt.

Da bei sprungartigen Lastvariationen auch hinsichtlich der Partikelkonzentration starke Gradienten zu erwarten sind, wird für diese Versuche ein DMS500 Partikelzähler zusätzlich zum SPCS2100 installiert. Hintergrund ist das in Kapitel 3.3.3 beschriebene, bessere Ansprechverhalten dieses Messgerätes. Aufgrund der verbleibenden Zweifel hinsichtlich der Abscheidungsfähigkeit von flüchtigen Partikel durch dessen Probenahmesystem, wird lediglich der sogenannte "Accumulation-Mode“ ausgewertet. Die im „Nucleation-Mode" erfassten Kleinstpartikel werden so bewusst ausgeschlossen, um zu vermeiden, dass flüchtige Tröpfchen als vermeintlich feste Partikel gezählt werden.

Der Einfluss der Motortemperatur auf die Partikelemissionen bei vorgelagerter Einspritzung ist in Abbildung 4.16 dargestellt. Auf der linken Bildhälfte befinden sich die Auswertungen bei betriebswarmem Motor, also einer Öltemperatur von $T_{\ddot{O} l}=93^{\circ} \mathrm{C}$. Rechts sind die Ergebnisse bei zwangsgekühltem Motor, also einer mittleren Öltemperatur von $T_{\ddot{\mathrm{O}} l}=35^{\circ} \mathrm{C}$ zu sehen. Bei den kräftigen Linien handelt es sich jeweils um die Mittelwerte der drei Lastsprungwiederholungen, welche in dünnen Linien eingezeichnet sind. Bei der Ausgangslast von $I M E P_{1}=2$ bar ist die PN-Konzentration bei warmem und kaltem Motor mit Werten von $10^{4} \# / \mathrm{cm}^{3}$ und darunter sehr gering und kaum vom Grundrauschen des DMS500 zu unterscheiden. Ähnlich geringe Partikelemissionen werden bei warmem Motor und der geringsten Ziellast von $I M E P_{2}=6$ bar dokumentiert. Das Rauschen des DMS500-Signals bei PN-Konzentrationen $<10^{4} \# / \mathrm{cm}^{3}$ bei warmem Motor und $<10^{5} \# / \mathrm{cm}^{3}$ bei kaltem Motor ist auf einen Kompromiss der vorab zu wählenden Verdünnung zurückzuführen. Damit das Messgerät auch bei hoher Last und hohen PN-Konzentrationen nicht aufgrund von Verschmutzung beschädigt wird, muss zu Beginn der Messungen eine entsprechend hohe Verdünnung eingestellt werden.

In diesem Kontext sei das enorme Spektrum der erfassten PN-Konzentrationen hervorgehoben, welches sich über vier Größenordnungen erstreckt. Es besteht eine sehr starke Sensitivität der Partikelemissionen gegenüber der Motortemperatur. Selbst bei Lastsprüngen auf die niedrigste Ziellast von $I M E P_{2}=6$ bar ergibt sich eine Erhöhung der gemessenen Partikelkonzentration bei Zwangskühlung des Motor um den Faktor 1000. Vergleicht man die gemittelten Lastsprünge auf die höchste Ziellast von 

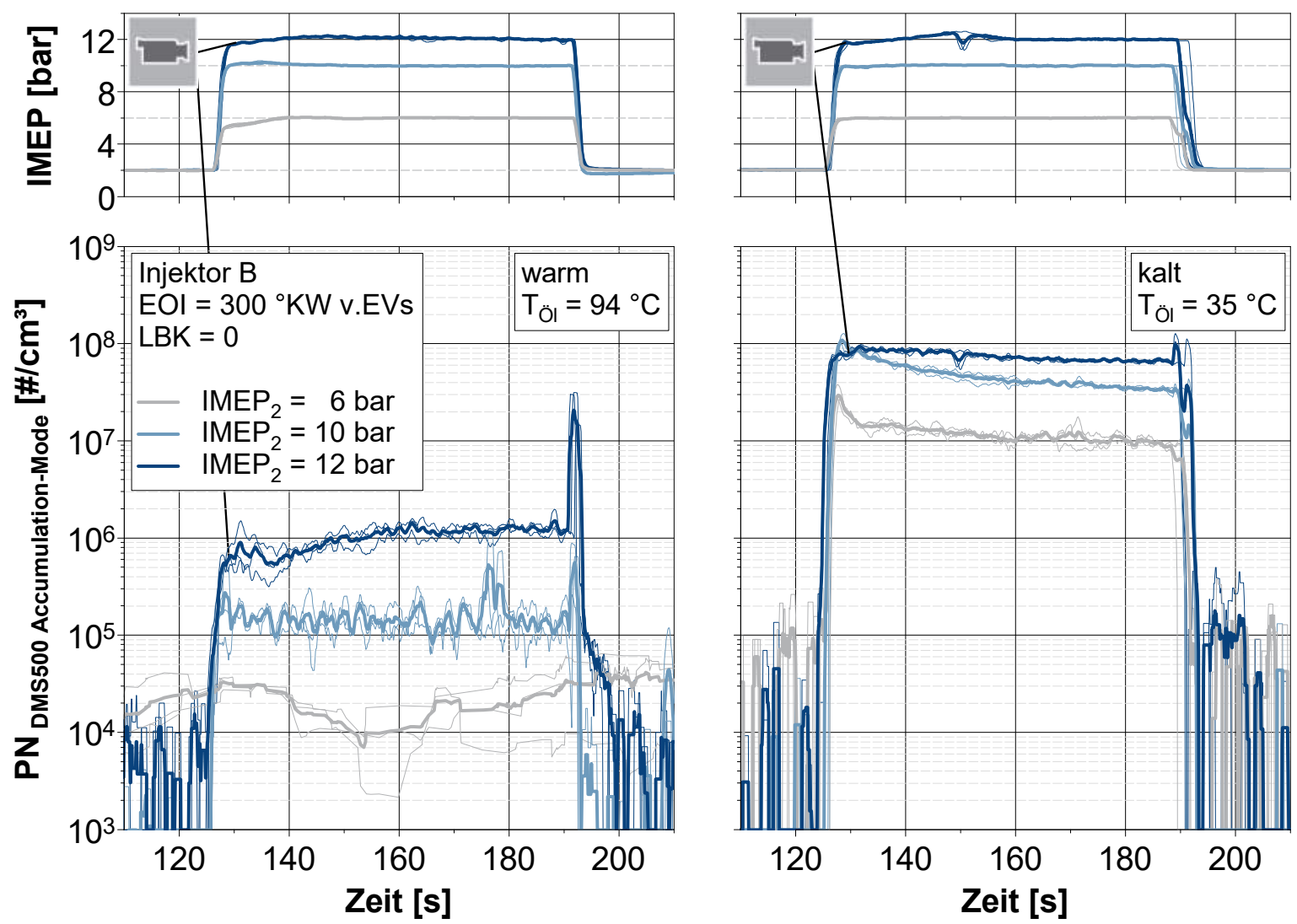

Abbildung 4.16.: Vergleich der mittleren PN-Emissionen aus jeweils drei Lastsprüngen von $I M E P_{1}$ auf $I M E P_{2}$ bei warmem Motor mit $T_{\ddot{\mathrm{O}} l}=94^{\circ} \mathrm{C}$ (links) und kaltem Motor mit $T_{\ddot{\mathrm{O}} l}=35^{\circ} \mathrm{C}$ (rechts)

$I M E P_{2}=12$ bar ist bei kaltem Motor ein Anstieg um den Faktor 100 zu verzeichnen. Die PN-Spitze, welche bei Einleitung des negativen Lastsprungs auftreten kann, ist auf eine kurzzeitige Gemischanfettung zurückzuführen. Dies ist durch eine Kalibrierung der Funktion „Übergangskompensation“ im Motorsteuergerät vermeidbar , welche für die hier beschriebenen Versuche jedoch bewusst deaktiviert wurde. Der hohe Zeitaufwand für die Applikation dieser Funktionalität für die hier und im Folgenden untersuchten prototypischen Injektorsprays bei verschiedenen Lastvariationen und Motortemperaturen steht in keinem guten Verhältnis zum überschaubaren Nutzen im Rahmen dieser Studie.

Um die ausgeprägte Partikelentstehung bei kaltem Motor besser zu verstehen, erfolgt eine Gegenüberstellung der endoskopisch erfassten Diffusionsflammen im Brennraum in Abbildung 4.17. Die dargestellten Verbrennungen werden kurz nach Erreichen der Ziellast von $I M E P_{2}=12$ bar aufgezeichnet. Die entsprechenden Zeitpunkte während des Lastsprunges sind zum besseren Verständnis in Abbildung 4.16 mit Kamerasymbolen markiert. Es ist unschwer zu erkennen, dass bei kaltem Motor deutlich intensivere Diffusionsflammen auftreten als bei warmem Motor. Bei kaltem Motor sind auch kleinere Diffusionsflammen am oberen Ende der Zylinderlaufbahn zu sehen, der 

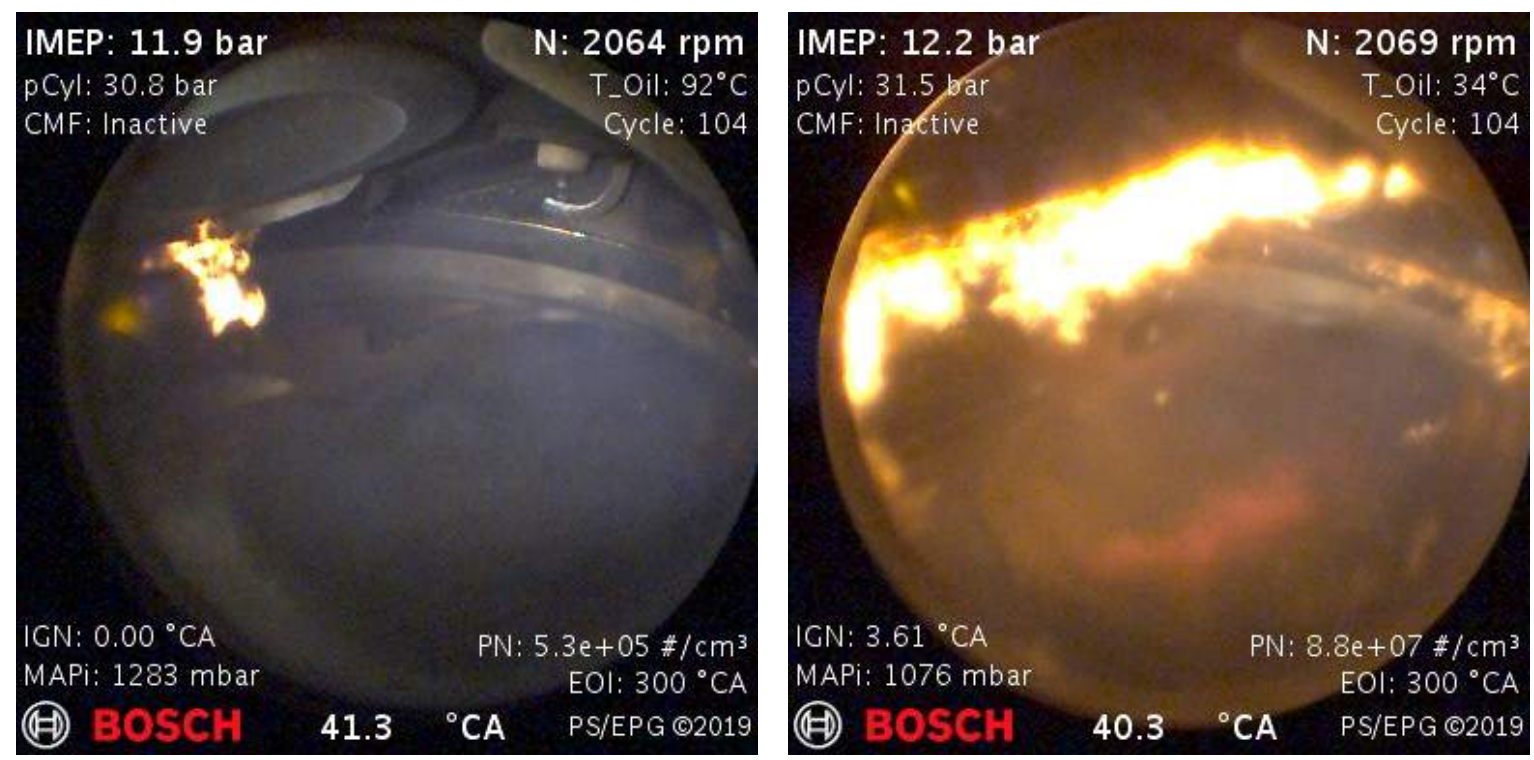

Abbildung 4.17.: Gegenüberstellung von Verbrennungen mittels HSVE mit $N=2000 \mathrm{~min}^{-1}$ bei Erreichung der Ziellast von $I M E P_{2}=12$ bar mit warmem Motor (links) und kaltem Motor (rechts)

Hauptentstehungsort der Partikelemissionen ist jedoch weiterhin der Bereich im Umfeld der Einlassventilsitze.

Die in den vorangegangenen Kapiteln beschriebenen Kraftstoffwandfilme können folglich bei kälterer Motortemperatur deutlich langsamer verdunsten. Dies geht auch aus den Versuchen in der Verdunstungskammer in Kapitel 4.1.1 hervor. Dies hat zur Folge, dass mehr flüssiger Kraftstoff zum Zündzeitpunkt im Brennraum vorliegt, welcher die gezeigten, intensiven Diffusionsflammen verursacht.

An dieser Stelle muss weiterhin berücksichtigt werden, dass der hier anhand einer Zwangskühlung im stationären Betrieb eingestellte Zustand „kalter Motor“ die Realität eines kalten Motors im Warmlauf, also direkt nach einen Kaltstart, nur unzureichend abbilden kann. Zwar gelingt es reproduzierbar die Kühlmittel- und Öltemperatur auf niedrigem Niveau zu halten, die Temperaturen der Einlassventile sowie der Kanal- und Brennraumwände sind jedoch sicherlich höher als bei einem tatsächlichen Motorwarmlauf. Ein solches Fahrmanöver während des Motorwarmlaufs, direkt nach einem Kaltstart, mit einer Laständerung von $I M E P_{1}=2$ bar nach $I M E P_{2}=12$ bar, hätte demnach noch deutlich höhere Partikelkonzentrationen zur Folge als bei den hier vorgestellten Messungen. 


\subsubsection{Ladungsbewegung}

Wie in Abbildung 3.8 in Kapitel 3.3.1 dargestellt, besteht an den verwendeten Versuchsträgern, sowohl am Vollmotor als auch am Transparentaggregat, die Möglichkeit eine Ladungsbewegungsklappe (LBK) im Saugrohr anzustellen. Dadurch kann eine sogenannte „Tumble“-Strömung, also ein Wirbel mit Rotationsachse parallel zur Kurbelwelle, induziert beziehungsweise verstärkt werden. Die Klappe befindet sich stromabwärts am Ende des Saugrohrs, und somit unmittelbar am Übergang zum Einlasskanal im Zylinderkopf.

In diesem Teilkapitel soll analysiert werden, inwiefern eine veränderte Ladungsbewegung, genauer eine verstärkte Tumble-Strömung, die Gemischbildung hinsichtlich der Partikelemissionen beeinflussen kann. Wie im vorangegangenen Unterkapitel erörtert, ist es insbesondere bei kaltem Motor notwendig, Maßnahmen zur Partikelreduktion zu finden. Daher soll im folgenden das Potenzial einer aktivierten LBK an einem Betriebspunkt mit hoher Last, saugsynchronem Einspritztiming (oVI) und kaltem Motoröl bzw. Kühlwasser untersucht werden. Um bei der Bewertung der Ladungsbewegung dem Einfluss zyklischer Schwankungen gerecht zu werden, wird an dieser Stelle auf die statistische Auftretenswahrscheinlichkeit von Diffusionsflammen zurückgegriffen, anstelle von exemplarischen Beispielen einzelner Zyklen. Die Methode zur Herleitung dieser Darstellung ist in Kapitel 3.3.2 beschrieben. Abbildung 4.18 zeigt eine Gegen-

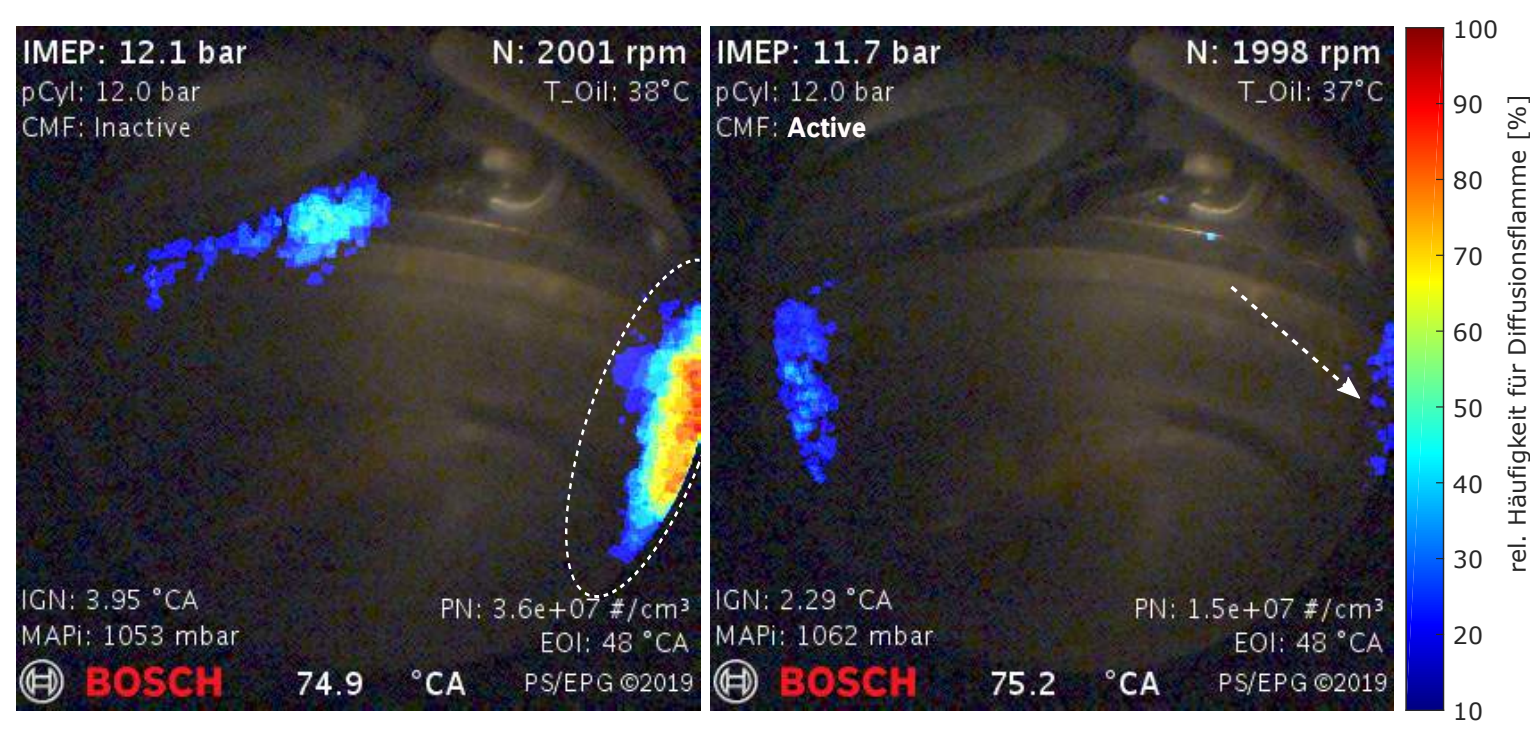

Abbildung 4.18.: Gegenüberstellung der räumlichen Auftretenswahrscheinlichkeit von Diffusionsflammen mittels HSVE mit $N=2000 \mathrm{~min}^{-1}$ bei Erreichung der Ziellast von $I M E P_{2}=12$ bar mit deaktivierter LBK (links) und aktivierter LBK (rechts) mit Injektor B

überstellung zweier statistischer Analysen mittels HSVE an diesem Betriebspunkt mit aktivierter Ladungsbewegungsklappe rechts und deaktivierter LBK links. Zwar ist die Partikelkonzentration mit $1,5 \cdot 10^{7} \# / \mathrm{cm}^{3}$ mit aktivierter LBK immer noch sehr hoch, 
doch kann sie gegenüber dem Ausgangszustand ohne Tumble-Verstärkung in etwa halbiert werden. Weiterhin können Diffusionsflammen an der Zylinderlaufbahn unterhalb der Auslassventile erheblich reduziert werden (weißer Pfeil ggü. weiße Elipse). Außerdem fällt auf, dass an den Einlassventilsitzen praktisch keine Partikel mehr entstehen. Allerdings ist eine Verlagerung dieser Partikelquelle an die äußere Zylinderlaufbahn, seitlich des Einlassventils, zu beobachten.

Die beschriebenen Veränderungen hinsichtlich der Diffusionsflammen durch Anstellung der Ladungsbewegungsklappe sollen im Folgenden anhand von Wandfilm-Analysen am Transparentmotor genauer untersucht werden, um die Auswirkungen der TumbleVerstärkung auf die Gemischbildung besser zu verstehen. Dazu wird der oben beschriebene Betriebspunkt am Transparentmotor nachgestellt und jeweils mit aktivierter und deaktivierter LBK analysiert, wie Abbildung 4.19 zeigt. Auf den ersten Blick sind mit
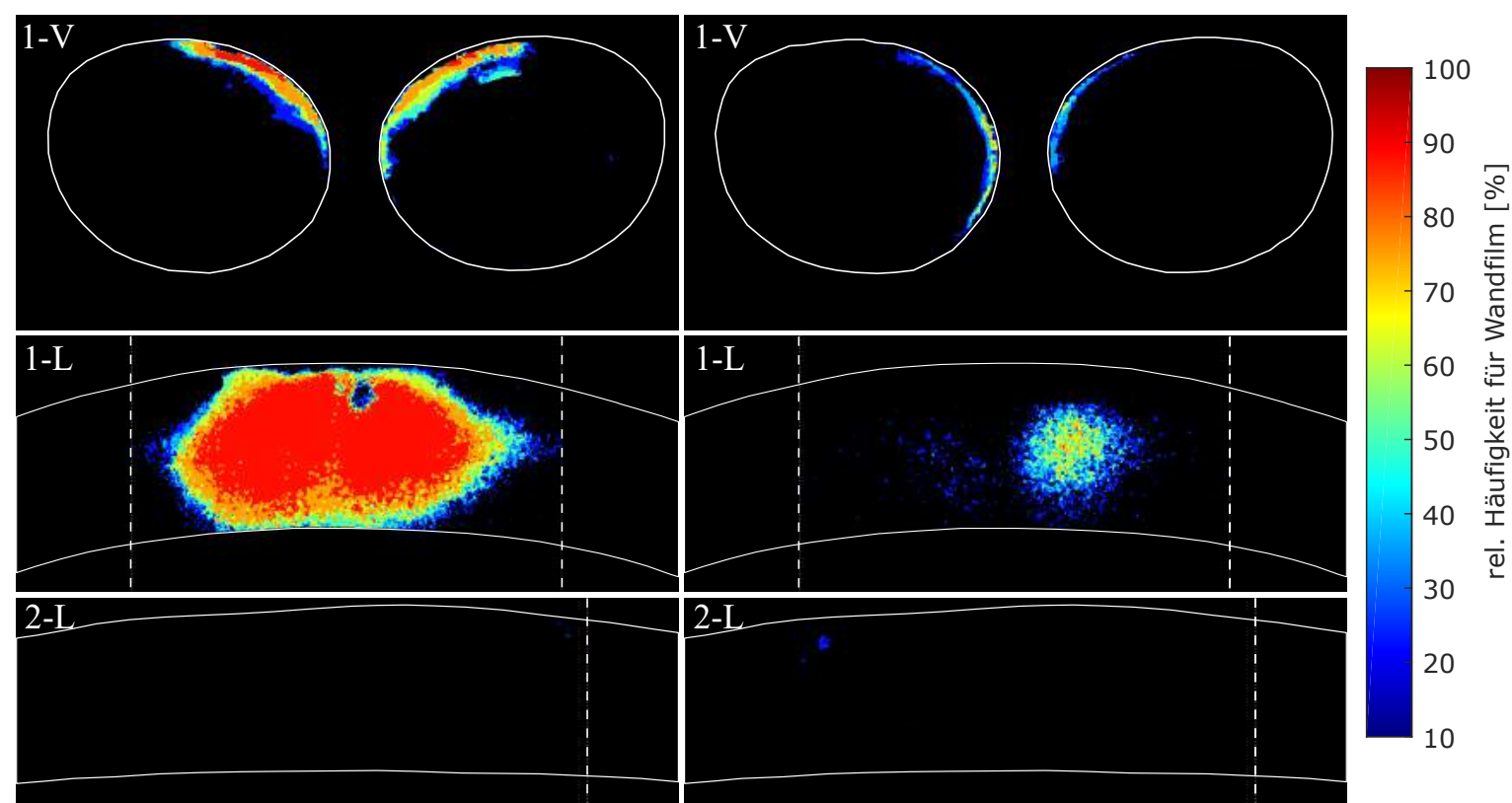

oVI \& inaktive LBK

oVI \& aktive LBK

Abbildung 4.19.: Gegenüberstellung der Auftretenswahrscheinlichkeit von Wandfilm nach 10 Zyklen bei saugsynchroner Einspritzung (oVI) mit deaktivierter LBK (links) und aktiver LBK (rechts) mit Injektor B

aktiver LBK sowohl an den Einlassventilen (Perspektive „1-V“) als auch an der Zylinderlaufbahn unterhalb der Auslassventile (Perspektive „1-L“) deutlich reduzierte Wandfilmflächen erkennbar. Auch am Transparentmotor ist, wenn auch in geringerer Ausprägung als am gefeuerten Vollmotor, eine Wandfilmbildung an der äußeren Zylinderlaufbahn mit Perspektive „2-L“ bei Tumble-Verstärkung festzustellen.

Es stellt sich nun die Frage nach dem offenbar sehr effektiven Wirkmechanismus zur Reduktion von Kraftstoffwandfilmen im Brennraum durch erhöhte Ladungsbewegung. Ein Bestandteil des Erklärungsmodels besteht darin, dass eine Tumble-Verstärkung, 
insbesondere bei saugsynchroner Einspritzung, die Flugbahn der Spray-Tropfen derart verändert, dass weniger Tropfen direkt an die Zylinderlaufbahn gelangen. Ein weiterer Aspekt ist die Querschnittsverengung der Ansaugströmung durch die Anstellung der Ladungsbewegungsklappe, welche eine erhöhte Strömungsgeschwindigkeit im Einlasskanal zur Folge hat. Nach Himmelsbach [47, Elsäßer [31] und Ebner [29] nimmt die Wandfilmhöhe bei steigender Gasgeschwindigkeit aufgrund der größeren induzierten Schubspannungen ab. Zusätzlich sorgt eine höhere Geschwindigkeit der Ansaugströmung für eine höhere Weber-Zahl, und damit unter Umständen für eine höhere Wahrscheinlichkeit für Sekundärzerstäubung des Kraftstoffsprays. Letzteres soll im Folgenden anhand von überschlägigen Berechnungen überprüft werden.

In Abbildung 4.20 sind die Messdaten, Zwischenergebnisse und berechneten WeberZahlen sowie die kritischen Tropfendurchmesser für zwei Messungen dargestellt. Es handelt sich jeweils um Daten desselben Betriebspunktes bei $N=2000 \mathrm{~min}^{-1}$, IMEP $=12$ bar und saugsynchroner Einspritzung, jedoch ist im zweiten Fall die Ladungsbewegungsklappe aktiviert. Anhand von Ladungswechselrechnungen mit dem Tool BeCat, können die Massenströme über die Einlass- und Auslassventile ermittelt werden. Da die geometrischen Querschnittsflächen des Einlasskanals, sowohl bei offener, als auch bei angestellter Ladungsbewegungsklappe, bekannt sind, kann die Strömungsgeschwindigkeit im Einlasskanal für beide Fälle berechnet werden. Unter Berücksichtigung der Strömungskoeffizienten der Einlassventile wird zum Vergleich auch die Strömungsgeschwindigkeit im Ventilspalt der Einlassventile beziffert. Hierbei wird bereits deutlich, dass im Ventilspalt, unabhängig von der Stellung der Ladungsbewegungsklappe, stets sehr hohe Geschwindigkeiten vorherrschen. Da diese mitunter größer als $c_{\text {mach }}=0,3$ sind, müssen diese Werte unter Vorbehalt betrachtet werden, da dann ein Gas nicht mehr vereinfachend als inkompressibel betrachtet werden darf. Außerdem wird ersichtlich, dass eine Anstellung der Ladungsbewegungsklappe (LBK = 1) die Strömungsgeschwindigkeit im Einlasskanal mehr als verdoppeln kann.

Mit den Annahmen für die Oberflächenspannung von Benzin $\sigma_{K r}=0,02 \mathrm{~kg} / \mathrm{s}^{2}$, die Tropfengeschwindigkeit des Sprays $c_{T r}=20 \mathrm{~m} / \mathrm{s}$ und der charakteristischen Tropfengröße des Sprays $d_{\text {Tr.char. }}=100 \mu m$ kann nun die Weber-Zahl der Tropfen im Einlasskanal und im Ventilspalt anhand von Gleichung 2.3 berechnet werden. Wie in Kapitel 2.2.2 beschrieben, existieren mehrere kritische Weberzahlen für diverse Zerfallsarten. In diesem Fall gilt es jedoch zu klären, ob überhaupt die Bedingungen für Sekundärzerfall vorliegen. Daher wird die kleinste der kritischen Weber-Zahlen $W_{k r i t}=12$ herangezogen. Im Falle der deaktivierten Ladungsbewegungsklappe $(\mathrm{LBK}=0)$ ist die Weber-Zahl im Einlasskanal nahe null. Das liegt an der geringen Relativgeschwindigkeit zwischen Ansaugströmung und Tropfen.

Für den Fall der aktivierten Ladungsbewegungsklappe $(\mathrm{LBK}=1)$ wird die kritische Weber-Zahl zwölf für eine Zeitspanne von über $100^{\circ} \mathrm{KW}$ deutlich überschritten und erreicht einen Maximalwert von $W e=41,6$. Damit kommt es für große Tropfen des Sprays zur vermuteten Sekundärzerstäubung im Einlasskanal. Die Berechnung des kritischen Tropfendurchmessers $d_{k r i t}$ nach Gleichung 2.4 veranschaulicht, dass mit 
LBK $=1$ nicht nur sehr große Tropfen von der Ansaugströmung zerkleinert werden können, sondern auch Tropfen, welche kleiner sind als der mittlere Sauter-Durchmesser $\mathrm{SMD}=70 \mu \mathrm{m}$ des Tropfensprays von Injektor B. Trotz der Einschränkungen hin-
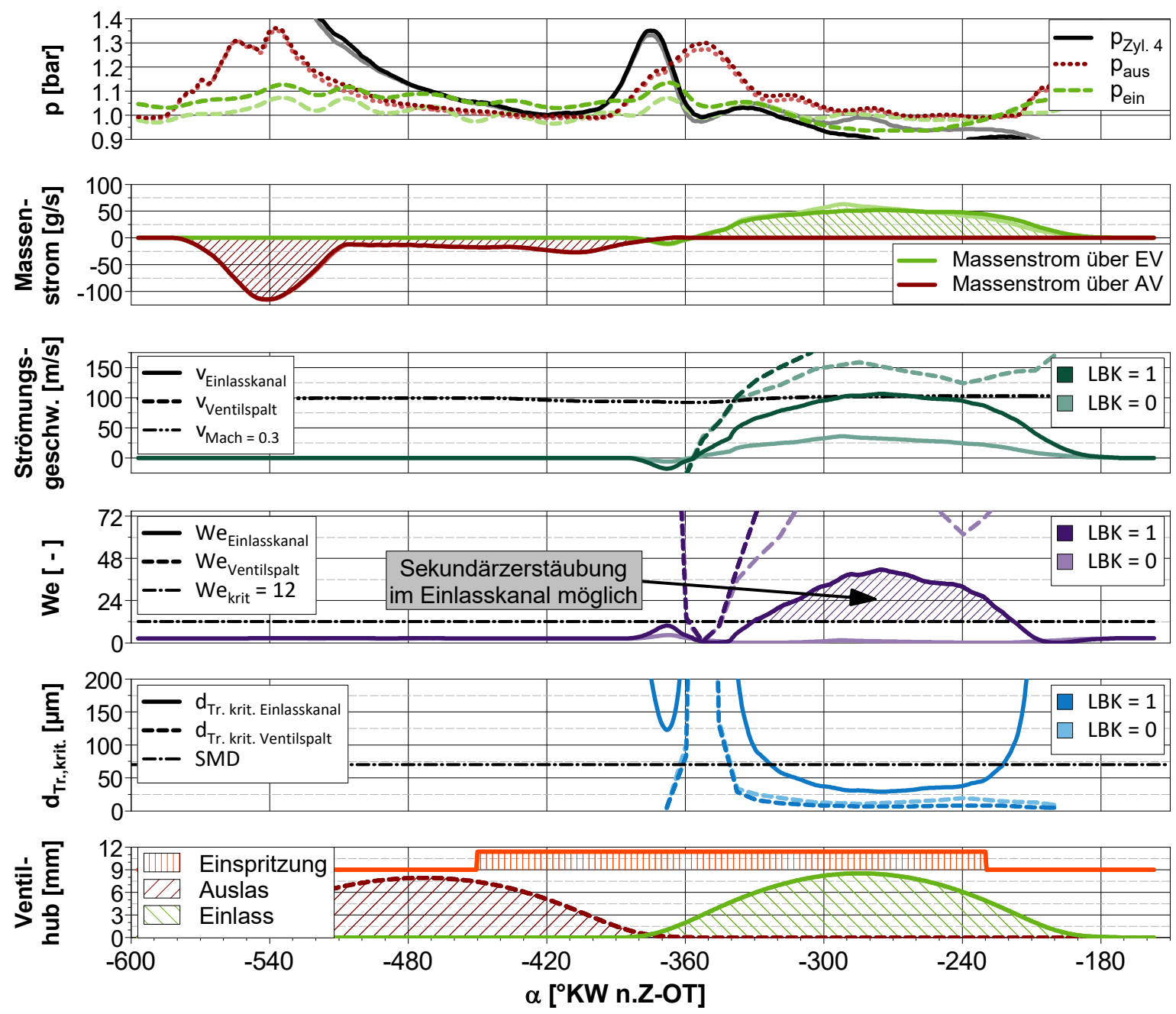

Abbildung 4.20.: Berechnung der Weber-Zahl $W e$ sowie des kritischen Tropfendurchmessers $d_{\text {Tr.krit. }}$ mit Annahmen 3 auf Basis von Ladungswechselrechnungen bei $\mathrm{N}=2000 \mathrm{~min}^{-1}$, IMEP $=12$ bar mit Injektor B

sichtlich der Aussagekraft durch die Überschreitung der Mach-Zahl von 0,3 in weiten Teilen, liefern die Daten zu Weber-Zahl und kritischem Tropfendurchmesser im Ventilspalt Grund zur Annahme, dass im Ventilspalt auch mit deaktivierter Ladungsbewegungsklappe Sekundärzerstäubung möglich ist. Allerdings stellt der Ventilspalt nur einen sehr kurzen Streckenabschnitt der Tropfenflugbahnen von einigen Millimetern dar. Eine hohe Wahrscheinlichkeit für Sekundärzerstäubung im gesamten Einlasska-

${ }^{3}$ Annahmen für die Oberflächenspannung von Benzin $\sigma_{K r}=0,02 \mathrm{~kg} / \mathrm{s}^{2}$, die Tropfengeschwindigkeit des Sprays $v_{T r}=20 \mathrm{~m} / \mathrm{s}$ und der charakteristischen Tropfengröße des Sprays $d_{\text {Tr.char. }}=100 \mu \mathrm{m}$ 
nal im Zylinderkopf auf etwa $100 \mathrm{~mm}$ Länge ist möglicherweise deutlich wirksamer im Hinblick auf die Gemischbildung.

An dieser Stelle sei neben den bereits erwähnten Annahmen auf weitere Unschärfen der oben beschriebenen Berechnungen eingegangen. So wurde beispielsweise die Beschleunigung der Tropfen durch die Ansaugströmung vernachlässigt, wodurch deren Relativgeschwindigkeit, und somit auch die Weber-Zahl, tendenziell überschätzt wird. Die Tatsache, dass die Tropfen von der Injektorspitze ausgehend nicht exakt in Strömungsrichtung gespritzt werden, wurde ebenfalls nicht berücksichtigt. Diese Vereinfachung führt allerdings eher zu einer Unterschätzung der Weber-Zahl, da die Relativgeschwindigkeiten der Tropfen bei Einbeziehung der entsprechenden Geschwindigkeitskomponente der Tropfen in Strömungsrichtung kleiner ausfiele als bei Verwendung des angenommenen Absolutwerts. Weiterhin wird vernachlässigt, dass sich der Strömungsquerschnitt stromabwärts der Ladungsbewegungsklappe allmählich wieder vergrößert, da keine Leitbleche verwendet werden. Da eine direkte Benetzung solcher Leitbleche durch das Kraftstoffspray nur mit starken Einschränkungen hinsichtlich des Spray-Targetings vermeidbar wäre, wird auf diese verzichtet. Außerdem wird von einer über den Kanalquerschnitt konstanten Strömungsgeschwindigkeit ausgegangen. Es wird also kein Strömungsprofil der turbulenten Ansaugströmung hinterlegt. Dies ist zwar insbesondere in Wandnähe unzutreffend, global betrachtet ist jedoch hierdurch eher mit einer geringfügigen Unterschätzung der Weber-Zahl zu rechen, da in der Kanalmitte tatsächlich von höheren Gasgeschwindigkeiten ausgegangen werden kann. Insofern sind die berechneten Werte mit einer gewissen Unsicherheit behaftet. Aufgrund der sehr deutlichen Überschreitung der kritischen Weber-Zahl für Sekundärzerstäubung kann die prinzipielle Aussage der Rechnung jedoch als unzweifelhaft betrachtet werden.

Neben dem diskutierten Potenzial einer Ladungsbewegungsklappe die Gemischbildung zu verbessern und dadurch Kraftstoffwandfilme im Brennraum und letztlich Partikelemissionen zu reduzieren, gilt es aber auch, etwaige Nachteile zu bedenken. So müssen beispielsweise höhere Kosten für die Motorentwicklung und Fertigung in Kauf genommen werden. Außerdem gilt es bei der Entwicklung das Risiko zu minimieren, dass bei einem Defekt Teile der Klappen in den Motor gelangen. Ein weiterer Aspekt sind die steigenden Ladungswechselverluste, welche bei angestellter Ladungsbewegungsklappe zu Buche schlagen. Ob diese tatsächlich zu einem schlechteren spezifischen Verbrauch führen, ist betriebspunktabhängig. Es besteht die Möglichkeit, dass die Verluste im Ladungswechsel durch eine höhere Brenngeschwindigkeit und geringere HC-Emissionen kompensiert werden. Dies ist bei einigen Messungen der vorliegenden Arbeit zu beobachten. Auf eine tiefer gehende Betrachtung dieser Aspekte wird an dieser Stelle jedoch verzichtet.

Bei genauer Analyse der Daten aus der HSVE fällt auf, dass mitunter auch Inhomogenitäten in der Gasphase zu Diffusionsflammen und somit zur Partikelbildung führen können, wie Abbildung 4.21 zeigt. Auf der linken Bildhälfte ist eine einzelne, beispielhafte Verbrennung abgebildet. Die angezeigten Messdaten liegen zum Zeitpunkt der 


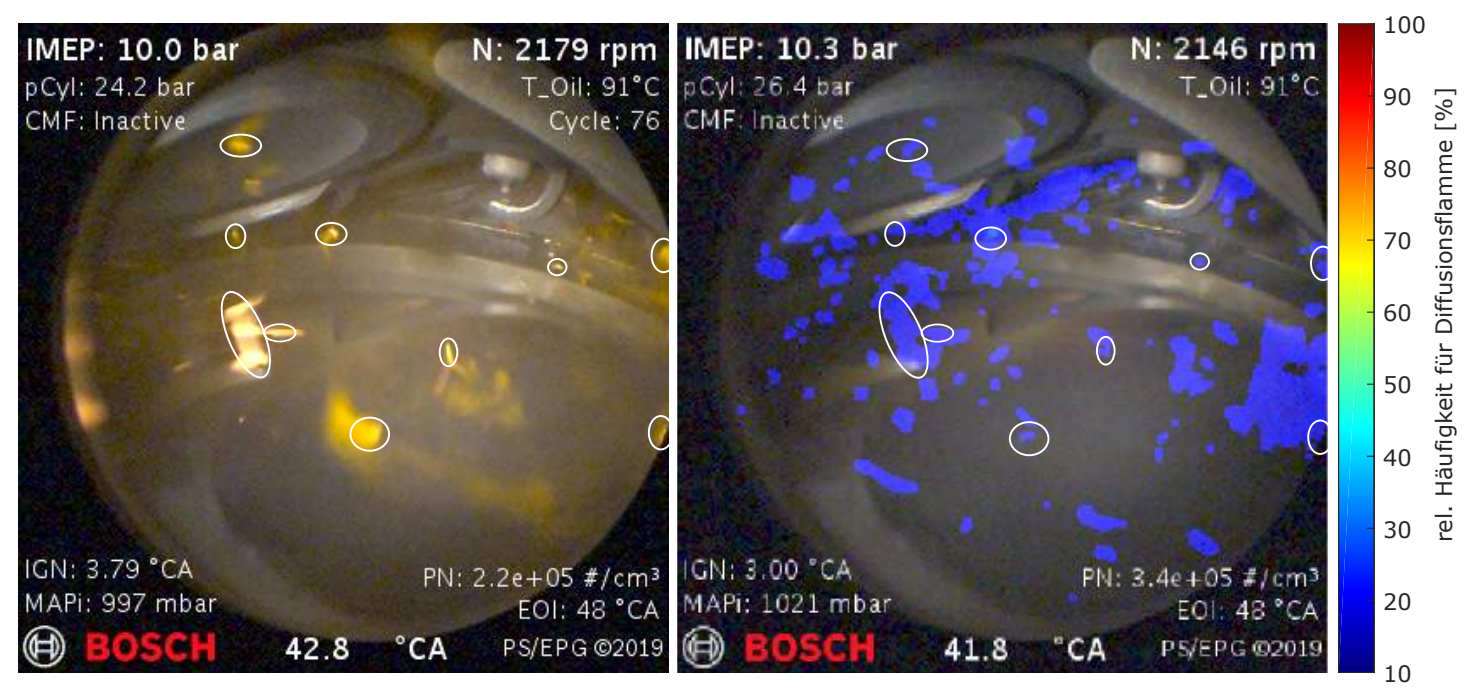

Abbildung 4.21.: Diffussionsflammen eines einzelnen Zyklus (links) und statistische Auswertung über 20 Zyklen (rechts) bei $I M E P_{2}=10$ bar mit Injektor G

Aufnahme dieses Bildes vor. Selbst bei warmem Motor, saugsynchroner Einspritzung und einer moderaten Ziel-Last von $I M E P_{2}=10 \mathrm{bar}$ in Folge eines Lastsprunges, sind mehrere, kleine in der Gasphase verteilte Diffusionsflammen zu erkennen. Eine statistische Auswertung der Auftretenswahrscheinlichkeit von Diffusionsflammen über 20 Zyklen ist auf der rechten Seite dargestellt. Die dort angezeigten Messdaten stellen Mittelwerte dieser Zyklen dar. Anhand der nahezu durchgängig dunkelblauen Einfärbung der Auftretensorte wird deutlich, dass jeweils nur eine einzige Flamme an diesen Stellen im Brennraum dokumentiert wird (relative Häufigkeit $<20 \%$ ). Es

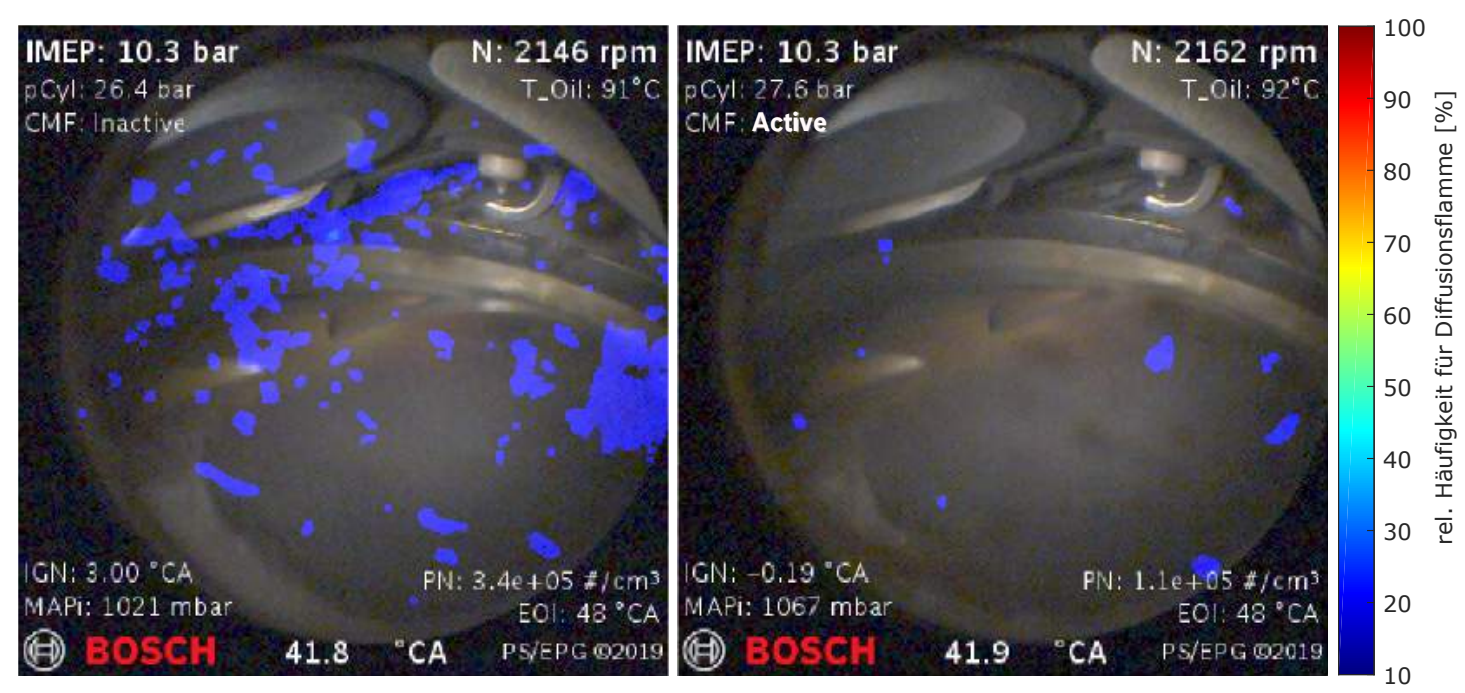

Abbildung 4.22.: Gegenüberstellung der räumlichen Auftretenswahrscheinlichkeit von Diffusionsflammen im Lastbereich von $I M E P_{2}=10$ bar mit deaktivierter LBK (links) und aktivierter LBK (rechts) mit Injektor G 
handelt sich also nicht um eine Akkumulation von Kraftstoff, wie es bei den bislang gezeigten Wandfilmen der Fall war, sondern um einzelne Zonen in der Gasphase mit unter-stöchiometrischem Gemisch, deren Ursprung höchstwahrscheinlich unvollständig verdunstete Kraftstofftropfen sind.

In Abbildung 4.22 wird deutlich, dass eine Erhöhung der Tumble-Strömung mittels einer Ladungsbewegungsklappe nicht nur zu einer reduzierten Bildung von Kraftstoffwandfilmen im Brennraum führen, sondern auch die Homogenisierung des LuftKraftstoff-Gemisches unterstützen kann. Der Vergleich zweier, bis auf die Stellung der LBK, gleich ausgeführten Lastsprünge zeigt deutlich, wie die Wahrscheinlichkeit für Diffusionsflammen in der Gasphase mit erhöhter Ladungsbewegung reduziert werden kann. Auch die gemessene Partikelkonzentration sinkt im Mittel deutlich auf etwa $32 \%$ des Ausgangswertes. Es ist anzunehmen, dass auch in diesem Szenario eine Sekundärzerstäubung der Tropfen im Einlasskanal zu dieser Verbesserung beiträgt. Eine erhöhte Ladungsbewegung im Brennraum nach Beendigung des Ansaugvorgangs unterstützt die Homogenisierung des Gemisches und die Verdunstung verbliebener Tröpfchen zusätzlich.

\subsubsection{Spraywinkel bei Single-Konfiguration}

Im folgenden Teilkapitel soll der Einflussparameter Spraywinkel hinsichtlich seines Optimierungspotenzials für geringere Partikelemissionen untersucht werden. Eine isolierte Betrachtung dieses Parameters, ohne die Berücksichtigung von Motortemperatur, Motorlast, Einspritztiming und Ladungsbewegung, birgt jedoch das Risiko, wesentliche Korrelationen zu übersehen. Daher werden die bereits vorgestellten Einflussparameter mit verschiedenen, neuen Sprayauslegungen kombiniert. Eine Übersicht des dadurch aufgespannten Parameterraums gibt Abbildung 4.23. Von den insgesamt acht verschie-

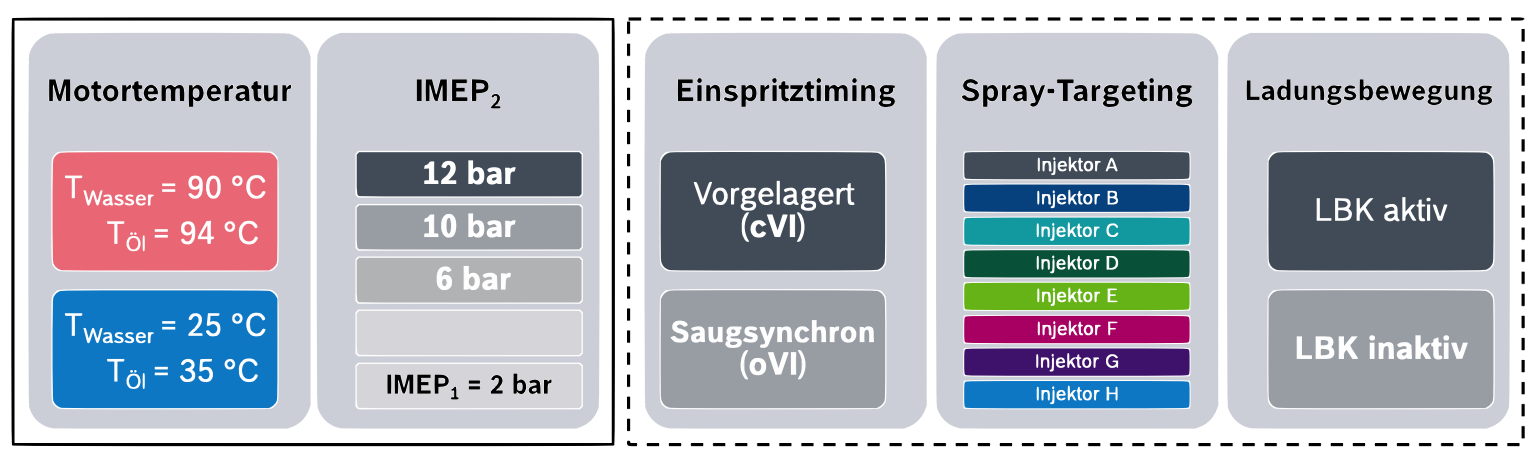

Abbildung 4.23.: Parameterraum Targetingstudie

denen Spray-Targeting Varianten (Injektor A .. H) sollen zugunsten der Übersichtlichkeit jedoch lediglich vier prinzipiell unterschiedliche Auslegungen vorgestellt werden, deren Kenndaten in Tabelle 4.1 zusammengestellt sind. 4 Zum besseren Verständnis

\footnotetext{
${ }^{4}$ Ergebnisse aller untersuchten Injektoren sind in Abbildung A.16 und A.17 im Anhang zu finden.
} 


\begin{tabular}{|c|c|c|c|c|c|}
\hline \multicolumn{2}{|c|}{ Injektor } & B & $\mathrm{D}$ & $\mathbf{E}$ & $\mathrm{G}$ \\
\hline \multicolumn{2}{|c|}{ Variation } & Basis & $\alpha_{50}$ & $\beta_{80}$ & $\beta_{80} \& \mathrm{SMD}$ \\
\hline Тур & {$[-]$} & $\mathrm{E}$ & $\mathrm{E}$ & $\mathrm{E}$ & $\mathrm{E}$ \\
\hline$Q_{\text {stat }}$ & {$[\mathrm{g} / \mathrm{min}]$} & 106 & 106 & 106 & 110 \\
\hline $\boldsymbol{\alpha}_{50}$ & {$\left[{ }^{\circ}\right]$} & 22,4 & 33,3 & 27,4 & 36,0 \\
\hline $\boldsymbol{\beta}_{80}$ & {$\left[{ }^{0}\right]$} & 11,3 & 10,4 & 13,8 & 30,0 \\
\hline$\gamma$ & {$[0]$} & 7,4 & 4,9 & 3,5 & 0,0 \\
\hline$S M D$ & {$[\mu m]$} & 70,0 & 68,1 & 55,9 & 49,6 \\
\hline
\end{tabular}

Tabelle 4.1.: Kenndaten der ausgewählten Spray-Varianten bei $p_{K r}=3.0 \mathrm{bar}$

der jeweiligen Spraywinkel sind die vier Spray-Targeting-Varianten in Abbildung 4.24 grafisch dargestellt. Bei näherungsweise konstantem statischen Durchfluss $Q_{\text {stat }}$ wird mit Injektor D eine Variation des $\alpha_{50^{-}}$Winkels, mit Injektor E eine Variation des $\beta_{80^{-}}$ Winkels und mit Injektor $\mathrm{G}$ eine Variante mit besonders großem $\beta_{80^{-}}$Winkel und folglich mit besonders kleinem SMD untersucht. Allgemein gilt, dass der $\beta_{80}$-Winkel eines Sprays den mittleren Tropfendurchmesser SMD direkt beeinflusst. Je größer der $\beta_{80^{-}}$ Winkel, desto besser gelingt die Aufbereitung des Sprays, kleine Sauterdurchmesser sind die Folge. Der Neigungswinkel des Sprays $\gamma$ gegenüber der Injektorachse wird nicht gesondert variiert, sondern gegebenenfalls für eine Feinausrichtung des Sprays innerhalb des Einlasskanals justiert.

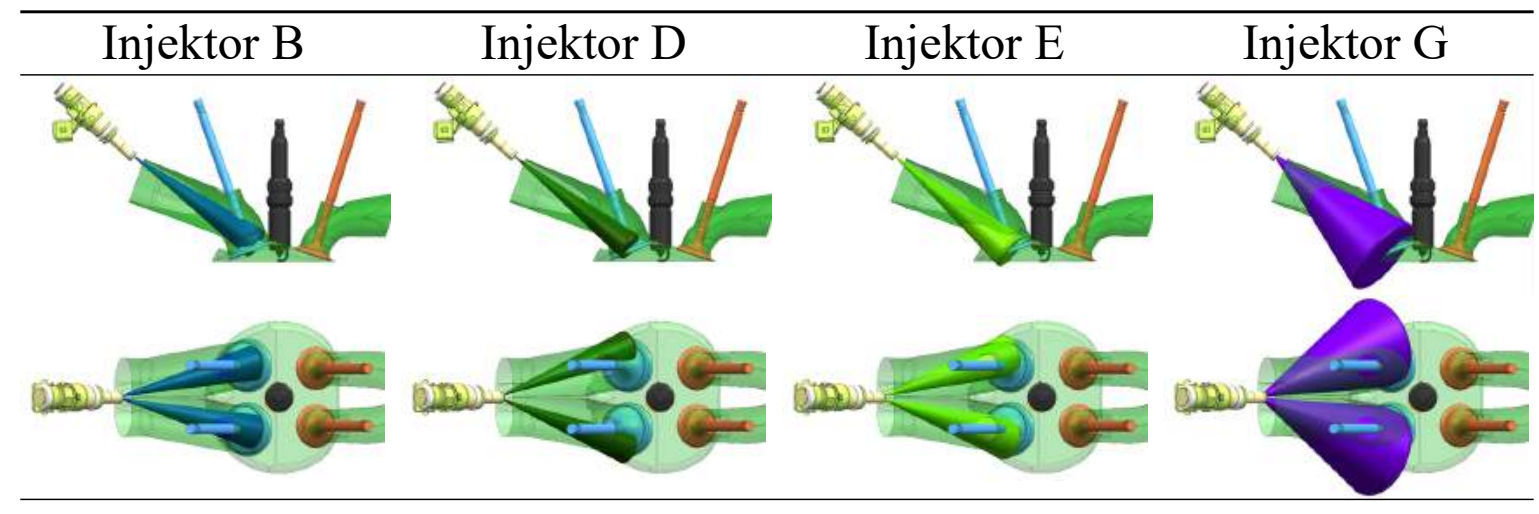

Abbildung 4.24.: CAD-Visualisierungen der für diese Studie ausgewählten SprayAuslegungen in der Seitenansicht (oben) und Draufsicht (unten)

Die Versuchsdurchführung in Form von Lastsprüngen erfolgt erneut mit der in Abbildung 4.15 und Abbildung 4.16 vorgestellten Methode, da sowohl bei warmem als auch bei kaltem Motor mit HSVE gemessen wird. Die im Folgenden vorgestellten Ergebnisse beschränken sich jedoch auf Messungen mit Lastsprüngen auf die höchste Ziellast von $I M E P_{2}=12$ bar. Zum einen ist bei hoher Motorlast der Optimierungsbedarf bezüglich PN-Emissionen besonders groß, wie Kapitel 4.2.1 zeigt, zum anderen sind hierbei die Unterschiede der untersuchten Spray-Varianten besonders deutlich. 
Die Mittelwerte der PN-Konzentration aus jeweils drei aufeinanderfolgenden Lastsprüngen bei warmem Motor sind in Abbildung 4.25 für vier verschiedene Injektoren dargestellt. Ganz links sind die Auswertungen der Parameterkombination vorgelagerte Einspritzung (cVI) und deaktivierte Ladungsbewegungsklappe (LBK =0) zu sehen. Hier ist der besonders große Einfluss des Spray Targetings hervorzuheben. Zwischen

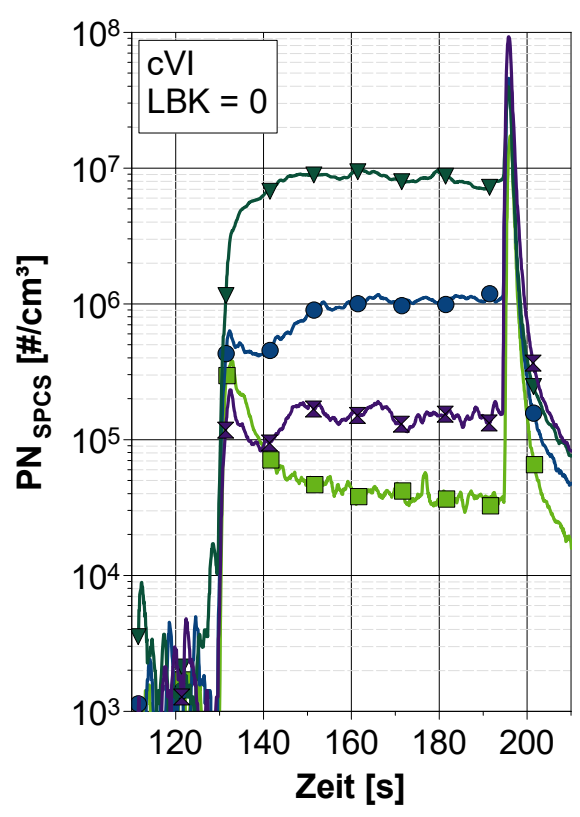

Abbildung 4.25.: PN-Emissionen

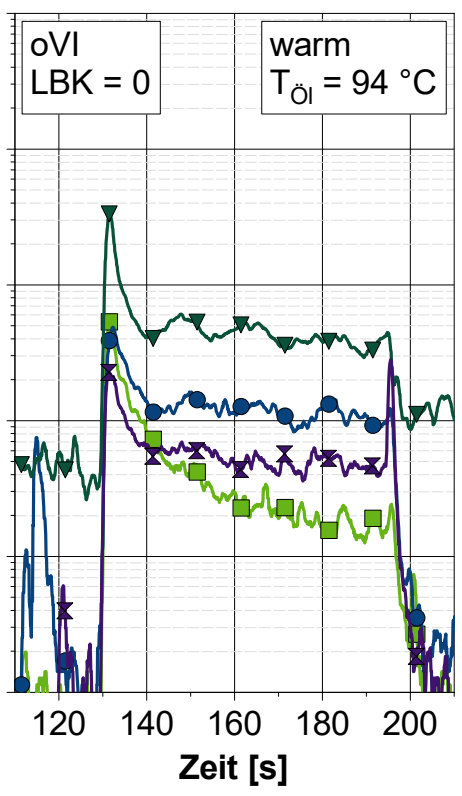
$T_{\ddot{\mathrm{O}} l}=94{ }^{\circ} \mathrm{C}$ für drei Parameter-Kombinationen

der PN-Konzentration des hier sehr guten Injektors E und der des Injektors D liegt mehr als Faktor 100. Eine Vergrößerung des $\alpha_{50}$-Winkels bei ähnlichem $\beta_{80}$-Winkel, wie bei Injektor D, ist somit zu vermeiden. Große $\alpha_{50}$-Winkel sind in Kombination mit großen $\beta_{80}$-Winkel hingegen unschädlich, wie anhand von Injektor E und G ersichtlich wird. Dieselben beiden Injektoren zeigen weiterhin, dass es sich im Sinne einer PNOptimierung lohnt, stets große $\beta_{80}$-Winkel anzustreben. Für eine gute Gemischaufbereitung muss dabei zwangsläufig auch der $\alpha_{50}$-Winkel entsprechend vergrößert werden, um eine zu starke Überlappung der linken und rechten Spray-Keulen zu vermeiden.

Die Kombination aus saugsynchroner Einspritzung (oVI) und LBK $=0$ befindet sich in der Mitte von Abbildung 4.25. Von dieser Parameterkombination profitieren die Sprays von Injektor B und D. Die im ersten Fall bereits gut abschneidenden Injektoren E und $\mathrm{G}$ erreichen hierdurch nur eine geringe bis gar keine Verbesserung.

Rechts im Bild erfolgen die Auswertungen für die Kombination aus saugsynchroner Einspritzung (oVI) und angestellter Ladungsbewegungsklappe (LBK = 1). Diese Kombination führt zu einer messbaren Reduktion der PN-Emissionen für alle Sprays. Demnach zeigt sich erneut das Potenzial einer aktivierten Ladungsbewegungsklappe, 
welches im vorangegangen Teilkapitel 4.2.5 ausführlich beschrieben ist. Besonders interessant ist, dass die Unterschiede zwischen den Sprays hierbei nahezu verschwinden. Der Einfluss der Ladungsbewegungsklappe ist dominierend im Hinblick auf die Gemischbildung, da die infolge von Sekundärzerstäubung zerkleinerten Spraytropfen der Luftströmung besser folgen können. Es kommt zu einer Umlenkung der ursprünglichen Spraystrahlen. Dadurch kann nahezu unabhängig vom originalen Spraytargeting die Wandfilmbildung im Brennraum reduziert werden, was eine messbar geringere PNEmission ermöglicht.

Die in analoger Weise ausgewerteten Messdaten der Lastsprünge mit kaltem Motor sind in Abbildung A.18 im Anhang dargestellt. Zunächst sei darauf hingewiesen, dass im Vergleich zum warmen Motor deutlich höhere PN-Konzentrationen für alle SprayVarianten verzeichnet werden. Alle Messwerte rangieren nach Erreichen der Ziellast oberhalb von $10^{7} \# / \mathrm{cm}^{3}$, weswegen für die PN-Konzentration von einer logarithmischen zu einer linearen Achsenskalierung übergegangen wird. Weiterhin ist festzuhalten, dass die Unterschiede zwischen den Spray-Varianten geringer ausfallen als bei warmem Motor. Zwischen einem günstigen und ungünstigen Spray-Targeting liegt nun maximal Faktor zwei, anstelle von Faktor 100 bei warmem Motor.

Bei einer Kombination aus vorgelagerter Einspritzung (cVI) und deaktivierter Ladungsbewegungsklappe (LBK $=0)$ sind zwei Gruppierungen zu erkennen, welche sich im Wesentlichen durch ihren $\beta_{80}$-Winkel und folglich auch SMD unterscheiden. Die Injektoren $\mathrm{B}$ und $\mathrm{D}$ mit kleinen $\beta_{80}$-Winkeln und verhältnismäßig hohen PN-Emissionen, sowie die Injektoren E und $G$, welche durch ihre bessere Sprayaufbereitung in etwa halb so hohe PN-Konzentrationen ermöglichen. Eine saugsynchrone Einspritzung ohne verstärkte Ladungsbewegung führt nur für Injektor B zu einer messbaren Verbesserung. Für alle anderen Spray-Varianten ergeben sich sehr ähnliche Resultate. Wie auch bei warmem Motor nimmt mit Anstellung der Ladungsbewegungsklappe (LBK = 1) bei saugsynchroner Einspritzung der Einfluss des Spray-Targetings ab. Mit allen Injektoren kann damit aber erneut eine deutliche PN-Reduktion erzielt werden.

Es gilt nun den genauen Ursachen für die besonders hohen Partikelemissionen mit Injektor D und B, sowie für die guten Ergebnisse mit Injektor E und G auf den Grund zu gehen. So stellt sich beispielsweise die Frage, welchen Einfluss die untersuchten Spray-Winkel auf die Partikelentstehung im Brennraum haben. Dies soll im Folgenden anhand von HSVE-Analysen untersucht werden. Insbesondere der große Nachteil hinsichtlich der PN-Konzentration mit Injektor D bei vorgelagerter Einspritzung und deaktivierter Ladungsbewegungsklappe bei warmem Motor sei in diesem Zusammenhang in Abbildung 4.26 analysiert.

In der oberen Bildhälfte ist eine beispielhafte Verbrennung sowie die zeitgleich, da vorgelagert, stattfindende Einspritzung zu sehen. In der unteren Bildhälfte ist derselbe Betriebspunkt mit Injektor G visuell erfasst. Bei genauer Betrachtung der Sprays im Einlasskanal, sind die Unterschiede im Bezug auf die $\alpha_{50^{-}}$Winkel gut erkennbar, wie die schwarze, durchgezogene Linie gegenüber der Strich-Punkt-Linie verdeutlicht. Obwohl ein vorgelagertes Einspritztiming vorliegt, ist bei angepasstem Spray-Targeting 


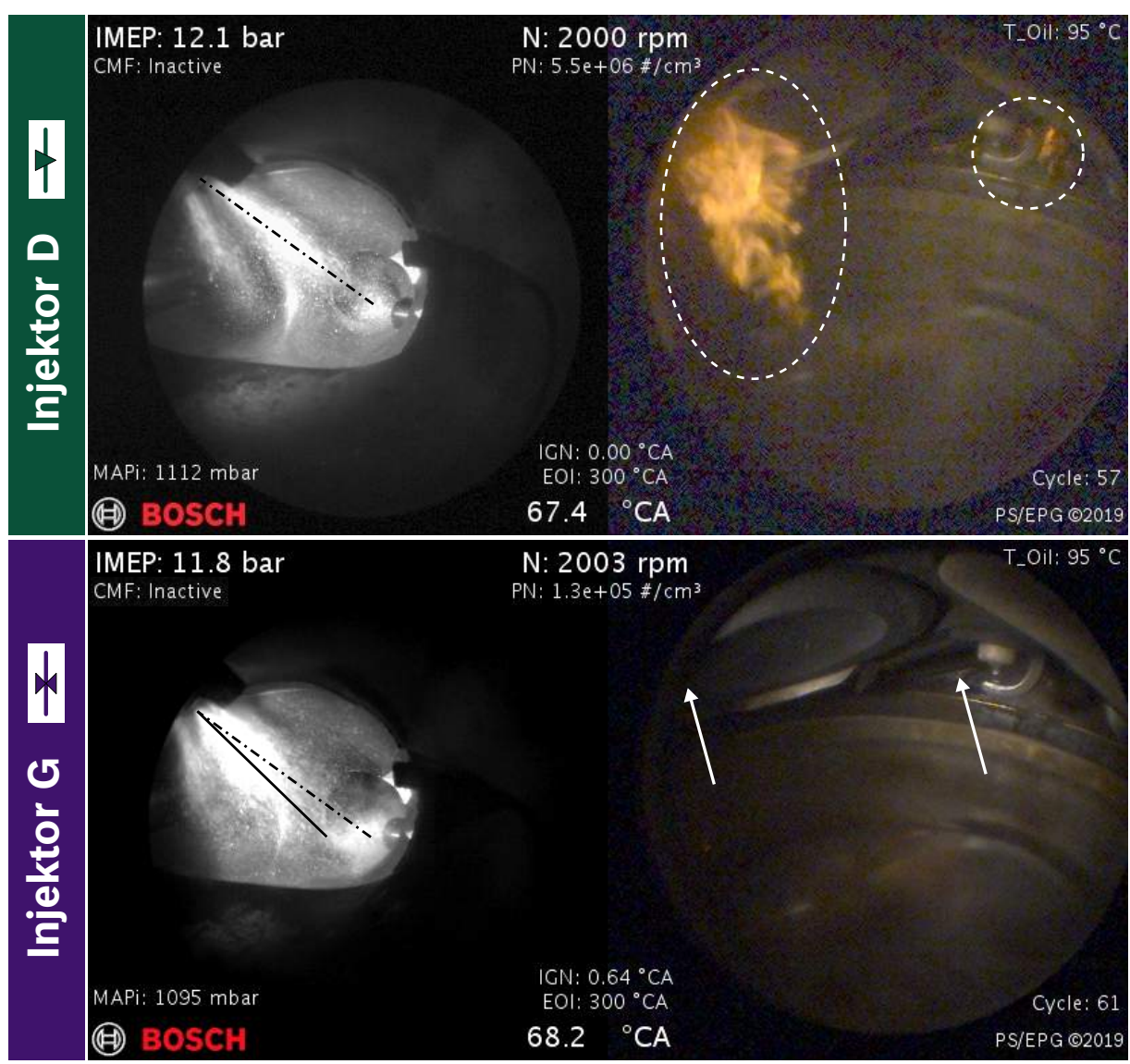

Abbildung 4.26.: Gegenüberstellung von Spray-Targeting und Verbrennung von Injektor $D$ und $G$ mittels HSVE hinsichtlich Partikelentstehung bei $I M E P_{2}=12$ bar, $\mathrm{cVI}, \mathrm{LBK}=0$ und warmem Motor

von Injektor G keine Partikelentstehung mehr an den Einlassventilsitzen erkennbar (weiße Pfeile). Das Targeting von Injektor D führt hingegen zu ausgeprägten Diffusionsflammen an den äußeren Rändern der Einlassventile (gestrichelte Ellipsen). Eine statistische Auswertung der beschriebenen Messungen ist in Abbildung A.19 im Anhang zu finden. Inwiefern diese offenbar ungünstige Kombination der Spraywinkel von Injektor D zur Bildung von Kraftstoffwandfilmen führt, wird im Folgenden mit Hilfe von Wandfilm-Analysen am Transparentmotor genauer untersucht.

In Abbildung 4.27 werden die räumlichen Auftretenswahrscheinlichkeiten von Kraftstoffwandfilmen für Injektor D und $G$ bei vorgelagerter Einspritzung und LBK $=0$ gegenübergestellt. Perspektive „1-V" zeigt eine deutliche Akkumulation von Kraftstoff am äußeren Rand der Einlassventile mit Injektor D. In Perspektive „1-L“ und „2-L“ sind statistisch weniger relevante, vereinzelte Tropfen auf der Zylinderlaufbahn sichtbar, welche hinsichtlich PN eine untergeordnete Rolle spielen. Bei den Messungen am Transparentmotor mit Injektor $\mathrm{G}$ hingegen können bei dieser Parameterkombination keinerlei Wandfilme detektiert werden. Dies ist einerseits auf die Auswertung von lediglich zehn Zyklen zurückzuführen. Bei Betrachtung von 30 anstelle von zehn 

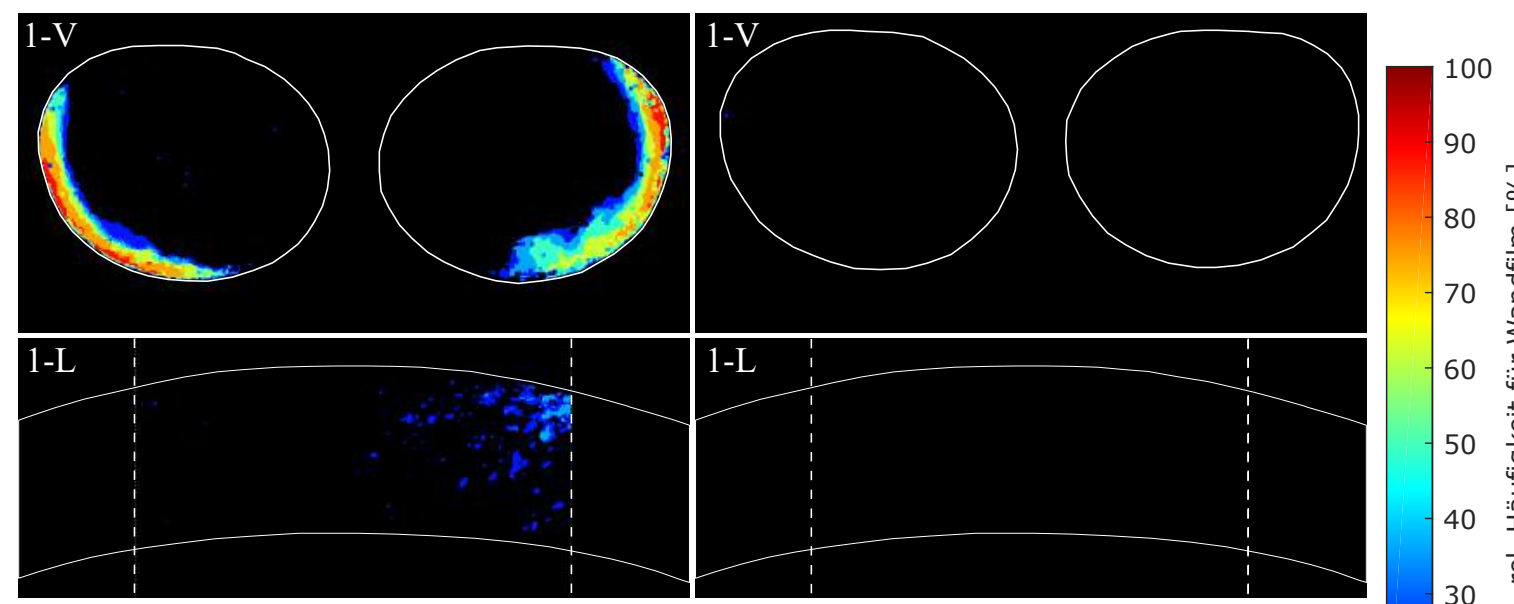

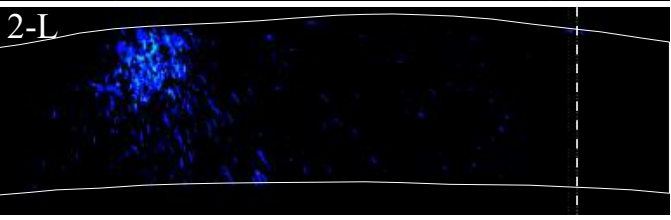

Injektor D | cVI | LBK0

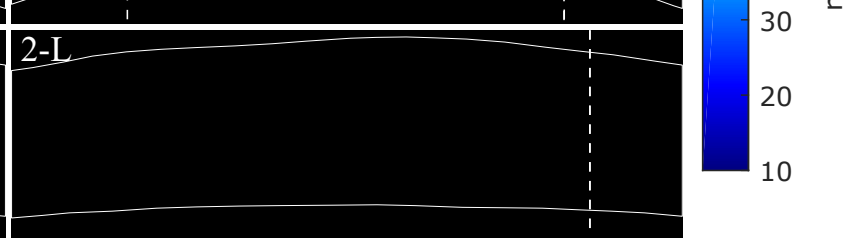

Injektor $\mathrm{G}$ | cVI | LBK0

Abbildung 4.27.: Gegenüberstellung der Auftretenswahrscheinlichkeit von Wandfilm nach 10 Zyklen bei vorgelagerter Einspritzung (cVI) und LBK $=0$ mit Injektor D (links) und Injektor G (rechts)

Zyklen sind auch bei Injektor G Wandfilme detektierbar, was in Abbildung A.20 im Anhang eingesehen werden kann. Ein deutlicher Vorteil von Injektor G ggü. Injektor D ist jedoch auch bei dieser Auswertung klar ersichtlich. Andererseits spricht die Abwesenheit von regulär messbaren Wandfilmen mit Injektor $\mathrm{G}$ für dünne, homogen verteilte Wandfilme auf den Einlassventilen auf der Einlasskanal-Seite, welche keinen relevanten Kraftstofftransport in den Brennraum in flüssiger Form zur Folge haben.

Zur Erarbeitung der erforderlichen Kriterien für eine PN-optimale Spray-Auslegung wird im Folgenden ein weiterer Vergleich zweier Injektoren mit Hilfe von optischen Messmethoden angestellt. Der Fokus liegt hierbei auf saugsynchronen Einspritztimings. Dazu erfolgt in Abbildung 4.28 zunächst eine statistische Gegenüberstellung der im Brennraum erfassten Diffusionsflammen von Injektor B und Injektor G bei kaltem Motor.

Bei beiden Injektoren sind trotz der saugsynchronen Einspritzung Diffusionsflammen an den Einlassventilsitzen zu verzeichnen. Besonders auffällig ist die konzentrierte Partikelquelle an der Zylinderlaufbahn unterhalb der Auslassventile mit Injektor B. Hierbei gilt es noch zu bedenken, dass der tatsächliche Ursprungsort der Diffusionsflammen auch rechts unterhalb des endoskopischen Sichtfelds liegen kann, sodass eine subjektive Unterschätzung nicht ausgeschlossen werden kann. Mit Injektor G sind demgegenüber drei statt zwei Quellen für Diffusionsflammen zu erkennen. Auch seitlich an der Zylinderlaufbahn, also zwischen Einlass- und Auslassventil, werden Diffusionsflammen erfasst. Jedoch sind diese Partikelquellen weniger lokal konzentriert. 


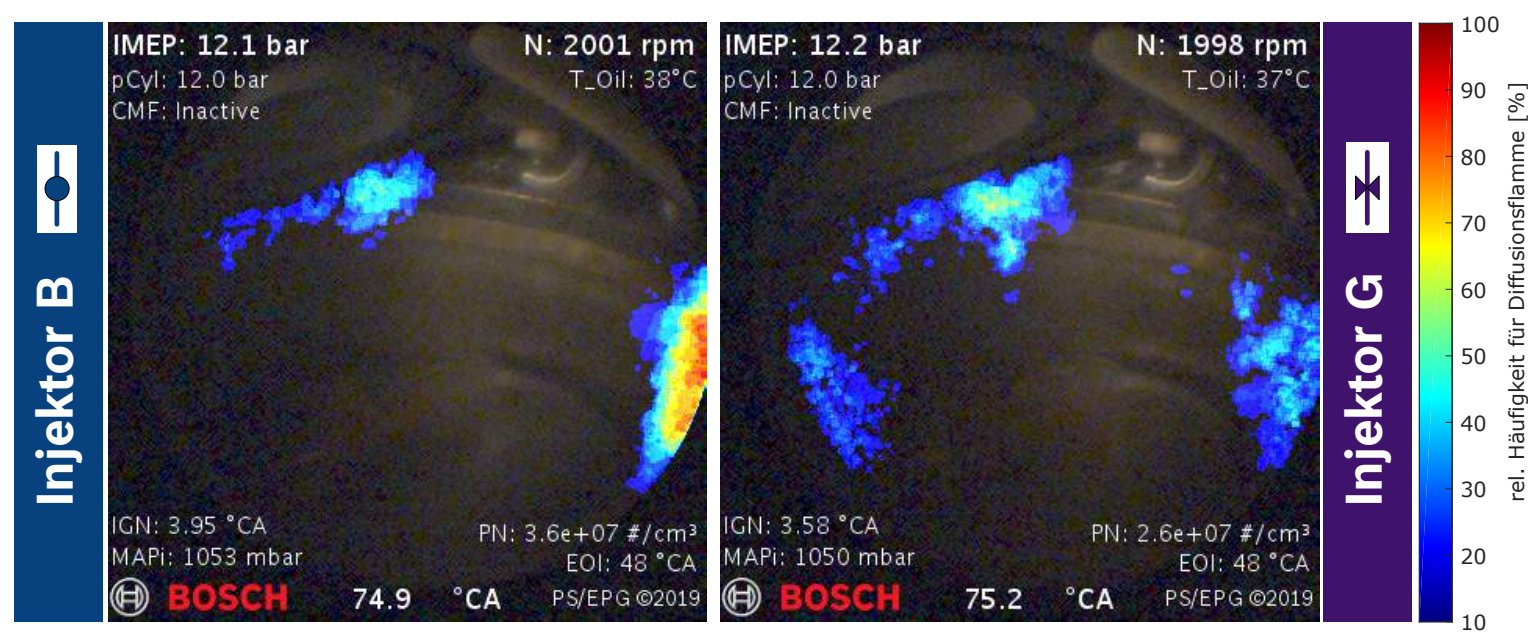

Abbildung 4.28.: Gegenüberstellung der räumlichen Auftretenswahrscheinlichkeit von Diffusionsflammen mit Injektor $\mathrm{B}$ und $\mathrm{G}$ bei $I M E P_{2}=12 \mathrm{bar}$, oVI, $\mathrm{LBK}=0$ und kaltem Motor

Besonders die Flammen an der Zylinderlaufbahn sind auch statistisch weniger häufig dokumentiert, wie die bläulichen Einfärbungen erkennen lassen. Es liegen somit Indizien vor, dass eine möglichst homogene Verteilung des Kraftstoffes und somit mehrere kleinere Wandfilme gegenüber einer starken Akkumulation von Kraftstoff in wenigen oder einem einzelnem Wandfilm zu bevorzugen ist. Ob dieser Eindruck hinsichtlich der Wandfilme bestätigt werden kann, soll eine Analyse anhand von TransparentmotorMessungen in Abbildung 4.29 zeigen.

Die verhältnismäßig kleinen Spray-Winkel von Injektor $B$ mit $\alpha_{50}=22,4^{\circ}$ und $\beta_{80}=11,3^{\circ}$ führen insgesamt zu sehr kompakten Spraykeulen. Dies hat bei saugsynchroner Einspritzung einen sehr ausgeprägten Wandfilm an der Zylinderlaufbahn unterhalb der Auslassventile zur Folge. Nahezu der gesamte Sichtbereich in Perspekive "1-L" wird davon eingenommen. Außerdem kann durch die konzentrierte, kleine Auftrefffläche an der Ober- und Innenseite der Einlassventile auch dort eine Wandfilmbildung nicht vermieden werden.

Kraftstoffwandfilme können zwar auch mit Injektor $\mathrm{G}$ nicht vollständig vermieden werden, aber die sehr großen Spray-Winkel, mit $\alpha_{50}=36,0^{\circ}$ und $\beta_{80}=30,0^{\circ}$, tragen dazu bei, Akkumulation an gewissen Orten im Brennraum zu vermeiden. Folglich entstehen auf mehrere Stellen verteilte Wandfilme, wie in Perspektive „1-L" und „2-L" zu sehen ist. Der, dank des großen $\beta_{80}$-Winkels, klein ausfallende SMD von Injektor G trägt zusätzlich zu einer verbesserten Gemischbildung bei. So kann eine Reduzierung der gesamten Wandfilmfläche aller drei Perspektiven von Injektor G mit $244 \mathrm{~mm}^{2}$ auf etwa $32 \%$ im Vergleich zur Wandfilmfläche von Injektor B $\left(744,5 \mathrm{~mm}^{2}\right)$ erreicht werden. An dieser Stelle sei noch auf die nahezu vollständig rote Einfärbung des rechten Wandfilms von Injektor G in Perspektive „2-L" eingegangen, welche zum Ausdruck bringt, dass eine Benetzung an dieser Stelle sehr häufig, also sehr wahrscheinlich ist. 
Dies darf nicht mit einer vermeintlichen Wandfilmmasse gleichgesetzt werden, da die

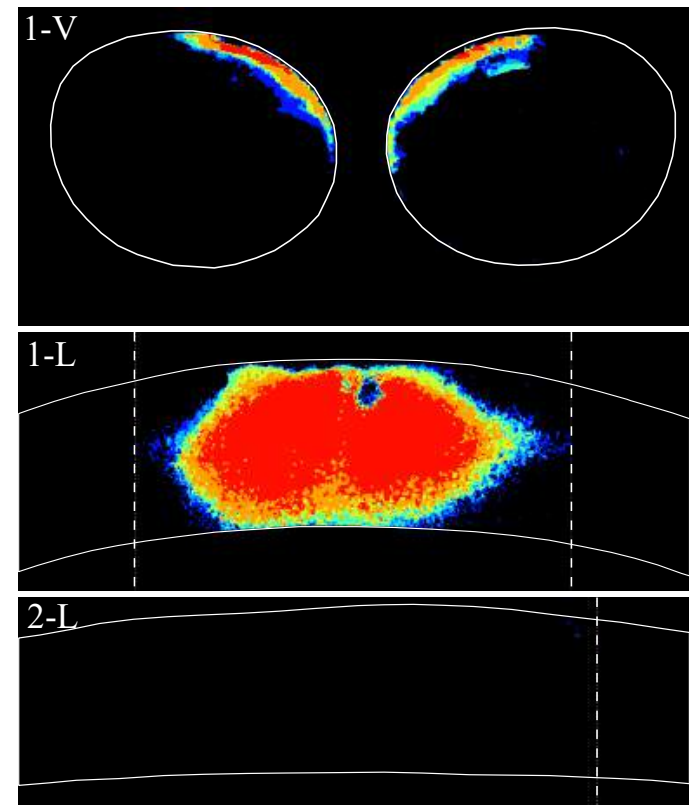

Injektor B $\mid$ oVI $\mid \mathrm{LBK}=0$

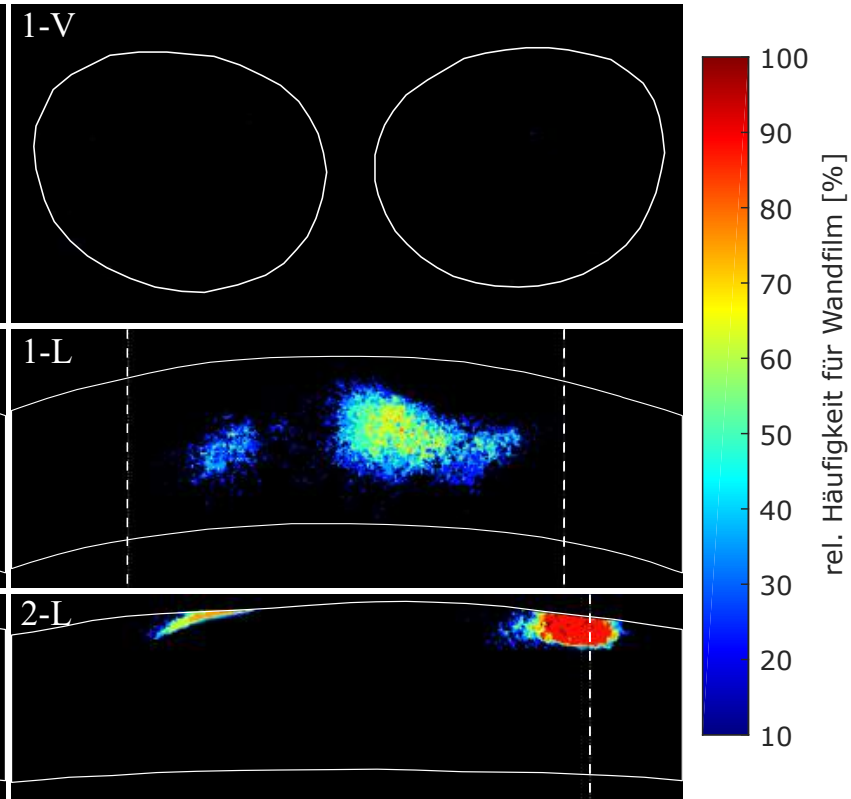

Injektor G $\mid$ oVI $\mid \mathrm{LBK}=0$

Abbildung 4.29.: Gegenüberstellung der Auftretenswahrscheinlichkeit von Wandfilm nach 10 Zyklen bei saugsynchroner Einspritzung (oVI) und LBK $=0$ mit Injektor B (links) und Injektor G (rechts)

Wandfilmdicke nicht bekannt ist. Es kann sich also theoretisch um eine dünne und häufig vorkommende Benetzung handeln.

\section{Zwischenfazit}

An dieser Stelle seien die anhand der vorgestellten Spray-Targeting Studie gewonnenen Erkenntnisse kurz zusammengefasst. Erste Kriterien für eine PN-optimale SprayAuslegung bei Single-Konfiguration (ein Injektor pro Zylinder) können festgehalten werden:

- Große $\beta_{80}$-Winkel sind vorteilhaft.

- Große $\alpha_{50}$-Winkel sind nur in Kombination mit großen $\beta_{80^{-}}$Winkeln zielführend.

- Insbesondere die Kombination kleine $\beta_{80}$-Winkel und große $\alpha_{50}$-Winkel ist zu vermeiden.

Die guten Resultate der Injektoren mit großen $\beta_{80^{-}}$Winkeln im Hinblick auf PNEmissionen können jedoch nicht eindeutig dem jeweiligen Spray-Targeting zugeschrieben werden. Möglicherweise ist der bei diesen Injektoren verhältnismäßig kleine mittlere Tröpfchendurchmesser (SMD) ausschlaggebend. Um die beiden Einflussparameter Spray-Targeting und Tröpfchengröße unabhängig voneinander bewerten zu können, wird die, im folgenden Teilkapitel vorgestellte, Twin-Targeting Studie durchgeführt. 


\subsubsection{Spraywinkel bei Twin-Konfiguration}

Durch die Verwendung eines modifizierten Saugrohrs, sowie durch die Anfertigung eines entsprechenden Kraftstoff-Rails für den hydraulischen Anschluss von acht, anstelle von vier, Injektoren ist es möglich denselben Vollmotor für eine Twin-Targeting Studie zu ertüchtigen. Somit steht für jedes Einlassventil ein Injektor zur Verfügung. Jedem Zylinder sind folglich zwei Injektoren zugeordnet. In Abbildung 4.30 sind die für diese Studie angefertigten Spray-Auslegungen J, K und L tabellarisch dargestellt.

\begin{tabular}{|c|c|c|c|c|}
\hline Injektor & $\mathbf{J}$ & $\mathbf{K}$ & $\mathbf{L}$ & $\mathbf{E}$ \\
\hline Konfiguration & Twin & Twin & Twin & Single \\
\hline Düsentyp & $\mathrm{C}$ & $\mathrm{C}$ & $\mathrm{C}$ & E \\
\hline$\alpha / \beta\left[^{\circ}\right]$ & $12,7 /-$ & $20,0 /-$ & $20.2 /-$ & $27,4 / 13,8$ \\
\hline$\gamma \quad\left[{ }^{\circ}\right]$ & 7,2 & 12,0 & 8,7 & 3,5 \\
\hline$Q_{\text {stat }}[\mathrm{g} / \mathrm{min}]$ & 75 & 75 & 75 & 106 \\
\hline SMD $[\mu \mathrm{m}]$ & 61,2 & 59,3 & 53,5 & 55,9 \\
\hline \multicolumn{5}{|l|}{ Seitenansicht } \\
\hline Draufsicht & & & & \\
\hline
\end{tabular}

Abbildung 4.30.: Kenndaten und CAD-Visualisierungen der Spray-Auslegungen der TwinSRE-Injektoren bei Rotation um $0^{\circ}$ („oben“) sowie der Single-Vergleichsbasis Injektor E

Diese Sprays bilden lediglich eine Spray-Keule aus. Bei Injektoren der Marke Bosch EV14 handelt es sich hierbei um Düsen vom „Typ C“. Bei Injektoren für SingleKonfigurationen mit zwei Spray-Keulen kommen Düsen vom „Typ E“ zum Einsatz, wie es auch bei der Single-Vergleichsbasis, Injektor E, der Fall ist. Außerdem sei erwähnt, dass es bei Injektoren vom Typ C keinen $\beta_{80}$-Winkel gibt. Der Öffnungswinkel der Spraykeule wird hierbei durch einen $\alpha_{80}$-Winkel beschrieben (siehe Abbildung 2.2b. Der Öffnungswinkel wird in zwei Stufen variiert, $\alpha_{80}=13^{\circ}$ bei Injektor $\mathrm{J}$ und $\alpha_{80}=20^{\circ}$ für Injektor $\mathrm{K}$ und L. Alle Twin-Injektoren werden mit einem $\gamma$ Winkel ausgelegt. Dieser ist bei Injektor J und L groß, bei Injektor K bewusst sehr groß gewählt. Da sich neben dem Öffnungswinkel auch der Verkippungswinkel $\gamma$ auf die Zerstäubungsqualität und somit auf den Tropfendurchmesser auswirkt, ergeben sich verschiedene SMD-Werte für die drei Twin-Auslegungen.

Aufgrund der verwendeten $\gamma$-Winkel eröffnet sich nun die Möglichkeit, das SprayTargeting durch symmetrische Verdrehung der C-Typ-Injektoren in ihrer Einbaulage zu verändern. Diese Verdrehung ist in Abbildung 4.31 am Beispiel von Injektor J grafisch dargestellt. Die Auswirkungen durch das mittels Rotation veränderte Spray- 


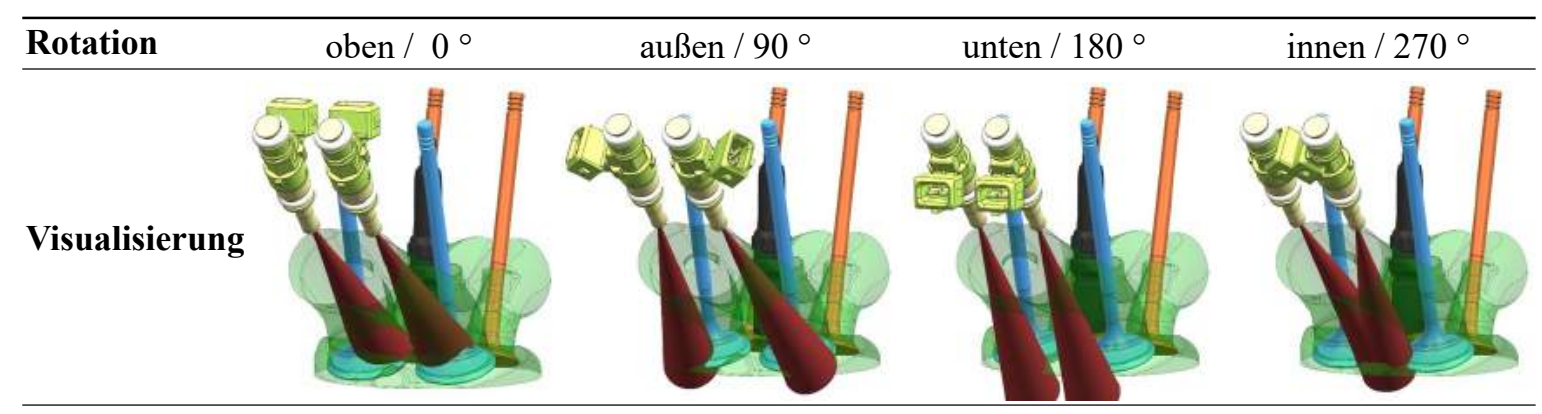

Abbildung 4.31.: Variation des Spray-Targetings durch Rotation der Einbaulage am Beispiel von Injektor J

Targeting auf die Partikelemissionen werden an den vier Positionen „oben“, „außen“, „unten“ und „innen“ ermittelt. Im Folgenden werden diese Positionen auch durch Rotationswinkel im Uhrzeigersinn bezüglich des rechten Einlasskanals gekennzeichnet $\left(0^{\circ}, 90^{\circ}, 180^{\circ}\right.$ und $\left.270^{\circ}\right)$. Somit ist es möglich den Einfluss eines veränderten SprayTargtings mit unverändertem Tropfengrößenspektrum hinsichtlich PN-Emissionen zu bewerten. Durch die Vermessung der drei beschriebenen Injektormuster J, K und L mit ihrem jeweiligen SMD, kann der Parameter Tropfengröße in derselben Studie parallel zum Targeting-Einfluss betrachtet werden. Ziel dieser Studie ist es ausdrücklich nicht, das Potenzial einer Twin-Konfiguration zur Reduktion der Partikelemissionen gegenüber einer Single Konfiguration zu bewerten. Vielmehr geht es um die getrennte Bewertung der Einflussparameter Spray-Targeting und mittlerer Sauterdurchmesser SMD.

Die in dieser Twin-Targeting-Studie variierten Parameter decken sich im wesentlichen mit denen der im vorangegangen Teilkapitel vorgestellten Untersuchung. Zu den somit bereits bekannten Variationsparametern Motorlast, Motortemperatur, Einspritztiming, Stellung der Ladungsbewegungsklappe und Spray-Layout kommt nun noch die Rotation der Injektoren hinzu. Die jeweilige Motorlast wird außerdem stationär eingeregelt. Lastsprünge auf die gewünschte Ziellast werden nur bei optischen Messungen mit HSVE durchgeführt. Bei den im Folgenden dargestellten Ergebnissen handelt es sich um Mittelwerte aus drei Wiederholungen.

Die Ergebnisse hinsichtlich der Partikelkonzentration in Abhängigkeit der mittels Rotation veränderten Spray-Targetings ist für alle drei Spray-Auslegungen in Abbildung 4.32 für warmen Motor $\left(T_{\ddot{\mathrm{O}} l}=93^{\circ} \mathrm{C}\right)$, IMEP $=12$ bar und LBK $=0$ dargestellt. Auf der radialen Achse des Polarkoordinatensystems sind die gemittelten PN-Konzentrationen in logarithmischer Skalierung aufgetragen. Die Winkelkoordinate stellt die Rotation der Injektoreinbaulage dar. Auf der linken Bildhälfte sind Messungen mit vorgelagertem Einspritztiming (cVI) ausgewertet. Hierbei dominiert der Einfluss der Sprayqualität, welche wiederum durch die Spraywinkel $\alpha_{80}$ und $\gamma$, sowie durch den SMD geprägt ist. Injektor L erzielt mit großem $\alpha_{80}$-Winkel, geringem $\gamma$-Winkel und kleinstem SMD $=53,5 \mu m$ die geringsten Partikelkonzentrationen. Die einzige Ausnahme stellt die Rotation nach unten dar, bei der Injektor J minimal 


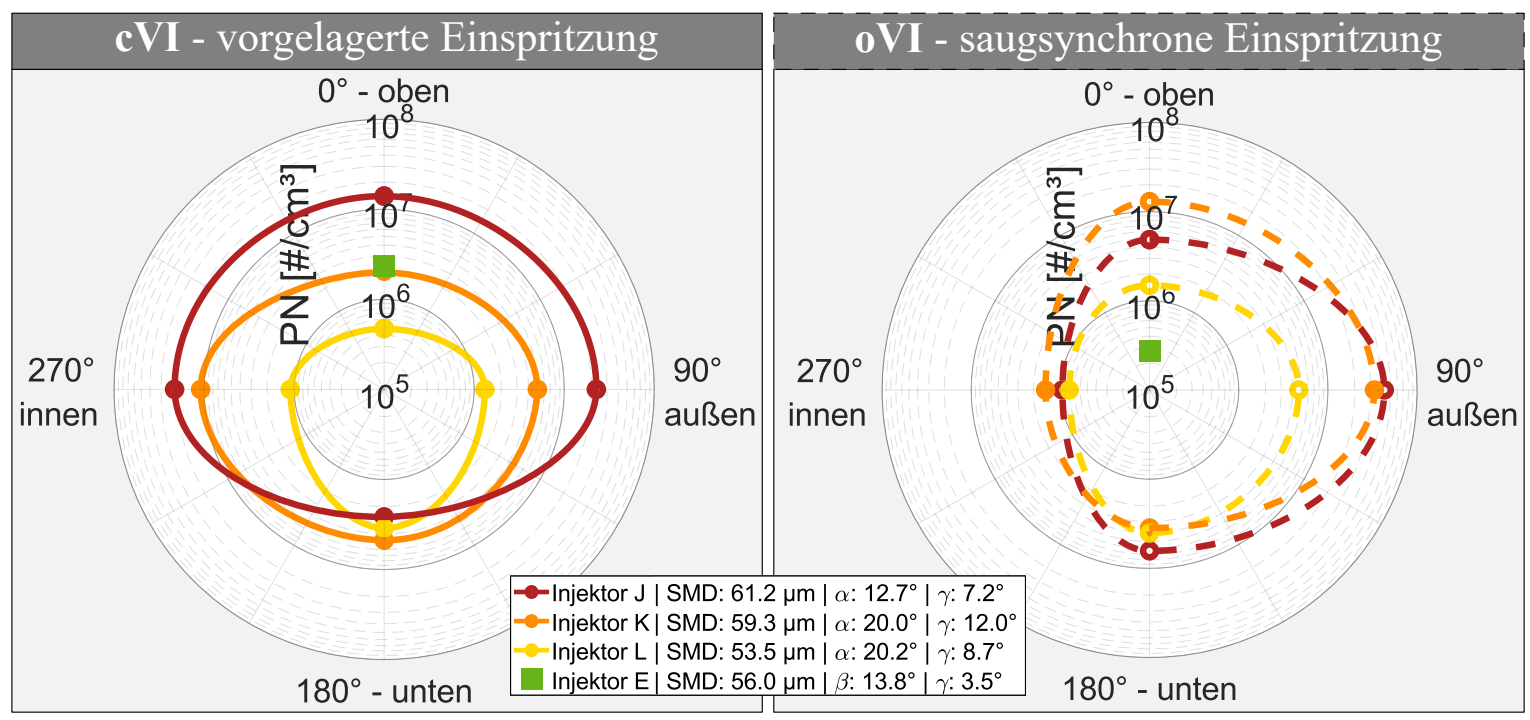

Abbildung 4.32.: PN-Emissionen in Abhängigkeit des Spray-Targetings, variiert durch Rotation der Injektoren, bei IMEP $=12$ bar, $\mathrm{LBK}=0$ und warmem Motor

besser abschneidet. Es besteht bei vorgelagerter Einspritzung jedenfalls eine sehr geringe Abhängigkeit vom durch die Injektor-Rotation veränderten Targeting. Bei den Messungen mit saugsynchronem Einspritztiming ist hingegen ein sehr deutlicher Einfluss des Spray-Targetings durch Rotation der Injektoreinbaulage erkennbar. So wird beispielsweise deutlich, dass eine Orientierung des Sprays nach außen $\left(90^{\circ}\right)$ besonders schädlich wirkt im Hinblick auf die Partikelemission. Eine Verdrehung der Sprays nach innen $\left(270^{\circ}\right)$ ist indes für alle drei Injektoren vorteilhaft. Der positive Einfluss von kleinen Tropfengrößen (SMD) und günstigen Spraywinkeln ist zwar prinzipiell erkennbar, ist jedoch bei diesen Messungen mit saugsynchroner Einspritzung von untergeordneter Bedeutung. Der Einfluss des Spray-Targetings ist hierbei größer einzustufen, da sich die PN-Konzentration mit demselben Injektor und somit konstantem Tropfengrößenspektrum je nach Einbaulage um mehr als Faktor zehn verändert.

Die Auswertungen der Messungen bei kaltem Motor $\left(T_{\ddot{\mathrm{O}} l}=35^{\circ} \mathrm{C}\right)$ sind in ähnlicher Darstellungsweise in Abbildung A.21 im Anhang veranschaulicht. Aufgrund des deutlich höheren PN-Niveaus kommt hier jedoch eine linear skalierte, radiale Achse für die Partikelkonzentration zum Einsatz. Die bei warmem Motor identifizierten Effekte kommen bei kaltem Motor noch deutlicher zur Geltung. Bei vorgelagerter Einspritzung (cVI) ist erneut die Aufbereitungsqualität der Sprays ausschlaggebend. Nahezu unabhängig von der Injektoreinbaulage, und somit unabhängig vom Spray-Targeting, sind Injektoren mit größerem $\alpha_{80}$-Winkel, geringem $\gamma$-Winkel und kleinerem SMD im Vorteil hinsichtlich der Partikelemission. Bei saugsynchroner Einspritzung treten auch bei kaltem Motor diese Parameter in den Hintergrund, da hier das durch Injektor-Rotation veränderte globale Targeting den wesentlichen Einflussparameter darstellt.

Den vorangegangenen Ergebnis-Kapiteln ist zu entnehmen, dass Kraftstoffwandfilme an den Einlassventilen und den Einlassventilsitzen maßgeblich für die Partikelemissi- 
onen bei vorgelagerter Einspritzung verantwortlich sind. Nun zeigt sich, dass insbesondere Injektor-Sprays mit kleinen Tröpfchendurchmessern (SMD) diese Wandfilmbildung verringern oder insofern günstig beeinflussen können, als dass es nur noch zu einem verminderten Transport der kanalseitigen Wandfilme an Oberflächen innerhalb des Brennraums kommt, wie zum Beispiel die Unterseite der Einlassventile oder dem Ventilsitz. Bei Injektoren mit kleinem SMD kann möglicherweise ein höherer Anteil der Einspritzmasse in Form von Tropfenverdunstung verflüchtigt werden, wie die Versuche an der Verdunstungskammer nahelegen (siehe Abbildung 4.2 und 4.3). Der bei kleinen Tröpfchen verminderte Impuls kann zudem den Anteil der Tropfen erhöhen, welche in der vor den geschlossenen Einlassventilen ruhenden Luft in der Schwebe verbleiben und nicht an eine Wand gelangen. Auch im Kontext der Spraywinkel-Studie bei Single-Konfiguration ist der Einfluss des SMD bei vorgelagerter Einspritzung sehr deutlich, wie jeweils links in Abbildung 4.25 und A.18 zu erkennen ist. Die mittleren Sauter-Durchmesser dieser Injektoren unterscheiden sich sogar deutlicher als die der, hier diskutierten, Twin-Targeting-Studie.

Dennoch kann noch nicht zweifelsfrei geklärt werden, in welchem Maße bei vorgelagerter Einspritzung (cVI) die Tropfengröße SMD die entscheidende Einflussgröße zur Erreichung der beschriebenen Vorteile hinsichtlich Kraftstoffwandfilme und PNEmission darstellt, da ein Quereinfluss der Spraywinkel $\alpha_{80}$ und $\gamma$ stets gegeben ist. Zwar führen größere Öffnungswinkel $\alpha_{80}$ auch zu geringeren Tropfengrößen, aber ebenso zu größeren Wandfilmflächen. Im Sinne einer raschen Kraftstoffverdunstung sind dünne, großflächig verteilte Wandfilme von Vorteil. Akkumulation von Kraftstoff auf geringer Fläche oder in Spalten wie am Einlassventilsitz sind dahingehend nachteilig und erhöhen das Risiko, dass flüssiger Kraftstoff in den Brennraum transportiert wird. Zudem ist es bemerkenswert, dass bereits kleine Unterschiede hinsichtlich des SMDs, beispielsweise ein Unterschied von 5,8 $\mu \mathrm{m}$ zwischen Injektor K und L, bei vergleichbarem $\alpha_{80}$-Winkel, zu einer erheblichen Reduktion der PN-Emission führen. Im nachfolgenden Kapitel 4.2.8 soll die Sensitivität des Einflussparameters Tropfengröße auf die Partikelemissionen im Rahmen einer Kraftstoffdruckerhöhung daher nochmals isoliert betrachtet werden.

Bei saugsynchroner Einspritzung ist es indessen gut nachvollziehbar, dass das SprayTargeting eine dominierende Rolle im Bezug auf die Entstehung von Kraftstoffwandfilmen im Brennraum spielt, welche Partikelemissionen zur Folge haben. Bei rückblickender Betrachtung der Kapitel 4.2.1 bis 4.2.6 hinsichtlich der Partikelquellen bei saugsynchroner Einspritzung wird deutlich, dass es sich in diesen Fällen schwerpunktmäßig um Wandfilme an der Zylinderlaufbahn handelt. Die Flugbahn der Spray-Tröpfchen ergibt sich nach einer Wechselwirkung zwischen Ansaugströmung und Spray-Targeting. Die Ergebnisse der hier vorgestellten Twin-Targeting-Studie bestätigen nun die Indizien der vorangegangenen Targeting-Studie in Kapitel 4.2.6, dass eine Spray-Ausrichtung nach außen, unabhängig vom Tropfendurchmesser, sehr ungünstig ist. Das erhöhte Risiko für Spraytropfen, welche die Einlassventile an den äußeren Bereichen der Einlasskanäle passieren, die Zylinderlaufbahn bereits nach kurzer Flugbahn im Brennraum zu treffen spielt hierbei sicherlich eine Rolle. Die Tatsache, dass eine Orientierung des 
Kraftstoffsprays nach innen, und somit in Richtung der Brennraummitte, zu bevorzugen ist, stellt eine neue Erkenntnis der Twin-Targeting-Studie dar. Bei einer solchen Spray-Ausrichtung passiert die Mehrzahl der Tropfen die Einlassventile auf der Innenseite des Einlasskanals. Dadurch sind deutlich längere Tropfenflugbahnen innerhalb des Brennraums möglich, wodurch sich die Wahrscheinlichkeit für Tropfenverdunstung vor einem Wandkontakt erhöht.

An dieser Stelle sei ergänzt, dass die teilweise höheren PN-Konzentrationen der TwinInjektoren im Vergleich zur Single-Vergleichsbasis Injektor E nicht auf einen prinzipiellen Nachteil einer Twin-Konfiguration zurückzuführen sind. Vielmehr liegt dies höchstwahrscheinlich an den für diese Studie bewusst groß gewählten $\gamma$-Winkeln, welche sich nachteilig auf die Tropfenaufbereitung auswirken.

\section{Sonderfall Twin-Konfiguration mit aktiver Ladungsbewegungsklappe}

Bei allen bislang diskutierten Daten der Twin-Targeting-Studie handelt es sich um Messungen mit deaktivierter Ladungsbewegungsklappe. Im Folgenden soll auf die Auswirkungen einer aktivierten Ladungsbewegungsklappe in Kombination mit TwinKonfigurationen eingegangen werden. Dies wird aus Gründen der Übersichtlichkeit am Beispiel von Injektor K ausgeführt, welcher einen mittleren SMD aufweist. Zunächst sei daran erinnert, dass eine angestellte Ladungsbewegungsklappe in den Kapiteln 4.2.5 und 4.2.6 insbesondere kombiniert mit saugsynchronem Einspritztiming stets zu einer deutlichen Reduktion der Partikelemissionen führt. In Anbetracht der in Abbildung 4.33 vorgestellten Auswertungen muss dies aber differenzierter bewertet werden.

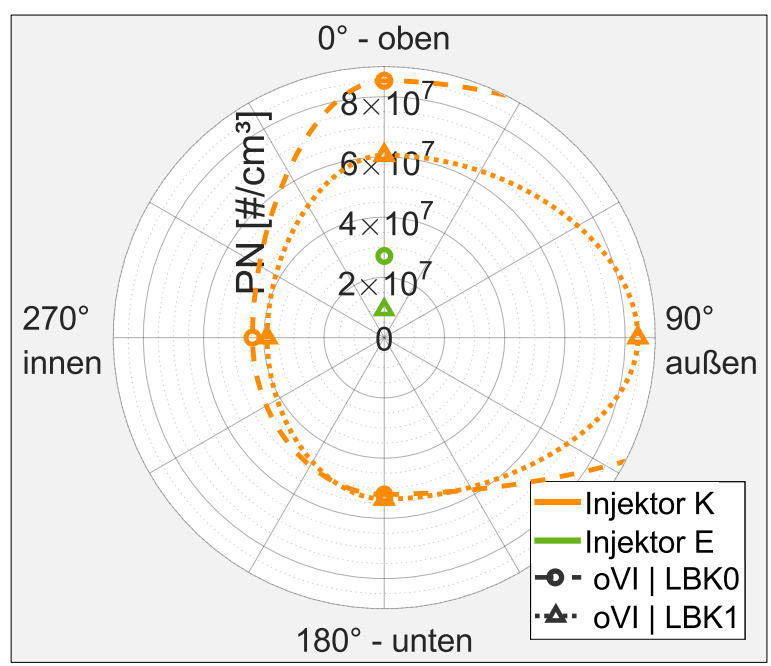

Abbildung 4.33.: Gegenüberstellung der PN-Emissionen mit deaktivierter und aktivierter Ladungsbewegungsklappe in Abhängigkeit des durch Injektor-Rotation veränderten Spray-Targetings, bei IMEP $=12$ bar und kaltem Motor ${ }^{5}$ 
Beim Single-Injektor E führt die Anstellung der Ladungsbewegungsklappe (LBK = 1) bei saugsynchroner Einspritzung zu einer deutlichen Reduktion der Partikelemissionen. Die PN-Konzentration kann mehr als halbiert werden. Im Fall des Twin-Sprays von Injektor $\mathrm{K}$ gelingt lediglich für die ohnehin eher ungünstigen Einbaulagen mit Rotation nach außen $\left(90^{\circ}\right)$ und oben $\left(0^{\circ}\right)$ eine gut messbare Verminderung der PN. Gerade für die, im Hinblick auf geringe Partikelkonzentration, interessante Einbaulage mit Spray-Orientierung nach innen ist die Verbesserung durch Aktivierung der Ladungsbewegungsklappe jedoch marginal. Da die Ursache hierfür zunächst unklar ist, erfolgt eine Wiederholungsmessung unter Zuhilfenahme der High-Speed Video Endoskopie. Eine statistische Auswertung der im Brennraum dokumentierten Diffusionsflammen, sowohl für die Messung mit deaktivierter, als auch mit aktivierter Ladungsbewegungsklappe, zeigt Abbildung 4.34. Die Auswirkungen des ursprünglichen Targetings mit
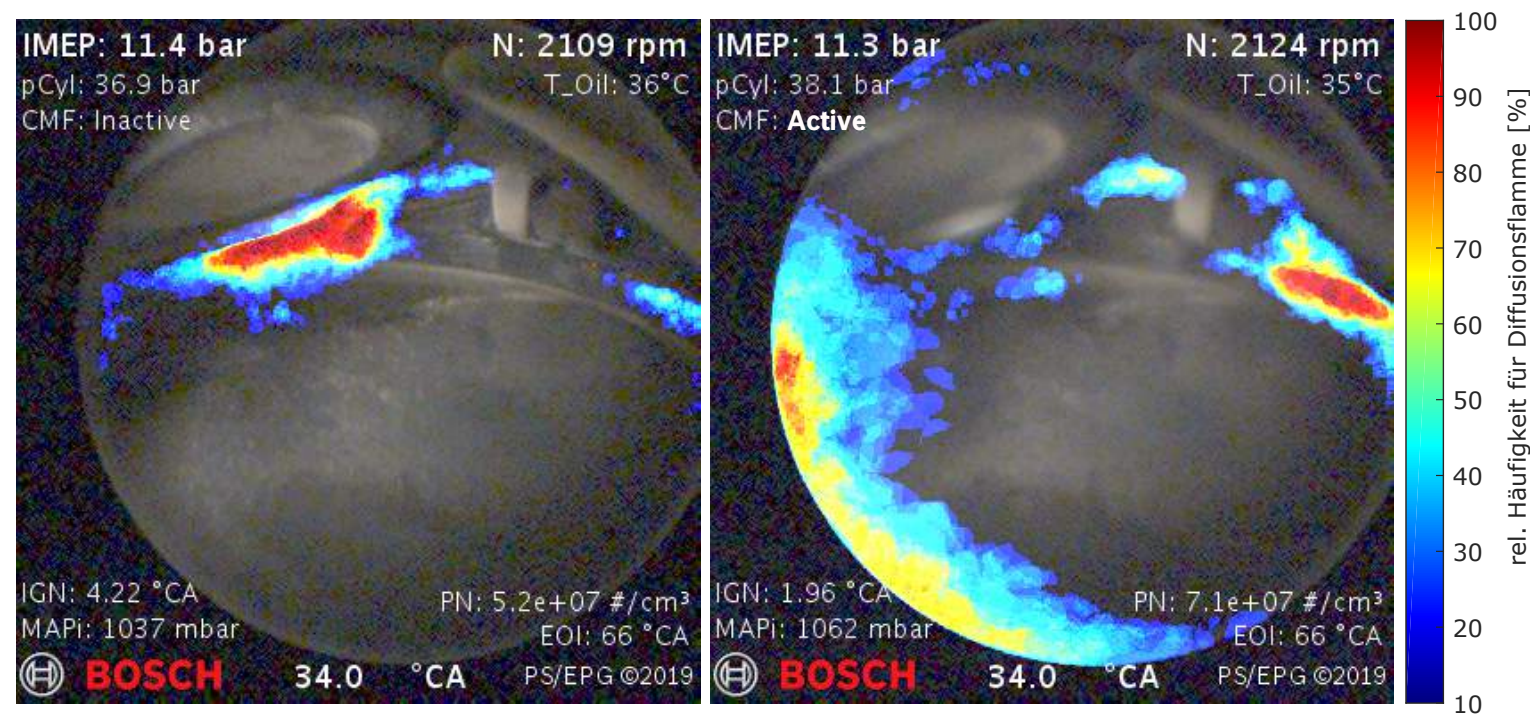

Abbildung 4.34.: Gegenüberstellung der Auftretenswahrscheinlichkeit von Diffusionsflammen bei IMEP = 12 bar mit deaktivierter LBK (links) und aktivierter LBK (rechts) mit Injektor K und Sprayorientierung nach innnen

Spray-Ausrichtung nach innen sind für den Fall von LBK $=0$ auf der linken Bildhälfte gut zu erkennen. Die Mehrzahl der Diffusionsflammen entstehen an der Innenseite der Einlassventile. Eher seltener und mit geringen Ausmaßen werden auch Diffusionsflammen an der Zylinderlaufbahn dokumentiert. Trotz des identischen Targetings zur Innenseite des Einlasskanals kommt es bei aktivierter Ladungsbewegungsklappe zu massiven Diffusionsflammen an der äußeren Zylinderlaufbahn. Es muss also eine starke Ablenkung des Sprays durch die Ansaugströmung vom ursprünglichen Targeting vorliegen.

Die Anstellung der Ladungsbewegungsklappe führt am Motorbetriebspunkt von $\mathrm{N}=2000 \mathrm{~min}^{-1}$ und IMEP $=12$ bar zu einer mehr als doppelt so hohen Strömungs-

${ }^{5} \mathrm{PN}-$ Wert für Injektor K, oVI, LBK $=0$ und Orientierung nach außen liegt bei $1,4 \cdot 10^{8} \mathrm{\#} / \mathrm{cm}^{3}$ 
geschwindigkeit im Einlasskanal als bei $\mathrm{LBK}=0$, wie in Abbildung 4.20 im Teilkapitel 4.2.5 zu sehen ist. Dadurch erfahren die Tropfen des saugsynchron eingespritzten Sprays eine starke Beschleunigung in die Strömungsrichtung der Ansaugströmung und somit eine Ablenkung von ihrer ursprünglichen Flugbahn. Dies ist sowohl für das Single-Spray mit Injektor E, als auch für das Twin-Spray mit Injektor K der Fall. Der entscheidende Unterschied liegt jedoch im Abspritzpunkt, also der Position der Injektorspitze relativ zum Einlasskanal. Bei einer Twin-Konfiguration müssen die Injektoren zwangsläufig links und rechts außen bezüglich des Einlasskanals positioniert werden, während ein Single-Injektor stets mittig platziert werden kann. So führt die Ablenkung der Tropfen durch die Ansaugströmung mit LBK $=1$ beim Single-Injektor E zu einer Konzentration des Sprays an der Kanalinnenseite. Die Auswirkungen auf die resultierenden Diffusionsflammen können in Abbildung A.23 im Anhang als Vergleichsbasis eingesehen werden. Bei den Twin-Sprays mit Injektor K kommt es jedoch zu einer unerwünschten Konzentration der Sprays an den Kanalaußenseiten. Zum besseren Verständnis sind die beschriebenen Ablenkungen der Sprays durch die Ansaugströmung in Abbildung 4.35 skizziert. Die in Kapitel 4.2.5 beschriebenen Sekundärzerstäubungseffekte mit LBK = 1 kommen beim Twin-Spray aufgrund des unmittelbaren Auftreffens der Tropfen an der äußeren Zylinderlaufbahn kaum zur Geltung.
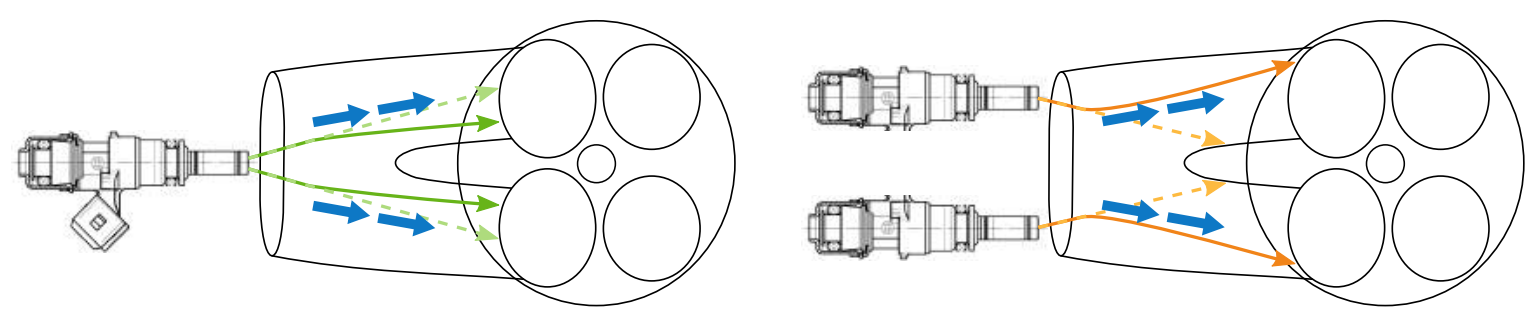

Abbildung 4.35.: Skizzen zur Wechselwirkung der starken Ansaugströmung bei LBK $=1$ mit dem Single-Spray von Injektor E (links) und dem Twin-Spray mit Orientierung nach innen von Injektor K (rechts)

Der mehrfach dokumentierte PN-Vorteil der angestellten Ladungsbewegungsklappe mit Single-Sprays und oVI ist somit anhand von zwei Effekten erklärbar. Zum einen sind nach den Berechnungen in Abbildung 4.20 die Bedingungen für Sekundärzerstäubung im Einlasskanal gegeben, was zu einer Verkleinerung insbesondere der größeren Tropfen führt. Zum anderen ist mit einer Ablenkung des Sprays zur Kanalinnenseite und somit zur Brennraummitte zu rechnen, was sich ebenfalls günstig auf die Gemischbildung auswirkt. Bei Twin-Konfigurationen in Kombination mit einer Ladungsbewegungsklappe ist die Wechselwirkung zwischen Ansaugströmung und Spray-Targeting in besonderem Maße zu berücksichtigen, da die Gefahr einer Laufbahnbenetzung durch die nach außen versetzten Abspritzpunkte erhöht ist. 


\subsubsection{Kraftstoffdruck}

Der Kraftstoffdruck $p_{K r}$ ist für die meisten Kraftstoffsysteme mit Saugrohreinspritzung im Markt kein flexibler, sondern ein vorab fixierter Parameter. Der serientypische Kraftstoffdruck liegt im Bereich von 3,5 - 4,0 bar. Eine Ausnahme stellt das Kraftstoffsystem vom Typ Bosch DECOS dar, welches den Kraftstoffdruck bedarfsgerecht (DEmand COntrolled fuel Supply) zwischen 2,5 und 6,8 bar bereitstellen kann. Bei höherem Kraftstoffdruck kann hierbei mit kleineren resultierenden Tropfen gerechnet werden. In der Literatur ist eine Vielzahl an meist empirisch ermittelten Gleichungen zu finden, mit welchen der mittlere Sauter-Durchmesser SMD in Abhängigkeit weiterer Größen berechnet werden kann. Eine gute Übersicht darüber, sowie die stark vereinfachte Quintessenz daraus, kann dem Werk von Lefebvre [61] entnommen werden.

$$
S M D \propto \Delta p_{K r}^{-d}
$$

Es besteht eine umgekehrte Proportionalität zwischen den Größen SMD und der Druckdifferenz am Injektor, also zwischen Kraftstoffsystemdruck und Luftdruck stromabwärts der Spritzlöcher. Nach Lefebvre nimmt der Exponent $d$ in Abhängigkeit weiterer Größen, wie beispielsweise Viskosität und Oberflächenspannung der Flüssigkeit, unterschiedliche Werte an $(0,275<d<0,440)$. Mit Hilfe eines in Kapitel 3.4 beschriebenen Messsystems wird der Sauter-Durchmesser des Sprays von Injektor E bei verschiedenen Kraftstoffdrücken vermessen. Die ermittelten SMD-Werte sowie eine Extrapolation mittels Gleichung 4.2 mit $d=0,315$ sind im oberen Bereich von Abbildung 4.36 dargestellt. Dabei wird deutlich, dass durch Anhebung des Kraftstoffdruckes, bei nahezu unveränderten Spraywinkeln, eine wesentliche Verkleinerung der Tropfendurchmesser möglich ist.

Da sich kleine Tropfendurchmesser in den vorangegangenen Kapiteln stets vorteilhaft im Hinblick auf die Partikelemission auswirkten, ist es naheliegend den Einfluss des Kraftstoffdrucks dahingehend zu analysieren. Zur statistischen Absicherung werden Mittelwerte aus fünf Messungen und die jeweilige Streubandbreite der PNEmissionen in Abbildung 4.36 dargestellt. Eine Verkleinerung des Tröpfchendurchmessers um $\triangle S M D=8 \mu m$ durch eine Druckerhöhung von zwei auf drei bar, führt bei warmem Motor und vorgelagerter Einspritzung (cVI) zu einer Reduktion der Partikelkonzentration von mehr als $1,1 \cdot 10^{7} \# / \mathrm{cm}^{3}$, also um etwa $73 \%$. Eine abermalige Druckerhöhung von drei auf vier bar ermöglicht eine weitere Partikelreduktion um $66 \%$. Dies unterstreicht den bereits im vorangegangenen Kapitel 4.2.7 vermuteten, starken Zusammenhang zwischen der Tropfengröße, gekennzeichnet durch den SMD, und der Partikelemission bei vorgelagerter Einspritzung. Eine weitere Erhöhung des Systemdrucks von vier auf bis zu 9,5 bar führt in diesem Fall zwar zu einer zusätzlichen, messbaren Reduktion der PN-Emission, jedoch fällt der Gradient hierbei flacher aus.

Bei saugsynchroner Einspritzung (oVI) ist bei warmem Motor kein Einfluss des Kraftstoffdrucks auf die Partikelemissionen zu erkennen. Dies ist insofern plausibel, als dass 

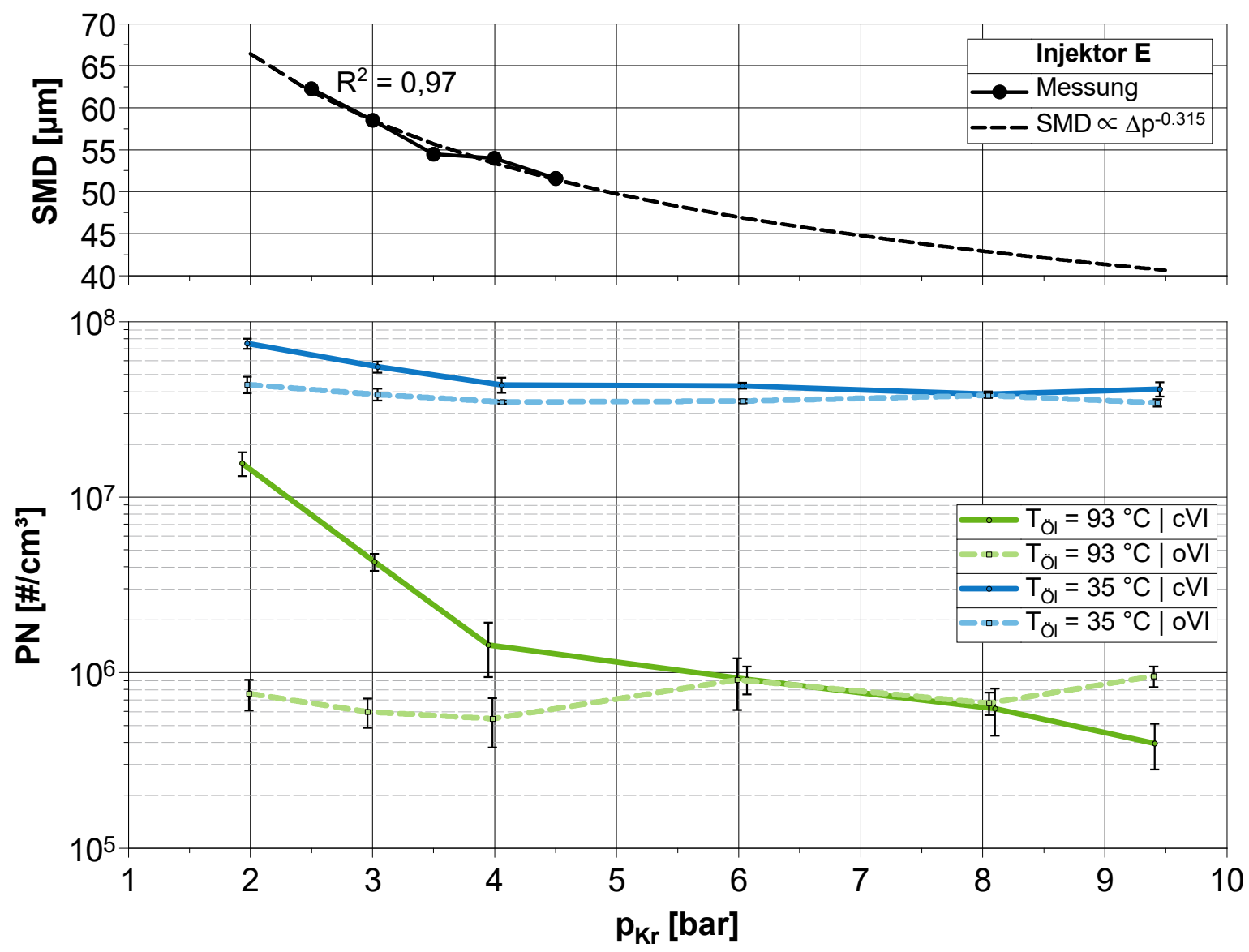

Abbildung 4.36.: Einfluss des Kraftstoffdrucks $p_{K r}$ auf die Tröpfchendurchmesser des Sprays von Injektor $\mathrm{E}$ sowie auf die Partikelemissionen bei $\mathrm{N}=2000 \mathrm{~min}^{-1}, \mathrm{IMEP}=12$ bar und LBK $=0$

die besonders großen Tropfen, welche bei geringem $p_{K r}$ entstehen, eine höhere Wahrscheinlichkeit aufweisen durch Sekundärzerstäubung im Einlassventilspalt oder auch im Einlasskanal zerkleinert zu werden (siehe Gleichung 2.3). Auch die langsameren Austrittsgeschwindigkeiten der Tropfen bei niedrigen Drücken führt zu einer größeren Weber-Zahl, da somit die Relativgeschwindigkeit zwischen Tropfen und Ansaugströmung steigt. Auch bei kaltem Motor und somit deutlich höherem PN-Niveau sind diese Effekte wiederzufinden. Für eine vorgelagerte Einspritzung ist besonders die Systemdruckerhöhung von zwei auf vier bar von Vorteil. Bei saugsynchroner Einspritzung führt eine Druckerhöhung von vier auf 9,5 bar zu keiner messbaren Verbesserung. Lediglich ein geringfügiger Anstieg der PN-Konzentration ist bei zu niedrigen Drücken kleiner vier bar erkennbar.

In Abbildung A.22 im Anhang ist der Einfluss des Kraftstoffdrucks auf die gasförmigen Schadstoffemissionen $\mathrm{HC}, \mathrm{CO}$ und $\mathrm{NO}_{x}$, sowie des Restsauerstoffs $\mathrm{O}_{2}$ veranschaulicht. Auch hierbei handelt es sich um Mittelwerte von jeweils fünf Messungen. Hinsichtlich der HC-Emissionen sind die zuletzt beschriebenen Auswirkungen des Systemdrucks auf 
die Tropfengröße erneut erkennbar. Gerade bei vorgelagerter Einspritzung ermöglichen die kleineren Tropfendurchmesser einen reduzierten Wandfilmaufbau im Bereich der Einlassventilsitze. Dadurch kommt es neben geringerer Partikelentstehung auch zu reduzierten HC-Emissionen. Auch das Verhalten der HC-Emissionen bei saugsynchroner Einspritzung deckt sich mit dem der Partikelemissionen, da auch hier kein Einfluss des Kraftstoffdrucks messbar ist. Deutlich größere Kraftstoffwandfilme bei kaltem Motor haben (analog zu PN) entsprechend höhere HC-Konzentrationen zur Folge.

Im Bezug auf die $C O$-Emissionen ist jedoch keine konsistente Korrelation zum Kraftstoffdruck erkennbar. Insofern zeigt der Systemdruck keinen nennenswerten Einfluss auf die Vollständigkeit der Verbrennung. Dies gilt sowohl für vorgelagertes als auch für saugsynchrones Einspritztiming. Der Restsauerstoffgehalt $\mathrm{O}_{2}$, ein Indikator für die Qualität der Homogenisierung des Luftkraftstoffgemisches, ist ebenfalls weitestgehend unabhängig vom Systemdruck. Auch bezüglich der $N O_{x}$-Emissionen scheint kein eindeutiger Zusammenhang zum Kraftstoffdruck zu bestehen. Lediglich bei kaltem Motor ist ein geringfügiger Anstieg der $\mathrm{NO}_{x}$-Konzentration für sehr kleine Kraftstoffdrücke zu verzeichnen. Neben dem ebenfalls geringen Anstieg des Restsauerstoffs $\mathrm{O}_{2}$ bei dieser Parameterkombination stellt die Bildung von Prompt- $N O$ einen möglichen Erklärungsansatz hierfür dar. Bei diesem Reaktionsmechanismus entsteht Stickstoffmonoxid bereits bei relativ niedriger Temperatur und bevorzugt in brennstoffreichen Zonen direkt in der Flammenfront. Diese Bedingungen sind in den Diffusionsflammen in unmittelbarer Nähe zu Kraftstoffwandfilmen gegeben. Das allgemein höhere $N O_{x^{-}}$ Niveau bei kaltem Motor könnte somit teilweise auf die ausgeprägteren Wandfilme, welche die Bildung von Prompt-NO begünstigen und auf die höhere Konzentration des Restsauerstoffs $\mathrm{O}_{2}$ zurückzuführen sein. Nach vergleichenden Berechnungen der Brennverläufe und der maximalen Temperaturen im Brennraum $T_{Z y l, \max }$ ist hervorzuheben, dass nahezu dieselben Spitzentemperaturen bei warmem und kaltem Motor erreicht werden. Eine unterschiedlich stark ausgeprägte Bildung von thermischem NO bei warmem und kaltem Motor kann somit ausgeschlossen werden. Die geringere Ausgangstemperatur bei kaltem Motor, sowie die höheren Wandwärmeverluste, können durch deutlich frühere Zündwinkel, höhere Spitzendrücke und folglich frühere Verbrennungsschwerpunktlagen (MFB $50 \%=11^{\circ} \mathrm{KW}$ ) ausgeglichen werden. Bei warmem Motor muss der Zündwinkel aufgrund von Klopfen nach spät verschoben werden, was zu deutlich späterer Schwerpunktlage führt (MFB $50 \% \geq 25^{\circ} \mathrm{KW}$ ).

\subsubsection{Potenzial einer SRE-Mehrfacheinspritzung}

In den Kapiteln 4.2.3 bis 4.2.6 wird an mehreren Stellen deutlich, dass sich die PartikelQuellen für vorgelagerte und saugsynchrone Einspritzungen unterscheiden. Während bei vorgelagertem Timing Diffusionsflammen vorwiegend an den Einlassventilsitzen entstehen, spielt die Benetzung der Zylinderlaufbuchse bei saugsynchroner Einspritzung eine dominierende Rolle. Dies soll bei einer Zweifacheinspritzung ausgenutzt werden. Dabei erfolgt die erste Einspritzung stets vorgelagert, die zweite möglichst saug- 
synchron. Der Grundgedanke besteht hierbei darin, dass zwei kleinere Wandfilme an verschiedenen Orten jeweils besser verdunsten können, als ein größerer Kraftstoffwandfilm. Für die Partikelentstehung wäre dies dann von Vorteil, wenn bis zur jeweiligen Erfassung der Wandfilme durch die Flammenfront eine geringere Kraftstoffmasse in flüssiger Form vorläge. Ob sich dieses Gedankenmodell in der Praxis wiederfindet, zeigen die Auswertungen in Abbildung 4.37. Auf der Ordinate ist der Massenanteil
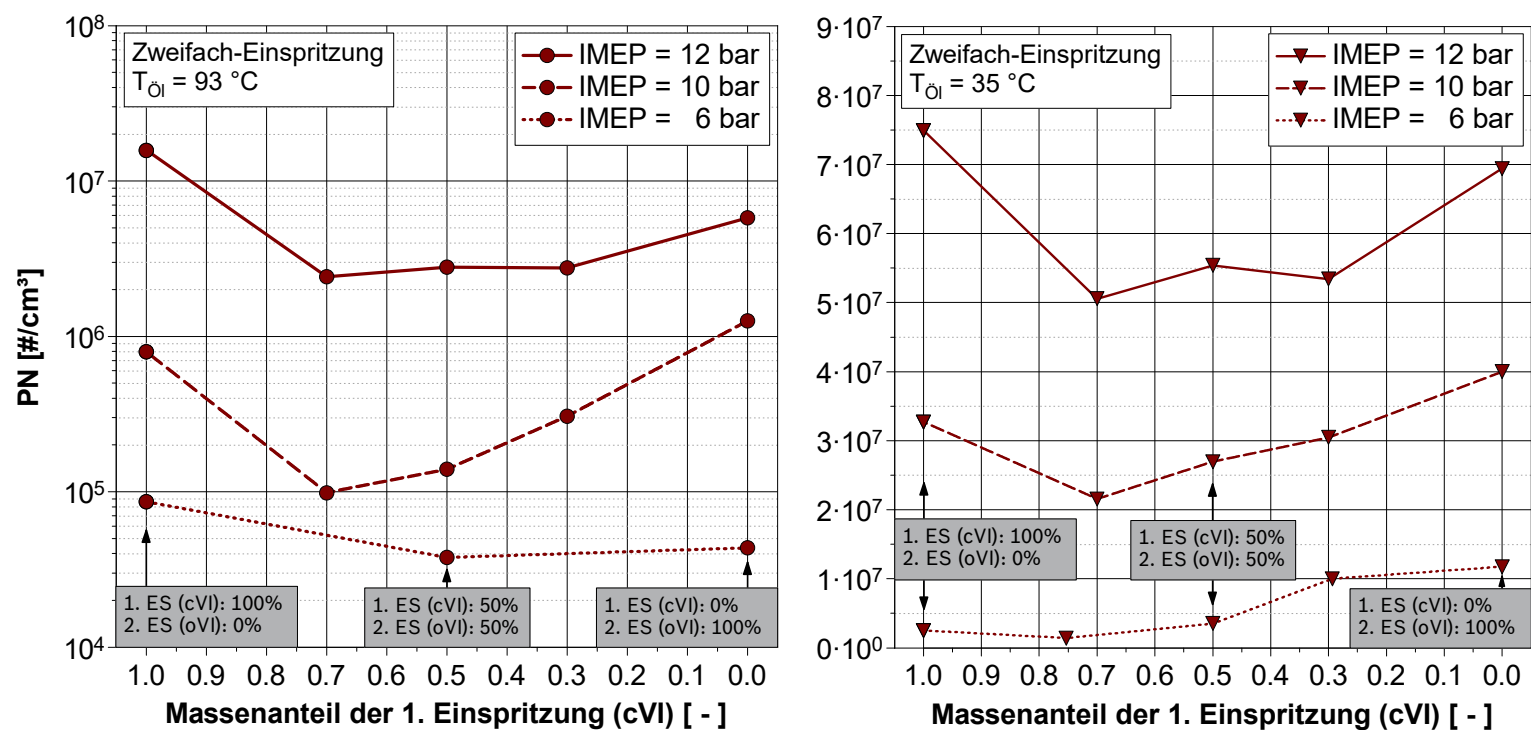

Abbildung 4.37.: Partikelemissionen bei Variation des Massenaufteilungsfaktors einer Zweifach-Einspritzung bei warmem Motor (links) und kaltem Motor (rechts) mit Twin-Injektor J orientiert nach oben

der ersten, vorgelagerten Einspritzung (cVI) aufgetragen. Folglich befinden sich ganz links bei Faktor 1,0 reine vorgelagerte Einfacheinspritzungen. Weiter rechts steigt der Massenanteil der zweiten, saugsynchronen Einspritzung. Der Massenanteil der ersten Einspritzung sinkt daher gleichermaßen. Ganz rechts bei Faktor 0,0 befinden sich somit Messungen mit reiner saugsynchroner Einfacheinspritzung.

Gerade bei höheren Motorlasten wird bei warmem und kaltem Motor deutlich, dass eine Zweifach-Einspritzung mit einer Massenaufteilung von $70 \%$ vorgelagert und $30 \%$ saugsynchron eine Reduktion der Partikelemissionen gegenüber den jeweiligen Einfacheinspritzungen ermöglicht. Bei warmem Motor sind so Verbesserungen von etwa $50 \%$ bei IMEP $=12$ bar und bis hin zu $85 \%$ bei IMEP $=10$ bar darstellbar. Bei kaltem Motor kann immerhin eine PN-Reduktion um ca. $30 \%$ für beide oberen Mitteldrücke erreicht werden. Das Konzept einer SRE-Zweifacheinspritzung zeigt demnach Wirkung. 


\section{Zusammenfassung}

Die vorliegende Arbeit umfasst experimentelle Untersuchungen zu den Ursachen der Partikelentstehung in Ottomotoren mit Saugrohreinspritzung, sowie die Ableitung möglicher Maßnahmen zur Verringerung der Partikelemission. Einen besonderen Schwerpunkt stellt in dieser Arbeit die Gemischbildung dar. Für grundlegende Untersuchungen der Gemischbildung im Einlasskanal werden Verdunstungsmessungen an einer eigens dafür entwickelten Kammer in der Gestalt eines realen Einlasskanals durchgeführt. Der Einfluss der Gasdynamik auf die Gemischbildung im Einlasskanal wird anhand von Ladungswechselrechnungen auf Basis von Messungen an einem 4-Zylinder Vollmotor analysiert. Des Weiteren kommen bildgebende Verfahren, sowohl mittels Endoskopie am Vollmotor, als auch an einem geschleppten Transparentmotor mit besserer optischer Zugänglichkeit zum Einsatz.

Mit Hilfe der High-Speed Video-Endoskopie am Vollmotor konnten die Entstehungsorte der Partikel anhand von Diffusionsflammen lokalisiert und die Wechselwirkung verschiedener Einflussgrößen untersucht werden. Unter Verwendung der MathematikSoftware MATLAB wurden im Rahmen dieser Arbeit Auswertungsalgorithmen entwickelt, welche die Detektion von Diffusionsflammen und eine statistische Bewertung derselben ermöglichen. Eine tiefer gehende Analyse von Kraftstoffwandfilmen im Brennraum, welche häufig die Hauptursache für die Partikelentstehung darstellen, erfolgt mittels High-Speed Kameras am Transparentaggregat. Mit Hilfe eines weiteren Bildverarbeitungsalgorithmus gelang die Erkennung von flüssigen Wandfilmen, sowohl auf der Unterseite der Einlassventile, als auch an der Zylinderlaufbuchse am Transparentmotor. Eine quantitative Bewertung der Wandfilme erfolgte anhand der ermittelten Wandfilmfläche, sowie der statistischen Auftretenswahrscheinlichkeit. Durch die Verwendung des gleichen Zylinderkopfes, sowohl am Transparentmotor als auch am Vollmotor und durch die Übertragung der Einlasskanalgeometrie dieses Zylinderkopfes auf die Verdunstungskammer, ist eine Übertragbarkeit der Erkenntnisse aus den jeweiligen Experimenten gewährleistet.

Im Rahmen der verschiedenen Parameterstudien der vorliegenden Arbeit konnten mit Hilfe der High-Speed Video-Endoskopie drei dominante Quellen für die Partikelentstehung innerhalb des Brennraums identifiziert werden. Diese sind in Abbildung 5.1 zusammengefasst. Kraftstoffwandfilme, welche die Hauptursache für die Partikelentstehung an den Einlassventilen sowie an der Zylinderlaufbuchse darstellen, konnten durch Messungen am geschleppten Transparentmotor nachgewiesen und anhand ihrer Fläche und Auftretenswahrscheinlichkeit bewertet werden. Bei dem für die Saugrohreinspritzung typischen, frühen Einspritzzeitpunkt, welcher eine Vorlagerung des 


\begin{tabular}{|c|c|l|}
\hline PN Quelle & Visualisierung & \multicolumn{1}{|c|}{ Entstehungsmechanismus } \\
\hline $\begin{array}{c}\text { Einlassventile } \\
\& \\
\text { Ventilsitzspalte }\end{array}$ & & $\begin{array}{l}\text { Kraftstoffwandfilme } \\
\text { - durch Filmtransport aus dem Einlasskanal } \\
\text { - aus direkter Benetzung } \\
\text { Vorwiegend bei vorgelagerter ES (cVI) }\end{array}$ \\
\hline Laufbuchse & & $\begin{array}{l}\text { Kraftstoffwandfilme } \\
\text { - unterhalb der Auslassventile } \\
\text { - seitlich der Einlassventile } \\
\text { Vorwiegend bei saugsynchroner ES (oVI) }\end{array}$ \\
\hline Gasphase & & $\begin{array}{l}\text { Lokal fette Zonen } \\
\text { - durch mangelhafte Homogenisierung } \\
\text { - durch unvollständige Tropfenverdunstung } \\
\text { Vorwiegend bei geringer Ladungsbewegung }\end{array}$ \\
\hline
\end{tabular}

Abbildung 5.1.: Übersicht der drei dominierenden Partikelquellen innerhalb des Brennraums mit Saugrohreinspritzung

Kraftstoffes an den Einlassventilen zur Folge hat, stellen die Einlassventile sowie die Einlassventilsitze die häufigste Quelle für die Partikelentstehung dar. Bei hoher Motorlast kann eine spätere Einspritzung in die offenen Einlassventile, sowohl im Hinblick auf den Kraftstoffverbrauch, als auch auf die Schadstoffemission, sinnvoll sein. Bei dieser saugsynchronen Einspritzstrategie wurden jedoch vielfach Diffusionsflammen im oberen Bereich der Zylinderlaufbuchse dokumentiert, je nach Auslegung der Spraywinkel im Bereich unterhalb der Auslassventile, oder auch seitlich der Einlassventile. Neben einer direkten Benetzung dieser Zonen ist eine Akkumulation von Kraftstoffwandfilmen durch die Aufwärtsbewegung des Kolbens möglich. Eine weitere Form der Partikelentstehung ist durch Zonen mit lokalem Sauerstoffmangel in der Gasphase gegeben. Als Ursachen kommen hierbei eine mangelhafte Homogenisierung oder unvollständig verdunstete, meist große, Kraftstofftropfen in Frage.

Obwohl die Bedingungen für die Partikelentstehung hinsichtlich der Temperatur und des lokalen Luft-Kraftstoff-Verhältnisses für alle Ottomotoren allgemeingültig sind, unterscheiden sich die kritischen Stellen im Brennraum zwischen Motoren mit Saugrohreinspritzung (SRE) und mit Direkteinspritzung (BDE) dennoch. Dieser Sachverhalt wird durch eine Bewertung der Partikelquellen im Brennraum in Abbildung 5.2 bei kaltem und warmem Motor für beide Brennverfahren verdeutlicht. Während bei der Saugrohreinspritzung die Einlassventile, die Einlassventilsitze und die Zylinderlaufbahn die häufigsten Entstehungsorte darstellen, steht bei der Benzindirekteinspritzung meist der Kolben sowie die Injektorspitze im Fokus [57]. Bei SRE besteht ein wichtiger, 

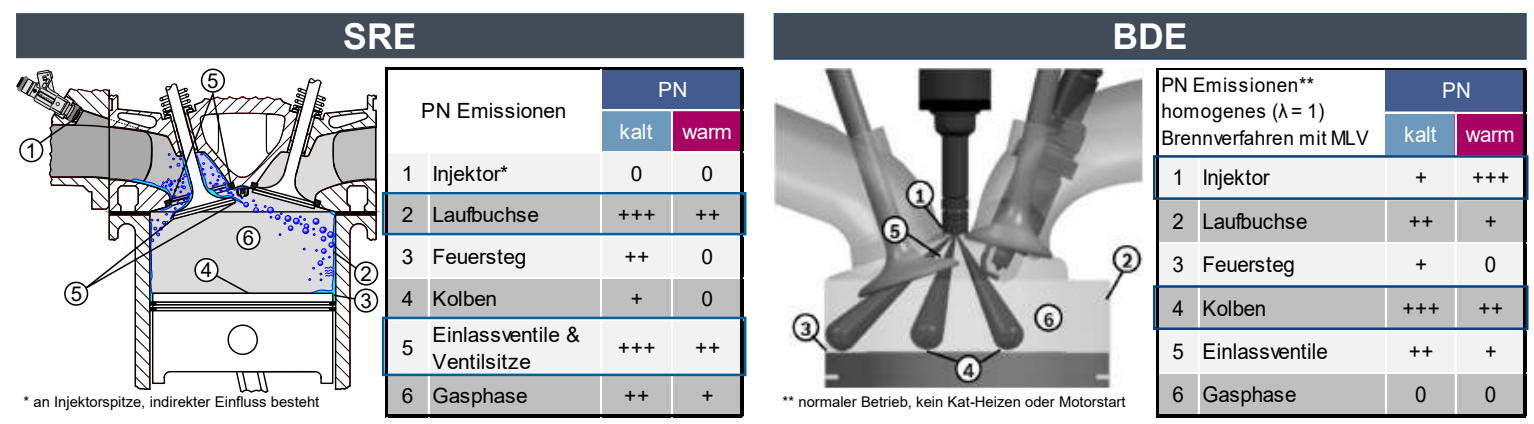

Abbildung 5.2.: Bewertung der PN-Quellen mit Saugrohreinspritzung [106] und Gegenüberstellung zur Direkteinspritzung nach Kufferath et al. [57].

$0 \hat{=}$ geringer Einfluss; $+++\hat{=}$ großer Einfluss

aber indirekter Einfluss des Injektors auf die Partikelemission, da die Injektorspitze nicht in den Brennraum ragt. Dagegen stellt bei der Direkteinspritzung die durch die Einspritzung nahezu unvermeidliche Benetzung der Injektorspitze und die daraus entstehende Belagsbildung eine komplexe technische Herausforderung dar [18, 56]. In jüngster Zeit konnten hierbei jedoch beachtliche Fortschritte erzielt werden [19, 82].

Ein Großteil der vorliegenden Arbeit widmet sich der Analyse verschiedener Einflussgrößen auf die Partikelemission. Bei Ottomotoren mit Saugrohreinspritzung sind die Auswirkungen des Parameters Motorlast, beziehungsweise des entsprechenden Saugrohrdrucks, auf die Gemischbildung von essenzieller Bedeutung für das Verständnis der Partikelentstehung im Brennraum. Auch mit Hilfe der bildgebenden Verfahren konnte gezeigt werden, dass die in der Literatur beschriebene Rückströmung [111, 121] besonders bei geringem Saugrohrdruck einen positiven und wesentlichen Beitrag der Gemischbildung darstellt. Im Kontext der Partikelentstehung kommt der Zurückdrängung von Kraftstoffwandfilmen in den Einlasskanal durch die Rückströmung eine wichtige Bedeutung zu, weil dadurch kritische Wandfilme im Brennraum vermieden oder reduziert werden können. Die Messungen dieser Arbeit zeigten weiterhin, dass mit steigender Last, und folglich höherem Saugrohrdruck, die unterstützende Wirkung der Rückströmung kontinuierlich abnimmt, und bei Motoren mit Aufladung letztendlich nahezu verschwindet. Hohe Partikelemissionen aufgrund des Transports von Kraftstoffwandfilmen aus dem Einlasskanal in den Brennraum sind die Folge.

Untersuchungen mit Variationen des Einspritztimings ergaben Optima hinsichtlich der PN-Emission, welche stark vom Motorbetriebspunkt abhängig sind. Eine Ausnutzung der intensiven Rückströmung bei geringer Motorlast gelingt am besten mit früher, vorgelagerter Einspritzung (closed Valve Injection, cVI). Bei hoher Motorlast kann eine spätere Einspritzung, welche anteilig saugsynchron erfolgt, von Vorteil sein. Eine wichtige Erkenntnis in diesem Zusammenhang wurde durch Analysen mit Hilfe der High-Speed Video-Endoskopie erarbeitet. Trotz gleicher gemessener Partikelkonzentration beim Vergleich von vorgelagerter und saugsynchroner Einspritzung war dies auf gänzlich verschiedene Partikelquellen im Brennraum zurückzuführen. Bei der Suche nach Optimierungsansätzen ist das von großer Bedeutung. So gilt es bei vorgelagerter 
Einspritzung vorwiegend die Wandfilmbildung an den Einlassventilen zu reduzieren, während bei saugsynchroner Einspritzung der Wandkontakt der Spraytropfen mit der Zylinderlaufbahn zu vermeiden ist.

Eine Erhöhung der Ladungsbewegung mit Hilfe einer Ladungsbewegungsklappe hat sich im Rahmen dieser Arbeit als ausgesprochen wirkungsvolle Maßnahme hinsichtlich der Reduktion der Partikelemission erwiesen. Die hierdurch erreichte Verbesserung der Gemisch-Homogenisierung kann die Entstehung von Diffusionsflammen in der Gasphase deutlich mindern. Durch Berechnungen der Weberzahl für Spraytropfen $W e_{T r}$, sowie des kritischen Tropfendurchmessers $d_{k r i t}$. konnte außerdem gezeigt werden, dass eine angestellte Ladungsbewegungsklappe Sekundärzerstäubung im Einlasskanal ermöglichen kann. Eine deutlich höhere Strömungsgeschwindigkeit der Ansaugluft, induziert durch die Querschnittsverengung einer angestellten Klappe, führt demnach zur Zerkleinerung aller Tropfen mit $d_{T r} .>d_{k r i t}$.

Im Rahmen zweier Spray-Targeting Studien wurde der Einfluss der Spraywinkel auf die Partikelemission analysiert. Da veränderte Spraywinkel nicht nur das Benetzungsverhalten eines Injektors im Einlasskanal, sondern auch stets die Tropfenaufbereitung und somit das Tropfengrößenspektrum beeinflussen, ist die Interpretation dieser Versuche nicht trivial. Dennoch konnte im Rahmen einer Targeting-Studie mit SingleKonfigurationen ermittelt werden, dass sich weit aufgefächerte Einzelspraykeulen, also große $\beta_{80}$-Winkel (Winkeldefinitionen siehe Abbildung 2.2b), allgemein vorteilhaft hinsichtlich einer Partikelreduktion auswirken. Die Vorzüge von weit aufgefächerten, breiten Sprays hinsichtlich der Partikelemission sind nach Messungen von Claret et al. [15] und Petit et al. [83] auch auf SRE-Motoren ohne Aufladung übertragbar. Eine Kombination von kompakten Spraykeulen, gekennzeichnet durch kleine $\beta_{80}$-Winkel, mit großen $\alpha_{50}$-Winkeln hat sich in der vorliegenden Arbeit als besonders ungünstig erwiesen, und muss vermieden werden. Kleine $\beta_{80}$-Winkel sind im Hinblick auf Akkumulation von Kraftstoffwandfilmen im Einlassventilspalt zwar immer kritisch zu bewerten, in Kombination mit großen $\alpha_{50}$-Winkeln steigt jedoch das Risiko, dass flüssiger Kraftstoff rasch an die Zylinderlaufbahn jeweils seitlich der Einlassventile gelangt.

Ein Versuch zur unabhängigen Bewertung der Einflussgrößen mittlerer Tropfendurchmesser (SMD) und Spray-Targeting wurde anhand einer Twin-Targeting-Studie angestellt. Durch symmetrische Rotation der mit einem $\gamma$-Winkel aufgebauten Injektoren in ihrer Einbaulage konnte das globale Spray-Targeting bei konstantem SMD variiert werden. Für vorgelagerte Einspritztimings sind die Auswirkungen der TargetingÄnderungen durch die Injektor-Rotation bemerkenswert gering. Bei saugsynchroner Einspritzung spielt das globale Targeting hingegen eine entscheidende Rolle. Hierbei konnte bestätigt werden, dass eine Ausrichtung des Sprays an die Kanalaußenseiten unbedingt zu vermeiden ist. Eine Fokussierung der Spraymasse zur Kanalinnenseite, und somit zur Brennraummitte, erwies sich als zielführend. Versuche mit verschiedenen Sprays mit unterschiedlichen $\alpha_{80}$-Winkeln und unterschiedlichem SMD lieferten Indizien, dass bereits geringfügig kleinere SMD-Werte eines Sprays bei vorgelagerter Einspritzung erhebliche PN-Reduktionen ermöglichen. 
Im Rahmen von Versuchen zur Variation des Kraftstoffdrucks, welcher sich unmittelbar auf den Sauter-Durchmesser eines Sprays auswirkt [61], konnte dieser Zusammenhang bestätigt werden. Eine Verringerung des SMDs um lediglich $8 \mu m$ durch eine Druckerhöhung von zwei bar auf drei bar führte bei warmem Motor und vorgelagerter Einspritzung (cVI) zu einer Reduktion der Partikelkonzentration um etwa $73 \%$. Bei saugsynchroner Einspritzung ist der Einfluss der Tropfengröße und somit auch des Kraftstoffdrucks deutlich geringer. Es kann jedoch nicht ausgeschlossen werden, dass die Druckerhöhung neben der Verringerung des Sauterdurchmessers weitere Effekte zur Folge hat, welche die Gemischbildung positiv beeinflussen. Es besteht beispielsweise die Möglichkeit, dass bei Tropfen-Wand-Interaktionen, aufgrund des veränderten Tropfenimpulses und der sich minimal verändernden Spraywinkel, kleinere Sekundärtropfen erzeugt werden. Eine völlig isolierte Bewertung des Parameters Tropfendurchmesser hinsichtlich der Partikelemission ist insofern schwierig.

Durch die Applikation einer Zweifach-Einspritzung, wobei die erste Einspritzung vorgelagert und die zweite bewusst saugsynchron erfolgt, gelang es die Kenntnis der jeweils unterschiedlichen Partikelentstehungsorte auszunutzen. Durch die Aufteilung der Kraftstoffmasse auf zwei Wandfilme an unterschiedlichen Stellen, den Einlassventilen und der Zylinderlaufbahn, konnte eine Akkumulation an einem Ort mit besonders langer Verdunstungsdauer vermieden werden. Besonders bei warmem Motor ist das Reduktionspotenzial der Partikelemission von bis zu $85 \%$ hervorzuheben.

Da beim Kaltstart und beim Katalysatorheizen im Leerlauf, aufgrund des rasch abfallenden, beziehungsweise niedrigen Saugrohrdrucks, relativ geringe Partikelemissionen zu verzeichnen waren, wäre für künftige Untersuchungen die Nachstellung einer Beschleunigung während des Motorwarmlaufs hinsichtlich der Partikelemission interessant. Eine Steigerung der Motorlast unmittelbar nach dem Kaltstart, mit gegebenenfalls späten Zündzeitpunkten, stellt ein aus Emissionssicht kritisches, aber hinsichtlich der Emissionen bei Messungen im Straßenverkehr (EU6-RDE) relevantes und zu optimierendes, Fahrmanöver dar. Des Weiteren steht die Bewertung des Einflusses eines Partikelfilters auf die Gemischbildung mit Saugrohreinspritzung noch aus. Es ist aufgrund der anzunehmenden Steigerung des Abgasgegendruckes eine leichte Wirkungsgradverschlechterung zu erwarten. Die in dieser Arbeit beschriebene Abgasrückströmung beim Öffnen der Einlassventile aus dem Brennraum zurück in den Einlasskanal könnte sich durch den höheren Abgasgegendruck jedoch möglicherweise leicht verstärken und die Gemischbildung sogar positiv beeinflussen. Letztlich gilt es die in dieser Arbeit vorgestellten Maßnahmen zur Verringerung der PN-Emission durch Fahrzeugmessungen, sowohl anhand von Fahrzyklusmessungen auf dem Rollenprüfstand, als auch mittels portabler Emissionsmesstechnik (PEMS) bei realen Fahrten auf der Straße (RDE) zu validieren. Aufgrund der großen Sensitivität der Parameter Motortemperatur und Last auf die Partikelemission ist es jedoch fraglich, ob insbesondere bei SRE-Motoren mit Aufladung rein innermotorische Maßnahmen zur Reduktion der PN-Emission ausreichen werden, um künftigen Abgasgesetzgebungen gerecht zu werden. Dynamische Abfahrten bei kaltem Motor (z.B. $T_{0}=0{ }^{\circ} \mathrm{C}$ ) bei einer RDE-Fahrt machen die Implementierung eines Partikelfilters möglicherweise unumgänglich. 


\section{A. Anhang}

\section{A.1. Exkurs - Kalt-Start \& Katalysatorheizen}

Da während des Motorstarts und des Katalysatorheizbetriebs eine Konvertierung der Schadstoffemissionen $\mathrm{HC}, \mathrm{CO}$ und $N O_{x}$ im Katalysator noch nicht möglich ist, müssen in dieser frühen Phase des Motorwarmlaufs auch die gasförmigen Rohemissionen minimiert werden. Ein weiteres Ziel der Untersuchung von Kalt-Start und Katalysatorheizbetrieb bei $T_{0}=20^{\circ} \mathrm{C}$ ist die Identifikation der Einflussgrößen auf die Partikelemission, um mögliche Verbesserungsmaßnahmen abzuleiten.

Zur Untersuchung dieser Sonderbetriebsarten wird der Versuchsaufbau am Vollmotorprüfstand erweitert, um den Anforderungen des hochdynamischen Startvorgangs gerecht zu werden. Eine Kupplung zwischen dem Schwungrad des Motors und der Abtriebswelle zur Prüfstandsbremse sorgt im geöffneten Zustand für einen realistischen Motorhochlauf, der mit einem Motorstart im Fahrzeug vergleichbar ist. Weiterhin wird die Konditionierung von Motoröl und Kühlwasser so erweitert, dass eine Umwälzung und Kühlung auch bei stehendem Motor $\left(\mathrm{N}=0 \mathrm{~min}^{-1}\right)$ möglich ist. Zusätzlich zum bisherigen Indiziersystem, welches sämtliche Kanäle auf KurbelwinkelBasis erfasst, kommt ein weiteres Indiziersystem zur hochauflösenden Messdatenerfassung auf Zeit-Basis zum Einsatz. Im Hinblick auf die Abgasmesstechnik wird ein Fast-Flame-Ionisiation-Detector (FFID) der Firma Testa zur schnellen Erfassung der unverbrannten Kohlenwasserstoffe (HC) stromabwärts der Turbine und stromaufwärts des Katalysators installiert.

Es wird eine Vorgehensweise angewandt, welche eine vollständige Abkühlung des Motors in möglichst kurzer Zeit nach einer abgeschlossenen Messung von Motorstart und Katalysatorheizen gewährleistet. Dabei gilt es nicht nur die Abkühlung von Motoröl und Kühlwasser, sondern auch die Temperatur sämtlicher Bauteile und Oberflächen, abzuwarten. Hierbei besteht somit ein wesentlicher Unterschied zu den bisher als kalt deklarierten, zwangsgekühlten Messungen. Bei letzteren muss davon ausgegangen werden, dass Brennraumoberflächen und Bauteile wie Einlassventile oder Kolben deutlich wärmer sind als die eingestellte Öl- und Wassertemperatur. Zur Verbesserung der Reproduzierbarkeit wird der Motor vor jeder Messung manuell stets in dieselbe Position gedreht und im Anschluss an jede Messung warm gefahren. Unmittelbar nach dem Kaltstart wird der Betriebspunkt des Katalysatorheizbetriebs von $N=1300 \mathrm{~min}^{-1}$ und IMEP $=2,0$ bar angefahren, um die Schadstoffemission während des realen Motorwarmlaufs zu bewerten. 
Der Kaltstart eines Motors stellt während der ersten Zyklen mit Verbrennung, selbst bei moderater Temperatur von $20^{\circ} \mathrm{C}$, eine große Herausforderung für die Gemischbildung dar. Da aufgrund der kalten Kanal- und Brennraumwände nur ein Bruchteil der eingespritzten Kraftstoffmasse verdunsten kann, ist eine Anreicherung des Gemisches durch eine Mehrmenge erforderlich. Für die Injektorsprays B, E und G und für zweierlei Einspritztimings wird die jeweils geringste, aber mindestens erforderliche, Anreicherung (Anreicherungsfaktor fst) ermittelt, welche erwartungsgemäß die niedrigsten Schadstoffemissionen ermöglicht. Obwohl während der ersten Verbrennungen des Motorhochlaufs stark ausgeprägte Diffusionsflammen zu verzeichnen sind wie Abbildung A.1 zeigt, wird in einem Bilanzzeitraum von sechs Sekunden während des Starts nur eine geringe Partikelanzahl emittiert. Der rasch fallende Saugrohrdruck so-

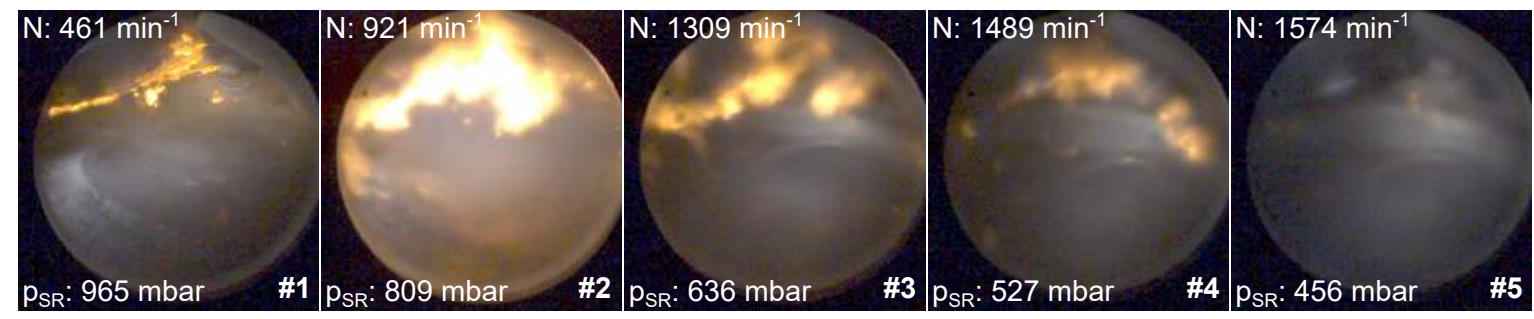

Abbildung A.1.: Die ersten fünf Verbrennungen von Zylinder vier während eines Kaltstarts mit saugsynchroner Einspritzung und Injektor B bei fst $=2,4$

wie die steigende Motordrehzahl ermöglichen bereits nach fünf gefeuerten Arbeitsspielen pro Zylinder Verbrennungen ohne sichtbare Diffusionsflammen mit folglich geringen Partikelanzahlemissionen. Die Notwendigkeit gezielter Anpassungen seitens des Spraytargetings ist somit in Frage zu stellen. Hinsichtlich der Einspritzstrategie ist im Start eine vorgelagerte Einspritzung zu bevorzugen. Saugsynchrone Einspritzungen führen zu höheren PN- und HC-Emissionen, da höhere Anreicherungsfaktoren erforderlich sind.

Bei der ungünstigsten Kombination von Injektor B und saugsynchroner Einspritzung wird mit dem dabei notwendigen Anreicherungsfaktor von fst $=2,4$ im Bilanzzeitraum des Kaltstarts eine Partikelanzahl von 2, 9·10 ${ }^{11}$ \# emittiert. Bezüglich der Fahrstrecke des Testzyklus WLTC von 23,3 km entspricht dies $1,25 \cdot 10^{10} \# / \mathrm{km}$ und damit lediglich $2,08 \%$ vom Grenzwert. Im besten Fall, bei Einsatz von Injektor G und vorgelagerter Einspritzung, beträgt der Anteil der im Start emittierten PN weniger als 0,2\% vom Grenzwert. Die Bandbreite aller in dieser Studie ermittelten Schadstoffemissionen in Bezug zum EU6d-Grenzwert ist in Tabelle A.1 zusammengefasst. Die beim Kaltstart entstehenden Emissionen sind demnach verhältnismäßig gering. Eine Fokussierung der Entwicklungsarbeit auf die Schadstoffreduktion bei anderen Betriebszuständen, wie zum Beispiel den ersten Beschleunigungen mit kaltem Motor, ist daher zu empfehlen.

Bei der Betrachtung der Sonderbetriebsart Katalysator-Heizen im Leerlauf während des Motorwarmlaufs unmittelbar nach einem Kalt-Start fallen die Partikelanzahlemissionen unabhängig vom Spraylayout ebenfalls unkritisch niedrig aus $(<1 \%$ des 


\begin{tabular}{ccccc} 
& PN & HC & CO & NOx \\
\hline "Worst-Case" & $2,08 \%$ & $7,14 \%$ & $4,84 \%$ & $1,94 \%$ \\
Optimierung & $0,15 \%$ & $4,20 \%$ & $2,17 \%$ & $0,95 \%$
\end{tabular}

Tabelle A.1.: Schadstoffemissionen im Bilanzzeitraum des Kaltstarts von sechs Sekunden bezüglich der EU6d-Grenzwerte (Bezugsstrecke WLTC)

EU6-Grenzwerts im WLTC). Als Hauptursache für die geringen PN-Werte, trotz des unvollendeten Motorwarmlaufs, kann der geringe Saugrohrdruck und die damit verbundene intensive Rückströmung identifiziert werden. In Anbetracht von signifikanten HC-Emissionen besteht aber durchaus Optimierungsbedarf beim Betriebspunkt des Katalysatorheizens, wie Abbildung A.2 zeigt. Da sich für die CO-Emissionen, welche

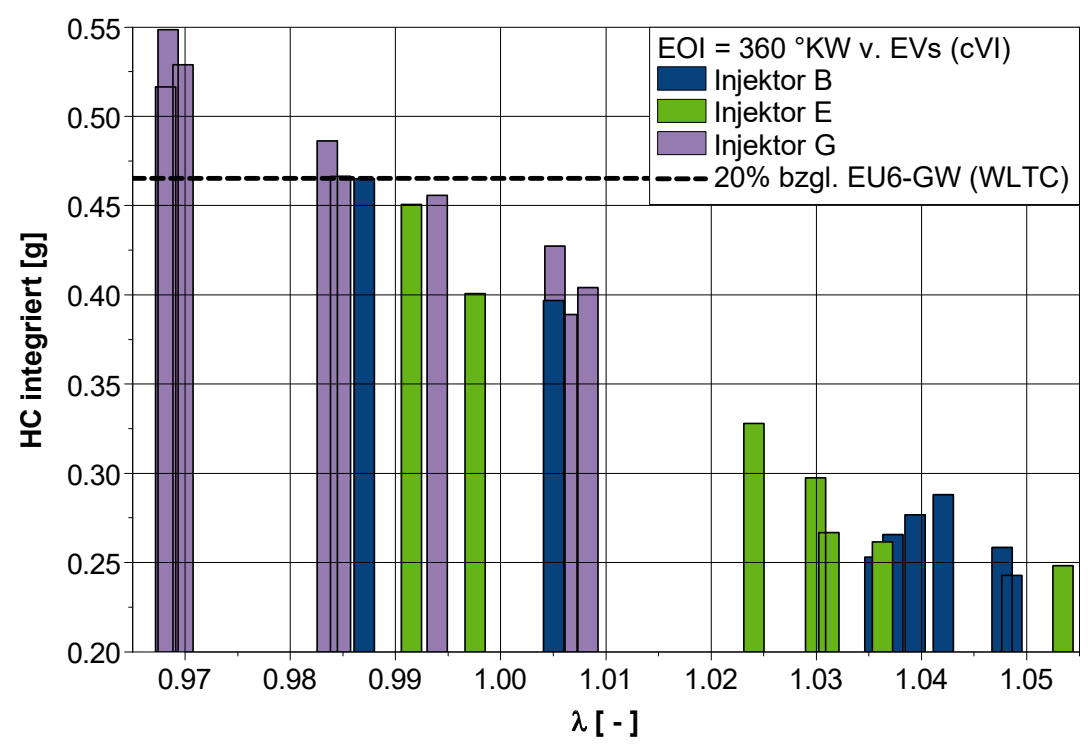

Abbildung A.2.: HC-Emission bei cVI im Bilanzzeitraum von 40 s während des Katalysatorheizens in Abhängigkeit von $\lambda$ und Injektorspray

hier nicht dargestellt sind, ein sehr ähnliches Bild ergibt, liegt der Schluss nahe, dass mangelnde Gemischhomogenisierung und Flammenauslöschung in der Nähe von kalten Brennraumwänden („flame-quenching“) die Hauptursachen für die HC-Emissionen sind. Ein geringfügiger, globaler Sauerstoffüberschuss mit $\lambda=1,05$ kann der Entstehung von CO- und HC-Emissionen entgegenwirken. Für dieses $\lambda$ werden $N O_{x}$-Emission im Bereich von $13 \%$ des EU6-Grenzwerts gemessen. Außerdem bleibt festzuhalten, dass der Einfluss des Luftverhältnisses auf die HC-, CO- und $\mathrm{NO}_{x}$-Emissionen den Einfluss des Spray-Layouts deutlich überlagert. Dies ist nachvollziehbar, da die intensive Rückströmung auch bei ungünstigem Spraytargeting eine hinreichende Gemischbildung ermöglicht. 


\section{A.2. Veröffentlichte Erfindungsmeldung}

\begin{tabular}{|l|l|l|}
\hline BOSCH & EM-Nr. 2017/2449 & Bl. $1 / 7$ \\
\hline
\end{tabular}

Öffentlicher Aushang

BOSCH-Fe, Bregenzer Str. / Ecke Wernerstr.

von:

bis:

$26.10 .-2 \cdot 11.18$

PRS-Fe

2 C/IPE4

0

Stichwortartige Bezeichnung:

Nutzung einer Rückströmnocke zur Verbesserung der PFI-Gemischbildung

- siehe Anlage -

Abbildung A.3.: Deckblatt der Erfindungsmeldung 


\section{Nutzung einer Rückströmnocke zur Verbesserung der PFI-Gemischbildung}

\section{Stand der Technik}

Die Partikelentstehung bei der ottomotorischen Verbrennung wird maßgeblich durch Kraftstoffwandfilme im Brennraum bestimmt. Verdunsten im Brennraum vorhandene Kraftstoffwandfilme noch nach der Zündung, führt dies zur Verbrennung unter Sauerstoffmangel (Diffusionsflammen). Dabei entstehen Rußpartikel. Bei PFI-Motoren wird die Gemischbildung durch die Luftströmung am Einlassventilspalt bestimmt, und weniger durch die Güte des Primärsprays des Injektors. Bei Teillast unterhalb der saugmotorischen Volllast tritt eine Rückströmung von heißem Brennraumgas über die Einlassventile in den Einlassbereich auf. Diese rührt von einer kurzzeitigen Druckdifferenz zwischen Brennraumdruck und Saugrohrdruck. Dadurch wird der in Einlassventilnähe abgelagerte Kraftstoffwandfilm zurückgedrängt, zusätzlich aufbereitet und besser verdunstet, so dass Wandfilm im Brennraum auch nach erneutem Kraftstoffeintritt deutlich reduziert werden kann. Bei hohen Lasten und im aufgeladenen Betrieb steht diese Rückströmung nicht mehr zur Verfügung. Dadurch steigt die PN-Emission stark an.

\section{Aufgabe der Erfindung}

Der Effekt der Rückströmung soll mit der Erfindung auch bei hohen Lasten zur Verfügung stehen. Damit wird in allen Betriebspunkten die Gemischaufbereitung des Kraftstoffes in Ventilspaltnähe verbessert. Der Kraftstoff wird dadurch größtenteils gasförmig in den Brennraum eingebracht und Wandfilme werden vermieden. Die PNEmissionen werden bei diesen bislang kritischen Betriebspunkten stark reduziert.

\section{Kern und Vorteil der Erfindung, besonders gegenüber dem Bekannten}

Während der Kompressions- oder Expansionsphase liegt im Brennraum ein höherer Druck an als im Saugrohr. Können zu diesen Zeitpunkten die Einlassventile mit einem kleinen, zusätzlichen Hub geöffnet werden, ist eine Rückströmung zur besseren Gemischaufbereitung gegeben. Der Ventilhub wird üblicherweise über eine Nockenwelle gesteuert. Mittels eines kurzen Zusatzhubs auf der Einlassnocke soll dieser zusätzliche Ventilhub erzeugt werden. Durch die Kürze der Rückströmung werden die Vollastfüllung und die Restgasrate nur geringfügig beeinträchtigt. Während bislang bei Motorbetriebspunkten mit hoher Last, insbesondere im aufgeladenen Kennfeldbereich, 
Kraftstoffwandfilme im Brennraum in der Nähe der Einlassventile zu erheblichen PNEmissionen führen, kann die Rückströmung durch die Zusatznocke diese Wandfilme und resultierende PN-Emissionen reduzieren.

\section{Detaillierte Beschreibung von Aufbau und Funktion des Vorschlags}

Der zusätzliche Ventilhub wird durch eine weitere Erhöhung, zusätzlich zum Hauptöffnungshub, auf dem Umfang jeder Einlassnocke angebracht. Dieser zusätzliche Ventilhub muss sehr klein sein $(<0,5 \mathrm{~mm})$ und auch entsprechend kurz $\left(\approx 30^{\circ} \mathrm{KW}=\right.$ $15^{\circ} \mathrm{NW}$ ), damit die einhergehenden Füllungsverluste im Kompressionstakt bzw. die Erhöhung der Restgasrate im Expansionstakt klein bleiben. Aus thermodynamischer Perspektive bieten sich zwei Lösungsmöglichkeiten:

\section{Zweitnocke im Kompressionstakt}

Bei diesem Lösungsvorschlag wird der im Vergleich zum Saugrohr höhere Druck im Brennraum zu Beginn der Kompressionsphase vor der Verbrennung ausgenutzt. Die Gestalt der Nockenkontur mit Haupt- und Zusatzventilhub ist in Abbildung A.4a schematisch skizziert. Es ist ebenfalls denkbar, dass die Zusatznocke im Kompressionstakt als eine Verlängerung der Einlassnocke mit sehr geringem Hub ausgeführt wird.

\section{Zusatz-Nocke Kompression}

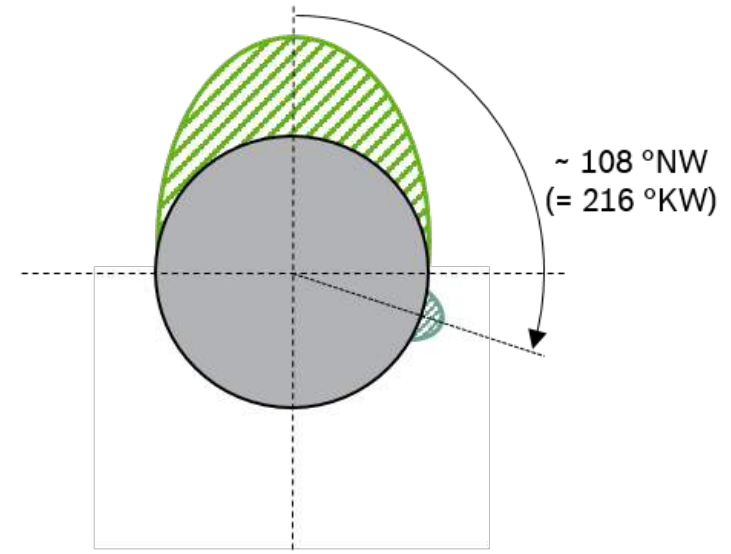

(a) Zusatznocke im Kompressionstakt

\section{Zusatz-Nocke Expansion}

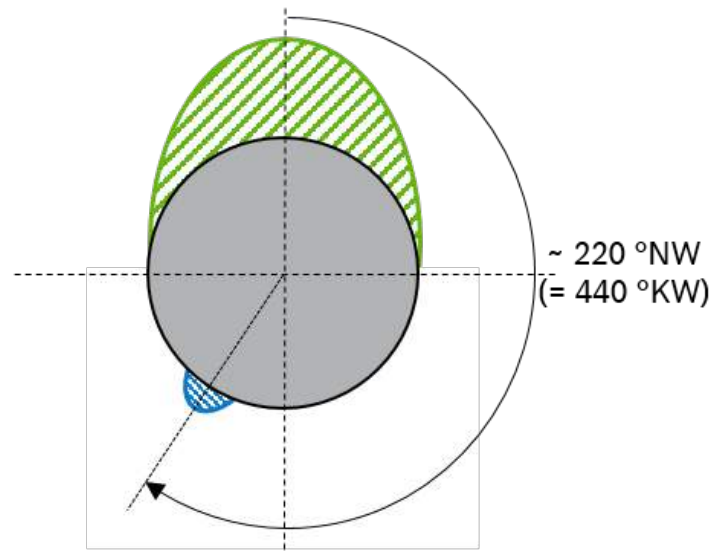

(b) Zusatznocke im Expansionsstakt

Abbildung A.4.: Zusatznocken mit beispielhaftem Öffnungszeitpunkt 


\section{Zweitnocke im Expansionstakt}

Bei diesem Lösungsvorschlag wird der im Vergleich zum Saugrohr höhere Druck im Brennraum gegen Ende der Expansionsphase nach der Verbrennung ausgenutzt. Die Gestalt der Nockenkontur mit Haupt- und Zusatzventilhub ist in Abbildung A.4b schematisch skizziert.

\section{Weitere Erläuterungen \& Diskussion}

Beispielhafte Öffnungszeitpunkte und Erhebungskurven für die Zusatzventilhübe durch die Zusatznocken sind in Abbildung A.5 für die beiden Lösungsvorschläge „Zusatznocke im Kompressionstakt“ und „Zusatznocke im Expansionstakt“ skizziert. Die kon-

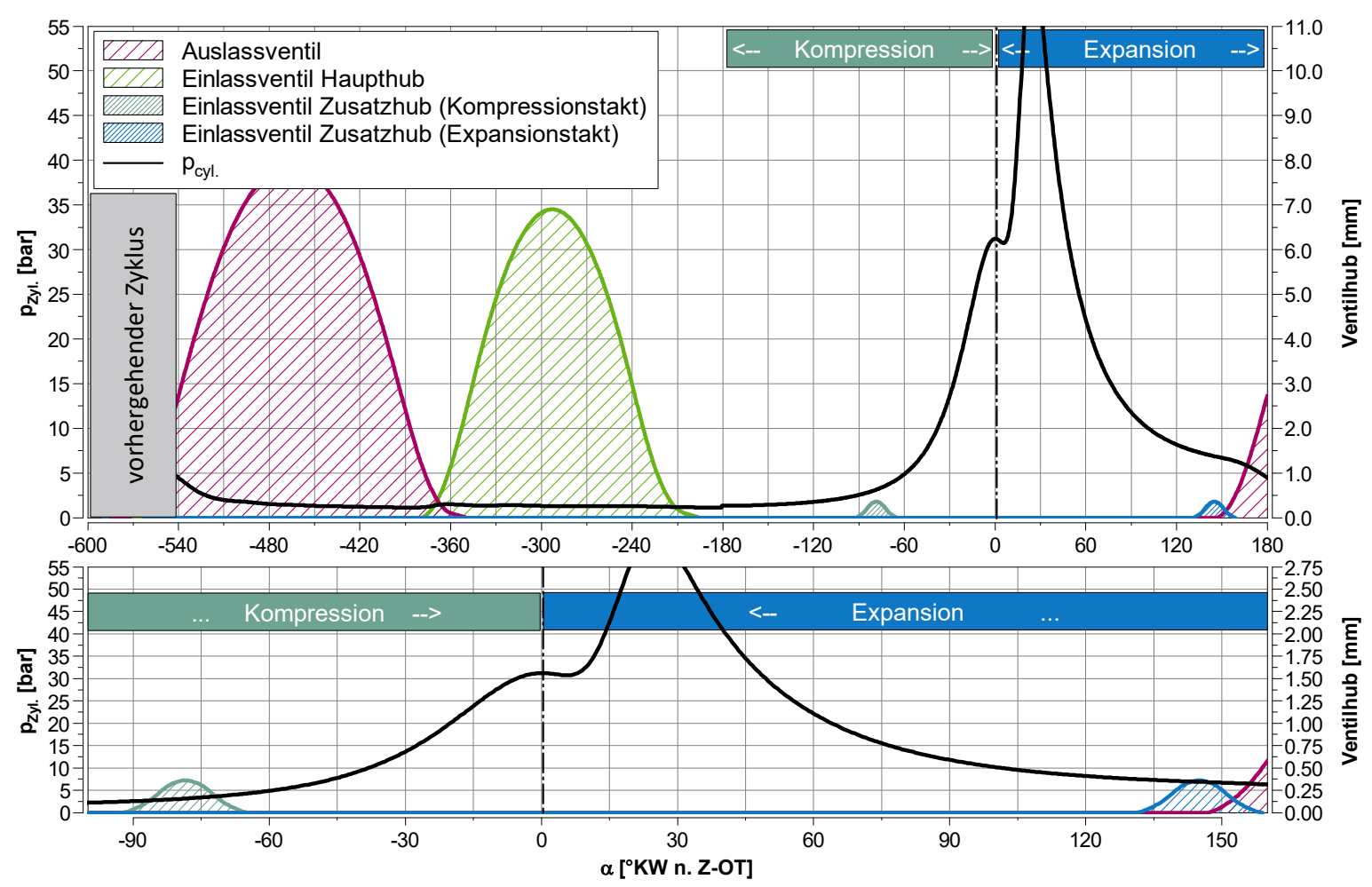

Abbildung A.5.: Beispielhafte Öffnungszeitpunkte und Ventilerhebungskurven für Lösung 1 und 2

kreten Werte für Hub und Länge des Zusatzhubes müssen für jedes Motorprojekt individuell berechnet werden. Dabei gilt es beim Zusatzhub in der Kompressionsphase den Kompromiss zwischen Intensität der Rückströmung und Füllungsverlust einzugehen. Bei kleinen Zusatzhüben mit kurzer Länge bleibt der Füllungsverlust klein, jedoch ist auch die Rückströmung wenig ausgeprägt. Bei großen Zusatzhüben und längerer Öffnungsdauer ist die Rückströmung wirksamer, jedoch steigt auch der Verlust an Zylinderfüllung. Beim Zusatzhub in der Expansionsphase gilt es den Kompromiss zwischen Intensität der Rückströmung und Erhöhung der Restgasrate zu finden. 
Der Zusatzventilhub in der Expansionsphase ermöglicht das Rückströmen von heißen Verbrennungsgasen in den Einlasskanal. Neben der gewünschten Aufbereitung der Kraftstoffwandfilme mischt sich dieses Abgas auch mit der frischen Ladung und führt somit zu einer Steigerung der internen Abgas-Rückführung (AGR). Die somit erhöhte Restgas-Rate des Luft-Kraftstoffgemischs darf nicht beliebig gesteigert werden, da die Klopfneigung steigen kann und die Stabilität der Verbrennungen beeinträchtigt wird (Laufruhe).

Alternativ zur bisher diskutierten Möglichkeit, bei der der Zusatzhub immer wirksam ist, könnte auch eine zusätzliche Nocke angebracht werden. Diese zusätzliche Nocke ist mit Haupt- und Zusatzhub versehen und ist direkt neben der "Standard-Nocke“ ohne Zusatzhub platziert. Die Nocke mit Zusatz-Hub wäre dann eine wählbare Option, wenn die Aktivierung der einen oder anderen Nocke beispielsweise über eine Verschiebung der Nocken auf der Nockenwelle ausgeführt ist Abbildung A.5). Solche Schiebe-Nocken-Lösungen sind bereits für Ventilhubverstellung in Serie. Somit könnte bei Bedarf auch mit der „Standard-Nocke“ ohne Zusatz-Hub gefahren werden, und Füllungsverluste damit vermieden werden.

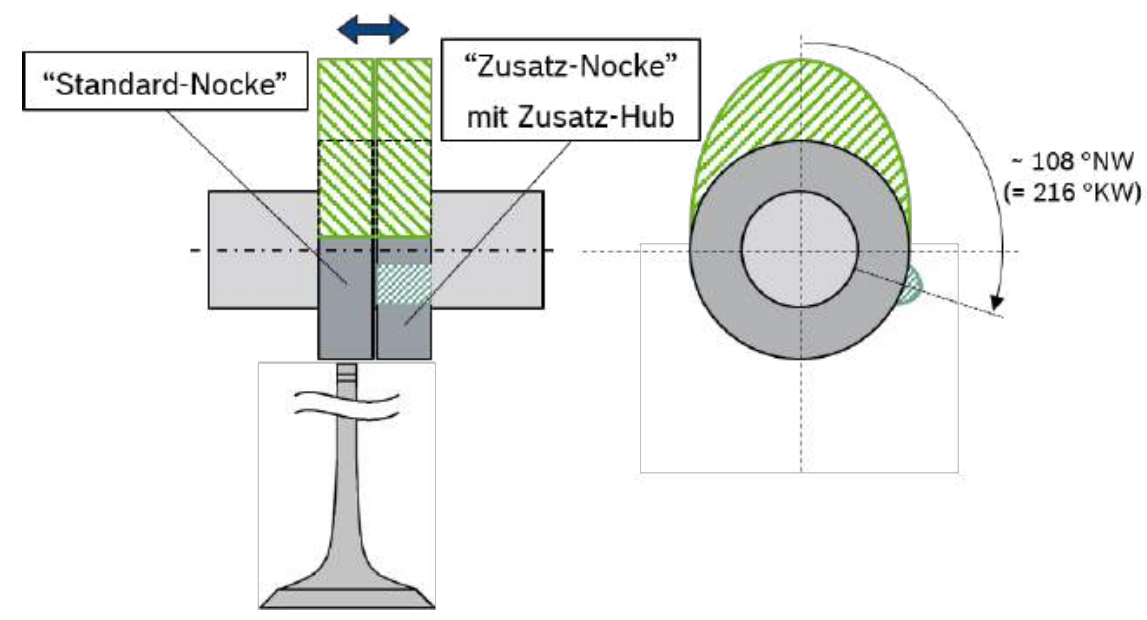

Abbildung A.6.: Zusatz-Hub auf zweiter Nocke zur Wahl zwischen Standard-Nocke und „Zusatz-Hub-Nocke“ durch Verschiebung der Nocken auf der Nockenwelle 


\section{A.3. Ergänzendes Informationsmaterial}

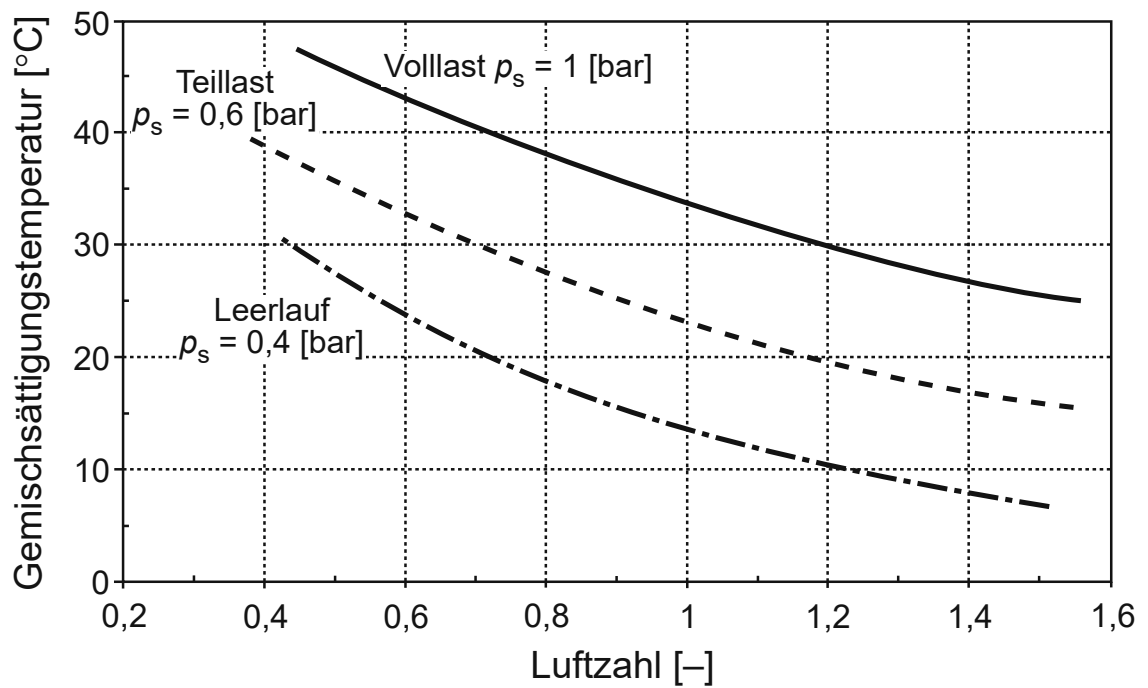

Abbildung A.7.: Gemischsättigungstemperaturen für Superkraftstoff [30, 107] 


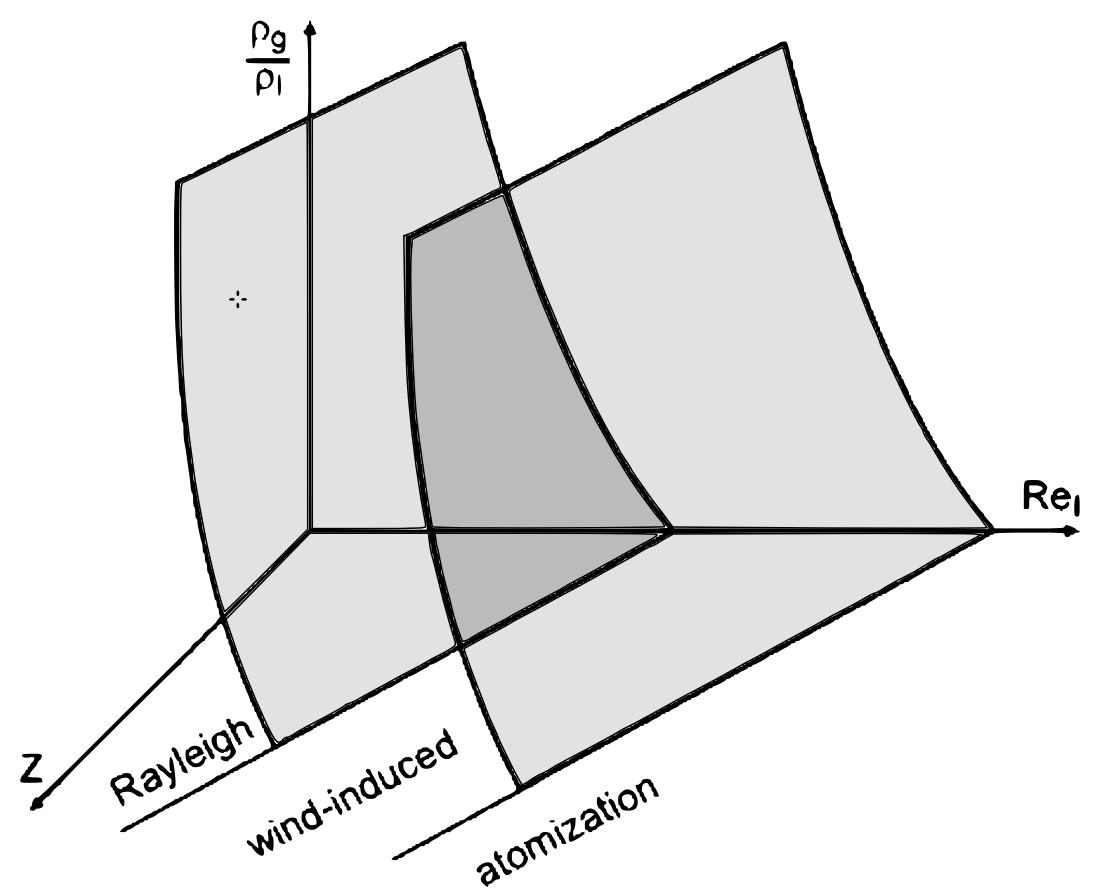

Abbildung A.8.: Einfluss der Gasdichte auf den Strahlzerfall (Primärzerfall) nach Reitz [90].

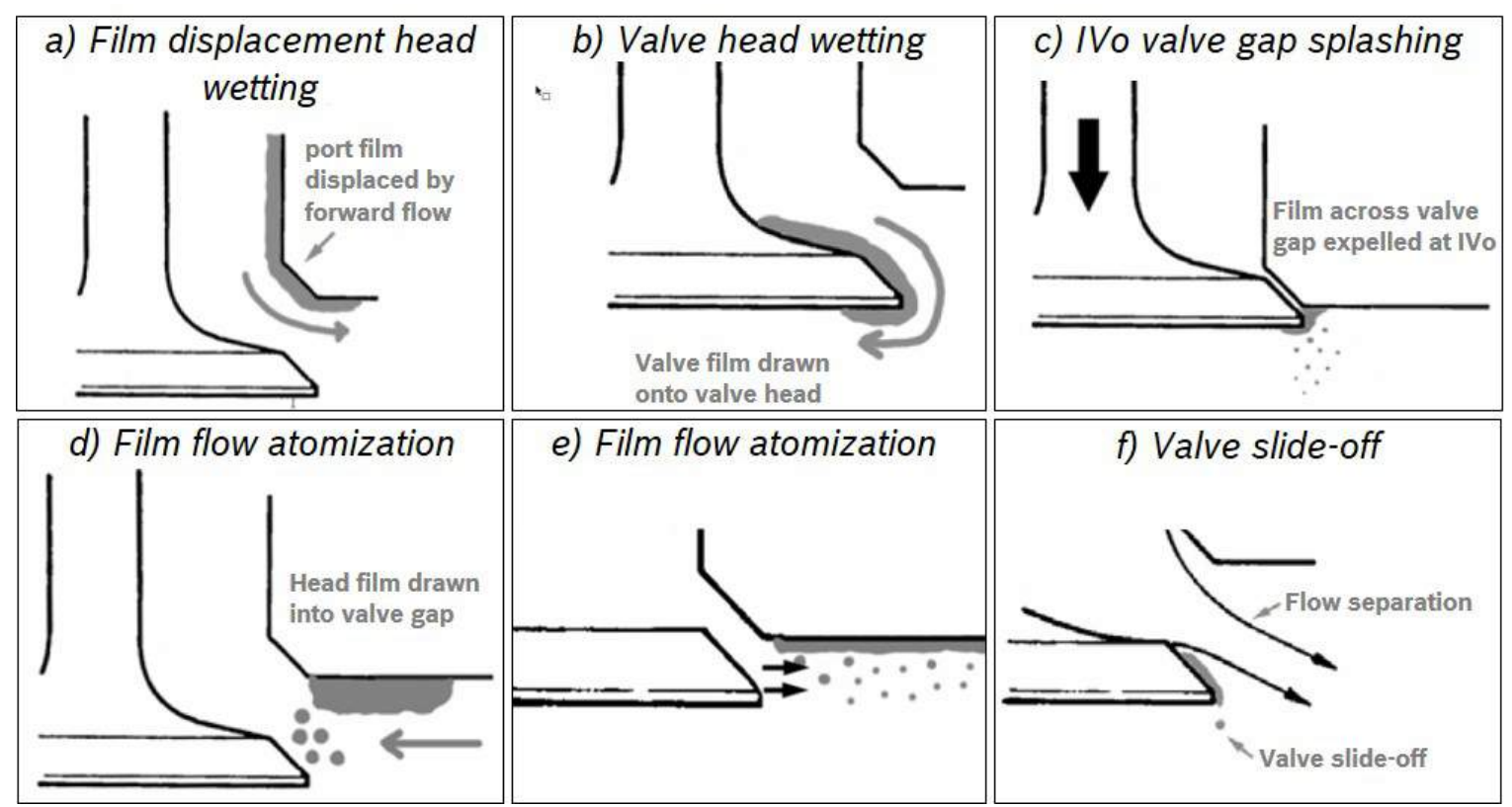

Abbildung A.9.: Wandfilmtransportvorgänge nach Costanzo u. Heywood [16] für niedrige Gasgeschwindigkeit a) - c) und für hohe Gasgeschwindigkeit d) - f) 


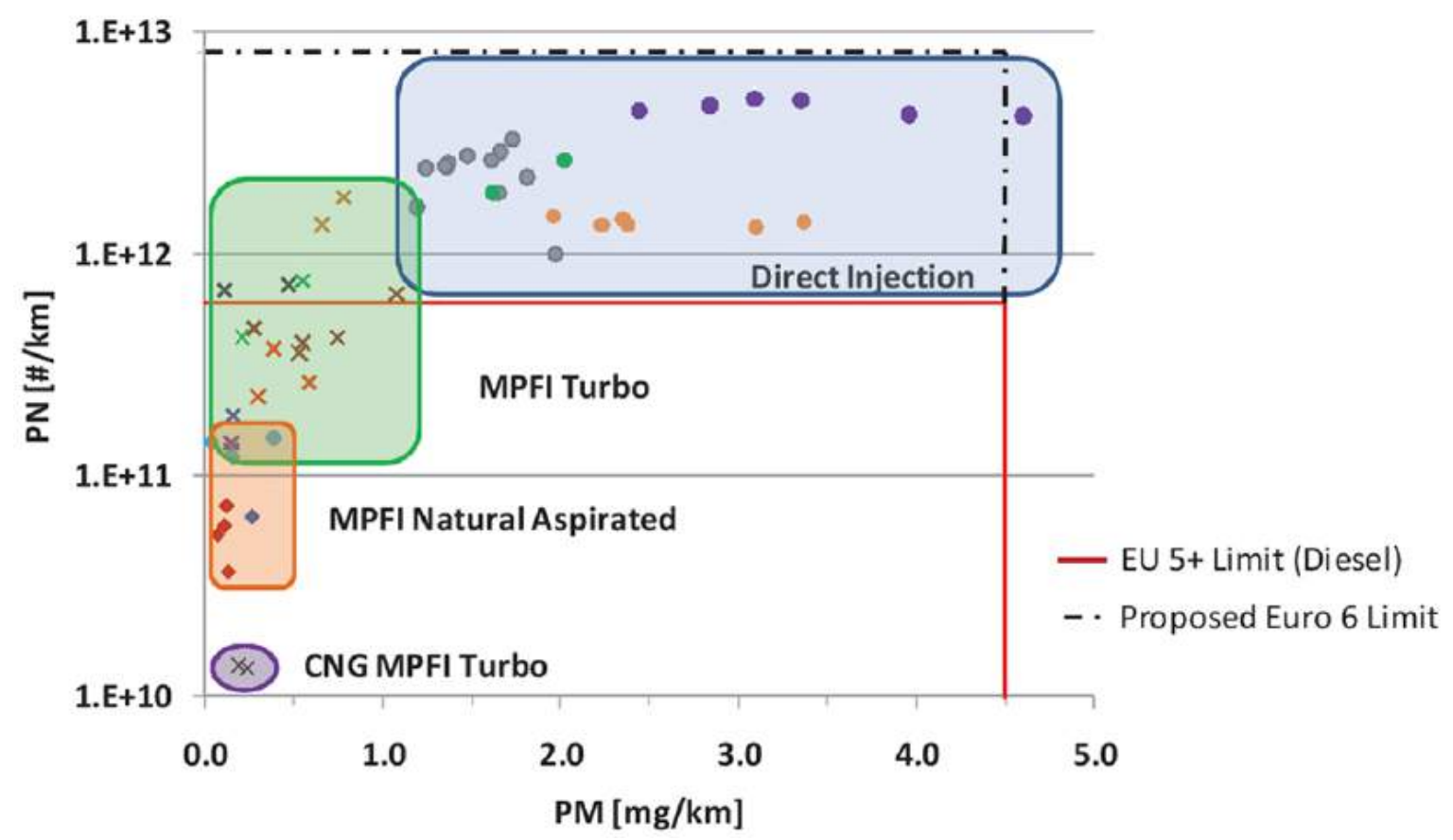

Abbildung A.10.: Klassierung von PN- und PM-Emissionen aus Fahrzeugmessungen nach Sabathil [98] (Zwischenzeitlich ist der EU6 Grenzwert für die Partikelanzahlemissionen mit $6 \cdot 10^{11} \# / \mathrm{km}$ bekannt und in Kraft)

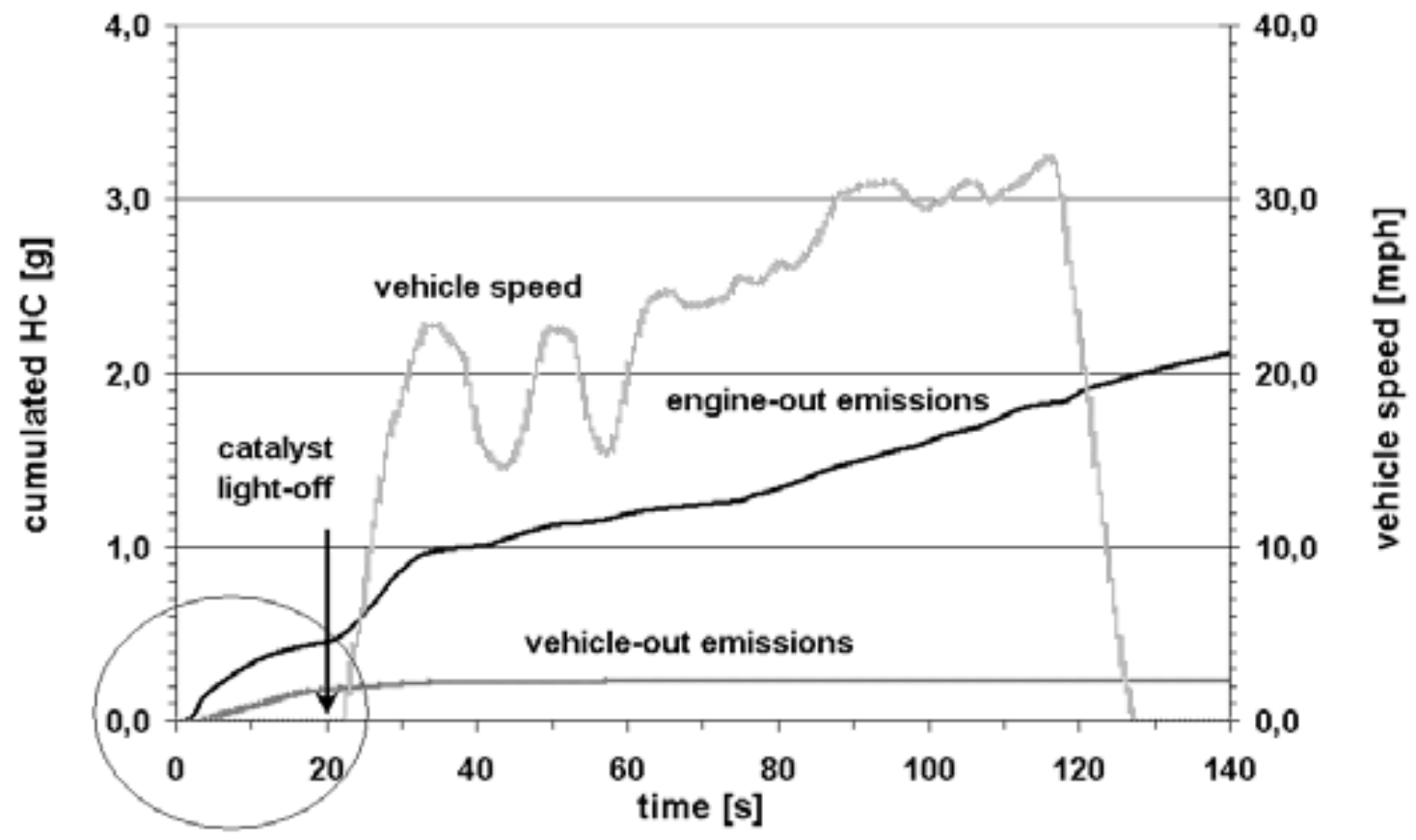

Abbildung A.11.: Auszug der HC-Emissionen eines Fahrzeugs mit SRE während eines FTP75-Fahrzyklus 

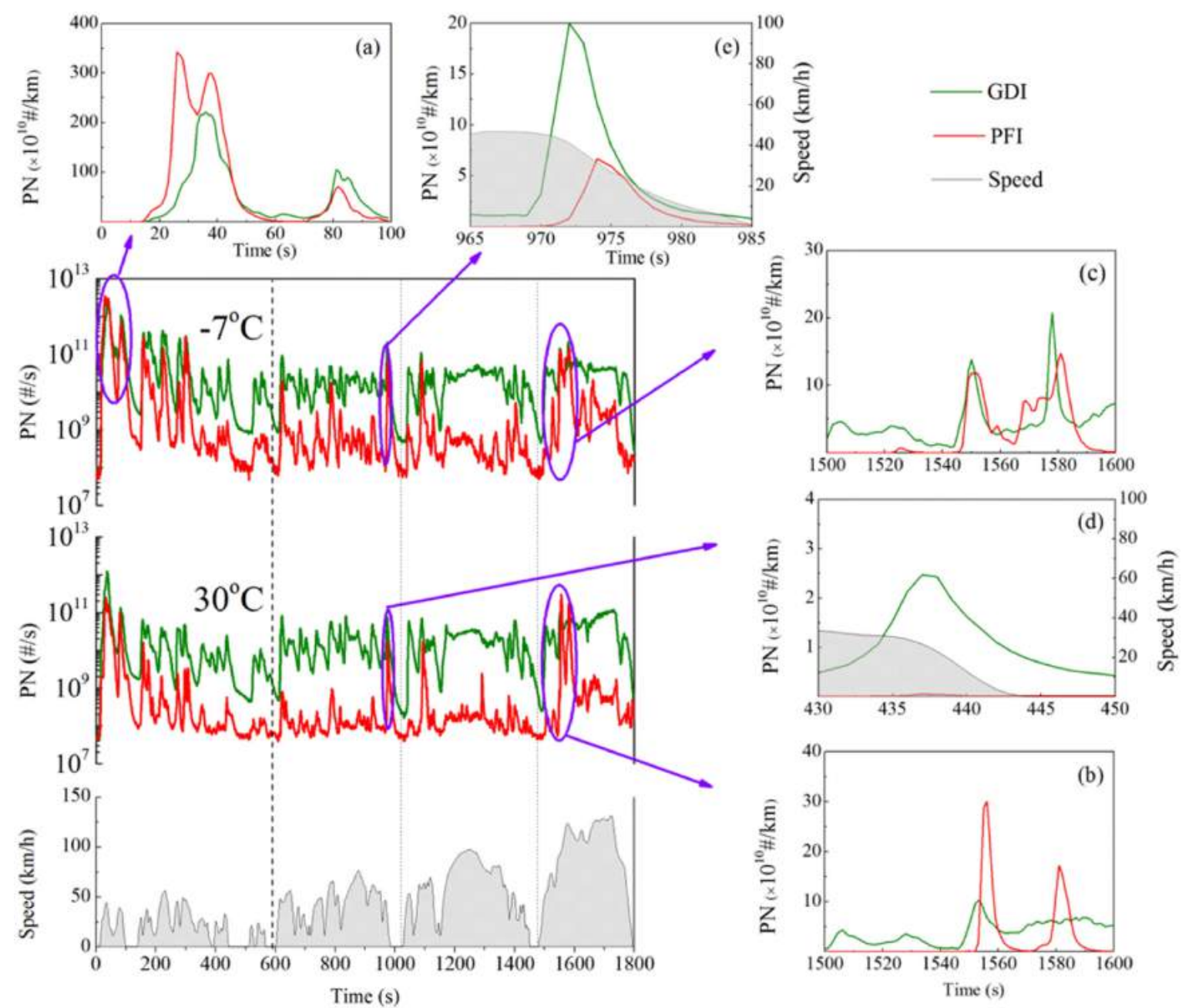

Abbildung A.12.: Zeitbasierte Daten von WLTC Fahrzyklusmessungen eines SRE-Fahrzeugs und eines BDE-Fahrzeugs bei verschiedenen Starttemperaturen $\left(30^{\circ} \mathrm{C}\right.$ und $\left.-7^{\circ} \mathrm{C}\right)$ 

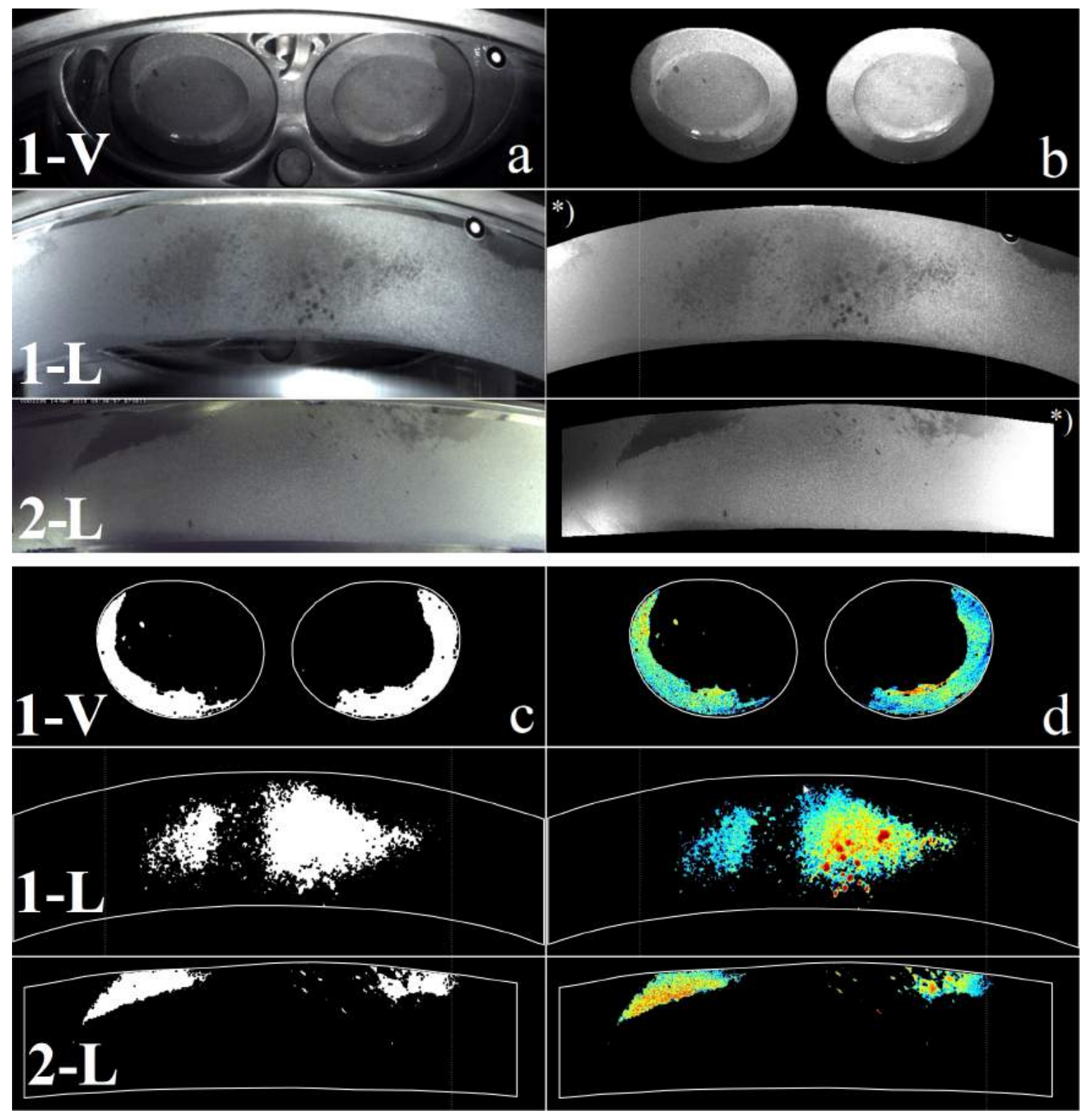

Abbildung A.13.: Vergrößerte Ansicht der Beispiele zur Methodik der Wandfilmquantifizierung am Transparentmotor für alle drei Perspektiven (1-V, 1-L, 2-L) in vier Schritten (a,b,c,d), siehe Kapitel 3.2.2 


\begin{tabular}{|c|c|c|c|c|c|}
\hline Injektor & $\mathbf{A}$ & B & $\mathbf{C}$ & D & $\mathbf{E}$ \\
\hline Konfiguration & Single & Single & Single & Single & Single \\
\hline Düsentyp & $\mathrm{E}$ & $\mathrm{E}$ & $\mathrm{E}$ & $\mathrm{E}$ & $\mathrm{E}$ \\
\hline$\alpha \quad\left[{ }^{\circ}\right]$ & 20,4 & 22,4 & 27,0 & 33,3 & 27,4 \\
\hline$\beta \quad\left[{ }^{\circ}\right]$ & 8,8 & 11,3 & 10,4 & 10,4 & 13,8 \\
\hline$\gamma \quad\left[{ }^{\circ}\right]$ & 11,4 & 7,4 & 4,8 & 4,9 & 3,5 \\
\hline $\mathbf{Q}_{\text {stat }}[\mathrm{g} / \mathrm{min}]$ & 106,0 & 106,0 & 106,0 & 106,0 & 106,0 \\
\hline SMD $[\mu \mathrm{m}]$ & 85,7 & 70,0 & 67,8 & 68,1 & 55,9 \\
\hline Seitenansicht & & & & & \\
\hline Draufsicht & & & & & \\
\hline
\end{tabular}

\begin{tabular}{|c|c|c|c|c|}
\hline Injektor & $\mathbf{F}$ & $G$ & $\mathbf{H}$ & I \\
\hline Konfiguration & Single & Single & Single & Single \\
\hline Düsentyp & $\mathrm{E}$ & $\mathrm{E}$ & $\mathrm{E}$ & $\mathrm{C}$ \\
\hline $\boldsymbol{\alpha} \quad\left[{ }^{\circ}\right]$ & 35,0 & 36,0 & 39,5 & 28,0 \\
\hline $\boldsymbol{\beta} \quad\left[{ }^{\circ}\right]$ & 25,0 & 30,0 & 23,3 & - \\
\hline$\gamma \quad\left[{ }^{\circ}\right]$ & 0,0 & 0,0 & 0,0 & 0,0 \\
\hline$Q_{\text {stat }}[\mathrm{g} / \mathrm{min}]$ & 116,0 & 110,0 & 122,0 & 75,0 \\
\hline SMD $[\mu \mathrm{m}]$ & 57,9 & 49,6 & 52,9 & 40,0 \\
\hline Seitenansicht & & & & \\
\hline Draufsicht & & & & \\
\hline
\end{tabular}

Abbildung A.14.: Kenndaten und CAD-Visualisierungen der Spray-Auslegungen der Single-SRE-Injektoren, siehe Kapitel 3.4 

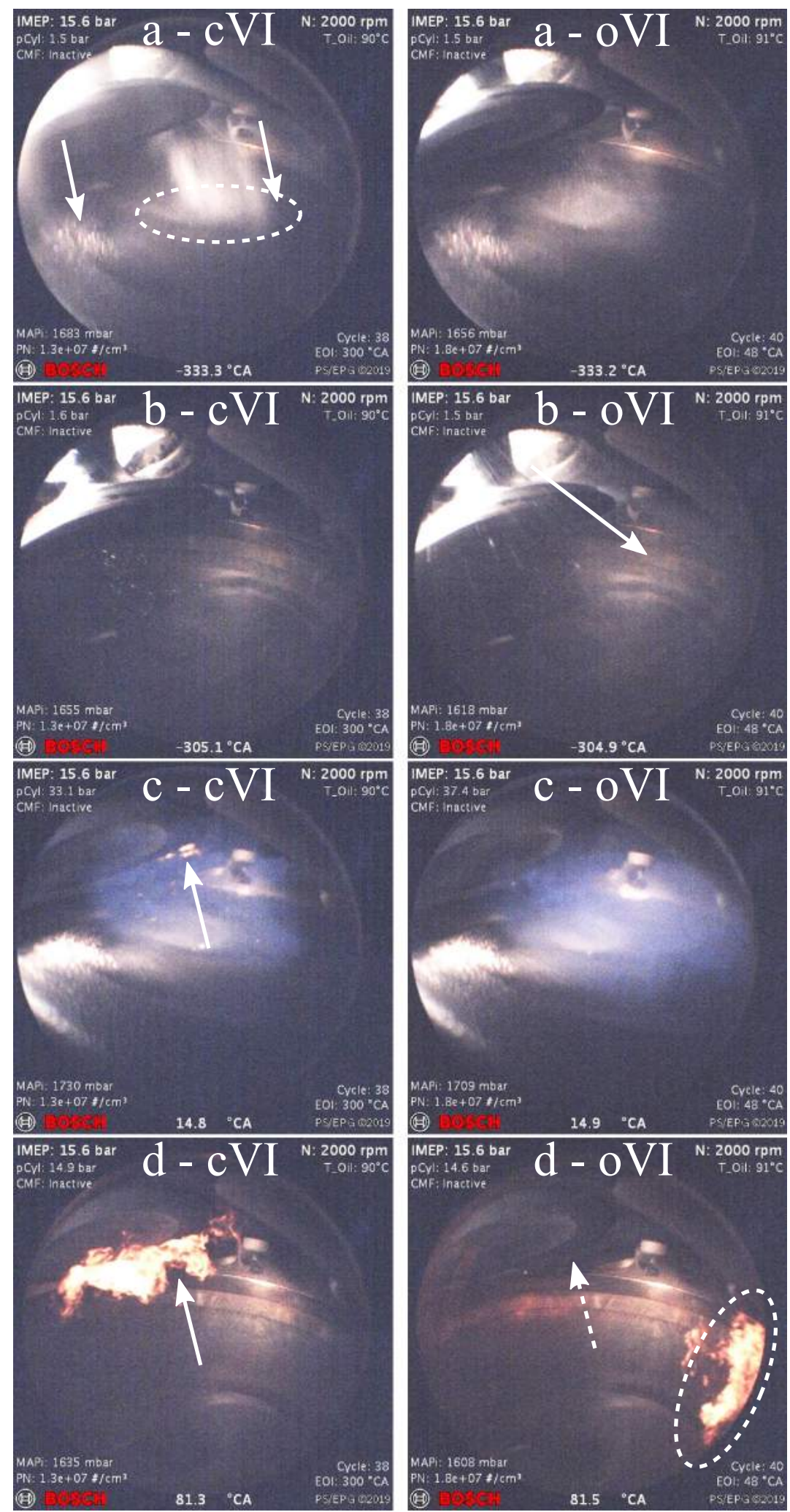

Abbildung A.15.: Visualisierung von Ansaugung und Verbrennung eines Arbeitsspiels mittels HSVE bei $N=2000 \mathrm{~min}^{-1}$, IMEP $=15,6$ bar und vorgelagerter Einspritzung mit EOI $=300^{\circ} \mathrm{KW}$ v. EVs (links) und saugsynchroner Einspritzung mit EOI $=48^{\circ} \mathrm{KW}$ v. LW-OT (rechts), siehe Kapitel 4.2 .3 


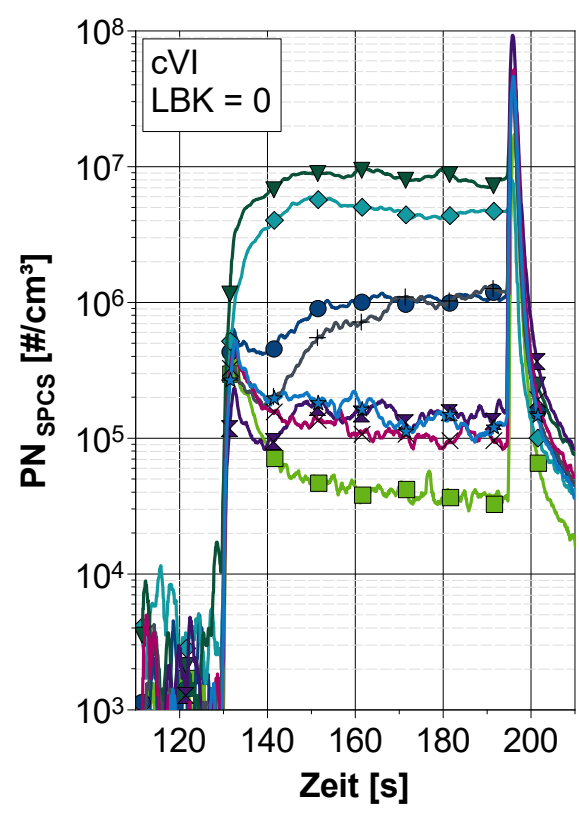

Abbildung A.16.: PN-Emissionen

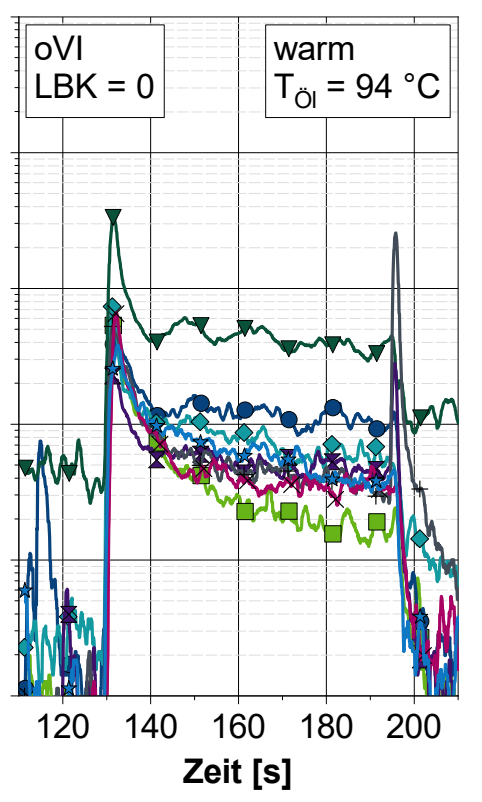
$T_{\mathrm{O} l}=94^{\circ} \mathrm{C}$ für drei Parameter-Kombinationen, siehe Kapitel 4.2 .6

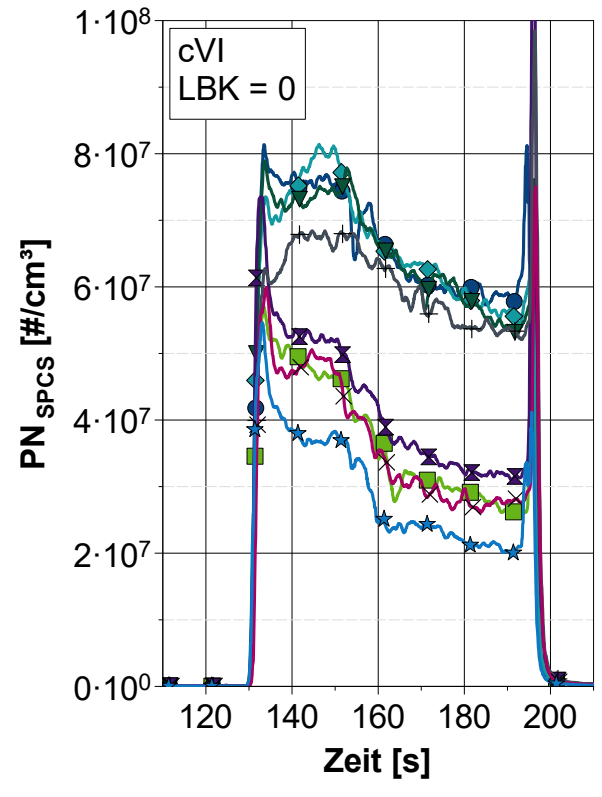

Abbildung A.17.: PN-Emissionen

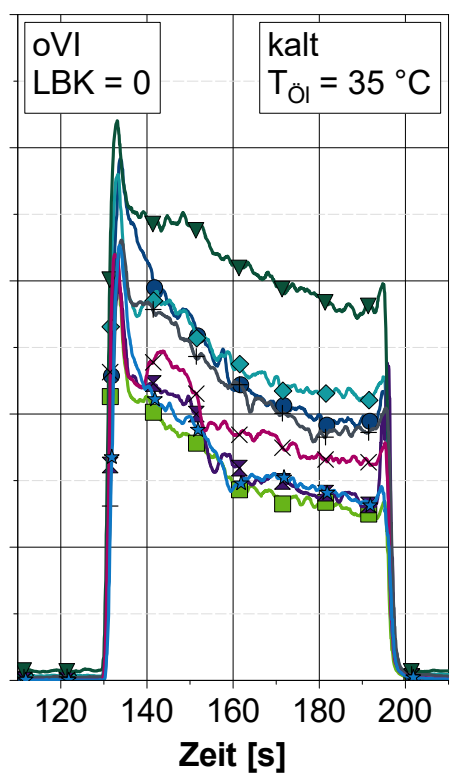

gemittelt über drei

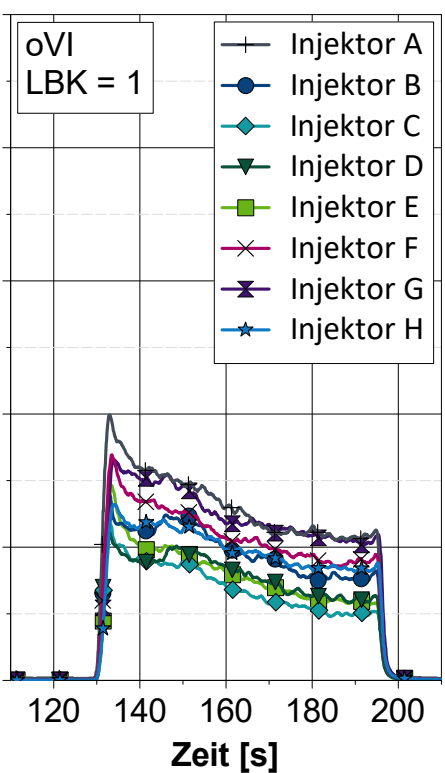
$I M E P_{1}=2$ bar $\rightarrow I M E P_{2}=12$ bar bei kaltem Motor mit $T_{\ddot{\mathrm{O}} l}=35^{\circ} \mathrm{C}$ für drei Parameter-Kombinationen, siehe Kapitel 4.2.6 

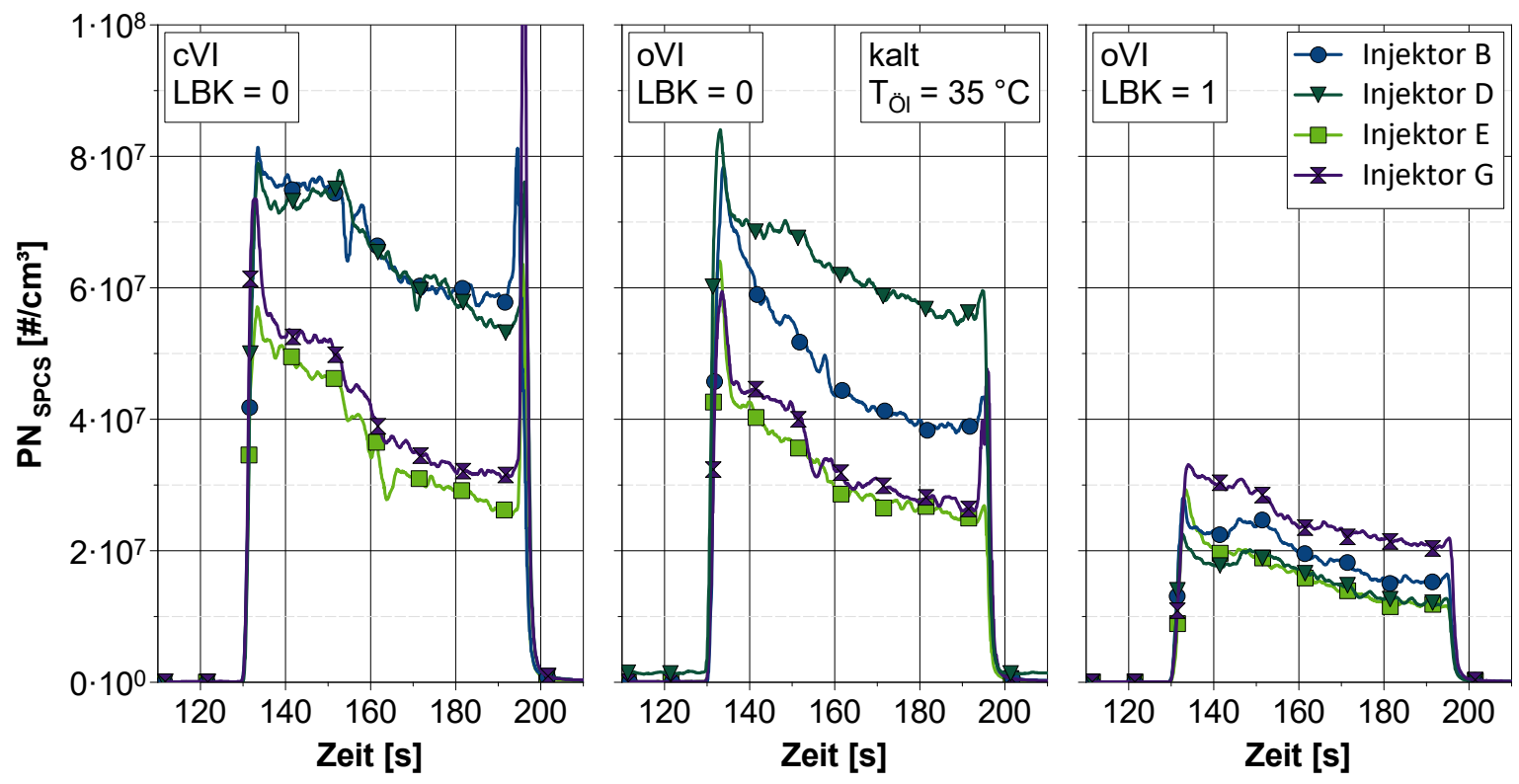

Abbildung A.18.: PN-Emissionen gemittelt über drei Lastsprünge von $I M E P_{1}=2$ bar $\rightarrow I M E P_{2}=12$ bar bei kaltem Motor mit $T_{\mathrm{Ö} l}=35^{\circ} \mathrm{C}$ für drei Parameter-Kombinationen

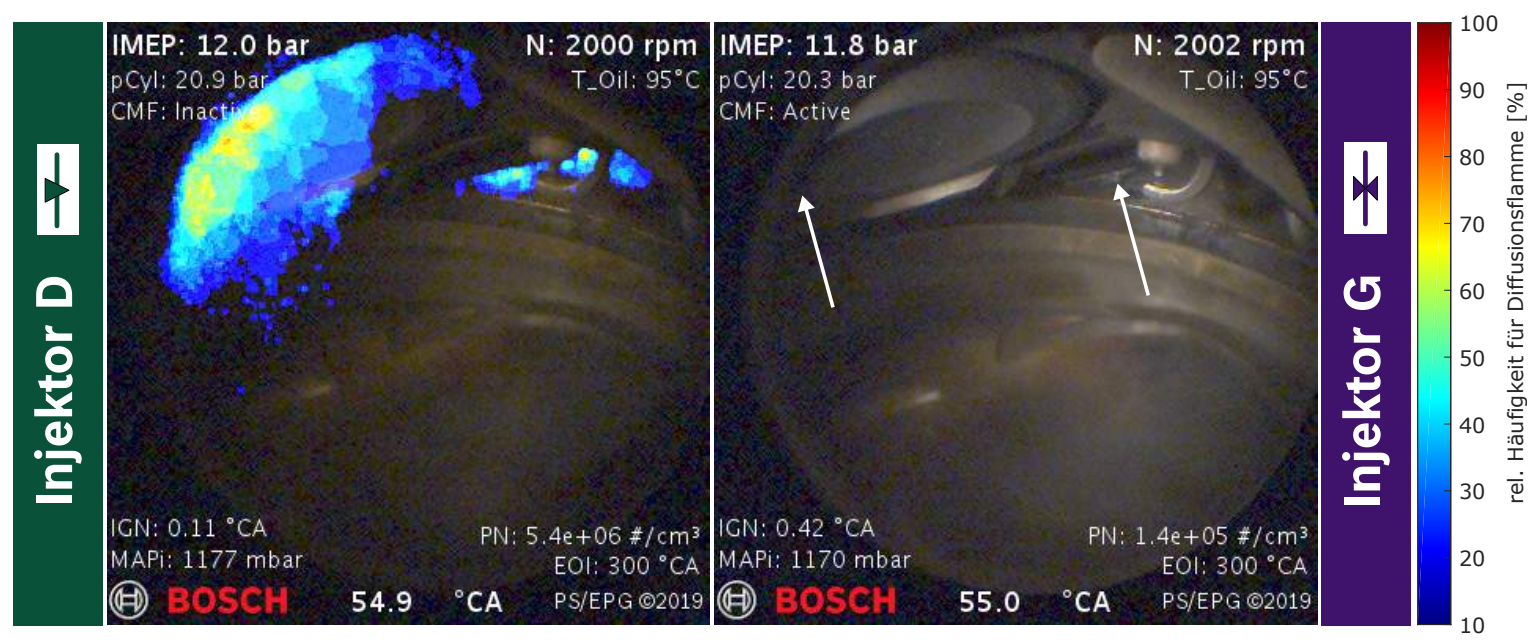

Abbildung A.19.: Gegenüberstellung der Auftretenswahrscheinlichkeit von Diffusionsflammen von Injektor $\mathrm{G}$ und $\mathrm{C}$ mittels HSVE bei $I M E P_{2}=12$ bar, $\mathrm{cVI}, \mathrm{LBK}=0$ und warmem Motor, siehe Kapitel 4.2 .6 

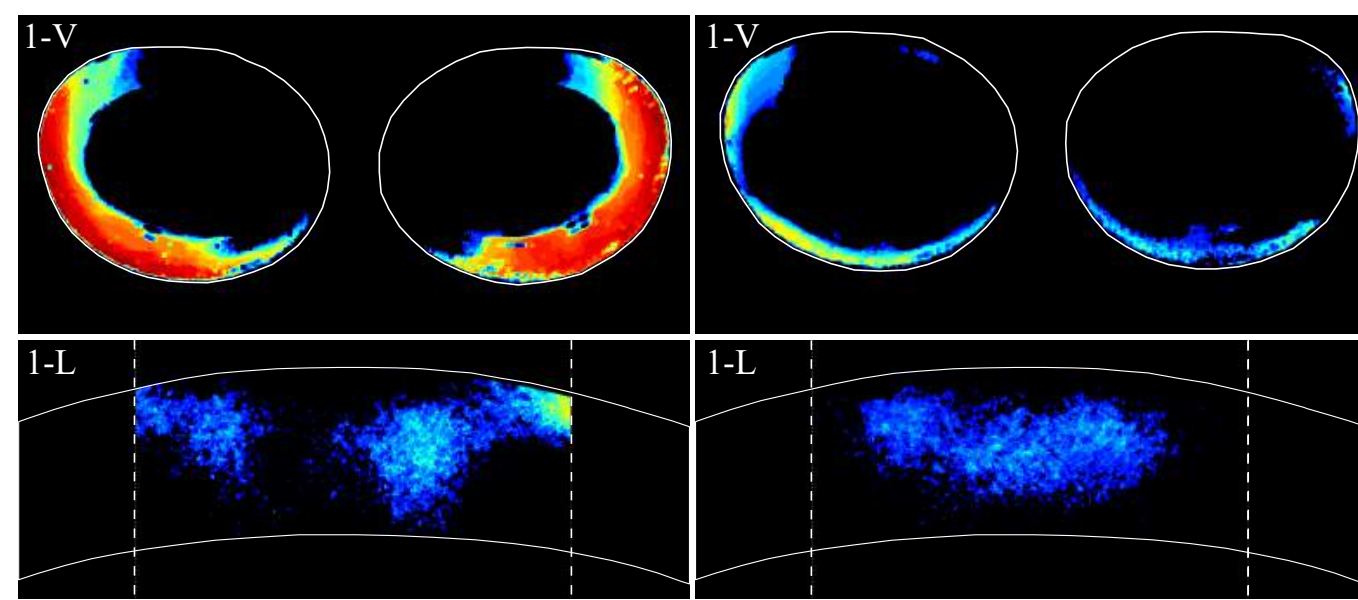

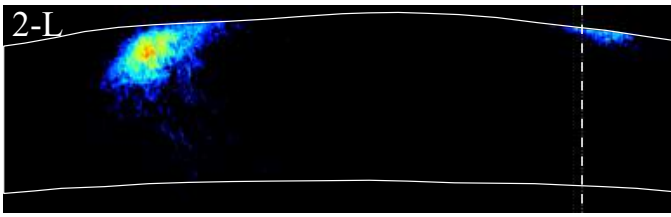

Injektor D | cVI | LBK0

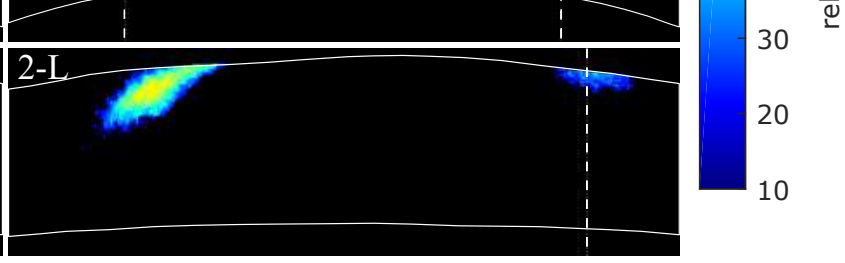

Injektor $\mathrm{G}|\mathrm{cVI}| \mathrm{LBK} 0$

Abbildung A.20.: Gegenüberstellung der Auftretenswahrscheinlichkeit von Wandfilm nach 30 Zyklen bei vorgelagerter Einspritzung (cVI) und $L B K=0$ mit Injektor D (links) und Injektor G (rechts), siehe Kapitel 4.2.6

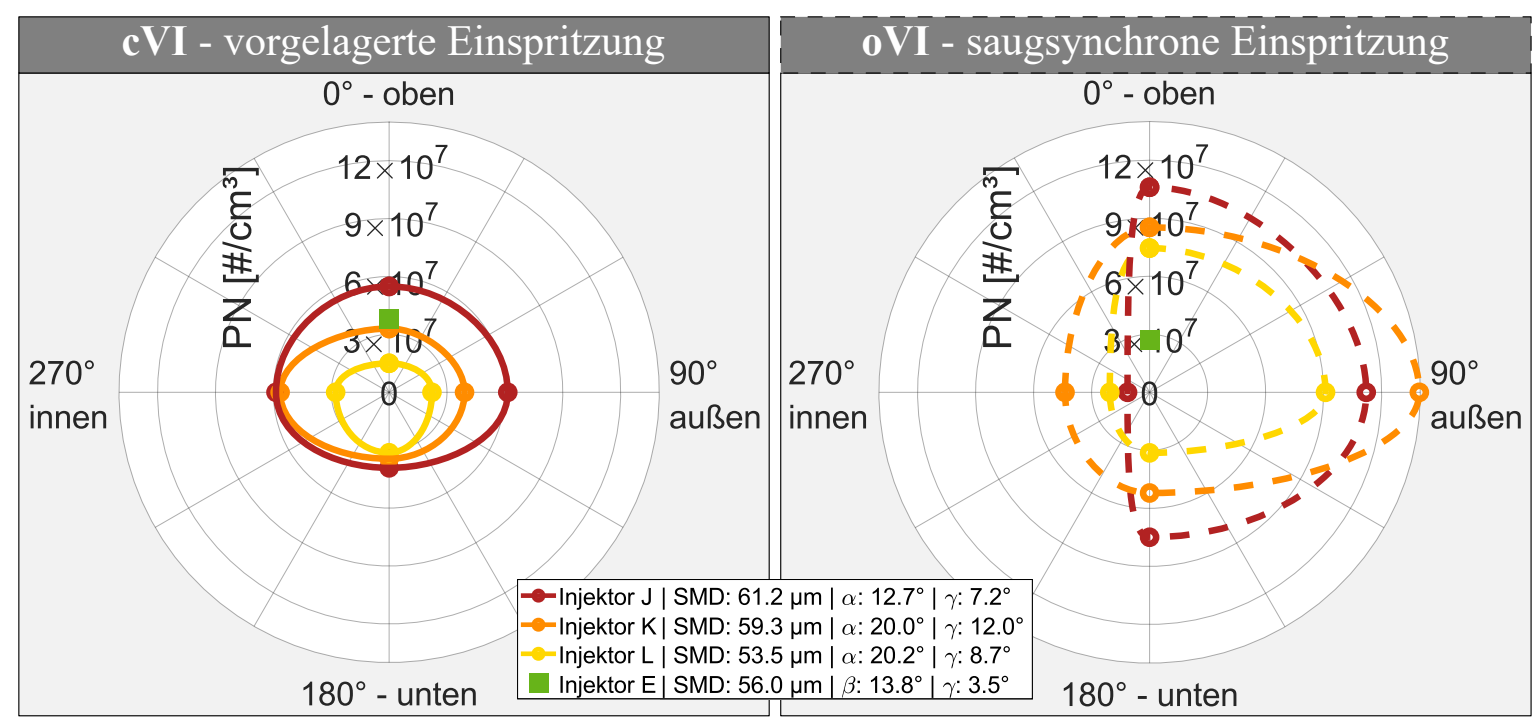

Abbildung A.21.: PN-Emissionen in Abhängigkeit des Spray-Targetings, variiert durch Rotation der Injektoren, bei IMEP $=12$ bar, LBK $=0$ und kaltem Motor, siehe Kapitel 4.2 .7 

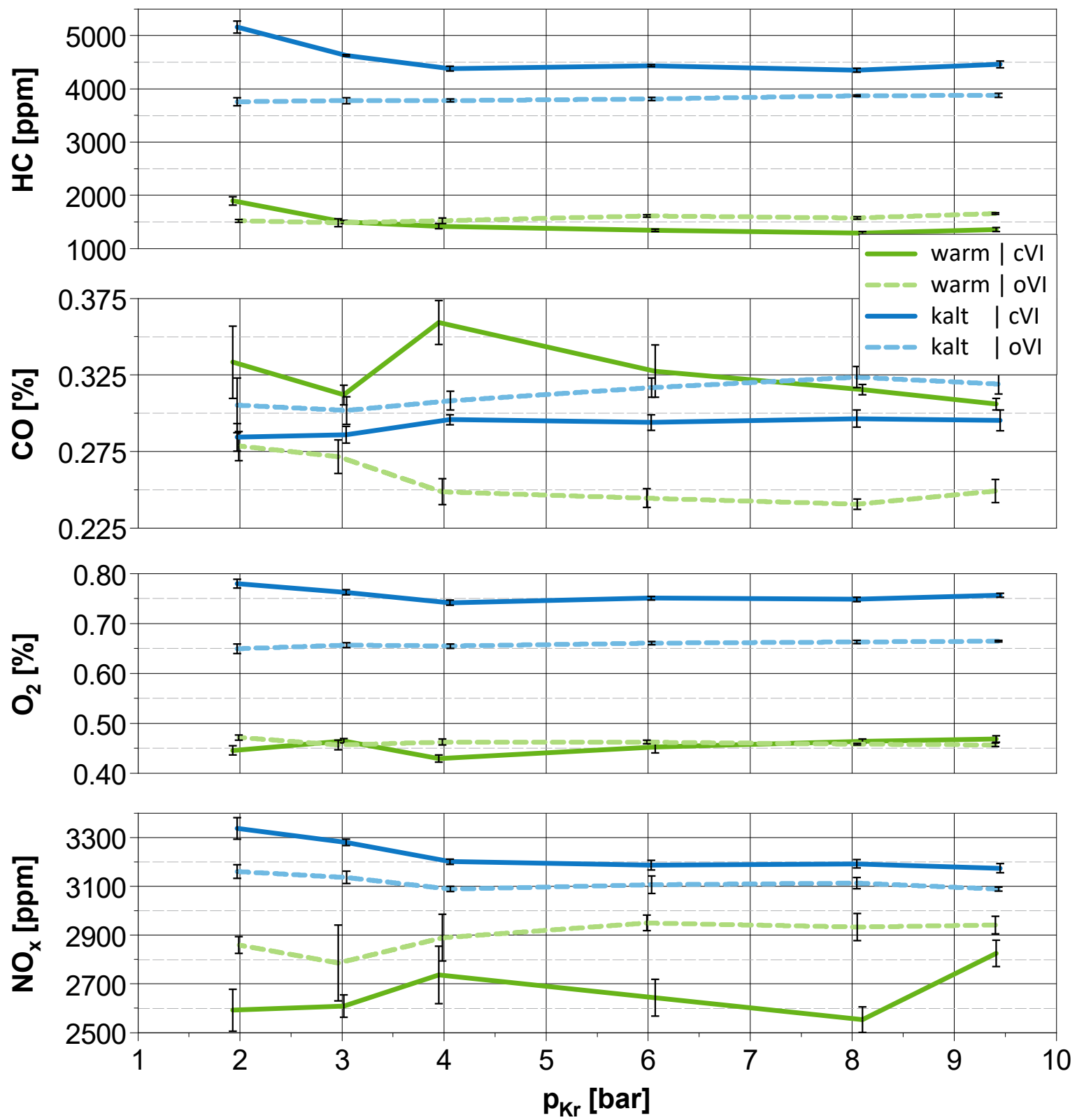

Abbildung A.22.: Gasförmige Schadstoffemissionen und Restsauerstoff bei Variation des Kraftstoffdrucks $p_{K r}$ mit Injektor E, siehe Kapitel 4.2 .8 


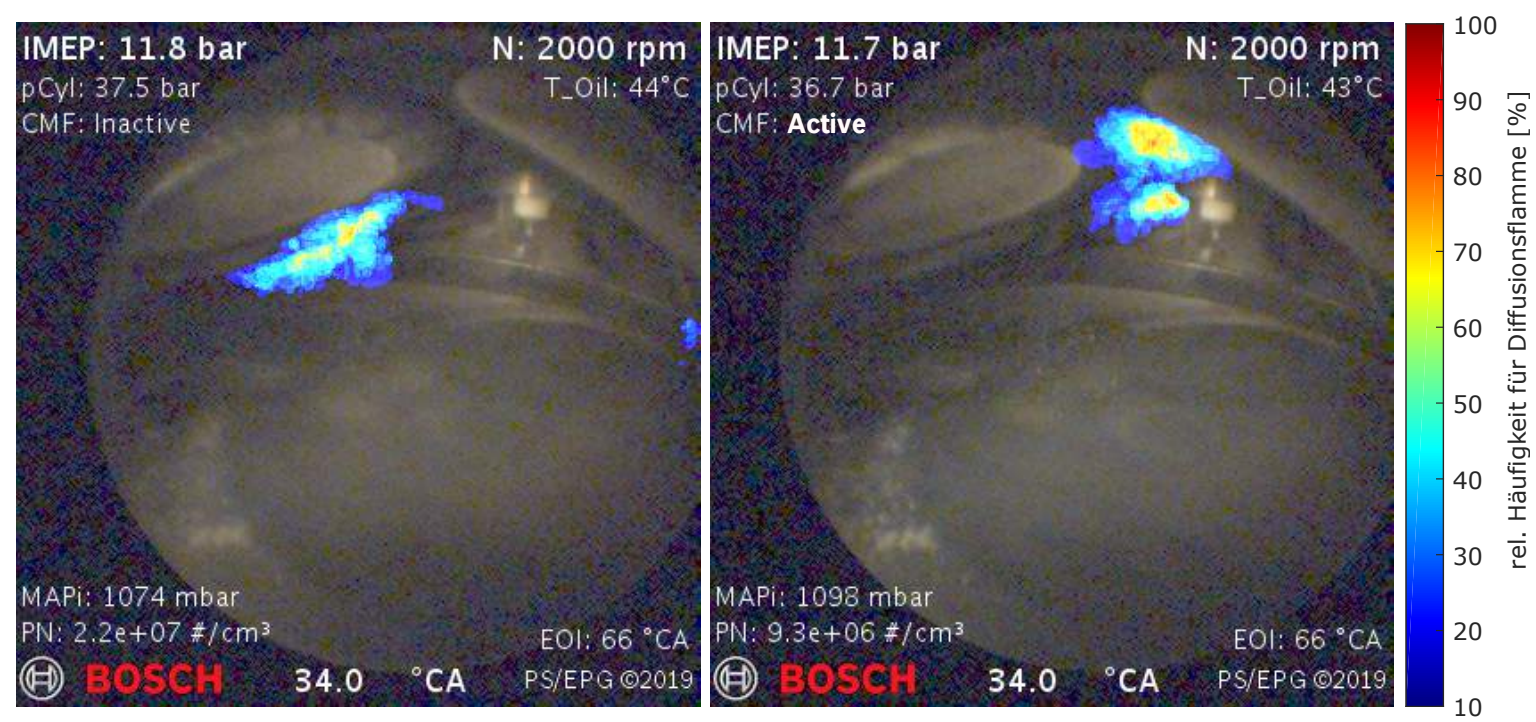

Abbildung A.23.: Gegenüberstellung der räumlichen Auftretenswahrscheinlichkeit von Diffusionsflammen bei $I M E P=12$ bar mit deaktivierter LBK (links) und aktivierter LBK (rechts) mit Single-Injektor E, siehe Kapitel 4.2 .7

\begin{tabular}{lll}
\hline & Horiba SPCS2100 & Cambustion DMS500 \\
\hline Messprinzip & $\begin{array}{l}\text { Laser scattering } \\
\text { condensation particle } \\
\text { counting }(\mathrm{CPC})\end{array}$ & Koronaentladung \\
Größenspektrum & $23-3000 \mathrm{~nm}$ & $5-1000 \mathrm{~nm}$ \\
Ansprechverhalten & $t_{95}=3000 \mathrm{~ms}$ & $t_{90}=200-300 \mathrm{~ms}$ \\
Verdünnung & DSU $1: 10$, & DSU $1: 5$, \\
& PND1 $1: 10-1: 200$, & PND $1: 1-1: 20$, \\
& PND2 $1: 15$ & (keine weitere Verdünnung) \\
& $\rightarrow f_{\text {dil,tot. }}=1500-30000$ & $\rightarrow f_{\text {dil,tot. }}=5-100$ \\
VPR & Verdünnung und & nur Verdünnung \\
& Evaporation Tube $\left(350^{\circ} \mathrm{C}\right)$ & \\
Merkmal & PMP Konformität & Partikelgrößenklassierung \\
Verwendung in & bei allen Versuchen & bei ausgewählten \\
dieser Arbeit & & Versuchen \\
\hline
\end{tabular}

Tabelle A.2.: Gegenüberstellung der Eigenschaften der verwendeten Partikelzähler 


\section{Abbildungsverzeichnis}

2.1. Gemischbildungsprozess nach Samenfink [102 . . . . . . . . . . . 4 4

2.2. Elektromagnetisches Einspritzventil vom Typ Bosch EV14 sowie Spraywinkeldefinitionen 89 . . . . . . . . . . . . . . . . . . . 6 6

2.3. Einspritzstrategien mit beispielhafter Darstellung der elektrischen Ansteuerung für eine saugsynchrone Einspritzung . . . . . . . . . . . .

2.4. (a) Ohnesorge-Diagramm [80, 91] mit dem für diese Arbeit relevanten Bereich für SRE (blau) und zum Vergleich für BDE (grau)

(b) Zerfallsmechanismen nach Stahl [113] . . . . . . . . . . . . . . .

2.5. Einteilung der Sekundär-Zerfallsmechanismen nach Pilch u. Erdmann

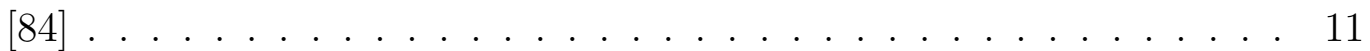

2.6. Phänomenologisches Modell einer Wandfilmströmung nach [110] . . . . 12

2.7. Normierte Tropfenlebensdauer als Funktion des Umgebungsdrucks und der Umgebungstemperatur nach Gartung [36 . . . . . . . . . . . . . 15

2.8. Verdunstungsvorgang eines n-Heptanwandfilms mit Tropfenbildung (oben) und eines Benzinwandfilms mit zerfließenden Tropfen (unten) [109] . . . 16

2.9. Experimentell bestimmte Verdunstungszeiten der Wandfilme aus isoOktan (links) und aus iso-Oktan, 3-Komponenten- Ersatzkraftstoffkraftstoff und ROZ 95 (rechts) $[109] \ldots$. . . . . . . . . . . . . . . . 17

2.10. Partikelentstehung nach Bockhorn [9] und Mayer [66] . . . . . . . . 19

2.11. Beispielhafte Rußbildung im ottomorischen Prozess mit Benzindirekteinspritzung nach Kufferath et al. [57] . . . . . . . . . . . . . .

3.1. Schema der Verdunstungskammer mit Systemgrenze in rot (links); Foto des Versuchsaufbaus (rechts) . . . . . . . . . . . . . .

3.2. Gemessener Kammerdruckverlauf für $T_{0}=93.5^{\circ} \mathrm{C}, p_{0}=1000 \mathrm{mbar}$ mit Injektor G sowie berechneten, thermodynamischen Grenzkurven . . . . 32

3.3. Schematische Darstellung des optischen Setups am Transparentmotor von oben (linke Bildhälte) und von vorne (rechte Bildhälfte) . . . . . .

3.4. Aufbau des Transparentmotors mit zwei PCO Dimax High-Speed Ka-

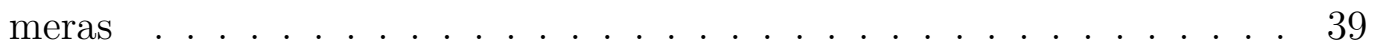

3.5. Beispiele zur Methodik der Wandfilmquantifizierung am Transparentmotor für alle drei Perspektiven (1-V, 1-L, 2-L) in vier Schritten . . . . 40

3.6. Beispiele für Analysen hinsichtlich der Auftretenswahrscheinlichkeit von Kraftstoffwandfilm anhand statistischer Auswertung von 10 Zyklen . . 41

3.7. Versuchsaufbau am Vollmotorprüfstand . . . . . . . . . . . . . . . . 43

3.8. Schema und Perspektiven der optischen Zugänge zur HSVE . . . . . . 44 
3.9. Beispiel zur statistischen Analyse von Partikelquellen im Brennraum: (a) rötliche Diffusionsflamme einer einzelnen Verbrennung links; (b) Häufigkeit für erkannte Diffusionsflammen von 40 Zyklen rechts . . . . . . . 46

4.1. Verdunstungsmessungen mit verschiedenen Kammerdrücken $\left(p_{0}\right)$ bei $T_{0}=93^{\circ} \mathrm{C}$ und konstanter Einspritzmasse $m_{i n j}=36 \mathrm{mg} \ldots \ldots$

4.2. Verdunstungsmessungen mit verschiedenen Injektor-Sprays bei konstanter Einspritzmasse $m_{i n j}=36 \mathrm{mg}$ bei 1000 mbar Kammerdruck . . . . . 51

4.3. Kennzahl zur Bewertung der Verdunstungsneigung verschiedener Sprays bei warmer Kammer (links) und kalter Kammer (rechts) . . . . . . . . 53

4.4. Ladungswechsel-Analyse bei $N=2000 \mathrm{~min}^{-1}, I M E P=2,5 \mathrm{bar}$ und $p_{S R}=600$ mbar am Vollmotor . . . . . . . . . . . . . . 54

4.5. Ladungswechsel-Analyse bei $N=2000 \mathrm{~min}^{-1}, I M E P=12 \mathrm{bar}$ und $p_{S R}=1100$ mbar am Vollmotor . . . . . . . . . . . . . . . 55

4.6. High-Speed Visualisierung der Rückströmung am Vollmotor mit cVI und Injektor A . . . . . . . . . . . . .

4.7. PN-Emissionen bei steigender Motorlast mit vorgelagerter Einspritzung (cVI), dargestellt als Lastschnitte bei konstanten Drehzahlen mit Injektor A und B (links) und als Kennfeld mit Injektor A (rechts) . . .

4.8. Gegenüberstellung von Verbrennungen mittels HSVE aus einer Messung mit niedriger Motorlast (links) und hoher Motorlast (rechts) mit Injektor B . . . . . . . . . . . . . . . . . . .

4.9. Fläche der Kraftstoffwandfilme an der Unterseite der Einlassventile (Perspektive ,1-V“) am Transparentmotor bei $\mathrm{N}=2000 \mathrm{~min}^{-1}$ und steigendem $p_{S R}$ mit Injektor B . . . . . . . . . . . . . . . . .

4.10. Variation der Steuerzeit Einlassventil öffnet (EVö) im Hinblick auf die Rückströmung in den Einlasskanal sowie auf PN mit cVI . . . . . . . . 61

4.11. Variationen des Einspritzende (EOI) bezüglich LW-OT für diverse Betriebspunkte mit Injektor B bei warmem Motor $\left(T_{\ddot{O} l}=93{ }^{\circ} \mathrm{C}\right) \quad \ldots . .662$

4.12. Gegenüberstellung von Diffusionsflammen bei vorgelagertem Timing (links) und saugsynchronem EinspritzTiming (rechts) mit Injektor B . . 64

4.13. Gegenüberstellung der Auftretenswahrscheinlichkeit von Wandfilm nach 10 Zyklen bei vorgelagerter $\left(\mathrm{EOI}=133^{\circ} \mathrm{KW}\right.$ v. LW-OT) und saugsynchroner Einspritzung (EOI $=-103^{\circ} \mathrm{KW}$ v. LW-OT) mit Injektor B und $p_{S R}=900$ mbar am Transparentmotor . . . . . . . . . . . . . .

4.14. Spezifischer Kraftstoffverbrauch und gasförmige Schadstoffemissionen in Abhängigkeit des Einspritztimings . . . . . . . . . . . . 66

4.15. Vorgehensweise zur reproduzierbaren Messung von Lastsprüngen bei zwangsgekühltem Motor . . . . . . . . . . . . . . . .

4.16. Vergleich der mittleren PN-Emissionen aus jeweils drei Lastsprüngen von $I M E P_{1}$ auf $I M E P_{2}$ bei warmem Motor mit $T_{\ddot{\mathrm{O}} l}=94{ }^{\circ} \mathrm{C}$ (links) und kaltem Motor mit $T_{\ddot{\mathrm{O} l}}=35^{\circ} \mathrm{C}$ (rechts) . . . . . . . . . . . . . .
Gegenüberstellung von Verbrennungen mittels HSVE mit warmem Motor (links) und kaltem Motor (rechts) . . . . . . . . . . . . .

4.17. Gegenüberstellung von Verbrennungen mittels HSVE mit warmem Mo- 
4.18. Gegenüberstellung der räumlichen Auftretenswahrscheinlichkeit von Diffusionsflammen mittels HSVE mit $N=2000 \mathrm{~min}^{-1}$ bei Erreichung der Ziellast von $I M E P_{2}=12$ bar mit deaktivierter LBK (links) und aktivierter LBK (rechts) mit Injektor B . . . . . . . . . . . . . . . .

4.19. Gegenüberstellung der Auftretenswahrscheinlichkeit von Wandfilm nach 10 Zyklen bei saugsynchroner Einspritzung (oVI) mit deaktivierter LBK (links) und aktiver LBK (rechts) mit Injektor B . . . . . . . . . . . . .

4.20. Berechnung der Weber-Zahl We sowie des kritischen Tropfendurchmessers $d_{\text {Tr.krit. }}$ mit Annahmen 11 auf Basis von Ladungswechselrechnungen bei $\mathrm{N}=2000 \mathrm{~min}^{-1}$, IMEP $=12$ bar mit Injektor B . . . . . . . . . .

4.21. Diffussionsflammen eines einzelnen Zyklus (links) und statistische Auswertung über 20 Zyklen (rechts) bei $I M E P_{2}=10$ bar mit Injektor G . 76

4.22. Gegenüberstellung der räumlichen Auftretenswahrscheinlichkeit von Diffusionsflammen im Lastbereich von $I M E P_{2}=10$ bar mit deaktivierter LBK (links) und aktivierter LBK (rechts) mit Injektor G . . . . . . 76

4.23. Parameterraum Targetingstudie . . . . . . . . . . . . . . 77

4.24. CAD-Visualisierungen der für diese Studie ausgewählten Spray-Auslegungen in der Seitenansicht (oben) und Draufsicht (unten) . . . . . . 78

4.25. PN-Emissionen gemittelt über drei Lastsprünge bei warmem Motor für drei Parameter-Kombinationen . . . . . . . . . . . . . . . . . 79

4.26. Gegenüberstellung von Spray-Targeting und Verbrennung von Injektor D und G mittels HSVE hinsichtlich Partikelentstehung bei warmem Motor 81

4.27. Gegenüberstellung der Auftretenswahrscheinlichkeit von Wandfilm nach 10 Zyklen bei vorgelagerter Einspritzung $(\mathrm{cVI})$ und LBK $=0$ mit Injektor D (links) und Injektor G (rechts) . . . . . . . . . . . .

4.28. Gegenüberstellung der räumlichen Auftretenswahrscheinlichkeit von Diffusionsflammen mit Injektor B und G bei $I M E P_{2}=12$ bar, oVI, LBK $=0$ und kaltem Motor . . . . . . . . . . . . . . .

4.29. Gegenüberstellung der Auftretenswahrscheinlichkeit von Wandfilm nach 10 Zyklen bei saugsynchroner Einspritzung (oVI) und LBK=0 mit Injektor B (links) und Injektor G (rechts) . . . . . . . . . . . . . . .

4.30. Kenndaten und CAD-Visualisierungen der Spray-Auslegungen der TwinSRE-Injektoren bei Rotation um $0^{\circ}$ („oben“) sowie der Single-Ver-

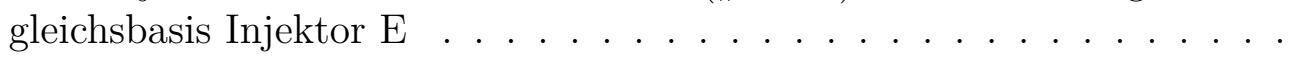

4.31. Variation des Spray-Targetings durch Rotation der Einbaulage am Beispiel von Injektor J . . . . . . . . . . . . . . . . . . . .

4.32. PN-Emissionen in Abhängigkeit des Spray-Targetings, variiert durch Rotation der Injektoren, bei IMEP $=12$ bar, $\mathrm{LBK}=0$ und warmem Motor . . . . . . . . . . . . . . . . . .

4.33. Gegenüberstellung der PN-Emissionen mit deaktivierter und aktivierter Ladungsbewegungsklappe in Abhängigkeit des durch Injektor-Rotation veränderten Spray-Targetings, bei IMEP $=12$ bar und kaltem Motor . 
4.34. Gegenüberstellung der Auftretenswahrscheinlichkeit von Diffusionsflammen bei IMEP $=12$ bar mit deaktivierter LBK (links) und aktivierter LBK (rechts) mit Injektor K und Sprayorientierung nach innnen . . . .

4.35. Skizzen zur Wechselwirkung der starken Ansaugströmung bei LBK $=1$ mit dem Single-Spray von Injektor E (links) und dem Twin-Spray mit Orientierung nach innen von Injektor K (rechts) . . . . . . . . . . . . 91

4.36. Einfluss des Kraftstoffdrucks $p_{K r}$ auf die Tröpfchendurchmesser des Sprays von Injektor E sowie auf die Partikelemissionen . . . . . . . . .

4.37. Partikelemissionen bei Variation des Massenaufteilungsfaktors einer Zweifach-Einspritzung bei warmem Motor (links) und kaltem Motor (rechts) mit Twin-Injektor J orientiert nach oben . . . . . . . . . . . . . . . 95

5.1. Übersicht der drei dominierenden Partikelquellen innerhalb des Brennraums mit Saugrohreinspritzung . . . . . . . . . . . . . . 97

5.2. Bewertung der PN-Quellen mit Saugrohreinspritzung [106] und Gegenüberstellung zur Direkteinspritzung nach Kufferath et al. [57]. 0 今 geringer Einfluss; $+++\hat{=}$ großer Einfluss . . . . . . . . . . . . . 98

A.1. Die ersten fünf Verbrennungen von Zylinder vier während eines Kaltstarts mit saugsynchroner Einspritzung und Injektor B bei fst $=2,4$. . 102

A.2. HC-Emission bei cVI im Bilanzzeitraum von $40 \mathrm{~s}$ während des Katalysatorheizens in Abhängigkeit von $\lambda$ und Injektorspray . . . . . . . . . 103

A.3. Deckblatt der Erfindungsmeldung . . . . . . . . . . . . . . . . . . . . . 104

A.4. Zusatznocken mit beispielhaftem Öffnungszeitpunkt . . . . . . . . . . . 106

A.5. Beispielhafte Öffnungszeitpunkte und Ventilerhebungskurven für Lösung 1 und $2 \ldots \ldots \ldots \ldots$. . . . . . . . . . . . . . . . . . . . .

A.6. Zusatz-Hub auf zweiter Nocke . . . . . . . . . . . . . . . . . . . . . 108

A.7. Gemischsättigungstemperaturen für Superkraftstoff [30, 107] . . . . . . 109

A.8. Einfluss der Gasdichte auf den Strahlzerfall (Primärzerfall) nach Reitz [90]. . . . . . . . . . . . . . . . . . . . . 110

A.9. Wandfilmtransportvorgänge nach Costanzo u. Heywood [16 für niedrige Gasgeschwindigkeit a) - c) und für hohe Gasgeschwindigkeit d) - f) . . . 110

A.10.Klassierung von PN- und PM-Emissionen aus Fahrzeugmessungen nach Sabathil [98] (Zwischenzeitlich ist der EU6 Grenzwert für die Partikelanzahlemissionen mit $6 \cdot 10^{11} \mathrm{\#} / \mathrm{km}$ bekannt und in Kraft) . . . . . . . 111

A.11.Auszug der HC-Emissionen eines Fahrzeugs mit SRE während eines FTP75-Fahrzyklus . . . . . . . . . . . . . . . . . 111

A.12.Zeitbasierte Daten von WLTC Fahrzyklusmessungen eines SRE-Fahrzeugs und eines BDE-Fahrzeugs bei verschiedenen Starttemperaturen

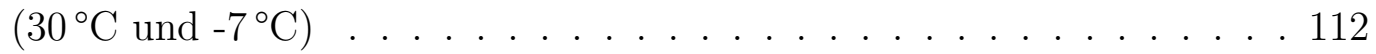

A.13.Vergrößerte Ansicht der Beispiele zur Methodik der Wandfilmquantifizierung am Transparentmotor für alle drei Perspektiven (1-V, 1-L, 2-L) in vier Schritten $(\mathrm{a}, \mathrm{b}, \mathrm{c}, \mathrm{d})$, siehe Kapitel $3.2 .2 \ldots \ldots$. . . . . . . . 113 
A.14.Kenndaten und CAD-Visualisierungen der Spray-Auslegungen der SingleSRE-Injektoren, siehe Kapitel 3.4 . . . . . . . . . . . . . . . . . . . . . 114

A.15.Visualisierung von Ansaugung und Verbrennung eines Arbeitsspiels mittels HSVE bei $N=2000 \mathrm{~min}^{-1}$, IMEP $=15,6$ bar und vorgelagerter Einspritzung mit EOI $=300^{\circ} \mathrm{KW}$. EVs (links) und saugsynchroner Einspritzung mit EOI $=48^{\circ} \mathrm{KW}$ v. LW-OT (rechts), siehe Kapitel 4.2.3 . 115

A.16.PN-Emissionen aller Single-Injektoren gemittelt über drei Lastsprünge bei warmem Motor für drei Parameter-Kombinationen . . . . . . . . . 116

A.17.PN-Emissionen aller Single-Injektoren gemittelt über drei Lastsprünge bei kaltem Motor für drei Parameter-Kombinationen . . . . . . . . . . 116

A.18.PN-Emissionen ausgewählter Single-Injektoren gemittelt über drei Lastsprünge bei kaltem Motor für drei Parameter-Kombinationen . . . . . . 117

A.19.Gegenüberstellung der Auftretenswahrscheinlichkeit von Diffusionsflammen von Injektor $\mathrm{G}$ und $\mathrm{C}$ mittels $\mathrm{HSVE}$ bei $I M E P_{2}=12 \mathrm{bar}$, cVI, $\mathrm{LBK}=0$ und warmem Motor, siehe Kapitel 4.2.6 . . . . . . . . 117

A.20.Gegenüberstellung der Auftretenswahrscheinlichkeit von Wandfilm nach 30 Zyklen bei vorgelagerter Einspritzung (cVI) und $L B K=0$ mit Injektor D (links) und Injektor G (rechts), siehe Kapitel 4.2.6 . . . . . . . 118

A.21.PN-Emissionen in Abhängigkeit des Spray-Targetings, variiert durch Rotation der Injektoren, bei kaltem Motor . . . . . . . . . . . . . . 118

A.22.Gasförmige Schadstoffemissionen und Restsauerstoff bei Variation des Kraftstoffdrucks $p_{K r}$ mit Injektor E, siehe Kapitel 4.2.8 . . . . . . . . . 119

A.23.Gegenüberstellung der räumlichen Auftretenswahrscheinlichkeit von Diffusionsflammen bei $I M E P=12$ bar mit deaktivierter LBK (links) und aktivierter LBK (rechts) mit Single-Injektor E, siehe Kapitel 4.2.7 . . . 120 


\section{Tabellenverzeichnis}

1.1. EU6 Schadstoffemissionsgrenzwerte für Ottomotoren [22] sowie NTE Limits (Not to exceed) für RDE $[2527 \ldots \ldots \ldots$

3.1. Überlagerte Prozesse während der Spray-Verdunstung . . . . . . . . . . 32

3.2. Technische Daten des Einzylinder-Transparentmotors . . . . . . . . . . 37

3.3. Am Transparentmotor untersuchte Bereiche (ROI) innerhalb des Brennraums .......................... 40

3.4. Technische Daten des Vollmotors . . . . . . . . . . . . . . . 42

3.5. Kenndaten der untersuchten Injektor-Spray Varianten bei $p_{K r}=3.0 \mathrm{bar} 48$

4.1. Kenndaten der ausgewählten Spray-Varianten bei $p_{K r}=3.0$ bar . . . . 78

A.1. Schadstoffemissionen im Bilanzzeitraum des Kaltstarts von sechs Sekunden bezüglich der EU6d-Grenzwerte (Bezugsstrecke WLTC) . . . . 103

A.2. Gegenüberstellung der Eigenschaften der verwendeten Partikelzähler . . 120 


\section{Literaturverzeichnis}

[1] Alkidas, Alex C.: Intake-Valve Temperature Histories During S.I. Engine Warm-Up. In: Vehicle Thermal Management Systems Conference \&6 Exposition. Nashville, TN, USA, 2001 (SAE Technical Paper 2001-01-1704). - DOI: http://dx.doi.org/10.4271/2001-01-1704

[2] Aquino, C.F.: Transient A/F Control Characteristics of the 5 Liter Central Fuel Injection Engine. In: SAE International Congress and Exposition. Detroit, MI, USA, Feb 1981 (SAE Technical Paper 810494). - DOI: https://doi.org/10.4271/810494. - ISSN 0148-7191

[3] Arcoumanis, C. ; Gavaises, M. ; French, B.: Effect of Fuel Injection Processes on the Structure of Diesel Sprays. In: International Congress 86 Exposition. Detroit, MI, USA, Feb 1997 (SAE Technical Paper 970799). - DOI: https://doi.org/10.4271/970799. - ISSN 0148-7191

[4] Arsie, Ivan ; Di Iorio, Silvana ; VACCARo, Salvatore: Experimental Characterization of Nanoparticles Emissions in a Port Fuel Injection Spark Ignition Engine. In: 10th International Conference on Engines \& Vehicles. Capri, Neapel, Italien, Sep. 2011 (SAE Technical Paper 2011-24-0208). - DOI: https://doi.org/10.4271/2011-24-0208. - ISSN 0148-7191

[5] AVL List GmbH: AVL M.O.V.E iS A new solution for the upcoming EU6c - Real Driving Emissions (RDE) legislation. Website. 2014. - URL https://www.avl.com/documents/10138/885965/AVL+M.O.V.E+iS+ Information. - Zugriffsdatum: 16.03.2020

[6] Badshah, Huzeifa ; Kittelson, David ; Northrop, William: Particle Emissions from Light-Duty Vehicles during Cold-Cold Start. In: SAE International Journal of Engines 9 (2016), Nr. 3, S. 1775-1785. - ISSN 1946-3944

[7] Baumgarten, Carsten: Mixture formation in internal combustion engines. Berlin : Springer-Verlag, 2006. - ISBN 3-540-30835-0

[8] Bellmann, Holger: Ermittlung der Kraftstoffwandfilmmasse im Saugrohr eines Ottomotors, Fortschritt-Berichte VDI / Reihe 12, Verkehrstechnik/Fahrzeugtechnik. Düsseldorf, Techn. Universität Braunschweig, Dissertation, 1995

[9] Bockhorn, Henning: Soot formation in combustion: Mechanisms and Models. Berlin : Springer-Verlag, 1994. - ISBN 978-3-642-85169-8 
[10] Brodkey, Robert S.: The phenomena of fluid motions. Reading, Massachusetts, USA : Addison-Wesley, 1967 (Series in Chemical Engineering)

[11] Buder, Andreas: Die Chemilumineszenz - Ihre Anwendung in der Konservierungsforschung bei der Untersuchung der Stabilität eines TriterpenNaturharzes, Hochschule der Bildenden Künste Dresden HFBK, Dissertation, 2017. - URL https://www.researchgate.net/publication/324030766_Die_ Chemilumineszenz___Ihre_Anwendung_in_der_Konservierungsforschung_ bei_der_Untersuchung_der_Stabilitat_eines_Triterpen-Naturharzes. Zugriffsdatum: 13.11 .2020

[12] Chan, Tak W. ; Meloche, Eric ; Kubsh, Joseph ; Rosenblatt, Deborah ; Brezny, Rasto ; Rideout, Greg: Evaluation of a Gasoline Particulate Filter to Reduce Particle Emissions from a Gasoline Direct Injection Vehicle. In: $S A E$ International Journal of Fuels and Lubricants Vol. 5 (2012), Nr. No. 3, S. $1277-$ 1290. - DOI: http://dx.doi.org/10.4271/2012-01-1727

[13] Chen, Longfei ; Liang, Zhirong ; Zhang, Xin ; Shuai, Shijin: Characterizing particulate matter emissions from GDI and PFI vehicles under transient and cold start conditions. In: Fuel 189 (2017), S. 131 - 140. - DOI: https://doi.org/10.1016/j.fuel.2016.10.055. - ISSN 0016-2361

[14] Chevalier, Alain ; Vigild, Christian W. ; Hendricks, Elbert: Predicting the Port Air Mass Flow of SI Engines in Air/Fuel Ratio Control Applications. In: SAE 2000 World Congress. Detroit, MI, USA, 2000 (SAE Technical Paper 2000-01-0260). - DOI: https://doi.org/10.4271/2000-01-0260. - ISSN 0148-7191

[15] Claret, Jose ; Lauer, Thomas ; Bobicic, Nikola ; Posselt, Andreas ; SCHLERFER, Joerg: Impact of the Injection and Gas Exchange on the Particle Emission of a Spark Ignited Engine with Port Fuel Injection. In: $W C X$ 17: SAE World Congress Experience. Detroit, MI, USA, 2017 (SAE Technical Paper 2017-01-0652). - DOI: http://doi.org/10.4271/2017-01-0652

[16] Costanzo, Vincent S. ; Heywood, John B.: Mixture Preparation Mechanisms in a Port Fuel Injected Engine. In: SAE Brasil Fuels \&s Lubricants Meeting. Rio de Janeiro, Brazil, 2005 (SAE Technical Paper 2005-01-2080). - DOI: http://dx.doi.org/10.4271/2005-01-2080

[17] Curtis, Eric W. ; Aquino, Charles F. ; Trumpy, David K. ; Davis, George C.: A New Port and Cylinder Wall Wetting Model to Predict Transient Air/Fuel Excursions in a Port Fuel Injected Engine. In: International Fuels \& Lubricants Meeting 65 Exposition. San Antonio, TX, USA, Mai 1996 (SAE Technical Paper 961186). - DOI: https://doi.org/10.4271/961186. - ISSN 0148-7191

[18] Dageförde, Helge ; Bertsch, Markus ; Kubach, Heiko ; Koch, Thomas: Reduktion der Partikelemissionen bei Ottomotoren mit Direkteinspritzung, MTZ - Motortechnische Zeitschrift. In: MTZ - Motortechnische Zeitschrift 76 (2015), Nr. 10, S. 86-93 
[19] Dageförde, Helge ; Kiefer, Arthur ; Samenfink, Wolfgang ; Wiese, Wolfram ; Kufferath, Andreas: Requirements for Spray and Tip Design of a Multihole Injector for DISI Engines. In: ICLASS 2015, 13th Triennial International Conference on Liquid Atomization and Spray Systems (2015), Aug., Nr. B13-237. - URL https://ilasseurope.org/events/13th-iclass-2015/. - Zugriffsdatum: 13.11 .2020

[20] Dageförde, Helge ; Koch, Thomas ; Beck, Kai W. ; SpIcher, Ulrich: Influence of Fuel Composition on Exhaust Emissions of a DISI Engine during Catalyst Heating Operation. In: SAE Int. J. Fuels Lubr. 6 (2013), S. 627-640

[21] Das europäische Parlament und DeR Europäische Rat: Verordnung (EG) Nr. 715/2007. In: Amtsblatt der Europäischen Union (2007), Nr. L 171. - URL http://data.europa.eu/eli/reg/2007/715/oj. - Zugriffsdatum: 14.11 .2020

[22] Das europäische Parlament und Der europäische Rat: Verordnung (EG) Nr. 692/2008. In: Amtsblatt der Europäischen Union (2008), Nr. L 199. - URL http://data. europa .eu/eli/reg/2008/692/oj. - Zugriffsdatum: 14.11 .2020

[23] Delphi Technologies: Worldwide emissions standards Passenger cars and light duty vehicles 2019 / 2020. 2019. - URL https://www.delphi.com/sites/default/files/2019-05/2019-2020\% 20Passenger $\% 20$ Car $\% 20 \& \% 20$ Light-Duty\%20Vehicles.pdf. - Zugriffsdatum: 12.11 .2020

[24] Die europäische Kommision: Verordnung (EU) 2016/427. In: Amtsblatt der Europäischen Union (2016), Nr. L 82. - URL http://data.europa.eu/eli/ reg/2016/427/oj, - Zugriffsdatum: 14.11.2020

[25] Die europäische Kommision: Verordnung (EU) 2018/1832. In: Amtsblatt der Europäischen Union (2018), Nr. L 301. - URL http://data.europa.eu/ eli/reg/2018/1832/oj. - Zugriffsdatum: 13.11.2020

[26] Die europäische Kommission: Verordnung (EU) 2017/1151. In: Amtsblatt der Europäischen Union (2017), Nr. L 175. - URL http://data.europa.eu/ eli/reg/2017/1151/oj. - Zugriffsdatum: 14.11.2020

[27] Die europäische Kommission: Verordnung (EU) 2017/1154. In: Amtsblatt der Europäischen Union (2017), Nr. L 175. - URL http://data.europa.eu/ eli/reg/2017/1154/oj. - Zugriffsdatum: 14.11.2020

[28] Eastwood, Peter: Particulate emissions from vehicles. Chichester, England : John Wiley \& Sons Ltd., 2008 (Wiley-Professional engineering publishing series).

- ISBN 978-0-470-72455-2 
[29] EBNER, Joachim: Einfluss von Druckgradienten in der Gasströmung auf die Dynamik schubspannungsgetriebener Wandfilme, Universität Karlsruhe (TH), Dissertation, 2004

[30] Eichlseder, Helmut ; Klüting, Manfred ; Piock, Walter F.: Grundlagen und Technologien des Ottomotors, Der Fahrzeugantrieb. Wien : Springer Verlag, 2008. - ISBN 978-3-211-47104-3

[31] ElsÄssER, Alfred: Kraftstoffaufbereitung in Verbrennungskraftmaschinen: Grundlagen der Strömung schubspannungsgetriebener Wandfilme, Univ. Karlsruhe, Dissertation, 1998

[32] EPA: Greenhouse Gas Emissions, Fuel Economy, and Technology since 1975. In: EPA Automotive Trends Report (2019), Nr. EPA-420-R-19-002

[33] Ericsson, Per ; Samson, Andreas: Characterization of Particulate Emissions Propagating in the Exhaust Line for Spark Ignited Engines. In: SAE 2009 Powertrains Fuels and Lubricants Meeting. Florenz, Italien, 2009 (SAE Technical Paper 2009-01-2654). - DOI: http://dx.doi.org/10.4271/2009-01-2654

[34] EuropäIsches Parlament: Genehmigung und Marktüberwachung von Kraftfahrzeugen und Kraftfahrzeuganhängern sowie von Systemen, Bauteilen und selbständigen technischen Einheiten für diese Fahrzeug. In: Ordentliches Gesetzgebungsverfahren: Erste Lesung (2017), Nr. (P8-TA-2017-0097). - URL http:// WwW .europarl.europa.eu/doceo/document/TA-8-2017-0097_EN.pdf. - Zugriffsdatum: 13.11 .2020

[35] Gansert, Klaus-Peter: Laserinduzierte Tracerfluoreszenz-Untersuchungen zur Gemischaufbereitung am Beispiel des Ottomotors mit Saugrohreinspritzung, Universität Bayreuth, Dissertation, 2003

[36] Gartung, K.: Modellierung der Verdunstung realer Kraftstoffe zur Simulation der Gemischbildung bei Benzindirekteinspritzung, Universität Bayreuth, Dissertation, 2008

[37] Geringer, B. ; Hofmann, P. ; Hackl, B. ; Urbanek, M. ; Samenfink, W. ; Frank, M. ; Gerhardt, J. ; Gesk, M.: Neue Wege bei der Injektorauswahl und der Startabstimmung von Ottomotoren mit Saugrohreinspritzung. In: $2 \%$. Internationales Wiener Motorensymposium 2006 Bd. 622. Düsseldorf : VDI Verlag GmbH, 2006, S. 340-363. - ISBN 3-18-362212-2

[38] Gnanam, G. ; Habibi, S. R. ; Burton, R. T. ; Sulatisky, M. T.: Neural network control of air-to-fuel ratio in a bi-fuel engine. In: IEEE Transactions on Systems, Man, and Cybernetics, Part C (Applications and Reviews) 36 (2006), Nr. 5, S. 656-667. - ISSN 1094-6977 
[39] Godsave, G.A.E.: Studies of the combustion of drops in a fuel spray - the burning of single drops of fuel. In: Fourth Symposium (International) on Combustion. MIT Cambridge, Massachusetts, USA, 1953, S. 818 - 830. - DOI: https://doi.org/10.1016/S0082-0784(53)80107-4. - ISSN 0082-0784

[40] Gold, M. R. ; Arcoumanis, C ; Whitelaw, J. H. ; Gaade, J ; Wallace, S: Mixture preparation strategies in an optical four-valve port-injected gasoline engine. In: International Journal of Engine Research 1 (2000), Nr. 1, S. 41-56. - DOI: https://doi.org/10.1243

[41] Graf, Gerald ; Kiefer, A. ; Leder, M.: Operation strategy and control of a dual-injectionsystem for gasoline engines. In: BARGende, Michael (Hrsg.) ; Reuss, Hans-Christian (Hrsg.) ; Wiedemann, Jochen (Hrsg.): 17. Internationales Stuttgarter Symposium. Wiesbaden : Springer Fachmedien, 2017, S. 211-225. - URL https://doi.org/10.1007/978-3-658-16988-6_22. - Zugriffsdatum: 13.11.2020. - ISBN 978-3-658-16988-6

[42] Gupta, Tarun ; Kothari, Abhishek ; Srivastava, Dhananjay K. ; Agarwal, Avinash K.: Measurement of number and size distribution of particles emitted from a mid-sized transportation multipoint port fuel injection gasoline engine. In: Fuel 89 (2010), Nr. 9, S. 2230-2233. - ISSN 0016-2361

[43] Hemminger, Sebastian M.: Optimierung der Gemischbildung und Verbrennung von Hochleistungsottomotoren zur Einhaltung zukünftiger Emissionsgrenzwerte., Universität Stuttgart, Dissertation, 2014

[44] Hermann, Andreas: Modellbildung für die 3D-Simulation der Gemischbildung und Verbrennung in Ottomotoren mit Benzin-Direkteinspritzung, Universität Karlsruhe (TH), Dissertation, 2008

[45] Heyse, J. ; Schatz, F. ; Ader, B. ; Schlerfer, J.: Electroformed multilayer orifice plate for improved fuel injection characteristics: Eine mehrlagige Einspritzduesenoeffnung mit verbesserten Einspritzkenngroessen. In: SAE International Congress and Exposition. Detroit, MI, USA, 1997 (SAE Technical Paper 971070). - DOI: https://doi.org/10.4271/971070

[46] Heywood, John B.: Internal combustion engine fundamentals. New York : McGraw-Hill Education, 1988. - ISBN 007028637X

[47] Himmelsbach, Johann: Zweiphasenströmungen mit schubspannungsgetriebenen welligen Flüssigkeitsfilmen in turbulenter Heißluftströmung : meßtechnische Erfassung und numerische Beschreibung, Universität Karlsruhe, Dissertation, 1992

[48] Hoffmann, Armin: Aufbau und Inbetriebnahme eines Prüfstandes zur experimentellen Bewertung von PFI-Injektoren im Einlasskanal sowie Bewertung der Injektoren anhand einer Messkampagne, Universität Stuttgart, Institut für Verbrennungsmotoren und Kraftfahrwesen, Masterthesis, 2016 
[49] Horiba Automotive Test Systems: OBS-One PN Brochure. Website. 2016. - URL https://static.horiba.com/fileadmin/Horiba/ Products/Automotive/Emission_Measurement_Systems/OBS-ONE/OBS-ONE_ PN/OBS-ONE_PN_Brochure_English.pdf. - Zugriffsdatum: 16.03.2020

[50] Horiba Automotive Testing Systems: On-board Emissions Measurement System OBS-One. Website. 2017. - URL https://static.horiba.com/fileadmin/Horiba/Products/Automotive/ Emission_Measurement_Systems/OBS-ONE/OBS-ONE_Brochure_English.pdf. - Zugriffsdatum: 16.03.2020

[51] Hung, David L. ; Harrington, David L. ; Gandhi, Anand H. ; Markle, Lee E. ; Parrish, Scott E. ; Shakal, Joseph S. ; Sayar, Hamid ; Cummings, Steven D. ; Kramer, Jason L.: Gasoline Fuel Injector Spray Measurement and Characterization - A New SAE J2715 Recommended Practice. In: SAE 1 (2008), Nr. 1, S. 534-548

[52] Ikoma, Takuya ; Abe, Shizuo ; Sonoda, Yukihiro ; Suzuki, Hisao ; SuzuKI, Yuichi ; BASAKI, Masatoshi: Development of V-6 3.5-liter Engine Adopting New Direct Injection System. In: SAE 2006 World Congress 63 Exhibition. Detroit, MI, USA, 2006 (SAE Technical Paper 2006-01-1259). - DOI: http://dx.doi.org/10.4271/2006-01-1259

[53] KhaleK, Imad A. ; Bougher, Thomas ; Jetter, Jeff J.: Particle Emissions from a 2009 Gasoline Direct Injection Engine Using Different Commercially Available Fuels. In: SAE 2010 Powertrains Fuels 8 Lubricants Meeting. San Diego, CA, USA, 2010 (SAE Technical Paper 2010-01-2117). - DOI: https://doi.org/10.4271/2010-01-2117

[54] Kim, Hoisan ; Yoon, Suckju ; XIE, Xing-Bin ; LaI, Ming-Chia ; Quelhas, Sergio ; Boyd, Richard ; Kumar, Naresh ; Moran, Carilee: Effects of Injection Timings and Intake Port Flow Control on the In-Cylinder Wetted Fuel Footprints during PFI Engine Startup Process. In: 2005 SAE Brasil Fuels 86 Lubricants Meeting. Rio de Janeiro, Brazil, 2005 (SAE Technical Paper 200501-2082). - DOI: https://doi.org/10.4271/2005-01-2082. - ISSN 0148-7191

[55] Knauer, Markus ; Niessner, Reinhard ; Schuster, Michael: StrukturReaktivitäts-Korrelation von Dieselruß und Charakterisierung von PAHs und Carbonylen im Abgas von Biokraftstoffen, Technische Universität München, Dissertation, 2010

[56] Knorsch, Tobias ; Rogler, Philipp ; Miller, Maximilian ; Wiese, Wolfram: On the Evaluation Methods for Systematic Further Development of DirectInjection Nozzles. In: SAE 2016 International Powertrains, Fuels \& Lubricants Meeting. Baltimore, USA, 2016 (SAE Technical Paper 2016-01-2200). - DOI: https://doi.org/10.4271/2016-01-2200. - ISSN 0148-7191 
[57] Kufferath, A. ; Wiese, W. ; Samenfink, W. ; Dageförde, H. ; Knorsch, T. ; Jochmann, P.: Assessment of feasible system solutions for future particle emission requirements. In: Fuel Systems for IC Engines Conference. London, England : IMechE, 2015, S. 79-106

[58] Kulzer, Andre ; LeJsek, David: BeCAT - Bosch Engine Combustion Analysis Tool - User Manual. Stuttgart : Robert Bosch GmbH, 2010

[59] LANG, K.: Reducing cold start hydrocarbon emissions from port fuel injected spark ignition engines with improved management of hardware $\&$ controls, Massachusetts Institute of Technology, Dissertation, 2006

[60] LANG, Manuel: Thermodynamische Analyse von Einspritzvorgängen zur Bewertung der Verdunstungseigenschaften von Kraftstoffsprays, Hochschule Esslingen, Fakultät Fahrzeugtechnik, Bachelorthesis, 2017

[61] Lefebvre, A.H. ; McDoneld, V.G.: Atomization and Sprays. Second Edition. Boca Raton, FL, USA : CRC Press, 2017. - ISBN 978-1-4987-3626-8

[62] Lenz, Hans P.: Die Verbrennungskraftmaschine. Bd. Neue Folge, 6: Gemischbildung bei Ottomotoren. Wien : Springer Verlag, 1990. - ISBN 3211821937

[63] MaIer, Thomas ; Kiwull, Bettina: PN-Messverfahren: Untersuchung und Bewertung verschiedener Methoden der Partikelanzahl Messung; Abschlussbericht Vorhaben Nr. 1073 / Forschungsvereinigung Verbrennungskraftmaschinen. Frankfurt a.M., 2014. - Forschungsbericht. Heft 1049

[64] Maloney, Peter J.: An Event-Based Transient Fuel Compensator with Physically Based Parameters. In: International Congress 8 Exposition. Detroit, MI, USA, 1999 (SAE Technical Paper 1999-01-0553). - DOI: https://doi.org/10.4271/1999-01-0553. - ISSN 0148-7191

[65] Mansurov, Z. A.: Soot Formation in Combustion Processes (Review). In: Combustion, Explosion and Shock Waves 41 (2005), Nr. 6. - DOI: https://doi.org/10.1007/s10573-005-0083-2. - ISSN 1573-8345

[66] MayeR, Klaus: Pyrometrische Untersuchung der Verbrennung in Motoren mit Common-Rail-Direkteinspritzung mittels einer erweiterten Zwei-FarbenMethode, Forschungsberichte aus dem Institut für Kolbenmaschinen der Universität Karlsruhe (TH), Universität Karlsruhe (TH), Dissertation, 2000

[67] McGee, J. ; Curtis, Eric W. ; Russ, S. ; Lavoie, G.: The Effects of Port Fuel Injection Timing and Targeting on Fuel Preparation Relative to a PreVaporized System. In: International Fuels \& Lubricants Meeting $\&$ Exposition. Paris, Frankreich, 2000 (SAE Technical Paper 2000-01-2834). - DOI: http://dx.doi.org/10.4271/2000-01-2834 
[68] Merker, Guenter P.: Grundlagen Verbrennungsmotoren: Simulation der Gemischbildung, Verbrennung Schadstoffbildung und Aufladung. 4. Auflage. Wiesbaden : Vieweg + Teubner, 2009. - ISBN 978-3-8348-9344-4

[69] Merola, S. S. ; Sementa, P. ; Tornatore, C. ; Vaglieco, B. M.: Effect of Injection Phasing on Valves and Chamber Fuel Deposition Burning in a PFI Boosted Spark-Ignition Engine. In: SAE Int. J. Fuels Lubr. 1 (2008), S. 192-200. - DOI: http://dx.doi.org/10.4271/2008-01-0428

[70] Merola, S. S. ; Sementa, P. ; Tornatore, C. ; Vaglieco, B. M.: Effect of fuel injection strategies on the combustion process in a PFI boosted SI engine. In: International Journal of Automotive Technology 10 (2009), Nr. 5, S. 545-553. - DOI: http://dx.doi.org/10.1007/s12239-009-0063-8. - ISSN 1976-3832

[71] Merola, S. S. ; Vaglieco, B. M. ; Formisano, G. ; Lucignano, G. ; MaStrangelo, G.: Flame Diagnostics in the Combustion Chamber of Boosted PFI SI Engine. In: 8th International Conference on Engines for Automobiles. Capri, Neapel, Italien, 2007 (SAE Technical Paper 2007-24-0003). - DOI: http://dx.doi.org/10.4271/2007-24-0003

[72] Merola, Simona ; Sementa, Paolo ; Tornatore, Cinzia ; Carmignani, Luca ; Di Palma, Stefano: Fuel Injection Effect on In-cylinder Formation and Exhaust Emission of Particulate from a 4-Stroke Engine for 2-Wheel Vehicles. In: SAE 2010 World Congress \& Exhibition. Detroit, MI, USA, 2010 (SAE Technical Paper 2010-01-0354). - DOI: http://dx.doi.org/10.4271/2010-01-0354

[73] Merola, Simona S. ; Vaglieco, Bianca M.: Optical investigations of fuel deposition burning in ported fuel injection (PFI) spark-ignition (SI) engine. In: Energy 34 (2009), Nr. 12, S. 2108-2115. - DOI: https://doi.org/10.1016/j.energy.2008.08.018. - ISSN 0360-5442

[74] Meyer, R. ; Heywood, J. B.: Liquid Fuel Transport Mechanisms into the Cylinder of a Firing Port-Injected SI Engine During Start Up. In: International Congress 83 Exposition. Detroit, MI, USA, 1997 (SAE Technical Paper 970865), S. 87-102. - DOI: https://doi.org/10.4271/970865

[75] Meyer, Robert ; Heywood, John B.: Effect of Engine and Fuel Variables on Liquid Fuel Transport into the Cylinder in Port-Injected SI Engines. In: International Congress \& Exposition. Detroit, MI, USA, 1999 (SAE Technical Paper 1999-01-0563). - DOI: https://doi.org/10.4271/1999-01-0563. - ISSN 0148-7191

[76] Meyer, Robert ; Yilmaz, Ertan ; Heywood, John B.: Liquid Fuel Flow in the Vicinity of the Intake Valve of a Port-Injected SI Engine. In: International Fall Fuels and Lubricants Meeting and Exposition. San Francisco, CA, USA, 1998 (SAE Technical Paper 982471). - DOI: https://doi.org/10.4271/982471. ISSN 0148-7191 
[77] Ministry of Environmental Protection - General Administration of Quality Supervision, Inspection And Quarantine: GB 18352.6-2016: Limits and measurement methods for emissions from light-duty vehicles (China 6). In: National Standard of the People's Republic of China (2016), Nr. ICS 13.040.50; Z 64

[78] Mock, Peter ; Bieker, Georg ; Tietge, Uwe ; Rodriguez, Felipe: European vehicle market statistics 2019/2020. In: ICCT Report (2019). - URL https://theicct.org/sites/default/files/publications/ European_vehicle_market_statistics_20192020_20191216.pdf. - Zugriffsdatum: 13.11.2020

[79] National Institute of Standards and Technology: NIST Chemistry WebBook. Website. 2019. - URL https://webbook.nist.gov/chemistry/. Zugriffsdatum: 16.03.2020

[80] Ohnesorge, W. v.: Die Bildung von Tropfen an Düsen und die Auflösung flüssiger Strahlen. In: Zeitschrift für angewandte Mathematik und Mechanik 16 (1936), Nr. 6, S. 355-358

[81] Onder, Christopher H. ; Roduner, Christian A. ; Simons, Michael R. ; Geering, Hans P.: Wall-Wetting Parameters Over the Operating Region of a Sequential Fuel-Injected SI Engine. In: International Congress \& Exposition. San Francisco, CA, USA, 1998 (SAE Technical Paper 980792). - DOI: https://doi.org/10.4271/980792. - ISSN 0148-7191

[82] Pauer, Thomas ; Yilmaz, Hakan ; Zumbrägel, Joachim ; Schünemann, Erik: New Generation Bosch Gasoline Direct-injection Systems. In: MTZ worldwide 78 (2017), Nr. 7, S. 16-23. - ISSN 2192-9114

[83] Petit, Bertrand ; Boiarciuc, Andrei ; Radenac, Erwan ; Delahaye, Laurent ; FLOCH, Alain: PN Formation Mechanism and Countermeasures with the Spray Design on Port Fuel InjectionSI Engine. In: WCX World Congress Experience. Detroit, MI, USA, 2018 (SAE Technical Paper 2018-01-1417). - DOI: https://doi.org/10.4271/2018-01-1417. - ISSN 0148-7191

[84] Pilch, M. ; Erdman, C.A.: Use of breakup time data and velocity history data to predict the maximum size of stable fragments for acceleration-induced breakup of a liquid drop. In: International Journal of Multiphase Flow 13 (1987), Nr. 6, S. 741 - 757. - ISSN 0301-9322

[85] Pischinger, Rudolf ; Klell, Manfred ; Sams, Theodor: Thermodynamik der Verbrennungskraftmaschine. Wien : Springer Verlag, 2009. - ISBN 978-3-21199276-0

[86] Posselt, Andreas ; Mai, Arthur ; Leder, Martin ; Salvio, Rodrigo ; Coletto, Tulio: Advanced PFI: The Economical, High-Performance Systems Approach. In: 24th SAE Brasil International Congress and Display. 
Sao Paulo, Brasilien, 2015 (SAE Technical Paper 2015-36-0392). - DOI: https://doi.org/10.4271/2015-36-0392. - ISSN 0148-7191

[87] RAAB, Frank: Untersuchungen zur Reduzierung von KohlenwasserstoffEmissionen im Kaltstart von Ottomotoren mit Saugrohreinspritzung. Universitätsbibliothek, Otto-von-Guericke-Universität Magdeburg, Dissertation, 2007

[88] Rat der Europäischen Gemeinschaften: RICHTLINIE DES RATES vom 20. März 1970 zur Angleichung der Rechtsvorschriften der Mitgliedstaaten über Maßnahmen gegen die Verunreinigung der Luft durch Emissionen von Kraftfahrzeugen (70/220/EWG). (1970)

[89] ReIf, Konrad ; VIEWEG, Springer (Hrsg.): Ottomotor-Management. 4. Wiesbaden : Springer Fachmedien, 2014 (Bosch Fachinformation Automobil). - ISBN 978-3-8348-1416-6

[90] ReItz, Rolf: Atomization and other breakup regimes of a liquid jet, Princeton University, Dissertation, 1978

[91] Reitz, Rolf ; V. Bracco, F: Mechanisms of breakup of round liquid jets. In: Encyclopedia of Fluid Mechanics 3 (1986), S. 233-249

[92] Robert Bosch GmbH: Benzin-Saugrohreinspritzung - Die wirtschaftliche Lösung für bedarfsgerechte Leistung. Website. 2017. - URL https://www.bosch-mobility-solutions. de/de/produkte-und-services/pkw-und-leichte-nutzfahrzeuge/ antriebssysteme/benzin-saugrohreinspritzung/. - - Zugriffsdatum: 16.03.2020

[93] Rothe, Dieter: Physikalische und chemische Charakterisierung der Rußpartikelemission von Nutzfahrzeugdieselmotoren und Methoden zur Emissionsminderung, TU München, Dissertation, 2006

[94] Rottenkolber, G.: Ottomotoren im Kaltstart: laseroptische Messverfahren zur Charakterisierung des Kraftstofftransports, Universität Karlsruhe, Dissertation, 2001

[95] Rottenkolber, Gregor ; Dullenkopf, Klaus ; Koelmel, Armin ; WitTIG, Sigmar: Äußere Gemischbildung bei instationärem Motorbetrieb. Abschlußbericht zum FVV-Vorhaben Nr. 650: Instationäre Gemischbildung. Frankfurt, 1999. - Forschungsbericht. - 30 S. (FVV-Heft. 687.)

[96] Ruschel, Yvonne: Größenverteilung und Zusammensetzung von Dieselrußpartikeln beim Einsatz von Biodiesel im Vergleich zu unterschiedlichen Dieselkraftstoffen, TU Braunschweig, Dissertation, 2010

[97] Russ, S. ; Stevens, J. ; Aquino, C. ; Curtis, E. ; Fry, J.: The Effects of Injector Targeting and Fuel Volatility on Fuel Dynamics in a PFI Engine During Engine Warm-up: Part I - Experimental Results. In: International Fall 
Fuels and Lubricants Meeting and Exposition. San Francisco, CA, USA, 1998 (SAE Technical Paper 982518). - DOI: https://doi.org/10.4271/982518. - ISSN 0148-7191

[98] Sabathil, Daniel ; Koenigstein, Achim ; Schaffner, Peter ; Fritzsche, Jan ; DoeHLer, Arndt: The Influence of DISI Engine Operating Parameters on Particle Number Emissions. In: SAE 2011 World Congress \&6 Exhibition. Detroit, MI, USA, 2011 (SAE Technical Paper 2011-01-0143). - DOI: http://dx.doi.org/10.4271/2011-01-0143

[99] SAE: Gasoline Fuel Injector Spray Measurement and Characterization. 400 Commonwealth Drive, Warrendale, PA 15096-0001 : SAE International, 2007 (Ground Vehicle Standard J2715-200703). - URL https://doi.org/10.4271/ J2715_200703. - Zugriffsdatum: 13.11.2020

[100] Samenfink, W. ; Schlerfer, J. ; Joos, K. ; Weining, H. K. ; Holder, E. ; B., Heel: Unterschreitung der ULEV-Grenzwerte an einem DC-Versuchsmotor - Beitrag einer Start- und Nachstartoptimierung. In: 8. Aachener Kolloquium Fahrzeug- und Motorentechnik. Aachen, 1999, S. 253-273

[101] Samenfink, Wolfgang: Grundlegende Untersuchung zur Tropfeninteraktion mit schubspannungsgetriebenen Wandfilmen, Universität Karlsruhe, Dissertation, 1997

[102] Samenfink, Wolfgang: Grundschulung Gemischaufbereitung und Brennverfahren. 2003. - Bosch-interne Schulungsunterlagen

[103] Samenfink, Wolfgang ; Albrodt, Hartmut ; Frank, Michael ; Gesk, Markus ; Melsheimer, Anja ; Thurso, Jens ; MAtt, Martin: Strategies to Reduce HC-Emissions During the Cold Starting of a Port Fuel Injected Gasoline Engine. In: SAE 2003 World Congress \&f Exhibition. Detroit, MI, USA, 2003 (SAE Technical Paper 2003-01-0627). - DOI: https://doi.org/10.4271/2003-01-0627. - ISSN 0148-7191

[104] Samenfink, Wolfgang ; Hallmann, Mathias ; Elsässer, Alfred ; Wittig, Sigmar: Secondary breakup of liquid droplets: experimental investigation for a numerical description. In: Proceedings on the 6th International Conference on Liquid Atomization and Spray Systems. Rouen, 1994, S. 156

[105] Schück, Claudius ; Koch, Thomas ; Samenfink, Wolfgang ; Schuenemann, Erik ; TAFEL, Stephan ; TOWAE, Oliver: Optical Investigations of Soot Formation Mechanisms and Possible Countermeasures on a Turbocharged Port Fuel Injection SI Engine. In: SAE Int. J. Engines 9 (2016), S. 2010-2021. - DOI: https://doi.org/10.4271/2016-01-2163. - ISSN 1946-3936

[106] Schück, Claudius ; Samenfink, Wolfgang ; Schünemann, Erik ; Tafel, Stephan ; TowAE, Oliver ; KocH, Thomas: Analysis of particulate number 
emissions during dynamic load changes for port fuel injection on an optically accessible, turbocharged four-cylinder spark ignition engine. In: International Journal of Engine Research 19 (2018), Nr. 1, S. 78-85. - DOI: https://doi.org/10.1177/1468087417731044

[107] Scherenberg, D.: Einfluss der Gemischaufbereitung auf die Emissionen beim Start und Warmlauf sowie beim Uebergang von Last in Schubbetrieb eines Ottomotors bei verschiedenen Motortemperaturen, Universität Karlsruhe (TH), Dissertation, 1979

[108] Schuenemann, Erik ; Muench, Kai-Uwe ; Leipertz, Alfred: Interaction of Airflow and Injected Fuel Spray Inside the Intake Port of a Six Cylinder Four Valve SI Engine. In: International Fuels \& Lubricants Meeting \& Exposition. Tulsa, Oklahoma, USA, 1997 (SAE Technical Paper 972984). - DOI: https://doi.org/10.4271/972984. - ISSN 0148-7191

[109] Schulz, Florian: Untersuchung der Wandfilmbildung bei der Benzindirekteinspritzung unter Nutzung optischer Messverfahren, Micro-macro transactions, Otto-von-Guericke-Universität Magdeburg, Dissertation, 2016

[110] Senda, Jiro ; Ohnishi, Masanori ; Takahashi, Tomohiro ; Fujimoto, Hajime ; Utsunomiya, Atsushi ; Wakatabe, Michio: Measurement and Modeling on Wall Wetted Fuel Film Profile and Mixture Preparation in Intake Port of SI Engine. In: International Congress \& Exposition. Detroit, MI, USA, 1999 (SAE Technical Paper 1999-01-0798). - DOI: https://doi.org/10.4271/1999-01-0798. - ISSN 0148-7191

[111] Shin, Younggy ; Min, Kyoungdoug ; Cheng, Wai K.: Visualization of Mixture Preparation in a Port-Fuel Injection Engine During Engine Warm-up. In: 1995 SAE International Fall Fuels and Lubricants Meeting and Exhibition. Toronto, Canada, 1995 (SAE Technical Paper 952481). - DOI: https://doi.org/10.4271/952481. - ISSN 0148-7191

[112] Simon, N.: Kraftstoffwandfilmuntersuchungen im Saugrohr des Ottomotors mit Zentraleinspritzung. In: Fortschrittberichte VDI Bd. Reihe 12, Nr. 141. Düsseldorf : VDI-Verlag, 1990, S. 335-362

[113] Stahl, Martin: Experimentelle und numerische Untersuchung des primaeren Strahlzerfalls von Druckzerstaeubern. Darmstadt, Technische Universität, Dissertation, 2008

[114] Stengele, Jörg F.: Tropfenverdunstung in Hochdruckatmosphäre, Universität Karlsruhe, Dissertation, 1998

[115] Stojkovic, Boris D. ; Fansler, Todd D. ; Drake, Michael C. ; Sick, Volker: High-speed imaging of $\mathrm{OH}^{*}$ and soot temperature and concentration in a stratified-charge direct-injection gasoline engine. In: Proceedings of the Combustion Institute 30 (2005), Nr. 2, S. 2657 - 2665. - ISSN 1540-7489 
[116] Su, Jianye ; Lin, Weiyang ; Sterniak, Jeff ; Xu, Min ; Bohac, Stanislav V.: Particulate Matter Emission Comparison of Spark Ignition Direct Injection (SIDI) and Port Fuel Injection (PFI) Operation of a Boosted Gasoline Engine. In: Journal of Engineering for Gas Turbines and Power 136 (2014), Nr. 9, S. 091513-091513. - DOI: http://dx.doi.org/10.1115/1.4027274. - ISSN 07424795

[117] VDI E.V. (Hrsg.): VDI-Wärmeatlas. 11. Auflage. Berlin : Springer Vieweg, 2013. - ISBN 978-3-642-19980-6

[118] Wang, Le: Vergleich und Analyse der beiden Brennverfahren an einem Ottomotor mit kombinierten Benzin-Einspritzsystemen aus Saugrohr- und Direkteinspritzung hinsichtlich Wirkungsgrad und Emissionen. Karlsruhe, Karlsruher Institut für Technologie (KIT), Institut für Kolbenmaschinen (IFKM), Masterthesis, 2015

[119] WeBer, Daniel: Flash Boiling bei Ottomotoren mit Benzindirekteinspritzung: Experimentelle Untersuchungen, Modellierung und numerische Simulation, Technische Universität Darmstadt, Dissertation, 2016

[120] Wensing, Michael ; Münch, Kai-Uwe ; LeIPertz, Alfred: Characteristics and Application of Gasoline Injectors to SI Engines by Means of Measured Liquid Fuel Distributions. In: International Fuels $\&$ Lubricants Meeting $\&$ Exposition. Tulsa, Oklahoma, USA, 1997 (SAE Technical Paper 972947). - DOI: https://doi.org/10.4271/972947. - ISSN 0148-7191

[121] Whelen, D. E. ; Kelly-Zion, P. L. ; Lee, C.F. ; Peters, J. E. ; White, R. A.: Back-Flow Atomization in the Intake Port of Spark Ignition Engines. In: International Fuels $\&$ Lubricants Meeting $\&$ Exposition. Tulsa, Oklahoma, USA, 1997 (SAE Technical Paper 972988). - DOI: https://doi.org/10.4271/972988. ISSN 0148-7191

[122] WierzBA, A.: Deformation and breakup of liquid drops in a gas stream at nearly critical Weber numbers. In: Experiments in Fluids 9 (1990), Nr. 1, S. 59-64.ISSN 1432-1114

[123] Witze, Peter O. ; Green, Robert M.: Comparison of Single and Dual Spray Fuel Injectors During Cold Start of a PFI Spark Ignition Engine Using Visualization of Liquid Fuel Films and Pool Fires. In: Powertrain \& Fluid Systems Conference 85 Exhibition. San Antonio, TX, USA, 2005 (SAE Technical Paper 2005-01-3863). - DOI: https://doi.org/10.4271/2005-01-3863. - ISSN 0148-7191

[124] XiaO, Guowei ; Yang, Zifei ; Isenstadt, Aaron: Fuel-efficiency technology trend assessment for LDVs in China: Advanced engine technology. In: ICCT Working Paper (2018), Nr. 2018-14, S. 20. URL https://theicct.org/sites/default/files/publications/PV_Tech_ Trend_Engine_20180917.pdf. - Zugriffsdatum: 13.11.2020 
[125] Ye, Z. ; Mohamadian, H.: Model predictive control on wall wetting effect using Markov Chain Monte Carlo. In: 2013 IEEE Latin-America Conference on Communications. Santiago, Chile, 2013, S. 1-6. - DOI: https://doi.org/10.1109/LatinCom.2013.6759836. - ISSN 2330-989X

[126] Yumoto, Miki ; Goto, Kazuhiro ; Kato, Shoichi ; IIdA, Minoru: The Investigation of Mixture Formation and Combustion with Port Injection System by Visualization of Flame and Wall Film. In: SAE International Powertrains, Fuels and Lubricants Meeting. Kyoto, Japan, 2011 (SAE Technical Paper 2011-011887). - DOI: https://doi.org/10.4271/2011-01-1887. - ISSN 0148-7191

[127] Zhao, H. ; Ladommatos, N.: Optical diagnostics for soot and temperature measurement in diesel engines. In: Progress in Energy and Combustion Science 24 (1998), Nr. 3, S. 221 - 255. - DOI: http://dx.doi.org/10.1016/S03601285(97)00033-6. - ISSN 0360-1285

[128] Zhu, Rencheng ; Hu, Jingnan ; BAO, Xiaofeng ; He, Liqiang ; LAI, Yitu ; Zu, Lei ; LI, Yufei ; Su, Sheng: Tailpipe emissions from gasoline direct injection (GDI) and port fuel injection (PFI) vehicles at both low and high ambient temperatures. In: Environmental Pollution 216 (2016), S. 223 - 234. - DOI: http://dx.doi.org/10.1016/j.envpol.2016.05.066. - ISSN 0269-7491 


\section{Veröffentlichungen}

1. Schück, Claudius ; Koch, Thomas ; Samenfink, Wolfgang ; Schuenemann, Erik ; Tafel, Stephan ; Towae, Oliver: Optical Investigations of Soot Formation Mechanisms and Possible Countermeasures on a Turbocharged Port Fuel Injection SI Engine. In: SAE Int. J. Engines 9 (2016), Oct., S. 2010-2021. - DOI: https://doi.org/10.4271/2016-01-2163 - ISSN 1946-3936

2. Schück, Claudius ; Samenfink, Wolfgang ; Schünemann, Erik ; Tafel, Stephan ; Towae, Oliver ; Koch, Thomas: Analysis of particulate number emissions during dynamic load changes for port fuel injection on an optically accessible, turbocharged four-cylinder spark ignition engine. In: International Journal of Engine Research 19 (2018), Nr. 1, S. 78-85. - DOI: https://doi.org/10.1177/1468087417731044 

Logos Verlag Berlin

ISBN 978-3-8325-5258-9

ISSN 1615-2980 